\title{
Deformações Métricas e Aplicações
}

\author{
Leonardo Francisco Cavenaghi \\ TESE APRESENTADA \\ $\mathrm{AO}$ \\ Instituto De MatemáticA E EstatísticA \\ DA \\ UNIVERSIDADE DE SÃo PAUlO \\ PARA \\ OBTENÇÃO DO TÍTULO \\ $\mathrm{DE}$ \\ DOUTOR EM CIÊNCIAS
}

\author{
Programa: Doutorado em Matemática \\ Orientador: Prof. Dr. Llohann Dallagnol Sperança \\ Coorientador: Prof. Dr. Marcos Martins Alexandrino da Silva
}

Durante o desenvolvimento deste trabalho o autor recebeu auxílio financeiro da CAPES/FAPESP

São Paulo, Dezembro de 2020 
Deformações Métricas e Aplicações 
Ao Plinio véio e ao Plínio pai, que nunca entenderam, mas sempre amaram; e a Maria, por tudo. 


\section{Agradecimentos}

O autor agradece à CAPES pelo apoio financeiro no incício do doutorado e à FAPESP, processo 2017/24680-1, que foram vitais para o cumprimento desta tese.

Vida longa à ciência no Brasil! 
"Cause love's such an old fashioned word

And love dares you to care for

The people on the edge of the night

And love (people on streets) dares you to change our way of

Caring about ourselves

This is our last dance

This is our last dance

This is ourselves under pressure

Under pressure

Pressure"

David Bowie \& Queen

Começo estes versos, tais quais levam o título de "poema definitivo", com a rude certeza de que a escrita aprisiona. Assim, no sonho de ser enfim livre, me despeço da dureza de escrever, sendo que se assinarem outro texto com meu nome e caligrafia, que saibam que é falso, porque o meu espírito se vai enterrado em cada palavra, sendo o último ponto final a Mortis causa.

Porque se penso sobre viver, e o que é viver, esbarro em coisas ordinárias. Racionalizo que viver é: dançar apesar do caos, mas imediatamente deduzo que a loucura consiste em obter propósitos e sentido tão complexos (ou vazios, não sei ao certo, de fato, pois a linha que distingue o vazio do extraordinário é quase imperceptível), por meio de frases mais ou menos ditas. No que concerne a isso, sinto-me enjoado.

Me pergunto, por que viver? Sendo que por "viver", eu insisto, que seja da melhor maneira, isto é, a conduzir carreira e vida plena; se a terra é a única certeza do homem?

Torno a dizer que sim, escrever aprisiona, porque quem escreve sonha, imagina; faz de conta que ali existe um mundo todo, quando no fim, são só materializações e ideias mesquinhas que só ganham vida, e ganham sim, de fato, mas aviso, das mais passageiras, enquanto se está com o livro aberto. E tal vida tem um curto tempo de existência, aliado a um ciclo de mortes e renascimentos subsequentes, orquestrados pelos momentos em que se tiram os olhos da obra para pô-los em qualquer outra coisa.

Não entendam, entretanto, que minha vida perdeu sentido, só não sei mesmo se há sentindo, nem se faz sentido racionalizar, mas há um buraco no meu peito que não compreendo. Não, não faça alardes, não suicidarei, se não, de fato, não haveria de enfim adquirir liberdade, seria sempre "aquele tio que se matou", "o filho suicidado deles", "meu tataratio se matou", e paz nenhuma seria alcançada. Cada vez mais minha trágica história seria deturpada, a mudariam constantemente, e nasceriam livros de fofoca sobre quem fui. Não os quero. Nesse sentido, suicídio é contra o primor literário. É onde reside o auge do antiesteticismo.

Pois bem, se me quero livre, não me alongo, sigo calado, agora de versos, mas certo de que a 
viii

poesia do que se chama vida está no indescritível, na ausência de palavras, e portanto, não cabe a mim, nem a ninguém, rascunhar qualquer tentativa de exaltar o belo, quando só se consegue suprimi-lo. Tomo portanto as letras que descrevem esse devaneio e as baderno, porque ao menos no caos, $A h$ o caos, a vida encontra seu caminho.

L.F.C 


\section{Resumo}

Cavenaghi, L. F. Deformações Métricas e Aplicações. 2020. Tese (Doutorado) - Instituto de Matemática e Estatística, Universidade de São Paulo, São Paulo, 2020.

Neste trabalho estudamos diversas deformações métricas em variedades riemannianas compactas, com o intuito de encontrar condições suficientes e obstruções para existência de métricas com curvaturas seccional, de Ricci, e escalar, positivas. Precisamente:

- Entendemos a efetividade das deformações de Cheeger em promover curvatura de Ricci positiva em variedades com ações isométricas (Teorema D e Corolário E), fornecenedo uma demonstração simplificada para o Teorema A em [SW15]. Construímos exemplos (Teorema G) de variedades satisfazendo as hipóteses do Teorema A em [SW15] que não desenvolvem curvatura de Ricci positiva após qualquer deformação de Cheeger.

- Fornecemos condições suficientes para existência de métricas de curvatura de Ricci positiva e seccional quase não-negativa em algumas classes de fibrados (Teorema I), construindo exemplos de variedades com curvaturas de Ricci positiva e seccional quase não negativa (Teoremas A, B), além de exemplos de fibrados com curvatura de Ricci positiva, que incluem fibrados sobre solitons de Ricci e fibras como variedades exóticas, e com base e fibra de variedades exóticas (Capítulo 6).

- Descrevemos como realizar variedades exóticas a partir de uma contraparte clássica, e maneiras de relacionar suas geometrias, intercalando métricas de curvatura de Ricci positiva e seccional quase não-negativa entre essas.

- Apresentamos critérios para existência de métricas de curvatura seccional positiva em variedades com simetrias, análogas à condição de Chavez-Derdzinski-Rigas [CDR92] (Teorema $\mathrm{N})$.

- Estudamos o comportamento dos fluxos de curvatura média de folheações singulares riemannianas em variedades não compactas, generalizando alguns dos resultados em [AR16] (Teorema $\mathrm{O})$.

- Generalizamos o problema sobre prescrever curvatura escalar em variedades compactas, iniciado por Kazdan-Waner em [KW75a], [KW75b], [KW75c], ao contexto de fibrados topológicos (Teoremas J, K, L, e Corolário M).

Palavras-chave: variedades exóticas, fluxos de curvatura média, solitons de Ricci, geometia riemanniana, curvaturas positivas, deformações métricas, curvatura escalar prescrita. 


\section{Abstract}

Cavenaghi, L. F. Metric deformations and Applications. 2020. Tese (Doutorado) - Instituto de Matemática e Estatística, Universidade de São Paulo, São Paulo, 2020.

In this work we study several metric deformations on compact Riemannian manifolds, in order to find sufficient conditions and obstructions for the existence of metrics with positive sectional, Ricci, and scalar curvatures. Precisely:

- We understand the effectiveness of Cheeger deformations on producing metrics of positive Ricci curvature on manifolds with isometric actions (Theorem D and Corollary E), providing a simplified proof for Theorem A in [SW15]. We build examples (Theorem G) of manifolds satisfying the hypotheses in Theorem A in [SW15] that do not develope positive Ricci curvature after any Cheeger deformation.

- We provide sufficient conditions to the existence of metrics of positive Ricci curvature and almost non-negative sectional curvature on some classes of bundles (Theorem I), and build new examples of manifolds with these curvature properties (Theorems A, B), besides examples of bundles with positive Ricci curvature which includes bundles with base of Ricci solitons and fibers exotic manifolds and base and fibers exotic manifolds (Chapter 6).

- We present a general procedure to realize an exotic manifold from a classical counterpart, and ways of relate their geometries, interchanging metrics of positive Ricci curvature and almost non-negative sectional curvature between these.

- We present conditions for the existence of metrics of positive sectional curvature on manifolds with isometric actions by compact connected Lie groups, analogous to the Chavez-DerdzinskiRigas conditions [CDR92] (Theorem N).

- We study the behavior of Mean Curvature Flow of Singular Riemannian Foliations on noncompact manifolds, generalizing some of the results in [AR16] (Theorem O).

- We generalize the results on the problem of prescribing scalar curvature on compact manifolds, initially developed in [KW75a], [KW75b], [KW75c], to the setting of topological bundles (Theorems J, K, L and Corollary M).

Keywords: exotic manifolds, mean curvature flow, Ricci solitons, Riemannian geometry, positive curvatures, metric deformations, prescribed scalar curvature. 


\section{Sumário}

1 Introdução 1

2 Introduction $\quad 9$

3 Algumas deformações métricas e aplicações $\quad 17$

3.1 Folheações métricas . . . . . . . . . . . . . . . . . . . . . . . . . . . . . 17

3.1 .1 Submersões riemannianas e fibrados topológicos . . . . . . . . . . . . . 17

3.1.2 Folheações riemannianas singulares e ações isométricas . . . . . . . . . . . . . 26

3.2 Deformações métricas clássicas . . . . . . . . . . . . . . . . . . 30

3.3 Um mínimo sobre fluxos geométricos: Solitons de Ricci e Fluxos de Curvatura Média 34

3.3.1 Mapas harmônicos e fluxos de curvatura média . . . . . . . . . . . . . . 35

3.3.2 Fluxos de Ricci e Solitons de Ricci . . . . . . . . . . . . . . . . . . . . . . . 41

4 Diagramas Estrela: topologia e geometria $\quad 45$

4.1 Construindo diagramas estrela . . . . . . . . . . . . . . . . . 45

4.2 A geometria dos diagramas estrela . . . . . . . . . . . . . . . . 5 50

4.3 Realizações explícitas de variedades exóticas via diagramas estrela (um esboço) . . . 51

5 Deformações de Cheeger $\quad 55$

5.1 Deformações de Cheeger clássicas . . . . . . . . . . . . . . . . . 55

5.2 Sobre a efetividade das deformações de Cheeger clássicas . . . . . . . . . . . . . . 61

$5.2 .1 \quad$ A geometria em pontos singulares . . . . . . . . . . . . . . . . . . 62

5.2.2 Eixos fixos e uma descrição algébrica da curvatura de Ricci . . . . . . . . . . 66

5.2.3 Uma descrição algébrica do tensor de Ricci em eixos fixos . . . . . . . . . . . 68

5.2 .4 Uma família de contra-exemplos . . . . . . . . . . . . . . . . 71

5.2.5 Um critério algébrico para não positividade da curvatura de Ricci . . . . . . . 72

5.3 Um refinamento para o Teorema de Lawson-Yau . . . . . . . . . . . . . . . . 73

5.4 Uma demonstração simplificada do Teorema de Searle-Wilhelm . . . . . . . . . . . . 74

5.5 Deformações de Cheeger aplicadas à diagramas estrela . . . . . . . . . . . . 76

5.6 O conceito de deformações de Cheeger em fibrados com grupo de estrutura compacto 79

5.7 Uma condição análoga à de Chavez-Derdzinski-Rigas . . . . . . . . . . . . . . . . 85

$\begin{array}{llr}6 & \text { Um zoológico de variedades } & 89\end{array}$

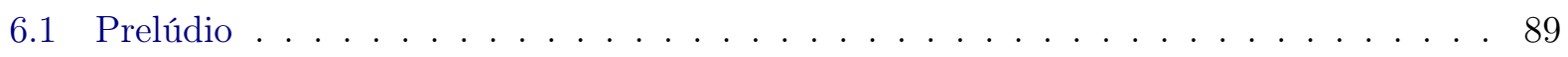

6.2 Construção dos exemplos . . . . . . . . . . . . . . . . . . . . . 90 
7 Sobre funções de curvatura escalar admssíveis em fibrados com grupo de estrutura compacto

7.1 Fibrados Topológicos com fibras com curvatura escalar positiva . . . . . . . . . . . 95

7.2 Fibrados Topológicos com fibras com curvatura escalar com sinal misto . . . . . . . . 97

A Rudimentos de análise $\quad 107$

A.1 Análise básica em variedades . . . . . . . . . . . . . . . . . . . . . 107

A.1.1 Espaços de Lebesgue e Sobolev . . . . . . . . . . . . . . . . . . . . . . 107

A.2 Kondrakov e Poincaré: inclusões e desigualdades . . . . . . . . . . . . . . . . . . . 108

A.3 Operadores Diferenciais, equações elípticas e problemas variacionais . . . . . . . . . . 108

A.3.1 Definições gerais . . . . . . . . . . . . . . . . . . . . 108

A.3.2 Operadores Elípticos e equações diferenciais parciais elípticas . . . . . . . . . 109

A.4 Um mínimo sobre fluxos gradientes e funcionais de energia . . . . . . . . . . . . . . . 110

Referências Bibliográficas 


\section{Capítulo 1}

\section{Introdução}

Um fato bem conhecido em geometria é a sua própria utilidade na compreensão de variedades como espaços topológicos. Em verdade, resultados como o Teorema da Esfera Diferenciável (veja [BS]), e o programa de geometrização de Thurston (veja [Thu82, Per03]), classificam a topologia de variedades riemannianas de acordo com sua geometria.

Por outro lado, o problema recíproco encontra-se não resolvido para quase toda variedade, sendo pouco conhecidas as possiveis propriedades geométricas que uma variedade fixada pode assumir. Pode-se, portanto, recorrer ao entendimento de uma família rica de exemplos com propriedades determinadas para entender a relação entre topologia e geometria. Tal abordagem mostra-se frutífera, por exemplo, na construção da Esfera de Berger e, mais geralmente, no estudo da geometria de todas as métricas de uma determinada variedade, tratamento esse extensivamente utilizado no caso homogêneo (veja [MG09, ST09, Sta16]).

Embora existam teoremas como o de Preissman no contexto de variedades riemannianas com curvatura seccional negativa e de Bonnet-Meyers sobre variedades com curvatura de Ricci positiva, não há teoremas que distinguam quais elementos na classe de variedades compactas simplesmente conexas admitem uma métrica de curvatura não negativa mas não uma de curvatura positiva. Segundo esse fato, poderia-se acreditar que toda variedade simplesmente conexa de curvatura não negativa admite métrica de curvatura positiva. Nota-se, porém, que a literatura apresenta uma diferença gritante entre a diversidade de exemplos entre esses dois caos (veja por exemplo [Zil14], [Zil07]). Existem variedades suaves $\Sigma^{n}$ que são homeomorfas às esferas padrão $S^{n}$, mas não são difeomorfas a essas. Também existem incontáveis estruturas suaves (não difeomorfas par a par) em $\mathbb{R}^{4}$, assim como existem toros exóticos, espaços projetivos exóticos, e assim por diante. Naturalmente surge a pergunta: quanto a estrutura diferenciável determina a geometria? Mais concretamente: essas esferas exóticas admitem geometrias semelhantes às de $S^{n}$ ?

Essa questão tem sido constantemente explorada. Por um lado, Gromoll-Meyer construíram a primeira esfera exótica com uma métrica de curvatura seccional não negativa (veja [GM74]); Wilhelm construiu métricas com curvatura seccional quase não negativa (com curvatura de Ricci positiva) em cada esfera exótica de dimensão 7 (veja [Wil01]); posteriormente, Grove-Ziller e GoetteKerin-Shankar construíram métricas de curvatura seccional não negativa nesses exemplos (veja [GZ00], [GKS20]). Grove-Verdiani-Wilking-Ziller mostraram que algumas esferas exóticas não suportam métricas com curvatura seccional não negativa e com muitas simetrias (veja [GVWZ06]); e Grove-Verdiani-Ziller construíram um espaço tangente unitário exótico (ver [GVZ11]) com curvatura seccional positiva. Em relação à existência de métricas com curvatura de Ricci positiva, Nash ([Nas79]), Poor ([Poo75]), Searle-Wilhelm ([SW15]), Wraith, Joachim and Crowley ([Wra97, Wra07], [JW08] and [CN15, CW17a, CW17b]) mostraram a existência de métricas de curvatura positiva de Ricci em alguns variedades exóticas. Em [CS18b, CS19], Prof. Sperança e eu construímos métricas de curvatura de Ricci positiva em diversas variedades exóticas e fibrados com fibras e/ou bases de variedades exóticas. Por outro lado, não se sabe se existe uma esfera exótica com curvatura seccional positiva, e Hitchin provou que existem esferas exóticas que nem mesmo admitem métricas de curvatura escalar positiva (veja [Hit74]). Dessa maneira, encontrar obstruções/fornecer condições 
suficientes, e sempre que possível, construir exemplos de variedades com alguma curvatura positiva, podem fornecer uma nova perspectiva para essa área.

Dito isso, esta tese tem como objetivos principais, entender condições necessárias e suficientes para existência de métricas com curvaturas positivas (seccional, Ricci, escalar) em variedades com alguma simetria, e fornecer construções explícitas de diversos novos exemplos de variedades (incluindo exóticas) com curvaturas seccionais quase não negativa e Ricci positiva (veja a seção 5.5 e o Capítulo 6).

Uma ferramenta comum na construção de exemplos de métricas com curvaturas não negativa/positiva é o processo conhecido como deformações de Cheeger (veja, e.g., Cheeger [Che73, Zil, M̈̈8]), que se aplica a $G$-variedades, ou seja, variedades riemannianas munidas de uma ação isométricas efetiva por um grupo de Lie compacto e conexo $G$. Na presente tese, para obter a maior parte dos resultados fazemos uso direta, ou indiretamente, dessas deformações, introduzidas no Capítulo 5.

A primeira parte deste trabalho consiste em sumarizar um procedimento padrão, iniciado por Durán em [Dur01], que objetiva realizar a construção topológica de variedades exóticas via uma contraparte clássica. A grande vantagem desse método reside na possibilidade de traduzir algumas proriedades geométricas de objetos clássicos para objetos exóticos. Precisamente, considera-se diagramas como:

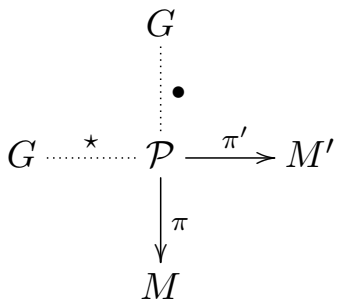

onde as submersões $\pi$ e $\pi^{\prime}$ definem fibrados principais e as ações $\bullet, \star$ comutam em $\mathcal{P}$.

Apresentaremos sucintamente no Capítulo 4 uma abordagem sistemática para construção desse tipo de diagrama, extensivamente explorada em [Dur01, DMR04, Spe10, Spe16a]. A ideia consiste em tomar uma tripla bem conhecida $(G, \mathcal{P}, M)$ para realizar variedades exóticas (aqui representadas por $M^{\prime}$ ). As informações topológias sobre a construção desse tipo de fibrado são decisivas para reconhecer $M^{\prime}$ como o candidato exótico que se espera realizar.

A apresentação desenvolvida no Capítulo 4 é embasada no meu primeiro artigo publicado, [CS18c]. No entanto, vale ressaltar que no que concerne à realizações topológicas, a contribuição foi dada majoritariamente pelo orientador, Prof. Sperança. Assim sendo, discutiremos rapidamente as técnicas básicas, mas focando majoritamente na parte geométrica desse tipo de construção, uma vez que essa é de fato a contribuição do candidato ao tema. Em particular, faremos uso ds deformações de Cheeger para provar existência de novas métricas de curvatura seccional quase não negativa e com curvatura de Ricci positiva em diversas variedades obtidas dessa maneira (veja a seção 5.5). A saber, destacamos como primeiro teorema original:

Teorema A. Sejam $\Sigma^{7}$ e $\Sigma^{8}$ quaisquer variedades fechadas homotópicas à esferas padrões de dimensões 7 e 8 , respectivamente; $\Sigma^{10}$ qualquer variedade fechada homotópica à 10-esfera e que seja bordo de uma variedade spin; $\Sigma^{4 m+1}, \Sigma^{8 m+5}$ esfera de Kervaire em dimensões $4 m+1,8 m+5$, respectivamente. Então cada variedade abaixo admite uma sequência de métricas $\left\{g_{n}\right\}_{n \in \mathbb{N}}$ com curvatura de Ricci positiva satisfazendo $\sec _{g_{n}} \geq-1 / n$ e $\operatorname{diam}_{g_{n}} \leq 1 / n$ :

(i) $M^{7} \# \Sigma^{7}$, onde $M^{7}$ é qualquer $S^{3}$-fibrado sobre $S^{4}$

(ii) $M^{8} \# \Sigma^{8}$, onde $M^{8}$ é ou um $S^{3}$-fibrado sobre $S^{5}$ ou um $S^{4}$-fibrado sobre $S^{4}$

(iii) $M^{10} \# \Sigma^{10}$, onde:

(a) $M^{10}=M^{8} \times S^{2}$ com $M^{8}$ como no item (ii)

(b) $M^{10}$ é qualquer $S^{3}$-fibrado sobre $S^{7}, S^{5}$-fibrado sobre $S^{5}$ ou $S^{6}$-fibrado sobre $S^{4}$

(iv) $M^{4 m+1} \# \Sigma^{4 m+1}$ onde 
(a) $S^{2 m} \hookrightarrow M^{4 m+1} \rightarrow S^{2 m+1}$ é o fibrado de esferas associado a qualquer múltiplo de ${ }^{1}$ de $O(2 m+1) \hookrightarrow O(2 m+2) \rightarrow S^{2 m+1}$, fibrado de referenciais de $S^{2 m+1}$

(b) $\mathbb{C} P^{m} \hookrightarrow M^{4 m+1} \rightarrow S^{2 m+1}$ é o $\mathbb{C} P^{m}$-fibrado associado a qualquer múltiplo ${ }^{1}$ do fibrado de referências unitários $U(m) \hookrightarrow U(m+1) \rightarrow S^{2 m+1}$

(c) $M^{4 m+1}=\frac{U(m+2)}{S U(2) \times U(m)}$

(v) $\left(M^{8 r+k} \times N^{5-k}\right) \# \Sigma^{8 r+5}$ onde $N^{5-k}$ é qualquer variedade com curvatura de Ricci positiva

(a) $S^{4 r+k-1} \hookrightarrow M^{8 r+k} \rightarrow S^{4 r+1}$ é a $k$-ésima suspensão do fibrado tangente unitário $S^{4 r-1} \hookrightarrow$ $T^{1} S^{4 r+1} \rightarrow S^{4 r+1}$,

(b) para $k=1, \mathbb{H} P^{m} \hookrightarrow M^{8 m+1} \rightarrow S^{4 m+1}$ é o $\mathbb{H} P^{m}$-fibrado associado a qualquer múltiplo ${ }^{1}$ de $S p(m) \hookrightarrow S p(m+1) \rightarrow S^{4 m+1}$

(c) para $k=0, M=\frac{S p(m+2)}{S p(2) \times S p(m)}$

(d) para $k=1, M=M^{8 m+1}$ é como no item (iv).

As mesmas técnicas permitem provar que:

Teorema B. Os seguintes espaços projetivos exóticos $\Sigma \mathcal{P} \mathbb{C}^{n-1}, \Sigma \mathcal{P} \mathbb{H}^{m-1}$ possuem métricas $U(n / 2)$, $S p(m / 2)$-invariantes, respectivamente, com curvatura de Ricci positiva, onde

$$
\begin{aligned}
\Sigma \mathcal{P} \mathbb{C}^{n-1} & :=\Sigma^{2 n-1} / S^{1}, \\
\Sigma \mathcal{P} \mathbb{H}^{m-1} & :=\Sigma^{4 m-1} / S U(2) .
\end{aligned}
$$

Talvez a principal razão para que na construção de variedades com alguma propriedade de curvatura positiva, os exemplos sejam obtidos via submersões, se deva à fórmula de submersão de Gray-O'Neill, visto que essa implica que a curvatura seccional da base é maior ou igual que a curvatura seccional do espaço total (em direções horizontais). No entanto, não é necessariamente verdade que submersões riemannianas preservam curvatura de Ricci positiva (veja [PW14]).

A pergunta recíproca, a saber: dada uma submersão riemanniana com base de curvatura seccional positiva, é possivel obter uma métrica (de submersão) com curvatura seccional positiva no espaço total? admite um fácil contra-exemplo. Basta considerar como espaço total a variedade $\mathbb{R} P^{2} \times \mathbb{R} P^{2}$ munida da ação transitiva de $S O(3)$ no segundo fator. A base dessa submersão (que é o espaço das órbitas dessa ação) é $\mathbb{R} P^{2}$, que tem curvatura seccional positiva na métrica de submersão $S^{2} \rightarrow \mathbb{R} P^{2}$. Entretanto, pelo Teorema de Synge, a curvatura positiva não pode ser levantada para o espaço total pois $\mathbb{R} P^{2} \times \mathbb{R} P^{2}$ não é simplesmente conexo.

A seguinte pergunta natural seria portanto: o mesmo vale para curvaturas de Ricci e escalar? Searle-Wilhelm em [SW15] mostraram que para uma grande classe de exemplos, é possível levantar curvatura de Ricci positiva do espaço de órbitas associado a uma variedade com ação isométrica por um grupo de Lie compacto.

Teorema C (Searle-Wilhlem). Seja $(M, g)$ uma G-variedade riemanniana onde $M$ e $G$ são compactos. Assuma que

1. Uma órbita principal de $G$ em $M$ possui grupo fundamental finito,

2. $\operatorname{Ric}_{M / G} \geq 1$ na métrica de distância orbital.

Então $M$ admite uma métrica $G$-invariante com curvatura de Ricci positiva.

A ideia princial para a prova do Teorema $\mathrm{C}$ consiste em realizar uma transformação conforme da métrica $g$, seguida de uma deformação de Cheeger. A demonstração desse é bastante complicada e dividida em várias etapas, de modo que deu origem às seguintes perguntas:

\footnotetext{
${ }^{1} \mathrm{Ou}$ seja, um fibrado cujas funções de transição $\alpha: S^{n-1} \rightarrow G$ são pontualmente múltiplas de $\tau_{2 m}: S^{2 m} \rightarrow$ $O(2 m+1), \tau_{m}^{\mathbb{C}}: S^{2 m} \rightarrow U(m)$ or $\tau_{m}^{\mathbb{H}}: S^{4 m+2} \rightarrow S p(m)$, funções de transição do fibrado de referenciais ortonormais e suas reduções, respectivamente.
} 
É possivel provar esse resultado utilizando apenas deformações de Cheeger? Isso simplificaria a demonstração?

$\mathrm{Na}$ presente tese, as respondemos completamente. Para tanto, estudamos o comportamento dos tensores associados às deformações de Cheeger em pontos singulares, isto é, pontos onde a dimensão do espaço horizontal é maior (isto é, a dimensão do espaço complementar à órbita) (em consequência da isotropia maior). Esse acréscimo de direções horizontais é o que dificulta o controle da curvatura de Ricci, uma vez que os termos novos poderiam contribuir com sinal negativo. Por outro lado, afim de conseguir uma resposta afirmativa, era esperado um comportamento de blow-up de quantidades positivas na expressão da curvatura de Ricci. Observamos que esse blow-up de fato ocorre (Corolário E e Teorema H) mas em certas direções (Teoremas D, F e G). Precisamente:

Teorema D. Seja $\left(M^{n}, g\right)$ uma variedade riemanniana compacta e conexa munida de uma ação isométrica por um grupo de Lie compacto e conexo $G$. Denote por $\mathcal{H}_{p}$ o espaço horizontal da $G$-ação em p. Assuma que:

1. Uma órbita principal de $G$ em $M$ tenha grupo fundamental finito,

2. $\operatorname{Ric}_{M^{\text {reg }} / G} \geq 1$

Se g tem direções de curvatura de Ricci negativa para cada deformação de Cheeger finita, então

(a) Existe um ponto singular $p \in M$ com um vetor não nulo $X \in \mathcal{H}_{p}$ fixado pela representação de isotropia $\rho: G_{p} \rightarrow O\left(\mathcal{H}_{p}\right)$,

(b) A restrição de $\rho$ a $X^{\perp} \cap \mathcal{H}_{p}$ é redutivel.

Ainda mais, se $X^{\perp} \cap \mathcal{H}_{p}$ tem exatamente dois somandos $\rho$-irredutiveis, $X^{\perp} \cap \mathcal{H}_{p}=\mathcal{H}_{1}+\mathcal{H}_{2}$, então (a menos de alterar a ordem dos somandos), para qualquer vetor regular $Y=Y_{1}+Y_{2} \in \mathcal{H}_{1}+\mathcal{H}_{2}$,

$$
\operatorname{dim} \mathcal{H}_{1}-\operatorname{dim} G_{p} Y_{1}>\frac{(k-1) \operatorname{dim} \mathcal{H}_{1}}{\operatorname{dim} \mathcal{H}_{p}-1},
$$

onde $k$ é a codimensão de uma órbita principal em $M$.

Em particular, nesse caso, onde as deformações de Cheeger são inefetivas, existe uma geodésica em $M$ totalmente contida no estrato singular. Como consequência natural, temos:

Corolário E. Seja $(M, g)$ uma variedade riemanniana compacta e conexa munida de uma métrica $G$-invariante, onde $G$ é um grupo de Lie compacto e conexo. Assuma que:

1. Uma órbita principal $G$ em $M$ tenha grupo fundamental finito,

2. $\operatorname{Ric}_{M^{r e g} / G} \geq 1$,

Então, g desenvolve uma métrica de curvatura de Ricci positiva após deformação de Cheeger finita se uma das condições equivalentes é satisfeita:

(a) O estrato singular é composto de órbitas isoladas;

(b) Para todo ponto singular p, a representação linear de isotropia é irredutivel;

(c) Nenhuma representação de isotropia tem um vetor não nulo fixo;

(d) A ação induzida de $G$ no espaço tangente unitário $T_{1} M$ não tem pontos fixos.

Uma recíproca local para o Teorema D também foi obtida:

Teorema F. Seja $G$ um grupo de Lie compacto agindo em uma variedade compacta e conexa $M$. Assuma que: 
(a') Existe um ponto fixo $p$ cuja representação de isotropia, $\rho: G_{p} \rightarrow O\left(\mathcal{H}_{p}\right)$, fixa um vetor não nulo $X \in \mathcal{H}_{p}$,

(b') $X^{\perp} \cap \mathcal{H}_{p}$ se decompõe em dois subespaços $\rho$-invariantes, $X^{\perp} \cap \mathcal{H}_{p}=\mathcal{H}_{1}+\mathcal{H}_{2}$, satisfazendo

$$
\operatorname{dim} \mathcal{H}_{1}-\operatorname{dim} G_{p} Y_{1}>\frac{(k-1) \operatorname{dim} \mathcal{H}_{1}}{\operatorname{dim} \mathcal{H}_{p}-1}
$$

para todo vetor regular $Y \in X^{\perp} \cap \mathcal{H}_{p}$.

Então existe uma vizinhança $G$-invariante de $p$ e uma métrica $G$-invariante $g$ satisfazendo

1. $\operatorname{Ric}_{M^{\text {reg }} / G} \geq 1$

2. $\operatorname{Ric}_{g_{t}}(X)<0$ para toda deformação de Cheeger $g_{t}$ de $g$

Vale notar que o Teorema $\mathrm{F}$ não assume que $\mathcal{H}_{1}, \mathcal{H}_{2}$ sejam os únicos subespaços invariantes não triviais de $\left.\rho\right|_{X^{\perp} \cap \mathcal{H}_{p}}$.

A natureza algébrica de nossos resultados segue do fato que: de [SW15, Proposition 3.1], sabemos que vetores com curvatura de Ricci negativa só podem ocorrer em pontos singulares. Mais ainda, as estimativas apresentadas na seção 5.2 garantem que tais vetores são fixos pela representação de isotropia em seu ponto base. Então, dado tal vetor $X \in \mathcal{H}_{p}$, o Lema de Schur garante que a restrição de $R_{X}(Y)=R(Y, X) X$ a cada subespaço $\rho$-irredutível é um múltiplo da identidade. As desigualdades em D e F seguem de argumentos combinatoriais (veja, por exemplo as subseções 5.2.3 e 5.2.5).

Como consequência de todas as técnicas desenvolvidas, foi possível construir uma família de exemplos globais onde as deformações de Cheeger são inefetivas.

Teorema G. Seja $S^{n}$ a esfera unitária $\mathbb{R}^{n+1}$ munida da ação padrão de $S O(n-2)$ fixando as 3 primeiras coordenadas. Então, para cada $n \geq 5$, existe uma métrica $S O(n-2)$-invariante $g$ em $S^{n}$ satisfazendo:

1. $\operatorname{Ric}_{M^{r e g} / G} \geq 1$

2. Existe um vetor não nulo $X \in \mathcal{H}_{p}$ tal que $\operatorname{Ric}_{g_{t}}(X)<0$ para toda deformação de Cheeger $g_{t}$.

Ademais, as estimativas dos pontos singulares permite apresentar uma demonstração bem mais simples para o clássico resultado em [LS74] com uma pequena melhora.

Teorema H. Seja $(M, g)$ uma variedade riemanniana compacta munida de uma ação de um grupo de Lie compacto, conexo e não abeliano $G$. Então $g$ desenvolve curvatura escalar positiva após deformação de Cheeger finita.

Por fim, re-demonstramos (de maneira bem mais simples) o Teorema C (Teorema 5.4.1).

$\mathrm{Na}$ subseção 5.6, estendemos o conceito de deformação de Cheeger ao contexto de fibrados com grupo estrutural compacto. Se $F \hookrightarrow M \stackrel{\pi}{\rightarrow} B$ é um fibrado suave com grupo de estrutura $G$, seja $\mathcal{P}$ o fibrado principal associado a $\pi$. Lembre que existe uma submersão riemanniana $\bar{\pi}$ : $\left(\mathcal{P} \times F, g_{\mathcal{P}} \times g_{F}\right) \rightarrow(M, h)$, onde $g$ e $g_{F}$ são métricas $G$-invariantes em $\mathcal{P}$ e $F$, respectivamente.

A ideia consiste em realizar uma deformação de Cheeger na $G \times G$-variedade $\mathcal{P} \times F$. A partir da submersão $\bar{\pi}$ consegue-se uma família de métricas riemannianas $h_{t}$ em $M$. Provamos que:

Teorema I. Seja $F \hookrightarrow M \stackrel{\pi}{\rightarrow} B$ um fibrado topológico com base $B$, fibra $F$ e grupo estrutural $G$ compactos e conexos. Assuma que:

1. As órbitas de $G$ em $\mathcal{P}$ tenham curvatura seccional positiva em uma métrica bi-invariante;

2. Exista uma sequência de métricas $g_{n}$ em $\mathcal{P}$ com curvatura de Ricci positiva e tais que $K_{g_{n}}\left(X^{\mathcal{H}}, Y\right) \geq$ $-\frac{1}{n}, \forall X, Y \in T P, e \operatorname{diam}\left(g_{n}\right) \leq \frac{1}{n} ;$ 
3. $g_{F}$ possua uma métrica $G$-invariante de curvatura seccional não negativa e Ricci positiva;

Então $M$ admite uma sequência de métricas $\widetilde{g}_{n}$ com curvatura de Ricci positiva e tais que diam $\left(\widetilde{g}_{n}\right) \leq$ $\frac{1}{n}, \sec \left(\widetilde{g}_{n}\right) \geq-\frac{1}{n}$.

Como contribuições finais, estudamos o problema de prescrever métricas de curvatura escalar em certas submersões riemannianas, sendo uma extensão natural do clássico problema de KazdanWarner (veja [KW75b], [KW75a], [KW75c]). Provamos que:

Teorema J. Seja $F \hookrightarrow M \stackrel{\pi}{\rightarrow} B$ um fibrado com $M, F, B$ e grupo estrutural $G$ compactos. Assuma que:

1. Uma órbita principal de $G$ em $F$ tem grupo fundamental finito,

2. F possua uma métrica $G$-invariante tal que $\operatorname{Ric}_{F^{r e g} / G} \geq 1$.

\section{Então}

1. Existe $\lambda \in(0,1]$, que depende só da geometria da fibra, tal que qualquer função suave $f: M \rightarrow$ $\mathbb{R}$ satisfazendo $\frac{\min _{p \in B} f}{\max _{p \in B} f} \leq \lambda$ é curvatura escalar de uma métrica de submersão completa em $M$, exceto possitivelmente se $f=$ cte $\geq 0$;

2. Se $F$ possui curvatura escalar constante, então toda função suave $f: M \rightarrow \mathbb{R}$ é curvatura escalar de uma métrica de submersão completa $M$, exceto possivelmente se $f=$ cte $\geq 0$.

No caso em que a fibra possui pontos com curvatura escalar não-positiva, seguimos uma abordagem via métodos variacionais, obtendo os seguintes resultados. Defina as constantes

$$
b_{k}:=\frac{k+1}{8 k}, c_{k}:=\frac{(k+1)^{2}}{8(k-1) k}, \theta:=\frac{2(k-1)}{k+1} .
$$

Teorema K. Seja $\pi:\left(F^{k}, g_{F}\right) \hookrightarrow\left(M^{n+k}, g\right) \rightarrow\left(B^{n}, h\right)$ uma submersão riemanniana com $M$ fechada e conexa e $F$ compacta e conexa. Assuma que:

1. $\min _{F} \operatorname{scal}_{F} \leq 0$,

2. $\left(F, g_{F}\right)$ seja subvariedade minima de $(M, g)$,

3. Se A denota o tensor de Gray-O'Neill da submersão $\pi$, então

$$
\max _{M}\left\{3 \sum_{i, j}\left|A_{e_{i}} e_{j}\right|^{2}-2 \sum_{i, r}\left|A_{e_{i}}^{*} v_{r}\right|^{2}\right\} \leq 0
$$

onde $\left\{e_{i}\right\}$ é uma base $g$-ortonormal para $\mathcal{H}$ e $\left\{v_{i}\right\}$ é uma base g-ortonormal para $\mathcal{V}$.

Então qualquer função $f \in C^{\infty}(M ; \mathbb{R})$ satisfazendo

$$
\frac{\lambda_{1}}{2+\epsilon}-b_{k} \max _{M}\left(f-\mathrm{scal}_{g}+\operatorname{scal}_{F}\right)+c_{k} \min _{F} \operatorname{scal}_{F}(\operatorname{vol}(B))^{2 / \theta-1}+b_{k} \min _{M} \delta A>0,
$$

para algum $\epsilon>0$, onde $\lambda_{1}$ denota o primeiro autovalor positivo de $-\Delta_{B}$, é função de curvatura escalar para alguma métrica de submersão em $M$.

No caso em que $M$ é isométrica a um produto, uma vez que as fibras são totalmente geodésicas e $A \equiv 0$, obtemos: 
Teorema L. Seja $M=\left(B^{n} \times F^{k}, g_{B} \times g_{F}\right)$ uma variedade riemanniana fechada e conexa. Suponha que $\min _{F} \operatorname{scal}_{F} \leq 0$. Então qualquer função suave $f \in C^{\infty}(M ; \mathbb{R})$ satisfazendo

$$
\frac{\lambda_{1}}{2+\epsilon}-b_{k} \max _{B}\left(f-\operatorname{scal}_{B}\right)+c_{k} \min _{F} \operatorname{scal}_{F}(\operatorname{vol}(B))^{2 / \theta-1}>0,
$$

para algum $\epsilon>0$, onde $\lambda_{1}$ denota o primeiro autovalor positivo de $-\Delta_{B}$, é função de curvatura escalar para alguma métrica de submersão em $M$.

Os Teoremas 7.2.1 e 7.2.2 são uma generalização natural do problema de preescrever curvatura escalar constante via produtos verticais, amplamente discutidos em [DD87], [EYTK96]. Sob a hipótese curvatura de Ricci positiva em $B$ e $M$ isométrica a um produto, conseguimos outra condição.

Teorema M. Seja $M^{n+k}=\left(B^{n} \times F^{k}, g_{B} \times g_{F}\right)$. Assuma que $\operatorname{Ric}\left(g_{B}\right) \geq(n-1)$ e que $\min _{F} \operatorname{scal}_{F} \leq 0$. Então qualquer função suave $f: M \rightarrow \mathbb{R}$ tal que

$$
n\left(\frac{8 k}{(2+\epsilon)(k+1)}+(n-1)\right)>\max _{B} f+\frac{c_{k}}{b_{k}} \min _{F} \operatorname{scal}_{F}(\operatorname{vol}(B))^{2 / \theta-1},
$$

para algum $\epsilon>0$, é função de curvatura escalar para alguma métrica de submersão em $M$.

Por fim, obtivemos uma condição necessária e suficiente para que uma $G$-variedade admita uma métrica de curvatura seccional positiva (Teorema N); e em colaboração com o co-orientador Prof. Alexandrino, obtivemos alguns resultados referentes a fluxos de curvatura média em folheações singulares riemannianas (Teorema $\mathrm{O}$ ):

Teorema N. Seja $(M, g)$ uma variedade riemanniana compacta munida de uma ação isométrica por um grupo de Lie compacto e conexo $G$. Fixe uma métrica bi-invariante $Q$ em $G$. Assuma que para cada $p \in M^{r e g}, G / G_{p}$ possua curvatura seccional positiva na métrica de espaço homogêneo normal. Então existe $t>0$ tal que a deformação de Cheeger $g_{t}$ de $g$ satisfaz $\sec _{g_{t}}>0$ se, e somente se, existir $k>0$ tal que

$$
\begin{aligned}
\left(K_{B}(X, Y)-k|X \wedge Y|_{g}^{2}\right)\left(\frac{1}{2} Q\left(\operatorname{Hess} P^{-1}(X) V, V\right)+\frac{1}{4}\left|\Omega_{X}^{*} V\right|_{g}^{2}-k|X|_{g}^{2} Q\left(P^{-1} V, V\right)\right) \\
\geq \frac{1}{4} Q\left(\left(\nabla_{X} \Omega\right)_{X} Y, V\right)^{2}
\end{aligned}
$$

para todos $X, Y \in \mathcal{H}_{p}, V \in \mathfrak{m}_{p}, p \in M^{\text {reg }}$, onde $\mathfrak{m}_{p}$ é identificado com o espaço tangente à órbita de $G$ por $p$; $P$ é o único tensor simétrico positivo definido tal que $g(V, W)=Q(P V, W)$ e $\Omega$ é a 2-forma de curvatura do fibrado principal

$$
M^{r e g} \rightarrow M^{r e g} / G
$$

Teorema O. Sejam $(M, g)$ uma variedade riemanniana completa munida de uma folheação singular riemanniana com folhas fechadas $\mathcal{F}$. Assuma que

1. $M$ tenha curvatura seccional limitada;

2. O operador formato ao longo de cada folha $L \in \mathcal{F}$ seja limitado;

3. O quociente $M / \mathcal{F}$ seja compacto.

Seja $N$ uma subvariedade imersa contida em uma folha regular. Assuma que a dimensão de $N$ seja maior que a dimensão das folhas singulares, e que o fluxo de curvatura média $N(t)$ seja a restrição de um fluxo básico (com respeito a $\mathcal{F}$ ) no estrato regular, cujo intervalo máximo de existência é $[0, T), T<\infty$. Então $N(t)$ converge a uma folha singular no espaço das folhas. 


\section{Capítulo 2}

\section{Introduction}

A well known fact in geometry consists of its own usage on the understanding of manifolds as topological spaces. For instance, Theorems like the Differentiable Sphere Theorem (see [BS]), and the Thurston's Geometrization Program (see [Thu82, Per03]), classify the topology of some Riemannian manifolds according to their geometries.

On the other hand, the converse problem remains unsolved for almost any manifold, being few the geometric properties known that a given manifold can assume. Therefore, one could consider rich families of manifolds with predetermined properties to try to understand the relation between topology and geometry. Such approach has been fruitful in the homogeneous setting, such as the constructions of Berger's spheres and, more generally, in the study of all Riemannian metrics on a fixed class of homogeneous space (see [MG09, ST09, Sta16]).

Although there are results like Preissman's Theorem in the setting of Riemannian manifolds with negative sectional curvature, and the Bonnet-Meyers theorem for manifolds with positive Ricci curvature bounded from below, there is no theorem that distinguishes the class of closed simply connected manifolds with non-negative sectional curvature to the one of closed simply connected manifolds admitting metrics with positive sectional curvature. According to this fact it would be natural to expect that every manifold on the first mentioned class admits a metric of positive sectional curvature. However, the literature presents a huge discrepancy between the examples on these classes (compare [Zil14] to [Zil07]). There exist smooth manifolds $\Sigma^{n}$ that are homeomorphic to the standard sphere $S^{n}$, but not diffeomorphic to it. There are also uncountably many smooth structures (pairwise not diffeomorphic) on $\mathbb{R}^{4}$, as well as there are exotic tori, exotic projective spaces, and so on. It naturally raises the question how much the smooth structure determines the geometry? More specifically, such exotic spheres admit similar geometries to the standard geometries on $S^{n}$ ?

These questions have been constantly explored. On one hand, Gromoll-Meyer constructed the first exotic sphere with a metric of non-negative sectional curvature (see [GM74]); Wilhelm constructed metric of positive Ricci curvature and almost non-negative sectional curvature in every exotic sphere of dimension 7 (see [Wil01]); later Grove-Ziller and Goette-Kerin-Shankar built metrics of non-negative sectional curvature on these examples (see [GZ00], [GKS20]). Grove-VerdianiWilking-Ziller showed that some exotic spheres do not support metrics of non-negative sectional curvature with lots of symmetries (see [GVWZ06]); and Grove-Verdiani-Ziller build an exotic unitary tangent space (see [GVZ11]) with positive sectional curvature. Concerning manifolds with metrics of positive Ricci curvature, Nash ([Nas79]), Poor ([Poo75]), Searle-Wilhelm ([SW15]), Wraith, Joachim and Crowley ([Wra97, Wra07], [JW08] and [CN15, CW17a, CW17b]) proved the existence of these metrics on some exotic manifolds. In [CS18b, CS19], Prof. Sperança and I built metrics of positive Ricci curvature on several exotic manifolds and bundles with fibers and/or bases of exotic manifolds. On the other hand, it is now known is there exists an exotic sphere with a metric of positive sectional curvature, and Hitchin proved that there are exotic spheres that do not even admit metrics of positive scalar curvature (see [Hit74]). Therefore, to find obstructions/providing sufficient conditions and possibly construct examples of manifolds with some positive curvature 
property may lead to a new perspective in this area.

That said, this thesis has as main goals, to understand necessary and sufficient conditions to the existence of metrics with positive sectional, Ricci and scalar curvatures on manifolds with some symmetry assumption, and provide explicit constructions of several new examples of manifolds (including exotic ones) with some of these curvature properties (see the section 5.5 and the Chapter $6)$.

A standard tool on the construction of manifolds with non-negative/positive sectional curvature is the technique known as Cheeger deformations (see for instance [Che73, Zil, M8̈7]), applied to Gmanifolds, i.e, manifolds with isometric actions by compact connected Lie groups. In this thesis most of the obtained results are by the means of such deformations, introduced in the Chapter 5 .

The first part of this work consists in summarizing a standard procedure, started by Durán in [Dur01], to realize exotic manifolds via a counterpart classical manifold. The great advantage coming from this relies on the possibility of translating some geometric properties from classical manifolds to exotic ones. More precisely, one considers diagrams like:

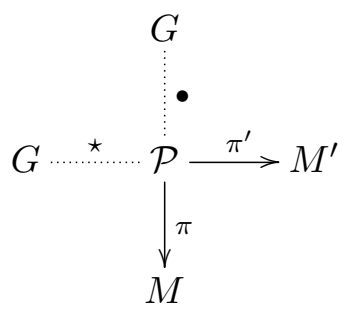

where the submersions $\pi$ and $\pi^{\prime}$ define principal bundles with respect to the commuting $\bullet, \star$ actions on $\mathcal{P}$.

In the Chapter 4, we make a systematic exposition of the general theory of these procedures, extensively exploited in [Dur01, DMR04, Spe10, Spe16a], to produce metrics of positive Ricci curvature and almost non-negative sectional curvature on several new examples of exotic manifods. The idea consists in taking a well known triple $(G, \mathcal{P}, M)$ to geometrically realize exotic manifolds (here represented by $M^{\prime}$ ). The exotic candidate $M^{\prime}$ is then recognized by some smooth invariants, which we do not exploit here.

The presentation developed in the Chapter 4 is based on my first paper [CS18c]. However, it is worth pointing out that concerning the topological realizations, the contribution was primarily given by my advisor, Prof. Sperança. That said, we shall briefly discuss these, mostly focusing on the geometrical aspects of such constructions, once these are in fact the candidate contributions. We prove:

Theorem A. Let $\Sigma^{7}$ and $\Sigma^{8}$ be any homotopy spheres of dimensions 7 and 8 , respectively; $\Sigma^{10}$ be any homotopy sphere of dimension 10 that bounds a spin manifolds; $\Sigma^{4 m+1}, \Sigma^{8 m+5}$ be any Kervaire sphere of dimensions $4 m+1,8 m+5$, respectively. Then each of the following manifolds admit a sequence of Riemannian metrics $\left\{g_{n}\right\}_{n \in \mathbb{N}}$ of positive Ricci curvature satisfying $\sec _{g_{n}} \geq-1 / n$ and $\operatorname{diam}_{g_{n}} \leq 1 / n$ :

(i) $M^{7} \# \Sigma^{7}$, where $M^{7}$ is any $S^{3}$-bundle over $S^{4}$

(ii) $M^{8} \# \Sigma^{8}$, where $M^{8}$ or a $S^{3}$-bundle over $S^{5}$ or a $S^{4}$-bundle over $S^{4}$

(iii) $M^{10} \# \Sigma^{10}$, where:

(a) $M^{10}=M^{8} \times S^{2}$ with $M^{8}$ as in item (ii)

(b) $M^{10}$ is any $S^{3}$-bundle over $S^{7}, S^{5}$-bundle over $S^{5}$ or $S^{6}$-bundle over $S^{4}$

(iv) $M^{4 m+1} \# \Sigma^{4 m+1}$ where 
(a) $S^{2 m} \hookrightarrow M^{4 m+1} \rightarrow S^{2 m+1}$ is the sphere bundle associated to any multiple ${ }^{1}$ of $O(2 m+1) \hookrightarrow$ $O(2 m+2) \rightarrow S^{2 m+1}$, frame bundle of $S^{2 m+1}$

(b) $\mathbb{C} P^{m} \hookrightarrow M^{4 m+1} \rightarrow S^{2 m+1}$ is the $\mathbb{C} P^{m}$-associated bundle to any multiple ${ }^{1}$ of unitary frame bundle $U(m) \hookrightarrow U(m+1) \rightarrow S^{2 m+1}$

(c) $M^{4 m+1}=\frac{U(m+2)}{S U(2) \times U(m)}$

(v) $\left(M^{8 r+k} \times N^{5-k}\right) \# \Sigma^{8 r+5}$ where $N^{5-k}$ is any riemannian manifold with positive Ricci curvature

(a) $S^{4 r+k-1} \hookrightarrow M^{8 r+k} \rightarrow S^{4 r+1}$ is the $k$-th suspension of the unitary tangent bundle $S^{4 r-1} \hookrightarrow$ $T^{1} S^{4 r+1} \rightarrow S^{4 r+1}$,

(b) for $k=1, \mathbb{H} P^{m} \hookrightarrow M^{8 m+1} \rightarrow S^{4 m+1}$ is the $\mathbb{H} P^{m}$-associated bundle to any multiple ${ }^{1}$ of $S p(m) \hookrightarrow S p(m+1) \rightarrow S^{4 m+1}$

(c) for $k=0, M=\frac{S p(m+2)}{S p(2) \times S p(m)}$

(d) for $k=1, M=M^{8 m+1}$ is as in item (iv).

The same techniques allow us to prove that:

Theorem B. The following exotic projective spaces $\Sigma \mathcal{P} \mathbb{C}^{n-1}, \Sigma \mathcal{P} \mathbb{H}^{m-1}$ carry $U(n / 2), S p(m / 2)$ invariant metrics, respectively, with positive Ricci curvature, where

$$
\begin{aligned}
\Sigma \mathcal{P} \mathbb{C}^{n-1} & :=\Sigma^{2 n-1} / S^{1}, \\
\Sigma \mathcal{P} \mathbb{H}^{m-1} & :=\Sigma^{4 m-1} / S U(2) .
\end{aligned}
$$

Perhaps the main reason that in the construction of manifolds with some positive curvature property, the examples are obtained via submersions, is due to the Gray-O'Neill submersion formula, since this implies that the sectional curvature of the base is greater or equal to the sectional curvature of the total space (in horizontal directions). However, it is not necessarily true that Riemannian submersions preserve positive Ricci curvature (see [PW14]).

The converse question, namely: given a Riemannian submersion with a metric of positive sectional curvature on its base, is it possible to obtain a submersion Riemannian metric with positive sectional curvature in the total space? Admits an easy counterexample. Just consider as total space the manifold $\mathbb{R} P^{2} \times \mathbb{R} P^{2}$ with the transitive $S O(3)$-action in the second factor. The basis of this submersion (which is the space of the orbits of this action) is $\mathbb{R} P^{2}$, which has positive sectional curvature in the submersion metric $S^{2} \rightarrow \mathbb{R} P^{2}$. However, by Synge's theorem, the positive curvature cannot be raised to the total space because $\mathbb{R} P^{2} \times \mathbb{R} P^{2}$ is not simply connected.

The following natural question would therefore be: the same holds for Ricci and scalar curvatures? Searle-Wilhelm in [SW15] showed that for a large class of examples, it is possible to lift positive Ricci curvature from the orbit space associated with a manifold with isometric action by a compact Lie group.

Theorem C (Searle-Wilhlem). Let $(M, g)$ be a $G$-Riemannian manifold where $M$ and $G$ are compact. Assume that

1. A principal orbit of $G$ on $M$ has finite fundamental group,

2. $\operatorname{Ric}_{M / G} \geq 1$ in the orbital distance metric.

Then $M$ carries a G-invariant metric of positive Ricci curvature.

The main idea for the proof of Theorem $\mathrm{C}$ is to perform a conformal change on the metric $g$, followed by a Cheeger deformation. Their proof is quite complicated and divided into several steps, so it gave rise to the following questions:

\footnotetext{
${ }^{1}$ i.e, a bundle such that the transitions functions $\alpha: S^{n-1} \rightarrow G$ are pointwise multiple of $\tau_{2 m}: S^{2 m} \rightarrow O(2 m+1)$, $\tau_{m}^{\mathbb{C}}: S^{2 m} \rightarrow U(m)$ or $\tau_{m}^{\mathbb{H}}: S^{4 m+2} \rightarrow S p(m)$, transitions fuctions of the orthonormal frame bundle and its reductions, respectively.
} 
Is it possible to prove this result using only Cheeger deformations? Would that simplify the proof?

In the present thesis, we answered them completely. For this purpose, we studied the behavior of the tensors associated with Cheeger deformations at singular points, that is, points where the dimension of the horizontal space (that is, the dimension of the space complementary to the orbit) is greater than in the regular part (as a result of the larger isotropy). This addition of horizontal directions is what makes it difficult to control Ricci's curvature, since the new terms could contribute with a negative sign. On the other hand, in order to get an affirmative answer, a blow-up behavior of positive amounts in the expression of Ricci's curvature was expected. We note that this blowup does occur (Corollary E and Theorem H) but in certain directions (Theorems D, F and G). Precisely:

Theorem D. Let $\left(M^{n}, g\right)$ be a compact connected riemannian manifold with an isometric action by a compact and connected Lie group $G$. Denote by $\mathcal{H}_{p}$ the horizontal space with respect to the $G$-action at $p$. Assume that:

1. A principal orbit of $G$ on $M$ has finite fundamental group,

2. $\operatorname{Ric}_{M^{\text {reg }} / G} \geq 1$

If $g$ has directions of negative Ricci curvature for each Cheeger deformation, then

(a) There exists a singular point $p \in M$ and a non-zero vector $X \in \mathcal{H}_{p}$ that is fixed by the istropy representation $\rho: G_{p} \rightarrow O\left(\mathcal{H}_{p}\right)$,

(b) The restriction of $\rho$ to $X^{\perp} \cap \mathcal{H}_{p}$ is reducible.

Moreover, if $X^{\perp} \cap \mathcal{H}_{p}$ has exactly two $\rho$-irreducible summands, $X^{\perp} \cap \mathcal{H}_{p}=\mathcal{H}_{1}+\mathcal{H}_{2}$, then (up to changing the order of the summands), for any regular vector $Y=Y_{1}+Y_{2} \in \mathcal{H}_{1}+\mathcal{H}_{2}$,

$$
\operatorname{dim} \mathcal{H}_{1}-\operatorname{dim} G_{p} Y_{1}>\frac{(k-1) \operatorname{dim} \mathcal{H}_{1}}{\operatorname{dim} \mathcal{H}_{p}-1}
$$

where $k$ is the codimension of a principal orbit.

In particular, in this case, where Cheeger's deformations are ineffective, there is a geodesic on $M$ that is entirely contained in the singular stratum. As a natural consequence, we have:

Corollary E. Let $(M, g)$ be a compact connected Riemannian manifold with an isometric action by a compact connected Lie group. Assume that:

1. A principal orbit of $G$ on $M$ has finite fundamental group,

2. $\operatorname{Ric}_{M^{r e g} / G} \geq 1$,

Then $g$ develops positive Ricci curvature after a finite Cheeger deformation if one of the following equivalent conditions is satisfied

(a) The singular strata is composed by isolated orbits;

(b) For every singular point $p$, the linear isotropy representation in this point is irreducible;

(c) No istropy representantion has a non-zero fixed vector;

(d) The induced $G$-action in the unitary tangent space $T_{1} M$ has no fixed points.

We also obtained a local converse for Theorem D:

Theorem F. Let $G$ be a compact connected Lie group acting on a compact manifold $M$. Assume that: 
$\left(a^{\prime}\right)$ there exists a fixed point $p$ such that the isotropy representation, $\rho: G_{p} \rightarrow O\left(\mathcal{H}_{p}\right)$, fix a non-zero vector $X \in \mathcal{H}_{p}$,

(b') $X^{\perp} \cap \mathcal{H}_{p}$ decomposes into two $\rho$-invariant subspaces, $X^{\perp} \cap \mathcal{H}_{p}=\mathcal{H}_{1}+\mathcal{H}_{2}$, satisfying

$$
\operatorname{dim} \mathcal{H}_{1}-\operatorname{dim} G_{p} Y_{1}>\frac{(k-1) \operatorname{dim} \mathcal{H}_{1}}{\operatorname{dim} \mathcal{H}_{p}-1}
$$

for every regular vector $Y \in X^{\perp} \cap \mathcal{H}_{p}$.

Then there exists a $G$-invariant neighbourhood of $p$ and a $G$-invariant metric $g$ on $M$ satisfying

1. $\operatorname{Ric}_{M^{r e g} / G} \geq 1$

2. $\operatorname{Ric}_{g_{t}}(X)<0$ for every Cheeger deformation $g_{t}$ of $g$

It is worth noting that Theorem $\mathrm{F}$ does not assume that $\mathcal{H}_{1}, \mathcal{H}_{2}$ are the only non-trivial invariant subspaces of $\left.\rho\right|_{X \perp \cap \mathcal{H}_{p}}$.

The algebraic nature of our results follows from the fact that: from [SW15, Proposition 3.1], we know that vectors with negative Ricci curvature can only occur at singular points. Furthermore, the estimates presented in Section 5.2 guarantee that such vectors are fixed by the isotropy representation at its base point. So, given such a vector $X \in \mathcal{H}_{p}$, Schur's Lemma guarantees that the restriction of $R_{X}(Y)=R(Y, X) X$ to each $\rho$-reducible subspace is a multiple of identity. The inequalities in $\mathrm{D}$ and $\mathrm{F}$ follow combinatorial arguments (see, for example, the subsections 5.2.3 and $5.2 .5)$.

As a result of all the developed techniques, it was possible to build a family of global examples where Cheeger's deformations are ineffective.

Theorem G. Let $S^{n}$ be the unitary sphere in $\mathbb{R}^{n+1}$ with the standard $S O(n-2)$-action that fixes the first 3 coordinates. Then, for each $n \geq 5$, there exist a $S O(n-2)$-invariant metric $g$ on $S^{n}$ satisfying:

1. $\operatorname{Ric}_{M^{r e g} / G} \geq 1$

2. There is a non-zero vector $X \in \mathcal{H}_{p}$ such that $\operatorname{Ric}_{g_{t}}(X)<0$ for every Cheeger deformation $g_{t}$.

In addition, the estimates of the singular points allowed to present a much simpler demonstration for the classic result in [LS74] with a small improvement.

Theorem H. Let $(M, g)$ be a compact Riemannian manifold with an isometric action by a compact connected and non-Abelian Lie group $G$. Then $g$ develops positive scalar curvature after a finite Cheeger deformation.

Finally, we give a new proof (in a much simpler way) for Theorem C (Theorem 5.4.1).

In the subsection 5.6, we extend the concept of Cheeger deformation to the context of bundles with a compact structural group. If $F \hookrightarrow M \stackrel{\pi}{\rightarrow} B$ is a smooth bundle with structure group $G$, let $\mathcal{P}$ be the principal bundle associated to $\pi$. Recall that there is a Riemannian submersion $\bar{\pi}:\left(\mathcal{P} \times F, g_{\mathcal{P}} \times g_{F}\right) \rightarrow(M, h)$, where $g$ and $g_{F}$ are $G$-invariants metrics on $\mathcal{P}$ and $F$, respectively.

The idea is to perform a Cheeger deformation on the $G \times G$-manifold $\mathcal{P} \times F$. From the submersion $\bar{\pi}$ one obtains a family of Riemannian metrics $h_{t}$ on $M$. We prove that:

Theorem I. Let $F \hookrightarrow M \stackrel{\pi}{\rightarrow} B$ be a fiber bundle with compact and connected base $B$, fiber $F$ and structure group $G$. Assume that:

1. The orbits of $G$ on $\mathcal{P}$ have positive sectional curvature on a bi-invariant metric;

2. $\mathcal{P}$ admits a sequence of Riemannian metrics $g_{n}$ of positive Ricci curvature such that $K_{g_{n}}\left(X^{\mathcal{H}}, Y\right) \geq$ $-\frac{1}{n}, \forall X, Y \in T \mathcal{P}$, and $\operatorname{diam}\left(g_{n}\right) \leq \frac{1}{n}$ 
3. $g_{F}$ has a $G$-invariant metric of non-negative sectional curvature and positive Ricci curvature;

Then $M$ admits a sequence of Riemannian metrics $\widetilde{g}_{n}$ of positive Ricci curvature such that $\operatorname{diam}\left(\widetilde{g}_{n}\right) \leq$ $\frac{1}{n}, \sec \left(\widetilde{g}_{n}\right) \geq-\frac{1}{n}$.

As final contributions, we studied the problem of prescribing scalar curvature metrics in certain Riemannian submersions, being a natural extension of the classic Kazdan-Warner problem (see [KW75b], [KW75a], [KW75c]). We prove that:

Theorem J. Let $F \hookrightarrow M \stackrel{\pi}{\rightarrow} B$ be a fiber bundle where $M, F, B, G$ are compact. Assume that

1. A principal orbit of $G$ on $F$ has finite fundamental group,

2. F carries a G-invariant metric such that $\operatorname{Ric}_{F^{r e g} / G} \geq 1$.

Then

1. There is $\lambda \in(0,1]$, depending only on the geometry of the fiber $F$, such that any smooth function $f: M \rightarrow \mathbb{R}$ satisfying $\frac{\min _{p \in B} f}{\max _{p \in B} f} \leq \lambda$ is the scalar curvature of some Riemannian submersion metric on $M$, except maybe if $f=$ cte $\geq 0$;

2. If $F$ has constante scalar curvature, then any smooth function $f: M \rightarrow \mathbb{R}$ is the scalar curvatura function of some Riemannian submersion metric on $M$, except maybe if $f=$ cte $\geq 0$.

In the case where the fiber has points with non-positive scalar curvature, we follow an approach via variational methods, obtaining the following results. Define the constants

$$
b_{k}:=\frac{k+1}{8 k}, c_{k}:=\frac{(k+1)^{2}}{8(k-1) k}, \theta:=\frac{2(k-1)}{k+1} .
$$

Theorem K. Let $\pi:\left(F^{k}, g_{F}\right) \hookrightarrow\left(M^{n+k}, g\right) \rightarrow\left(B^{n}, h\right)$ be a Riemannian submersion where $M$ is closed and connected and $F$ is compact and connected. Assume that:

1. $\min _{F} \operatorname{scal}_{F} \leq 0$,

2. $\left(F, g_{F}\right)$ is a minimal submanifold of $(M, g)$,

3. If A denotes the Gray-O'Neill tensor of the submersion $\pi$, then

$$
\max _{M}\left\{3 \sum_{i, j}\left|A_{e_{i}} e_{j}\right|^{2}-2 \sum_{i, r}\left|A_{e_{i}}^{*} v_{r}\right|^{2}\right\} \leq 0,
$$

where $\left\{e_{i}\right\}$ is a g-orthonormal basis for $\mathcal{H}$ and $\left\{v_{i}\right\}$ is a g-orthonormal basis for $\mathcal{V}$.

Then any smooth function $f \in C^{\infty}(M ; \mathbb{R})$ satisfying

$$
\frac{\lambda_{1}}{2+\epsilon}-b_{k} \max _{M}\left(f-\mathrm{scal}_{g}+\operatorname{scal}_{F}\right)+c_{k} \min _{F} \operatorname{scal}_{F}(\operatorname{vol}(B))^{2 / \theta-1}+b_{k} \min _{M} \delta A>0,
$$

for some $\epsilon>0$, where $\lambda_{1}$ denotes the first positive eigenvalue of $-\Delta_{B}$, is the scalar curvature for some Riemannian submersion metric on $M$.

If $M$ is isometric to a product, once its fibers are totally geodesic submanifolds and $A \equiv 0$ one has

Theorem L. Let $M=\left(B^{n} \times F^{k}, g_{B} \times g_{F}\right)$ be a closed and connected Riemannian manifold. Suppose that $\min _{F} \operatorname{scal}_{F} \leq 0$. Then any smooth function $f \in C^{\infty}(M ; \mathbb{R})$ satisfying

$$
\frac{\lambda_{1}}{2+\epsilon}-b_{k} \max _{B}\left(f-\operatorname{scal}_{B}\right)+c_{k} \min _{F} \operatorname{scal}_{F}(\operatorname{vol}(B))^{2 / \theta-1}>0, \exists \epsilon>0,
$$


for some $\epsilon>0$, where $\lambda_{1}$ denotes the first positive eigenvalue of $-\Delta_{B}$, is the scalar curvature for some Riemannian submersion metric on $M$.

Theorems 7.2.1 and 7.2.2 are natural generalizations of the problem of prescribing constant scalar curvature via warped products, largely studied in[DD87], [EYTK96]. Under the hypothesis of positive Ricci curvature on $B$ and $M$ being isometric to a product one has another condition:

Theorem M. Let $M^{n+k}=\left(B^{n} \times F^{k}, g_{B} \times g_{F}\right)$. Assume that $\operatorname{Ric}\left(g_{B}\right) \geq(n-1)$ and that $\min _{F} \operatorname{scal}_{F} \leq 0$. Then any smooth function $f: M \rightarrow \mathbb{R}$ such that

$$
n\left(\frac{8 k}{(2+\epsilon)(k+1)}+(n-1)\right)>\max _{B} f+\frac{c_{k}}{b_{k}} \min _{F} \operatorname{scal}_{F}(\operatorname{vol}(B))^{2 / \theta-1},
$$

for some $\epsilon>0$, is the scalar curvature function for some Riemannian submersion metric on $M$.

Finally, we obtained a necessary and sufficient condition for a $G$-manifold to admit a metric of positive sectional curvature (Theorem N); and in collaboration with the co-advisor Prof. Alexandrino, we obtained some results regarding the Mean Curvature Flow of Singular Riemannian Foliations (Theorem O):

Theorem N. Let $(M, g)$ be a compact manifold with an isometric action by a compact and connected Lie group G. Fix a bi-invariant metric $Q$ on $G$. Assume that for each $p \in M^{r e g}, G / G_{p}$ has positive sectional curvature on the normal homogeneous space metric. Then there is $t>0$ such that the Ceeger deformation $g_{t}$ of $g$ satisfies $\sec _{g_{t}}>0$ if, and only if, there is $k>0$ such that

$$
\begin{array}{r}
\left(K_{B}(X, Y)-k|X \wedge Y|_{g}^{2}\right)\left(\frac{1}{2} Q\left(\operatorname{Hess} P^{-1}(X) V, V\right)+\frac{1}{4}\left|\Omega_{X}^{*} V\right|_{g}^{2}-k|X|_{g}^{2} Q\left(P^{-1} V, V\right)\right) \\
\geq \frac{1}{4} Q\left(\left(\nabla_{X} \Omega\right)_{X} Y, V\right)^{2}
\end{array}
$$

for every $X, Y \in \mathcal{H}_{p}, V \in \mathfrak{m}_{p}, p \in M^{r e g}$, where $\mathfrak{m}_{p}$ is identified with the tangente space to the orbit of $G$ through $p ; P$ is the unique symmetric and positive definite tensor satisfying $g(V, W)=Q(P V, W)$ and $\Omega$ is the curvature 2 -form of the principal bundle

$$
M^{r e g} \rightarrow M^{r e g} / G
$$

Theorem O. Let $(M, g)$ be a complete Riemannian manifold with a Singular Riemannian Foliation $\mathcal{F}$ with closed leaves. Assume that

1. M has bounded sectional curvature;

2. The shape operator along each leaf $L \in \mathcal{F}$ is bounded;

3. The quotient $M / \mathcal{F}$ is compact.

Let $N$ be an immersed submanifold contained in a regular leaf. Assume that the dimension of $N$ is greater than the dimension of any singular leaf and that the Mean Curvature Flow $N(t)$ is the restriction of a basic flow (with respect to $\mathcal{F}$ ) in the regular stratum, with maximum interval of existence $[0, T), T<\infty$. Then $N(t)$ converges to a singular leaf in the leaf space sense. 


\section{Capítulo 3}

\section{Algumas deformações métricas e aplicações}

\subsection{Folheações métricas}

O propósito desta seção é permitir que esta tese seja o máximo possível autocontida, e para que deixemos claras as notações e convenções assumidas ao longo de todo o texto. Assumiremos que o leitor entenda bem sobre fibrados vetoriais, variedades suaves, Grupos de Lie, geometria riemanniana clássica e alguma topologia algébrica. Para detalhes sobre esses conteúdos nos referimos à [Lee], [Pet06a], [FdC13], [Bre93], respectivamente.

Folheações riemannianas dividem-se essencialmente em: regulares e singulares. Para motivar a primeira dessas subclasses, relembraremos rapidamente o conceito de submersões riemannianas, exemplificadas por fibrados (com fibra qualquer), provando, ou apenas referenciando, alguns resultados que serão úteis para o restante do texto. Destacamos como principais referências [KN63, GW09]. Para a segunda classe, daremos destaque especial para variedades riemannianas munidas de uma ação isométrica (não necessariamente livre) por um grupo de Lie compacto e conexo, baseando-nos majoritamente em [Bre72, AB15, Rad].

\subsubsection{Submersões riemannianas e fibrados topológicos}

Sejam $M, N$ variedades suaves. Uma submersão entre $M$ e $N$ consiste de uma aplicação $\pi$ : $M \rightarrow N$ tal que, para todo $p \in M$, a aplicação diferencial $d \pi(p): T_{p} M \rightarrow T_{\pi(p)} N$ seja linear e sobrejetiva. Dessa maneira, todo ponto $p \in M$ é regular, no sentido do Teorema 5.22, p. 113 em [Lee]. Pelo Teorema da Aplicação Inversa ([Lee, Theorem 5.11, p. 105]), $\pi^{-1}(p)$ consiste em uma subvariedade mergulhada em $M$ cujo espaço tangente é igual ao ker $d \pi(p)$. Assim, a coleção $\mathcal{F}:=\left\{f^{-1}(p)\right\}$ particiona $M$ em subvariedades de mesma dimensão e leva o nome de folheação. Naturalmente, cada subvariedade $f^{-1}(p)$ é chamada de folha ou fibra.

Em cada ponto $p \in M$, o subspaço vetorial $\operatorname{ker} d \pi(p) \subset T_{p} M$ é chamado de espaço vertical em $p$ associado a folheação $\mathcal{F}$, sendo denotado por $\mathcal{V}_{p}$. Ainda mais, $\mathcal{V}:=\bigcup_{p} \mathcal{V}_{p}$ tem estrutura de subfibrado vetorial de $T M$.

Um problema natural consiste em procurar por um subfibrado complementar a $\mathcal{V}$ em $T M$. Com esse fim, assumiremos que $M$ esteja munida de uma métrica riemanniana completa $g$. Nesse caso, uma escolha óbvia para tal complemento consiste em colecionar os espaços vetoriais $\left\{\operatorname{ker} d \pi(p)^{\perp}:=\right.$ $\left.\mathcal{H}_{p}\right\}_{p \in M}$ e definir $\mathcal{H}:=\bigcup_{p} \mathcal{H}_{p}$. Como para cada $p \in M$ tem-se $\mathcal{H}_{p} \subset T_{p} M$ e a aplicação $p \mapsto \operatorname{dim} \mathcal{H}_{p}$ é constante, pode-se definir uma estrutura de subfibrado vetorial em $\mathcal{H}$ de tal maneira que $T M=$ $\mathcal{H} \oplus \mathcal{V}$.

Note ainda que a restrição $\left.d \pi(p)\right|_{\mathcal{H}_{p}}: \mathcal{H}_{p} \rightarrow T_{\pi(p)} N$ é um isomorfismo. Assim, fica bem definida uma aplicação inversa a essa $\mathcal{L}_{\pi(p)}: T_{\pi(p)} N \rightarrow \mathcal{H}_{p}$, chamada de lenvantamento horizontal por $\pi$ em $p$. 
Definição 1. Se $N$ está munida de uma métrica riemanniana $h$ tal que

$$
\left.d \pi(p)\right|_{\mathcal{H}_{p}}:\left(\mathcal{H}_{p}, g_{p}\right) \rightarrow\left(T_{\pi(p)} N, h_{\pi(p)}\right)
$$

seja uma isometria para cada $p$, dizemos que $\pi$ é uma submersão riemanniana e, nesse caso, $\mathcal{F}$ é dita folheação riemanniana regular.

Notamos portanto que uma submersão $\pi:(M, g) \rightarrow(N, h)$ entre variedades riemannianas é uma submersão riemanniana se, e somente se, o levantamento horizontal $\mathcal{L}_{\pi(p)}$ for uma isometria para todo $p \in M$.

Subfibrados de $T M$ tais como $\mathcal{V}$ e $\mathcal{H}$ são chamados de distribuições. Dada uma distribuição $\mathcal{D}$ em $T M$, uma seção local consiste em uma aplicação suave $s: U \subset M \rightarrow \mathcal{D}$, com $U \subset M$ aberto, tal que $\pi \circ s=1_{U}$, onde $1_{U}$ denota a aplicação identidade em $U$. Em particular, para quaquer variedade suave $M$, campos de vetores em $M$ são seções de $T M$.

Seja $\mathcal{D} \subset T M$ uma distribuição. Note que podemos restringir o colchete de Lie de campos $[\cdot, \cdot]$ para elementos de $\mathcal{D}$. Isso faz com que $\mathcal{D}$ seja uma álgebra de campos.

Definição 2. Se a álgebra de campos $(\Gamma(\mathcal{D}),[\cdot, \cdot])$ for uma subálgebra de Lie de $(\Gamma(T M),[\cdot, \cdot])$, dizemos que $\mathcal{D}$ é uma distribuição integrável.

Definição 3. 1. Um campo de vetores $V \in \Gamma(T M)$ é dito vertical se for uma seção de $\mathcal{V}$. Denotamos o conjunto de tais campos por $\Gamma(\mathcal{V})$.

2. Um campo de vetores $X \in \Gamma(T M)$ é dito horizontal se for uma seção de $\mathcal{H}$. Denotamos o conjunto de tais campos por $\Gamma(\mathcal{H})$.

3. Um campo de vetores $E \in \Gamma(T M)$ é dito projetável se $d \pi(p)(E)=d \pi\left(p^{\prime}\right)(E)$ para todos $q \in N, p, p^{\prime} \in \pi^{-1}(q)$.

4. Um campo de vetores $E$ em $M$ é dito básico se for horizontal e projetável. Denotamos o conjunto de tais campos por $\Gamma(\mathcal{H})^{\pi}$.

O subespaço $\Gamma(\mathcal{H})^{\pi} \subset \Gamma(\mathcal{H})$ é isomorfo ao espaço de campos vetoriais $\Gamma\left(\pi^{*}(T N)\right)$. Entretanto, vale notar que $\Gamma(\mathcal{H})^{\pi}$ não é, em geral, uma álgebra de Lie, embora a seguinte relação seja verdadeira

$$
[X, V]^{\mathcal{H}}=0, \forall X \in \Gamma(\mathcal{H})^{\pi}, \forall V \in \Gamma(\mathcal{V}) .
$$

A decomposição $T M=\mathcal{H} \oplus \mathcal{V}$ reflete também na conexão, de modo que $\nabla^{\mathcal{V}}$ denotará a componente vertical da conexão riemanniana $\nabla$ associada a $g$, e $\nabla^{\mathcal{H}}$ denotará a componente horizontal dessa conexão. Precisamente:

$$
\nabla_{X}^{\mathcal{H}} Y=\left(\nabla_{X} Y\right)^{\mathcal{H}} \text { e } \nabla_{X}^{\mathcal{V}} Y=\left(\nabla_{X} Y\right)^{\mathcal{V}}
$$

para todos $X, Y \in T_{p} M$, para todo $p \in M$.

Nas próximas linhas, com o intuito de enunciar as fórmulas de curvatura de Gray-O'Neill, recordaremos rapidamente quem são os tensores fundamentais associados às submersões riemannianas. No que segue, assumimos que $\pi:(M, g) \rightarrow(N, h)$ seja uma submersão riemanniana.

Definição 4 (Tensor de Gray-O'Neill). O campo tensorial $A: \Gamma(\mathcal{H}) \times \Gamma(\mathcal{H}) \rightarrow \Gamma(\mathcal{V})$ definido por

$$
A_{X} Y:=\nabla_{X}^{\mathcal{V}} Y=\frac{1}{2}[X, Y]^{\mathcal{V}}
$$

é dito Tensor de Gray-O'Neill.

O mapa $A$ definido por (3.3) é de fato um tensor, de modo que só depende dos valores de $X$ e $Y$ no ponto calculado. Note por exemplo que se $f: M \rightarrow \mathbb{R}$ é uma função suave, então para quaisquer 
$X, Y \in \Gamma(\mathcal{H})$ temos

$$
[f X, Y]^{\mathcal{V}}=(f X Y-d f(Y) X-f Y X)^{\mathcal{V}}=f[X, Y]^{\mathcal{V}} .
$$

Nota-se que de acordo com Teorema de Frobenius ([AB15, Theorem A.19, p. 191]), o tensor $A$ mede a falta de integrabilidade da distribuição horizontal.

Será muito frequente também considerar o operador $A_{X}^{*}$ dual a $A_{X}$ :

$$
g\left(A_{X} Y, V\right)=g\left(A_{X}^{*} V, Y\right), \forall X, Y \in \Gamma(\mathcal{H}), V \in \Gamma(\mathcal{V}) .
$$

Definição 5. O operador formato, denotado por $S$, consiste no campo tensorial $S: \Gamma(\mathcal{H}) \times \Gamma(\mathcal{V}) \rightarrow$ $\Gamma(\mathcal{V})$ definido por

$$
S_{X} U:=-\nabla_{U}^{\mathcal{V}} X
$$

Será conveniente considerar seu tensor dual

$$
\sigma(U, V):=\nabla_{U}^{\mathcal{H}} V, g\left(S_{X} U, V\right)=g(\sigma(U, V), X), \forall U, V \in \mathcal{V}, X \in \mathcal{H}
$$

denominado tensor de segunda forma fundamental das folhas de $\mathcal{F}$, cuja nomenclatura e equação (3.5) caracterizam sua interpretação geométrica. Precisamente:

Definição 6. Se $S \equiv 0$ dizemos que as folhas de $\mathcal{F}$ são totalmente geodésicas.

Como o operador formato $S$ está diretamento ligado ao tensor de segunda forma fundamental das fibras, introduzimos a 1-forma de curvatura média de uma fibra $\pi^{-1}(p)$, definida via a aplicação

$$
\kappa(X):=\operatorname{tr} S_{X}, X \in \mathcal{H}_{p}
$$

O vetor de curvatura média $H$ de $\pi^{-1}(p)$ é definido unicamente pela expressão

$$
\kappa(X)=g(X, H), \forall X \in \mathcal{H}_{p} .
$$

Ademais, se $X, Y$ são campos básicos, então

$$
d \kappa(X, Y)=\operatorname{tr} d^{\nabla} S_{X, Y}=\operatorname{tr}\left(\nabla_{X} S\right)_{Y}-\left(\nabla_{Y} S\right)_{Y} .
$$

O hessiano da função $\phi$ consiste na forma bilinear simétrica

$$
\text { Hess } \phi(X, Y)=g\left(\nabla_{X} \nabla \phi, Y\right) \text {. }
$$

O laplaciano de $\phi$ é dado por

$$
\Delta \phi=\operatorname{tr}: X \mapsto \operatorname{Hess} \phi(X, X)
$$

Em particular,

$$
\Delta \phi=\Delta_{B} \phi-\kappa(H)=\Delta_{B} \phi-g(\nabla \phi, H) .
$$

Convenção. Assumiremos a seguinte convenção para o Tensor de Riemann $R_{g}(\cdot, \cdot) \cdot$ de $\nabla$ :

$$
R_{g}\left(E, E^{\prime}\right) F:=\nabla_{E} \nabla_{E^{\prime}} F-\nabla_{E^{\prime}} \nabla_{E} F-\nabla_{\left[E, E^{\prime}\right]} F .
$$

Assim, a curvatura seccional de um plano $\{E, F\}$ é definida como

$$
K_{g}(E, F):=\frac{R_{g}(E, F, F, E)}{|E \wedge F|^{2}}:=\frac{g\left(R_{g}(E, F) F, E\right)}{|E \wedge F|^{2}}
$$

onde $|E \wedge F|^{2}:=|E|^{2}|F|^{2}-g(E, F)^{2}$.

Teorema 3.1.1 (Fórmulas de Curvatura de Gray-O'Neill). Fixe um ponto $p \in M$ e vetores ortonormais $X, Y \in \mathcal{H}_{p}, V, U \in \mathcal{V}_{p}$. Denote por $K_{g}, K_{h}, K^{F}$ as curvaturas seccionas de $(M, g),(N, h),\left(F, g_{F}\right)$, 
respectivamente, onde $F$ é uma folha da submersão $\pi$ e $g_{F}$ é a métrica de imersão isométrica induzida por $g$ em F. Então as seguintes equações são verdadeiras:

$$
\begin{aligned}
K_{g}(d \pi(p) X, d \pi(p) Y) & =K_{h}(X, Y)+3\left|A_{X} Y\right|^{2} ; \\
K_{g_{F}}(V, U) & =K_{g}(U, V)+\sigma(U, U) \sigma(V, V)-\sigma^{2}(U, V) ; \\
K_{g}(X, V) & =g\left(\left(\nabla_{X}^{\mathcal{V}} S\right)_{X} V, v\right)+\left|A_{X}^{*} V\right|^{2}-\left|S_{X} V\right|^{2} .
\end{aligned}
$$

Demonstração. Veja [GW09, p. 24-28].

Exemplos de submersões riemannianas de grande interesse para esta tese consistem em fibrados principais e fibrados associados. Porém, para devidamente introduzi-los, vamos relembrar alguns conceitos, propriedades e resultados referentes a ações (suaves) de Grupos de Lie em variedades. Indicamos como principais referências [Bre72] e [AB15].

Definição 7. Seja $M$ uma variedade suave e $G$ um grupo de Lie. Dizemos que $G$ age de maneira suave em $M$ se existir uma representação (homomorfismo de grupos)

$$
\rho: G \rightarrow \operatorname{Diff}(M),
$$

tal que a aplicação induzida $\rho: G \times M \rightarrow M$ seja uma função suave, onde $\operatorname{Diff}(M)$ denota o grupo de difeomorfismos de $M$ (com operação de multiplicação dada pela composição).

Se $M$ está munido de uma métrica riemanniana $g$, dizemos que $G$ age isometricamente em $(M, g)$ se existir uma representação (homomorfismo)

$$
\rho: G \rightarrow \operatorname{Iso}(M, g),
$$

onde $\rho$ é uma aplicação suave e $\operatorname{Iso}(M, g)$ denota o grupo que consiste nas isometrias de $(M, g)$.

Definição 8. Uma variedade suave (resp. riemanniana) munida de uma ação suave $\rho: G \rightarrow$ $\operatorname{Diff}(M)$ (isométrica $\rho: G \rightarrow \operatorname{Iso}(M, g)$ ) será dita uma $G$-variedade (resp. riemanniana).

Seja $M$ uma $G$-variedade (suave ou riemanniana) cuja ação é denotada por $\rho$. Dados $g, g^{\prime} \in$ $G$, dizemos que a ação por $G$ é à esquerda se para cada $p \in M, \rho(g) \circ \rho\left(g^{\prime}\right)(p)=\rho\left(g g^{\prime}\right)(p)$. Reciprocamente, dizemos que a ação por $G$ é à direita se para cada $p \in M, \rho(g) \circ \rho\left(g^{\prime}\right)(p)=$ $\rho\left(g^{\prime} g\right)(p)$.

Convenção. 1. Ao longo de todo texto, salvo quando necessário explicitar a representação, denotaremos a ação à esquerda de $G$ em um ponto $p \in M$ simplesmente por $g \cdot p$ ou gp. Analogamente, a ação à direita por $g$ em $p$ será denotada por pg ou p.g.

2. O elemento neutro de $G$ será denotado por "e".

Definição 9. Seja $M$ uma $G$-variedade cuja ação é denotada por $\rho$. Nesse caso dizemos que

(a) $\rho$ é efetiva se para cada $g \neq e \in G$ existir $p \in M$ tal que $g p \neq p$. Equivalentemente, $\rho$ é um monomorfismo de grupos.

(b) $\rho$ é livre se para cada $p \in M$, gp $=p$ implicar que $g=e$,

(c) $\rho$ é transitiva se para dados $p, q \in M$ existir $g \in G$ tal que $p=g q$.

Definição 10. Seja $M$ uma $G$-variedade (com ação à esquerda). Fixe $p \in M$.

1. O conjunto $G_{p}:=\{g \in G: g p\}$ é um subgrupo de Lie de $G$ denominado subgrupo de isotropia em $p$.

2. O conjunto $G p=\{g p: g \in G\}$ é denominado órbita de $G$ em $p$. 
A ação de $G$ restrita ao conjunto $G p$ é transitiva, de modo que duas órbitas coincidem ou são disjuntas. Dessa maneira, dados $p, q \in M$, introduzimos a relação de equivalência $p \sim q \Leftrightarrow G p=G q$. O espaço quociente $M / \sim$ coleciona as órbitas da ação de $G$ em $M$ e será denotado por $M / G$.

Proposição 3.1.2. Se G é compacto e $M$ é uma G-variedade então,

1. Para cada $p \in M$, a órbita Gp é subvariedade mergulhada em $M$,

2. Gp é difeomorfa a $G / G_{p}$,

3. A aplicação no quociente $M \rightarrow M / G$ é fechada,

4. $M / G$ é um espaço topológico Hausdorff tal que $M$ é compacta se, e somente se, $M / G$ o for.

Demonstração. Veja [Bre72, Theorem 3.1, p. 38] e [AB15, Proposition 3.41, p. 62].

Convenção. Sejam $M$ uma variedade suave e $G$ um Grupo de Lie. A partir de agora, mudamos um pouco a nomenclatura e dizemos que $M$ é uma G-variedade (suave ou riemanniana) se a ação por $G$ (suave ou isométrica) também for efetiva.

Recordamos rapidamente que dado um grupo de Lie $G$, o espaço tangente à identidade, $T_{e} g:=\mathfrak{g}$, admite um único produto bilinear $[\cdot, \cdot]: \mathfrak{g} \times \mathfrak{g} \rightarrow \mathfrak{g}$ tal que

$$
\begin{gathered}
{[U, U]=0, \forall U \in \mathfrak{g}, \quad \text { alternatividade }} \\
{[[U, V], W]+[W,[U, V]]+[V,[W, U]]=0, \forall U, V, W \in \mathfrak{g}, \quad \text { identidade de Jacobi. }}
\end{gathered}
$$

Seguem ainda dessas propriedades que $[U, V]=-[V, U], \forall U, V \in \mathfrak{g}$.

Definição 11. Ao par $(\mathfrak{g},[\cdot, \cdot])$ damos o nome de álgebra de Lie de $G$.

Para cada $g \in G$, existem aplicações suaves de translações à direita e à esquerda, respectivamente dadas por

$$
\begin{aligned}
& R_{g}(h):=h g \\
& L_{g}(h)=g h .
\end{aligned}
$$

Em particular, tomando as diferenciais, obtemos as seguintes aplicações lineares $\left(d R_{g}\right)_{h}: T_{h} G \rightarrow$ $T_{h g} G$ e $\left(d L_{g}\right)_{h}: T_{h} G \rightarrow T_{g h} G$. Assim, se $c:(-\epsilon, \epsilon) \rightarrow G$ é uma curva suave tal que $c(0)=e, c^{\prime}(0)=$ $U$, então para qualquer $g \in G$ tem-se que $L_{g}(c) \equiv g c$ é uma curva suave em $G$. Logo,

$$
\left.\frac{d}{d t}\right|_{t=0} L_{g}(c(t))=\left.\frac{d}{d t}\right|_{t=0} g c(t)=\left(d L_{g}\right)_{e}(U)=g U
$$

Portanto, a multiplicação em $G$ define, via derivada da translação à esquerda, uma ação de $G$ em $\mathfrak{g}$. Dessa maneira, dado um produto interno $\langle\cdot, \cdot\rangle$ em $\mathfrak{g}$, dizemos que esse é invariante à esquerda se para todos $g \in G$ e $U, V \in \mathfrak{g}$ tivermos

$$
\langle g U, g V\rangle=\langle U, V\rangle
$$

Logo, podemos induzir uma métrica riemanniana invariante à esquerda em $G$ tomando translações de produtos internos invariantes à esquerda em $\mathfrak{g}$. Mais geralmente, não é difícil verificar que existe uma correspondência um a um entre esses objetos.

Assumindo que $G$ seja também compacto e conexo, podemos associar a esse uma métrica riemanniana bi-invariante, denotada por $Q$. Precisamente, dada uma $n$-forma invariante à esquerda em $G$, pode-se mostrar que essa é também invariante à direita. De fato, isso segue pois para cada $g \in G$, se $R_{g}: G \rightarrow G$ denota a aplicação de multiplicação à direita por $g$, então $R_{g}^{*}(\omega)$ é invariante à esquerda. Assim, $R_{g}^{*}(\omega)=f(g) \omega$ para alguma aplicação $f: G \rightarrow \mathbb{R} \backslash\{0\}$. Pode-se verificar que $f$ é 
de fato um homomorfismo (contínuo) de grupos. Como $G$ é compacto e conexo, segue que $f(G)=1$. Daí, $R_{g}^{*}(\omega)=\omega$.

Assim, se $\langle\cdot, \cdot\rangle$ é uma métrica invariante à esquerda em $G$ e $\omega$ é uma $n$-forma positiva e invariante à esquerda em $G$, a métrica $Q$ pode ser obtida via a relação:

$$
Q(U, V):=\int_{G}\langle r U, r V\rangle \omega(r), U, V \in T_{s} G, r, s \in G .
$$

Sejam $G$ um grupo de Lie compacto e conexo e $M$ uma $G$-variedade (com ação à esquerda). Lembramos que existe uma correspondência um a um entre elementos em $\mathfrak{g}=T_{e} G$ e campos invariantes à esqueda em $G$. Precisamente, essa é dada por

$$
\mathfrak{g} \ni U \mapsto U(g):=\left(d L_{g}\right)_{e} U .
$$

Para notar a boa definição do mapa, veja que dado $h \in G$, temos

$$
h U(g)=\left(d L_{h}\right)_{g}(U(g))=\left(d L_{h}\right)_{g} \circ\left(d L_{g}\right)_{e} U=d\left(L_{h g}\right)_{e} U=U(h g) .
$$

Se $U(g)$ é o campo invariante à esquerda tal que $U(e)=U$, então seu fluxo, isto é, a solução do problema de valor inicial

$$
\left\{\begin{array}{l}
\frac{d}{d t} \varphi(t)=U(\varphi(t)) \\
\varphi(0)=e
\end{array}\right.
$$

tem domínimio $\mathbb{R}$ e define uma aplicação suave entre $\mathfrak{g}$ e $G$, a saber

$$
\exp : x \mapsto \varphi(1)
$$

denominada mapa exponencial.

Pela regra da cadeia e unicidade de soluções de EDO's, pode-se mostrar que $\varphi(t)=\exp (t X)$. Portanto, fixe $U \in \mathfrak{g}, p \in M$ e denote por exp : $\mathfrak{g} \rightarrow G$ a aplição exponencial de $G$. Então, dado $\epsilon>0$, para todo $t \in(-\epsilon, \epsilon)$, $\exp (t U)$ define uma curva suave $\gamma:(-\epsilon, \epsilon) \rightarrow M$ dada por

$$
\gamma(t):=\exp (t U) \cdot p
$$

Em particular, $\gamma(0)=p$. Assim, o vetor tangente a $p$ dado por

$$
U^{*}(p)=\left.\frac{d}{d t}\right|_{t=0} \gamma(t)=\left.\frac{d}{d t}\right|_{t=0} \exp (t U) \cdot p
$$

é denominado vetor de ação em $p$ associado a $U$.

Definição 12. Para cada $U \in \mathfrak{g}$, a regra suave

$$
U^{*}: p \mapsto U^{*}(p):=\left.\frac{d}{d t}\right|_{t=0} \exp (t U) \cdot p
$$

define campos globais em $M$, chamados de campos de ação.

Observação. Se $(M, g)$ é uma G-variedade riemanniana, então os campos de ação são campos de Killing.

Sejam $\mathfrak{g}, \mathfrak{g}_{p}$, as álgebras de Lie de $G$ e $G_{p}, p \in M$, respectivamente. Fixada uma métrica bi-invariante $Q$ em $G$, podemos decompor $\mathfrak{g}$ da seguinte maneira

$$
\mathfrak{g}=\mathfrak{g}_{p} \oplus \mathfrak{m}_{p}, \mathfrak{m}_{p}=\left(\mathfrak{g}_{p}\right)^{\perp_{Q}}
$$

A aplicação $\rho:(g, p) \mapsto \rho(g) p$ induz um difeomorfismo entre $G p \cong G / G_{p}$, de onde segue que o 
espaço tangente à órbita $G p$ é isomorfo a

$$
\left.\frac{d}{d t}\right|_{t=0} \exp \left(\mathfrak{m}_{p}\right) \cdot p .
$$

Definição 13. Uma fibração principal consiste em uma $G$-variedade suave $P$ cuja ação por $G$ é livre.

Proposição 3.1.3. Para uma fibração princial vale que,

1. $G p \cong G$ para cada $p$ (via difeomorfismos),

2. O espaço das órbitas $P / G$ admite uma única estrutura suave para a qual a aplicação $\pi: P \rightarrow$ $P / G$ é uma submersão.

Demonstração. Veja [AB15, Theorem 3.34, p. 60]

Convenção. Para o que segue denotaremos $P / G:=B$.

O objetivo das próximas linhas consiste em explicitar a dualidade entre fibrados principais topológicos e fibrados associados.

Definição 14 (Fibrado Principal). Seja $\pi: P \rightarrow B$ uma fibração principal. Assuma que exista pelo menos uma coleção de trivializações locais para $\pi: P \rightarrow B$. Isto é, que exista uma coleção

$$
\left\{\left(\phi_{i}, U_{i}\right): \phi_{i}: \pi^{-1}\left(U_{i}\right) \rightarrow U_{i} \times G\right\}
$$

tal que

1. $\left\{U_{i}\right\}$ são abertos em $B$,

2. $\phi_{i}$ são difeomorfismos tais que, se $U_{i} \cap U_{j} \neq \emptyset$, então a aplicação

$$
\varphi_{i j}: U_{i} \cap U_{j} \rightarrow G
$$

caracterizada por

$$
\phi_{i} \circ \phi_{j}^{-1}:(p, g) \mapsto\left(p, \varphi_{i j}(p) g\right), \forall p \in U_{i} \cap U_{j}, g \in G
$$

é contínua e satisfaz a seguinte condição de cociclo:

$$
\varphi_{i j} \varphi_{j k}(p)=\varphi_{i k}(p)
$$

Nesse caso, a submersão $\pi: G \hookrightarrow P \rightarrow B$ munida de uma coleção de trivializações locais (maximal) será denominada fibrado principal com espaço total $P$, base $B$ e fibra $G$.

Observação. O termo maximal empregado na definição anterior é análogo ao empregado no contexto de atlas para variedades diferenciáveis.

Mais geralmente, podemos considerar fibrados com fibras arbitrárias:

Definição 15 (Fibrado topológico). Um fibrado topológico com grupo de estrutura $G$, base $B$, espaço total $M$ e fibra $F$ consiste em uma submersão $\bar{\pi}: F \hookrightarrow M \rightarrow B$, onde $F$ é uma $G$-variedade à esquerda, munida de uma coleção (maximal)

$$
\left\{\left(U_{i}, \phi_{i}: \pi^{-1}\left(U_{i}\right) \rightarrow U_{i} \times F\right)\right\}
$$

tal que

1. $\left\{U_{i}\right\}$ são abertos em $B$, 
2. $\phi_{i}$ são difeomorfismos tais que, se $U_{i} \cap U_{j} \neq \emptyset$, então s aplicação

$$
\varphi_{i j}: U_{i} \cap U_{i} \rightarrow G
$$

caracterizada por

$$
\phi_{i} \circ \phi_{j}^{-1}(p, f):=\left(p, \varphi_{i j}(p) f\right), \forall p \in U_{i} \cap U_{i}, f \in F
$$

é contínua e satisfaz a seguinte condição de cociclo:

$$
\varphi_{i j} \varphi_{j k}(p)=\varphi_{i k}(p)
$$

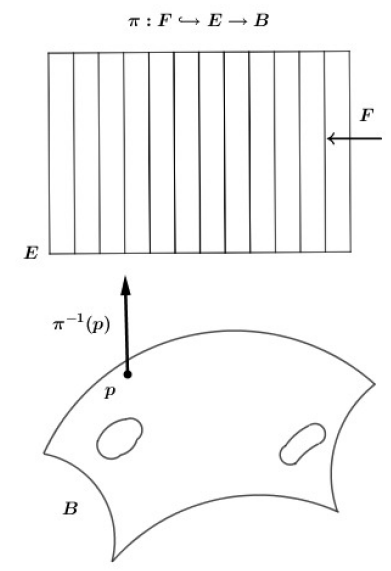

Figura 3.1: Representação gráfica de um fibrado topológico.

Definição 16. Uma coleção $\left\{\varphi_{i j}\right\}$ como nas Definições 14 e 15 é chamada de coleção de funções de transição associadas a $\pi, \bar{\pi}$, respectivamente.

Exemplo (Fibrado associado). Dado um G-fibrado principal (à direita) $\pi: P \rightarrow B$ e uma $G$ variedade (à esquerda) $F$, a variedade produto $P \times F$ admite uma $G$-ação efetiva e livre (à direita) definida por

$$
(p, f) g:=\left(p g, g^{-1} f\right) \in P \times F, g \in G .
$$

$O$ espaço de órbitas associado a (3.14) é usualmente denotado por $P \times_{G} F$ e é realizado como espaço total de um fibrado topológico com fibra $F$ e base B (veja [KN63, Chapter 5, p. 54-55]).

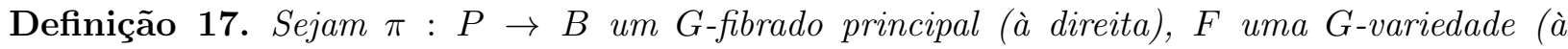
esquerda), e $P \times F$ a G-variedade segundo a ação (3.14). A submersão

$$
\bar{\pi}: F \hookrightarrow P \times_{G} F \rightarrow B
$$

é denominada fibrado associado a $P$ com fibra $F$ e grupo de estrutura $G$.

Exemplo (Fibrados topológicos como fibrados associados). De maneira recíproca, seja $\bar{\pi}: F \hookrightarrow$ $M \rightarrow B$ um fibrado topológico com grupo de estrutura $G$ e funções de transição $\Phi:=\left\{\varphi_{i j}\right\}$. Podemos usar o conjunto $\Phi$ para obter um $G$-fibrado principal (à direita) $P \rightarrow B$ (veja [KN63, Proposition 5.2, p. 52]). Se munirmos $P \times F$ com a ação efetiva e livre definida por

$$
(p, f) g:=\left(p g, g^{-1} f\right) \in P \times F, g \in G
$$

obtemos como espaço de órbitas associado à ação (3.15) a variedade $M$.

Concluímos portanto que podemos associar $G$-variedades a $G$-fibrados principais, originando um fibrado topológico. Reciprocamente, um fibrado topológico pode ser realizado via uma construção de fibrado associado. 
Seja $\pi: P \rightarrow B$ um fibrado principal. Assuma que $P$ esteja munida de uma métrica riemanniana $G$-invariante $g$. Seja $F$ uma $G$-variedade (à esquerda) munida de uma métrica $G$-invariante $g_{F}$. A projeção no quociente

$$
\text { pr : }\left(P \times F, g \times G_{p}\right) \rightarrow P \times_{G} F,
$$

induz uma métrica riemanniana $h$ em $P \times{ }_{G} F$ para qual pr é uma submersão riemanniana. De fato, se $\mathcal{L}_{\text {pr }}$ denota o levantamento horizontal por pr, lembrando que esse define um isomorfismo ponto a ponto entre $\mathcal{H}_{(p, f)} \subset T_{(p, f)} P \times F$ e $T_{\operatorname{pr}(p, f)} P \times_{G} F$, dado um ponto $[(p, f)] \in P \times_{G} F$, podemos definir $h$ segundo a relação

$$
h_{[(p, f)]}(\cdot, \cdot):=g \times g_{F(p, f)}\left(\mathcal{L}_{\mathrm{p} r} \cdot, \mathcal{L}_{\mathrm{p} r} \cdot\right) .
$$

Note que essa fica bem definida independente da escolha do representante em $[(p, f)]$. Precisamente, se $(p, f)$ e $\left(p^{\prime}, f^{\prime}\right)$ definem o mesmo elemento em $[(p, f)]$, então existe $g \in G$ tal que $p^{\prime}=p g, f^{\prime}=$ $g^{-1} f$. Como $g$ e $g_{F}$ são $G$-invariantes temos

$$
g \times g_{F\left(p^{\prime}, f^{\prime}\right)}=g \times g_{F\left(p g, g^{-1} f\right)}=g \times g_{F(p, f)} .
$$

Definição 18. Um fibrado principal $\pi: P \rightarrow B$ é dito fibrado principal riemanniano se o espaço total $P$ está munido de uma métrica riemanniana $G$-invariante cuja base $B$ está munida com a métrica de submersão.

Uma vez feita a Definição 18, uma pergunta natural se dá sobre a existência de métricas invariantes. Quando $G$ é um Grupo de Lie compacto e $\pi: P \rightarrow B$ é um $G$-fibrado principal, então sempre existe uma métrica $G$-invariante. Esse é um caso particular do Teorema 3.65 em [AB15, p. 70], tal que em sua demonstração é utilizada a técnica de média, definida a seguir.

Definição 19 (Média). Dado un tensor $T$ do tipo $(r, s)$ numa variedade riemanniana $(M, g)$ munida de uma ação isométrica por um Grupo de Lie compacto $G$ munido de uma métrica bi-invariante $Q$, o média de $T$ consiste no tensor $G$-invariante $T_{G}$ definido por

$$
\left(T_{G}\right)_{p}\left(X_{1}, \ldots, X_{r}, \omega^{1}, \ldots, \omega^{s}\right):=\int_{G} T_{r p}\left(r X_{1}, \ldots, r X_{r}, r \omega^{1}, \ldots, r \omega^{s}\right) d r, r \in G
$$

$\forall X_{i} \in T_{p} M, \omega^{j} \in T_{p}^{*} M, \forall i, j$, onde $d r$ denota a forma de volume em $G$ com respeito à métrica $Q$ e $r X_{i}$, r $\omega^{j}$ denota a imagem das aplicações induzidas por $\rho(r)$ em $T_{p} M$ e $T_{p}^{*} M$, respectivamente.

Proposição 3.1.4. Seja $\pi_{P}:\left(P, g_{P}\right) \rightarrow(B, h)$ um fibrado principal riemanniano. Dada uma $G$ variedade riemanniana $\left(F, g_{F}\right)$, existe uma única métrica riemanniana em $P \times{ }_{G} F$ para qual

$$
\pi: P \times_{G} F \rightarrow B
$$

é uma submersão riemanniana com fibras totalmente geodésicas e isométricas a $F$, e cuja distribuição horizontal é dada por $\widetilde{\mathcal{H}}:=d \operatorname{pr}(\mathcal{H} \times\{0\})$, onde pr é como na equação (3.16).

Demonstração. Veja [GW09, Proposition 2.7.1, p. 97]

O seguinte Teorema, de forma surpreendente, não é tão popular entre muitos pesquisadores da área, embora conste na literatura e seja fundamental para esta tese. Na seção seguinte, após introduzidas algumas deformações métricas, apresentaremos uma demonstração original obtida justamente por desconhecimento (inicial) do resultado. Outra prova pode ser vista em [GW09, Theorem 2.7.3, p. 100].

Teorema 3.1.5. Sejam $\left(B, g_{B}\right)$ e $\left(F, g_{F}\right)$ variedades riemannianas compactas e conexas com curvatura de Ricci positiva. Seja $\pi: F \hookrightarrow E \rightarrow B$ um fibrado topológico com grupo de estrutura $G$ compacto e conexo. Assuma que a métrica $g_{F}$ seja $G$-invariante. Então E admite uma métrica com curvatura de Ricci positiva. 


\subsubsection{Folheações riemannianas singulares e ações isométricas}

Nesta subseção introduziremos mais algumas propriedades de ações isométricas. Para a apresentação, traçaremos o paralelo com a subseção anterior, as introduzindo como exemplos de folheações singulares riemannianas, para as quais obtemos alguns resultados originais, a serem expostos na seção 3.3 .

Definição 20 (Folheações singulares riemannianas). Seja $(M, g)$ uma variedade riemanniana completa. Uma folheação singular riemanniana em $(M, g)$ consiste em uma decomposição de $M$ em subvariedades (conexas) imersas $\mathcal{F}=\left\{L_{p}\right\}_{p \in M}$, chamadas de folhas, tal que

1. Para cada $p \in M$ as imersões $L_{p} \hookrightarrow M$ são injetoras, em particular, dados $p, q \in M, L_{p} \cap L_{q}=$ $\emptyset$

2. Existe uma coleção de campos de vetores suaves $\left\{X_{\alpha}\right\}_{\alpha} \subset \Gamma(T M)$ que geram (como espaço vetorial) $T_{p} L_{p}, \forall p \in M$,

3. Para todo ponto $p \in M$ e toda geodésica $\gamma:(-\epsilon, \epsilon) \rightarrow M$ tal que $\gamma^{\prime}(0) \perp L_{p}$, vale que $\gamma^{\prime}(s) \perp L_{\gamma(s)} \forall s \in(-\epsilon, \epsilon)$. Em particular, as folhas de $\mathcal{F}$ são localmente equidistantes.

Algumas diferenças entre folheações riemannianas singulares para folheações riemannianas regulares induzidas por uma submersão riemanniana podem ser imediatamente notadas:

1. Na Definição 20, as folhas são assumidamente conexas. Mesmo que $\pi:(M, g) \rightarrow(N, h)$ seja uma sumersão riemanniana entre variedades conexas, não necessariamente suas folhas o são. No entanto, as componentes conexas do conjunto das fibras de $\pi$ formam uma folheação singular riemanniana.

2. As folhas podem ter dimensões diferentes. Entretanto, pode-se mostrar que a função $p \mapsto$ $\operatorname{dim} L_{p}$ é semi-contínua inferiormente. Assim, existe um aberto onde a dimensão das folhas é maximal.

3. As folhas de uma submersão, ou de uma folheação regular riemanniana, são sempre variedades fechadas. Isso não é necessariamente verdade para folheações singulares riemannianas. Existem exemplos dessas com folhas densas, como o fluxo irracional no toro plano, embora esse não seja um exemplo de folheação riemanniana.

Definição 21. Seja $(M, g)$ uma variedade riemanniana completa com uma folheação singular riemanniana $\mathcal{F}$. Dizemos que uma folha $L \in \mathcal{F}$ é regular se tiver dimensão maximal no conjunto de dimensões das folhas de $\mathcal{F}$. Caso contrário, dizemos que $L$ é singular.

Seja $(M, g)$ uma $G$-variedade compacta e conexa. Assuma que $G$ seja compacto e conexo. Analogamente ao caso de folheações regulares, definimos:

Definição 22. Sejam $p \in M$ e Gp uma órbita em $M$.

1. O espaço tangente $T_{p} G p$ é denominado espaço vertical em $p$, sendo denotado por $\mathcal{V}_{p}$,

2. O complemento ortogonal $\mathcal{H}_{p}:=\left(\mathcal{V}_{p}\right)^{\perp_{g}}$ é denominado espaço horizontal em $p$.

Note que a união dos espaços verticais não precisa definir uma distribuição em $T M$, já que podem existir pontos $p, q$ tais que $\mathcal{V}_{p}$ e $\mathcal{V}_{q}$ têm dimensões diferentes. Por outro lado, ações isométricas com órbitas singualres são exemplos de folheações singulares riemannianas.

Exemplo (Ações isométricas como folheações singulares riemannianas). Verifiquemos que as ações isométricas são exemplos de folheações singulares riemannianas.

A Proposição 3.1 .3 garante que as órbitas são subvariedades mergulhadas. Ademais, os campos de ação $\left\{U^{*}, U \in \mathfrak{m}_{p}\right\}$ (Definição 12) formam um subconjunto de $\Gamma(T M)$ que gera $\mathcal{V}_{p}$ para cada $p \in M$. 
Por fim, sejam $p \in M$ e $X \in \mathcal{H}_{p}$. Considere a geodésica $\gamma(s)=\exp _{p}(s X), s \in(-\epsilon, \epsilon)$, para algum $\epsilon>0$. Para evitar casos triviais, assuma que para cada $s \in(-\epsilon, \epsilon), \gamma(s)$ não seja um ponto fixo segundo a ação por $G$. Seja $U^{*}(s) \in \mathcal{V}_{\gamma(s)}$. Como a ação de $G$ em $M$ é por isometrias, o campo de ação $U^{*}(s)$ é um campo de Killing. Mas Campos de Killing são também Campos de Jacobi ao longo de geodésicas, e $U^{*}(p)$ é perpendicular a $\gamma^{\prime}(0)=X$. Daí, necessariamente $U^{*}(s)$ é perpendicular a $\gamma(s) \forall s \in(-\epsilon, \epsilon)$, o que conclui o afirmado.

\section{Slices e vizinhanças tubulares}

As motivações desta subseção consistem em introduzir o Teorema de Slice 3.1.10 e fornecer os primeiros passos para uma descrição precisa do conceito de estratificação. De fato, nada impede, a princípio, que existam pontos $p, q \in M$ tais que $G_{p}$ não seja conjugado a $G_{q}$. Nesse caso, as órbitas $G p$ e $G q$ não seriam difeomorfas, e poderiam ter dimensões diferentes. Nas próximas linhas caracterizemos os estratos e pontos singulares associados a uma ação isométrica, destacando a existência um aberto denso e convexo $M^{r e g}$ em $M$ no qual para cada $p, q \in M^{r e g}, \exists g \in G$ : $g G_{p} g^{-1} g \cong G_{q}$.

Seja $M$ uma $G$-variedade (à esquerda). No que segue, lembre-se que a ação por $G$ pode ser pensada como um mapa $\rho: G \times M \rightarrow M$. Portanto, dado $p \in M$, denotaremos $\rho(p): G \rightarrow M$.

Definição 23 (Slice). Um slice em $p_{0} \in M$ consiste em uma subvariedade mergulhada $S_{p_{0}} \ni p_{0}$ tal que:

1. $T_{p_{0}} M=d \rho\left(p_{0}\right)(\mathfrak{g}) \oplus T_{p_{0}} S_{p_{0}}$ e para todo $p \in S_{p_{0}}$ tem-se $T_{p} M=d \rho(p)(\mathfrak{g})+T_{p} S_{p_{0}} ;$

2. $S_{p_{0}}$ é invariante pela ação de $G_{p_{0}}$. Precisamente, para todos $p \in M, g \in G_{p_{0}}$, tem-se gp $\in S_{p_{0}}$;

3. Se $p \in S_{p_{0}}$ e $g \in G$ são tais que $g p \in S_{p_{0}}$, então $g \in G_{p_{0}}$.

Teorema 3.1.6 (Existência de Slices). Seja $M$ uma $G$-variedade compacta com $G$ também compacto. Então para qualquer $p_{0} \in M$ existe um slice $S_{p_{0}}$ em $p_{0}$.

Observação. Enunciamos o Teorema 3.1 .6 com hipóteses muito fortes, mas suficientes para essa tese. O resultado vale de forma mais simples: existe um slice em cada ponto onde a ação por $G$ é própria.

Esboçaremos a demonstração do Teorema 3.1.6 por acreditar que durante a prova ficam bem explícitos os candidatos a slices.

Esboço da demonstração do Teorema 3.1.6. Assuma que $(M, g)$ seja uma variedade riemanniana compacta e conexa munida de uma ação isométrica por um grupo de Lie compacto e conexo $G$. Fixado $p_{0} \in M$, seja $G_{p_{0}}$ o subgrupo de isotropia de $G$ em $p_{0}$. Como $g$ é invariante pela ação de $G$ e $G_{p_{0}}$ é subgrupo de Lie de $G$, então $g$ é invariante pela ação de $G_{p_{0}}$. Note que para cada $g \in G_{p_{0}}$ a diferencial de $\rho\left(g, p_{0}\right) \equiv \rho_{g}\left(p_{0}\right)$ define uma aplicação linear de $T_{p_{0}} M$ em $T_{p_{0}} M, d\left(\rho_{g}\right)_{p_{0}}: T_{p_{0}} M \rightarrow$ $T_{p_{0}} M$. Mais ainda, essa aplicação deixa invariante o subespaço tangente à órbita de $G$ por $p_{0}$, isto é, $T_{p_{0}} G\left(p_{0}\right)$. Como a ação por $G_{p_{0}}$ é via isometrias, então o espaço horizontal $\mathcal{H}_{p_{0}}$ em $p_{0}$, isto é, o o subespaço obtido como complemento ortogonal de $T_{p_{0}} G\left(p_{0}\right)$ em $T_{p_{0}} M$ também é invariante.

Assim, se $B_{\epsilon}(0)$ é uma bola de raio $\epsilon$ com centro na origem de $\mathcal{H}_{p}$, um candidato a slice consiste em $S_{p_{0}}:=\exp \left(B_{\epsilon}(0)\right)$. Como $\mathcal{H}_{p}$ é $G_{p_{0}}$-invariante e isometrias mapeiam geodésicas em geodésicas, segue portanto que $S_{p_{0}}$ é invariante pela ação de $G_{p_{0}}$. Esse fato verifica o item 2 na definição de slice, enquanto o item 1 é verificado imediatamente considerando a maneira como o slice foi construído. É apenas para o ponto 3 que o fato da ação ser própria em $p_{0}$ é utilizado, veja [AB15, Theorem 3.49 , p. 65].

Exemplo. Considere $M=\mathbb{R}^{3} \equiv \mathbb{C} \times \mathbb{R}$ munida da ação à esquerda por $G=U(1) \times \mathbb{R}$ definida por

$$
\left(e^{i \theta}, t\right) \cdot\left(x_{1}, x_{2}, x_{3}\right) \equiv\left(z, x_{3}\right):=\left(e^{i \theta} z, x_{3}+t\right), z=x_{1}+i x_{2}, \theta, t \in \mathbb{R} .
$$


Fixe $\vec{x}=\left(x_{1}, x_{2}, x_{3}\right)$ tal que $|z|^{2}=r^{2}, r>0$. Note que a órbita de $\vec{x}$ por $G$ é dada por um cilindro reto com eixo $\left(0+0 i, x_{3}\right)$. Mais ainda, a isotropia em $\vec{x}$ é dada por $G_{\vec{x}}=\{(1+0 i, 0)\}$. Se $r=0$, i.e, $\vec{x}=\left(0+0 i, x_{3}\right)$ então a órbita de $\vec{x}$ por $G$ consiste no eixo $\left(0+0 i, x_{3}\right)$. Nesse caso, $G_{\vec{x}}=U(1)$.

No primeiro caso $(r>0)$, um slice em $\vec{x}$ consiste na reta $r:\left(0+0 i, x_{3}\right)+t(z, 0),|t-1|<\epsilon$. No caso em que $r=0$ um slice consiste em um disco $\left\{\left(\widetilde{z}, x_{3}\right):|\widetilde{z}|^{2}<\epsilon\right\}$.

Seja $M$ uma $G$-variade (à esquerda) compacta onde $G$ é também compacto. Dado $p_{0} \in M$ seja $S_{p_{0}}$ um slice em $p_{0}$.

Definição 24 (Vizinhança tubular de uma órbita). Uma vizinhança tubular da órbia Gp $p_{0}$ consiste em

$$
\operatorname{Tub}\left(G p_{0}\right):=G S_{p_{0}} .
$$

O próximo Teorema fornece uma descrição de vizinhanças tubulares como fibrados associados. Para tanto, lançamos mão da seguinte definição.

Definição 25. Sejam $G$ um grupo de Lie e $M, N$ G-variedades (à esquerda). Uma função suave $f: M \rightarrow N$ é dita $G$-equivariante se

$$
f(g p)=g f(p), \forall p \in M, \forall g \in G .
$$

Teorema 3.1.7 (Caracterização de vizinhanças tubulares). Seja $M$ uma G-variade (à esquerda) onde $M$ e $G$ são compactos. Então para cada $p_{0} \in M$ existe um difeomorfismo $G$-equivariante entre $\operatorname{Tub}\left(G p_{0}\right)=G S_{p_{0}}$ e o espaço total do fibrado

$$
S_{p_{0}} \rightarrow G \times_{G_{p_{0}}} S_{p_{0}} \rightarrow G / G_{p_{0}} .
$$

Demonstração. Veja [AB15, Theorem 3.57, p. 68].

\section{Órbitas principais, tipos de órbita e estratificação}

Quando introduzimos o conceito de folheações singulares riemannianas, mencionamos sem demonstração que existe um aberto denso onde a dimensão das folhas é maximal. O objetivo das próximas linhas reside em entender essa manifestação no caso de ações isométricas (onde a ação não é livre). Resultados relacionados sobre diferentes tipos de órbita serão mencionados e explicados em algum detalhe, uma vez que são fundamentais para os problemas abordados na presente tese.

Convenção. Durante todo o restante dessa seção assumiremos que $M$ seja uma G-variedade (à esquerda); $M$ e $G$ são compactos, e por simplicidade, $M$ e $G$ são também conexos.

Definição 26. Uma órbita $G p, p \in M$ é dita principal se existir uma vizinhança $V \ni p$ tal que para qualquer $q \in V$ existe $g_{q} \in G$ para o qual $G_{p} \subset G_{g q}$.

Em palavras, órbitas principais são tais que seus pontos possuem a menor isotropia possível.

Proposição 3.1.8. São equivalentes:

1. Gp, $p \in M$, é uma órbita principal;

2. Se $S_{p}$ é um slice em $p$, então $G_{p}=G_{q}$ para todo $q \in S_{p}$.

Assuma que $M$ esteja munida de uma métrica riemanniana $g$ e que a ação de $G$ em $M$ seja por isometrias. Como vimos, as componentes conexas de das órbitas por $G$ formam uma folheação singular riemanniana. Em particular, se uma geodésica $\gamma(s)$ é ortogonal a uma órbita, essa é ortogonal a toda órbita que encontra. Em particular, essa propriedade diz que as órbitas de uma ação isométrica são localmente equidistantes.

Precisamente, se um segmento geodésico $\gamma:[0,1] \rightarrow M$ minimiza a distância entre $G \gamma(0)$ e $\gamma(1)$, então $\gamma$ é ortogonal a $G \gamma(0)$. Logo, de acordo com a discussão supracitada, $\gamma$ deve ser uma geodésica 
horizontal. Dado que para todo $g \in M$ temos que $\rho_{g}: M \rightarrow M$ é uma isometria, a curva $\rho_{g} \circ \gamma$ (ou simplesmente $g \gamma$ ) é um segmento geodésico que liga $G \gamma(0)$ a $g \gamma(1) \in G \gamma(1)$. Em particular, temos igualdade entre os comprimentos $\ell(\gamma)=\ell(g \gamma)$, de onde concluímos que duas órbitas são localmente equidistantes. Em particular, fica bem definida uma distância orbital que torna $M / G$ um espaço métrico.

Teorema 3.1.9 (Teorema das órbitas principais). Denote por $M^{\text {reg }}$ o subconjunto de $M$ que consiste em pontos contidos em órbitas principais. Então vale que:

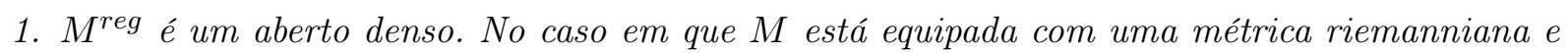
a ação por $G$ é via isometrias, temos ainda que $M^{\text {reg }}$ é convexo;

2. O aberto $M^{\text {reg }} / G \subset M / G$ é uma variedade suave;

3. Para quaisquer dois pontos $p, q \in M$, as isotropias $G_{p}$ e $G_{q}$ são conjugadas. Em particular, duas órbitas principais são difeomorfas.

Demonstração. Veja [AB15, Theorem 3.82, p. 75]

Órbitas principais são apenas um dos possíveis tipos de órbitas. No que segue discutiremos as possibilidades para essas em uma variedade compacta com ação por um grupo compacto. Nesse contexto, o número de tipos de órbitas é sempre finito.

Definição 27 (Tipos de órbita). Sejam $p, q \in M$.

1. A órbita Gp é dita ser de maior tipo que Gq se existir um mapa $G$-equivariante $\varphi: G p \rightarrow G q$, ou equivalentemente, se existir $g \in G$ tal que $G_{p} \subset G_{g q}$;

2. As órbitas Gp e Gq possuem o mesmo tipo se existir um difeomorfismo $G$-equivariante $\varphi$ : $G p \rightarrow G q$, ou equivalentemente, se existir $g \in G$ tal que $G_{p}=G_{g q}$;

3. A órbita Gp é dita regular se tiver mesma dimensão que uma órbita principal;

4. Uma órbita principal que não seja regular é dita excepcional;

5. Uma órbita não-regular é dita singular.

Como a ação de $G$ em cada órbita é transitiva, usaremos a nomenclatura na definição de tipos de órbita também para pontos. Mais ainda, separar órbitas em tipos define relações de equivalência. Em particular, o Teorema 3.1.9 garante que existe um único tipo de órbita principal.

Teorema 3.1.10 (Teorema de Slice). Para cada $p \in M$ existe um slice $S_{p}$ tal que a vizinhança tubular $\operatorname{Tub}(G p)$ contém apenas um número finito de tipos de órbitas. Em particular, se $M$ é compacta existe um número finito de slices.

Demonstração. Veja [AB15, Teorema 3.91, p. 78].

Definição 28 (Tipo local de órbitas). Duas órbitas Gp e Gq possuem o mesmo tipo local de órbita se existe um difeomorfismo equivariante $\varphi: \operatorname{Tub}(G p) \rightarrow \operatorname{Tub}(G q)$.

Note portanto que introduzimos duas relações de equivaliencia, a saber, tipo de órbita e tipo local de órbita. Simbolizaremos essas relações por $\sim$ e $\approx$, respectivamente. Observamos que a relação $\approx$ é mais restritiva. Assim, as classes de equivalência segundo a relação $\sim$ podem ser particionadas com respeito às classes de equivalência segundo $\approx$. Denotamos,

$$
M_{p}^{\sim}=\{q \in M: q \sim p\}, \quad M_{p}^{\approx}=\{q \in M: q \approx p\},
$$

e os denominamos tipo de órbita e tipo local de órbita, respectivamente. Segue que um tipo de órbita é uma união de tipo de locais de órbitas. Pode-se mostrar que $p \approx q$ se, e somente se, $p \sim q$ e as 
representações de isotropia $G_{p}$ e $G_{q}$ em $T_{p} M$ e $T_{q} M$, respectivamente, descem para representações equivalente sem $T_{p} M / T_{p} G p$ e $T_{q} M / T_{q} G q$, ou seja, esses espaços são linearmente equivariantemente isomorfos.

O próximo resultado permitirá definir precisamente o conceito de estratificação. Um esboço da demonstração pode ser visto em [AB15, Theorem 3.95, p. 80$]$.

Teorema 3.1.11 (Estrutura de tipos locais de órbitas). Cada tipo de órbita local $M_{p}^{\approx}, p \in M$, é a união de subvariedades $G$-invariantes, mergulhadas, e de mesma dimensão. Mais ainda, a restrição do mapa quociente $\pi: M \rightarrow M / G$ a $M_{\tilde{p}}$ determina um fibrado

$$
G / G_{p} \hookrightarrow M_{p}^{\approx} \rightarrow M^{\approx} / G,
$$

com grupo de estrutura $N\left(G_{p}\right) / G_{p}$, onde $N\left(G_{p}\right)$ denota o normalizador de $G_{p}$ em $G$. Em particular, os subconjuntos de $M \approx / G$ que possuam o mesmo tipo local de órbita são subvariedades suaves em $M / G$.

Definição 29 (Estratificação). Uma estratificação de $M$ consiste em uma partição de $M$ em subvariedades mergulhadas $\left\{M_{i}\right\}_{i \in I}$, denominadas estrato, tais que:

1. Essa partição é localmente finita. Ou seja, cada subconjunto compacto de $M$ intercepta no máximo um número finito de elementos do estrato;

2. Para cada $i \in I$ existe um subconjunto $I_{i} \subset I \backslash\{i\}$ tal que o fecho de $M_{i}$ admite a decomposição $\bar{M}_{i}=M_{i} \cup \bigcup_{j \in I_{i}} M_{j}$;

3. $\operatorname{dim} M_{j}<\operatorname{dim} M_{i}, \forall j \in I_{i}$.

Teorema 3.1.12 (Teorema de Estratificação). A partição de $M$ via as componentes conexas dos tipos de órbita $M_{p}^{\sim}, p \in M$, determina uma estratificação de $M$.

Demonstração. Veja [AB15, Theorem 3.102].

Como último resultado, mostramos que as hipóteses de órbitas com grupo fundamental finito, como no Teorema $\mathrm{C}$ e muitos outros nessa tese, fica completamente determinado por essa condição em uma órbita principal.

Teorema 3.1.13 (Sobre o grupo fundamental dos tipos de órbita). Se uma órbita principal possuir grupo fundamental finito, então o mesmo vale para todas as outras órbitas (de qualquer tipo).

Demonstração. De fato, seja $H \subset G$ um subgrupo de isotropia principal de acordo com a ação por $G$ em $M$, ou seja, $G / H$ é uma órbita princial. Por hipótese tem-se que $\pi_{1}(G / H)$ é finito. Como vimos, a menos de conjugação podemos assumir que $H \subset G_{p}$. A sequência longa de homotopia de $G_{p} / H \hookrightarrow G / H \rightarrow G / G_{p}$ fornece

$$
\cdots \rightarrow \pi_{1}(G / H) \rightarrow \pi_{1}\left(G / G_{p}\right) \rightarrow \pi_{0}\left(G_{p} / H\right) \rightarrow \cdots
$$

Portanto, $\pi_{1}\left(G / G_{p}\right)$ é finito se, e somente se, $G_{p} / H$ possuir um número finito de componentes conexas. Entretanto, como $G_{p}$ é uma variedade compacta e componentes conexas em variedades são abertas e fechadas, segue que $\pi_{1}\left(G / G_{p}\right)$ é finito.

\subsection{Deformações métricas clássicas}

A presente seção tem como objetivo introduzir algumas deformações métricas clássicas para as quais obtivemos alguns resultados. A referência principal é o livro [GW09]. Destacamos que todas as fórmulas de curvatura seccional apresentadas nesta subseção foram extraídas dessa referência, a menos de erratas. 
Seja $\pi:(M, g) \rightarrow B$ uma submersão riemanniana. Podemos obter outras submersões a partir de $\pi$ ao introduzir deformações métricas que só alteram a métrica $g$ nas direções verticais. Especificamente, seja $\phi: M \rightarrow \mathbb{R}$ uma função suave. Podemos definir uma nova métrica $\widetilde{g}$ em $M$ da seguinte maneira

$$
\widetilde{g}(E, F):=g\left(E^{\mathcal{H}}, F^{\mathcal{H}}\right)+e^{2 \phi} g\left(E^{\mathcal{V}}, F^{\mathcal{V}}\right), \forall E, F \in T_{p} M, \forall p \in M .
$$

Como a nova métrica não altera as direções horizontais, $\pi:(M, \widetilde{g}) \rightarrow B$ ainda é uma submersão riemanniana. Denote por $\widetilde{\nabla}, \widetilde{R}$ conexões de Levi-Civita e tensor de Riemann associados a $\widetilde{g}$.

Definição 30. A submersão riemanniana $\pi:(M, \widetilde{g}) \rightarrow B$ tal que $\phi$ é constante ao longo das fibras (ou, equivalentemente, $\nabla \phi$ é básico), será chamada de produto vertical geral.

Convenção. Nas proposições abaixo $|\cdot|$ representa a norma segundo a métrica $g$.

Proposição 3.2.1 (Curvaturas seccionais em produtos verticais gerais). Seja $(M, g)$ uma variedade riemanniana munida de uma folheação riemanniana regular. Seja $\widetilde{g}$ uma métrica de produto vertical geral por uma função $e^{2 \phi}, \phi \in C^{\infty}(M ; \mathbb{R})$. Fixe $p \in M$. Sejam $X, Y \in \mathcal{H}_{p}, V, V_{i} \in \mathcal{V}_{p}, i \in\{1,2\}$. Se $g\left(V_{1}, V_{2}\right)=0$, as seguintes fórmulas para curvatura seccional $\widetilde{K}$ de $\widetilde{g}$ são verdadeiras:

$$
\begin{gathered}
\widetilde{K}(X, Y)=\left(1-e^{2 \phi}\right) K_{B}(X, Y)+e^{2 \phi} K(X, Y), \\
\widetilde{K}\left(V_{1}, V_{2}\right)=\left(e^{2 \phi}-e^{4 \phi}\right) \\
K_{F}\left(V_{1}, V_{2}\right)+e^{4 \phi} K_{g}\left(V_{1}, V_{2}\right) \\
-e^{4 \phi}\left|V_{1}\right|^{2}\left|V_{2}\right|^{2}|\nabla \phi|^{2}+e^{4 \phi} d \phi\left(\sigma\left(V_{1}, V_{1}\right)\right)\left|V_{2}\right|^{2}+e^{4 \phi} d \phi\left(\sigma\left(V_{2}, V_{2}\right)\right)\left|V_{1}\right|^{2}, \\
\widetilde{K}(X, V)=K_{g}(X, V) e^{2 \phi}-e^{2 \phi}\left(1-e^{2 \phi}\right)\left|A_{X}^{*} V\right|^{2}- \\
\left\{\operatorname{Hess} \phi(X, X)+d \phi(X)^{2}\right\} e^{2 \phi}|V|^{2}+2 e^{2 \phi} d \phi(X) g\left(S_{X} V, V\right) .
\end{gathered}
$$

Demonstração. Veja [GW09, Section 2.1.3, p. 52]

\section{Produtos verticais (Warped products)}

Sejam $\left(B, g_{B}\right)$ e $\left(F, g_{F}\right)$ variedades riemannianas. Assuma que $\pi: M=B \times F \rightarrow B$ esteja munida da métrica produto. Considere uma função suave $\phi: B \rightarrow \mathbb{R}$. Então a métrica $\widetilde{g}:=g_{B} \times e^{2 \phi} g_{F}$ é um exemplo de produto vertical geral, ao qual denominaremos produto vertical. A variedade riemanniana $(M, \widetilde{g})$ é chamada de produto vertical de $B$ e $F$, sendo usualmente denotada por $B \times{ }_{e^{2 \phi}} F$.

Note que o tensor $A$ em produtos verticais se anula identicamente. Mais ainda, a segunda forma fundamental das fibras é dada por

$$
\widetilde{\sigma}\left(T_{1}, T_{2}\right)=-e^{2 \phi} g\left(T_{1}, T_{2}\right) \nabla \phi=-\widetilde{g}\left(T_{1}, T_{2}\right) .
$$

Em particular, em um produto vertical as fibras são subvariedades totalmente umbílicas e o campo de curvatura média $\widetilde{H}=-k \nabla \phi$ é básico. Em verdade, essas propriedades caracterizam produtos verticais:

Proposição 3.2.2. Seja $\pi:(M, g) \rightarrow B$ uma submersão riemanniana. Então, $\pi$ é localmente um produto vertical se, e somente se,

1. $[\mathcal{H}, \mathcal{H}] \subset \mathcal{H}$, consequentemente, o tensor de Gray-O'Neill se anula identicamente, isto é, $A \equiv 0$.

2. As fibras de $\pi$ são subvariedades totalmente umbilicas;

3. $\pi$ é isoparamétrica, ou seja, o vetor de curvatura média das fibras é básico. 
Demonstração. Veja [GW09, Proposition 2.2.1, p. 56].

As seguintes fórmulas são verdadeiras para uma métrica de produto vertical:

Proposição 3.2.3 (Fórmulas para curvatura seccional em produtos verticais). Considere um produto vertical $\pi:\left(M \times F, \widetilde{g}=g \times e^{2 \phi} g_{F}\right) \rightarrow M$ e seja $\widetilde{K}$ a curvatura seccional de $\widetilde{g}$. Fixe $(p, f) \in M \times F$ e tome $X, Y \in \mathcal{H}_{p}, V, V_{i} \in \mathcal{V}_{p}, i \in\{1,2\}$. Então

$$
\begin{aligned}
\widetilde{K}(X, Y) & =K_{M}(X, Y) \\
\widetilde{K}\left(V_{1}, V_{2}\right) & =e^{2 \phi}\left\{K_{F}\left(V_{1}, V_{2}\right)-e^{2 \phi}|\nabla \phi|^{2}\left(\left|V_{1}\right|^{2}\left|V_{2}\right|^{2}-\left\langle V_{1}, V_{2}\right\rangle\right)^{2}\right\} ; \\
\widetilde{K}(X, V) & =-e^{2 \phi}|V|^{2}\left(\langle\nabla \phi, X\rangle^{2}+\operatorname{Hess} \phi(X, X)\right),
\end{aligned}
$$

\section{Deformação canônica}

Outro caso particular de produto vertical geral consiste em assumir que $\phi(p)=t \in \mathbb{R}, \forall p \in M$. Nesse caso, a métrica $\widetilde{g}$ é dita uma deformação canônica de $g$. Para a próxima proposição, seja $\widetilde{g}=\left.g\right|_{\mathcal{B}}+\left.e^{2 t} g\right|_{\mathcal{V}}$.

Proposição 3.2.4 (Fórmulas para curvatura seccional de uma deformação canônica). Seja $\pi$ : $\left(F, g_{F}\right) \hookrightarrow(M, g) \rightarrow\left(B, g_{B}\right)$ uma submersão riemanniana com fibras totalmente geodésicas. Denote por $\widetilde{K}, K, K_{B}, K_{F}$ as curvatura seccionais não reduzidas de $\widetilde{g}, g, g_{B}, g_{F}$, respectivamente. Fixados $p \in M, X, Y, Z \in \mathcal{H}_{p}$, e $V, U \in \mathcal{V}_{p}$, as seguintes fórmulas são verdadeiras:

1. $\widetilde{K}(X, Y)=K_{B}\left(\pi_{*} X, \pi_{*} Y\right)\left(1-e^{2 t}\right)+e^{2 t} K(X, Y)$,

2. $\widetilde{K}(X, V)=e^{4 t}\left|A_{X}^{*} V\right|^{2}$,

3. $\widetilde{K}(U, V)=e^{2 t} K(U, V)$,

4. $\widetilde{R}(X, Y, Z, V)=e^{2 t} g\left(\left(\nabla_{X} A\right)_{Y} Z, V\right)$.

Agora estamos em condições de provar o Teorema 3.1.5.

Demonstração do Teorema 3.1.5. Recordando a discussão apresentada na seção 3.1 sobre fibrados associados, lembre-se que $E$ pode ser obtido como um fibrado associado $E=P \times_{G} F \rightarrow B$. Seja $g$ a única métrica riemanniana em $E$ cujas fibras totalmente geodésicas e isométricas a $F$. Tal métrica existe e é única de acordo com a Proposição 3.1.4.

Tome $p \in \mathcal{H}, V \in \mathcal{V},|X|_{\widetilde{g}}=|V|_{\widetilde{g}}=1$ e seja $\lambda \in \mathbb{R}$. Considere o polinômio $p(\lambda):=\widetilde{\operatorname{Ric}}(X+\lambda V)$. Tome uma base $g$-ortonormal $\left\{\widetilde{e}_{i}:=e_{i}\right\}_{i=1}^{k}$ para $\mathcal{H}$ e $\left\{\widetilde{e}_{j}:=e^{-t} e_{j}\right\}_{j=k+1}^{n}$ uma base $\widetilde{g}$-ortonormal para $\mathcal{V}$. Então,

$$
\widetilde{R}\left(X+\lambda V, \widetilde{e}_{r}, \widetilde{e}_{r}, X+\lambda V\right)=\widetilde{K}\left(X, \widetilde{e}_{r}\right)+\lambda^{2} \widetilde{K}\left(V, \widetilde{e}_{r}\right)+2 \lambda \widetilde{R}\left(X, \widetilde{e}_{r}, \widetilde{e}_{r}, V\right),
$$

$\forall r \in\{1, \ldots, n\}$

Somando em $i, j$, e usando as fórmulas fornecidas na Proposição 3.2.4, temos:

$$
\begin{aligned}
p(\lambda)=\left(1-e^{2 t}\right) \operatorname{Ric}_{B}(p) & +e^{2 t} \operatorname{Ric}^{\mathcal{H}}(p)+e^{2 t} \sum_{j=k+1}^{n}\left|A_{X}^{*} e_{j}\right|^{2} \\
& +\lambda^{2}\left\{e^{2 t} \sum_{i=1}^{k}\left|A_{e_{i}} V^{*}\right|^{2}+\operatorname{Ric}_{F}(V)\right\}+2 \lambda e^{2 t} \sum_{i=1}^{k} g\left(\left(\nabla_{e_{i}} A\right)_{X} e_{i}, V\right) .
\end{aligned}
$$


O discriminante $\Delta_{t}(X, V)$ é dado por:

$$
\begin{aligned}
& \frac{1}{4} \Delta_{t}(X, V)=e^{4 t}\left(\sum_{i=1}^{k} g\left(\left(\nabla_{e_{i}} A\right) X e_{i}, V\right)\right)^{2}- \\
& \left(\left(1-e^{2 t}\right) \operatorname{Ric}_{B}(p)+e^{2 t} \operatorname{Ric}^{\mathcal{H}}(p)+e^{2 t} \sum_{j=k+1}^{n}\left|A_{X}^{*} e_{j}\right|^{2}\right) \\
& \left(e^{2 t} \sum_{i=1}^{k}\left|A_{e_{i}} V^{*}\right|^{2}+\operatorname{Ric}_{F}(V)\right)
\end{aligned}
$$

Afirmamos que existe $t<0$ tal que para cada $X \in \mathcal{H}, V \in \mathcal{V},|X|=|V|=1$, vale que $\Delta_{t}(X, V)<0$. De fato, suponha por contradição que para cada $n \in \mathbb{N}$ existam $X_{n}, V_{n}$ como nas hipóteses e tais que $\Delta_{-n}\left(X_{n}, V_{n}\right) \geq 0$. Passando a uma subsequência se necessário e tomando $n \rightarrow \infty$ concluímos:

$$
-\operatorname{Ric}_{F}(V) \operatorname{Ric}_{B}(X) \geq 0
$$

onde $V_{n} \rightarrow V, X_{n} \rightarrow X$. Mas como por hipótese

$$
\operatorname{Ric}_{F}(V) \operatorname{Ric}_{B}(X)>0
$$

temos uma contradição, o que conclui a prova.
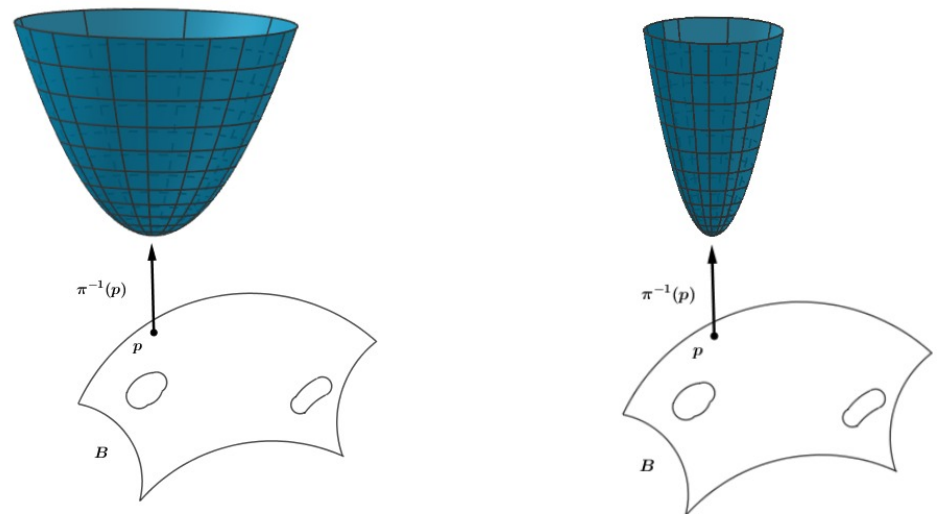

Figura 3.2: Na figura da esquerda vemos a disposição inicial de um fibrado topológico como nas hipóteses do Teorema 3.1.5. A figura da direita reflete a manifestação da deformação canônica nesse fibrado.

Um outro resultado que será importante para o restante do texto é o seguinte:

Teorema 3.2.5. Sejam $\left(M^{n}, g\right)$ e $\left(F^{k}, g_{F}\right)$ variedades riemannianas compactas e conexas tais que $g$ e $g_{F}$ possuem curvatura de Ricci positiva. Seja $\phi: M \rightarrow \mathbb{R}$ uma função suave. Então a variedade $M \times{ }_{e^{2 \phi}} F$ desenvolve curvatura de Ricci positiva após uma deformação canônica.

Demonstração. Fixe um ponto $(p, f) \in M \times F$. Vamos computar a curvatura de Ricci para métrica de produto vertical $\widetilde{g}_{t}=g \times e^{2 t \phi} g_{F}$. Tome uma base $g$-ortonormal $\left\{v_{i}\right\}_{i=1}^{n}$ para $T_{p} M$ uma base $g_{F}$-ortonormal $\left\{w_{j}\right\}_{j=1}^{k}$ para $T_{f} F$. Então $\left\{v_{i}\right\} \cup\left\{e^{-t \phi} w_{j}\right\}$ é uma base $\widetilde{g}_{t}$-ortonormal para $T_{(p, f)} \widetilde{M}$.

Denotemos por $\widetilde{R}_{t}(Z, W, W, Z):=\widetilde{K}_{t}(Z, W), \forall Z, W \in T_{(p, f)}^{1} \widetilde{M}$. Assuma que $|X|=|V|=1 \mathrm{e}$ seja $\lambda \in \mathbb{R}$. Assim,

$$
\widetilde{R}_{t}\left(X+\lambda V, v_{i}, v_{i}, X+\lambda V\right)=\widetilde{K}_{t}\left(X, v_{i}\right)+\lambda^{2} \widetilde{K}_{t}\left(V, v_{i}\right)
$$




$$
\widetilde{R}_{t}\left(X+\lambda V, e^{-t \phi} w_{j}, e^{-t \phi} w_{j}, X+\lambda V\right)=e^{-2 t \phi} \widetilde{K}_{t}\left(X, w_{j}\right)+\lambda^{2} e^{-2 t \phi} \widetilde{K}_{t}\left(V, w_{j}\right) .
$$

Logo, de acordo com a Proposição 3.2.3 temos

$$
\widetilde{\operatorname{Ric}_{t}}(X+\lambda V)=\operatorname{Ric}_{M}(X)+\lambda^{2} \sum_{i=1}^{n} \widetilde{K}_{t}\left(V, v_{i}\right)+e^{-2 t \phi} \sum_{j=1}^{k} \widetilde{K}_{t}\left(X, w_{j}\right)+\lambda^{2} e^{-2 t \phi} \operatorname{Ric}_{F}(V) .
$$

Considerando o polinômio

$$
p_{t}(\lambda)=\operatorname{Ric}_{M}(X)+\lambda^{2} \sum_{i=1}^{n} \widetilde{K}_{t}\left(V, v_{i}\right)+e^{-2 t \phi} \sum_{j=1}^{k} \widetilde{K}_{t}\left(X, w_{j}\right)+\lambda^{2} e^{-2 t \phi} \operatorname{Ric}_{F}(V),
$$

temos como discrimante

$$
\widetilde{\Delta}_{t}=-4\left(\sum_{i=1}^{n} \widetilde{K}_{t}\left(V, v_{i}\right)+e^{-2 t \phi} \operatorname{Ric}_{F}(V)\right)\left(\operatorname{Ric}_{M}(X)+e^{-2 t \phi} \sum_{j=1}^{k} \widetilde{K}_{t}\left(X, w_{j}\right)\right) .
$$

Como pela equação (3.23) temos

$$
\begin{aligned}
\widetilde{K}_{t}\left(V, v_{i}\right) & =-e^{2 t \phi}\left\{t^{2} d \phi\left(v_{i}\right)^{2}+t \operatorname{Hess} \phi\left(v_{i}, v_{i}\right)\right\}, \\
\widetilde{K}_{t}\left(X, w_{j}\right) & =-e^{2 t \phi}\left\{t^{2} d \phi(X)^{2}+t \operatorname{Hess} \phi(X, X)\right\},
\end{aligned}
$$

concluímos que

$$
\widetilde{\Delta}_{t}=4 e^{2 t \phi}\left(t^{2}|\nabla \phi|^{2}+t \Delta_{M} \phi-e^{-4 t \phi} \operatorname{Ric}_{F}(V)\right)\left(\operatorname{Ric}_{M}(X)-k\left(t^{2} d \phi(X)^{2}+t \operatorname{Hess}_{M} \phi(X, X)\right) .\right.
$$

Por argumento análogo ao utilizado na prova do Teorema 3.1.5, só que dessa vez fazendo $t \rightarrow 0$, conclui-se o afirmado.

\subsection{Um mínimo sobre fluxos geométricos: Solitons de Ricci e Fluxos de Curvatura Média}

A abordagem nesta seção será inteiramente sem coordenadas, e tem ambição de ser concisa. Para maiores detalhes indicamos [AH10, Chapter 3], [Top06], [Bre10].

Ao considerar mapas entre variedades riemannianas, é possível associar diversos "funcionais de energia", que atendem diferentes interesses geométricos e físicos.

Como veremos no Capítulo 7, tais funcionais surgem naturalmente em problemas de equações diferenciais parciais cujas soluções podem ser interpretadas, em algum sentido, como "quantidades minimizantes", ou que caracterizam, por exemplo, os diversos níveis de energia em um átomo (no caso de múltiplas soluções).

O grande truque em problemas variacionais consiste em escolher adequadamente uma classe de funções para então, definir determinado funcional (veja por exemplo o subconjunto $\mathbf{M}$ escolhido no Capítulo 7).

Uma das técnicas padrões para encontrar "quantidades que minimizam" (ou pontos críticos em geral), se dá ao considerar um fluxo gradiente (veja a seção A.4), que deforma um determinado dado inicial na direção de maior decrescimento da energia. Mapas harmônicos podem ser obtidos dessa maneira.

O primeiro grande estudo sobre mapas harmônicos como funções entre variedades riemannianas foi realizado por Eells e Sampson. Em [ES64] eles mostraram que o fluxo gradiente associado a uma generalização do Funcional de Dirichlet, considerado na equação do calor, culmina em soluções cujos pontos críticos são mapas harmônicos.

O objetivo de introduzir tais mapas nesta tese se dá com o intuito de motivar tanto os Fluxos 
de Curvatura Média quanto os Fluxos de Ricci, para os quais, ou a com relação a esses, obtivemos alguns resultados: Lema 2, Teorema 3.3.1 e Teorema 3.3.6.

\subsubsection{Mapas harmônicos e fluxos de curvatura média}

Sejam $(M, g)$ e $(N, h)$ variedades riemannianas fechadas. Um mapa harmônico em $M$ consiste numa aplicação suave $f:(M, g) \rightarrow(M, h)$ que é ponto crítico do Funcional de Dirichlet

$$
E(f):=\int_{M}|d f|^{2} d \mu_{g}
$$

onde $d \mu_{g}:=\sqrt{g} d x^{1} \wedge \ldots \wedge d x^{n}, \operatorname{com} g:=\operatorname{det}\left(g_{i j}\right)$ e $|\cdot|$ é a norma induzida no fibrado $T^{*} M \otimes f^{*}(T N)$.

Dada uma função suave $f:(M, g) \rightarrow(N, h)$, definimos o laplaciano de $f$ por

$$
\Delta_{g, h} f:=\operatorname{tr}_{g} \nabla d f
$$

onde $\nabla$ é a conexão induzida em $T M \otimes f^{*}(T N)$.

Pode-se mostrar que $f$ é um mapa harmônico se, e somente se, $\operatorname{tr}_{g} \nabla d f=0$.

Definição 31. Uma aplicação suave $f:(M, g) \rightarrow(N, h)$ é dita um mapa harmônico se $\Delta_{g, h} f=0$.

Assuma que $f:(M, g) \rightarrow(N, h)$ seja uma imersão isométrica. Então verifica-se que $\Delta_{g, h} f$ é exatamente o vetor de curvatura média $H$ (equação (3.7)) associado a essa imersão. Portanto, fixe uma métrica riemanniana $h \mathrm{em} N$. Denote por $\mathcal{I}(M ; N) \subset C^{\infty}(M ; N)$ o conjunto de todas as imersões isométricas de $M$ em $N$. Pelo Teorema do Mergulho de Nash podemos mergulhar isometricamente $(N, h)$ em $\mathbb{R}^{L}$ para algum $L$. Assim, é possível definir uma estrutura de espaço de Hilbert em $\mathcal{I}(M, N)$ via fórmula

$$
\left(f, f^{\prime}\right):=\int_{M}\left\langle f, f^{\prime}\right\rangle d \mu_{g}
$$

onde $\langle\cdot, \cdot\rangle$ denota o produto interno euclidiano em $\mathbb{R}^{L}$.

Ao calcular variações de $\left.E\right|_{\mathcal{I}(M ; N)}$ conclui-se que o gradiente de $E$ em $f$, denotado por grad $E(f)$, é precisamente $-\Delta_{g, h} f$. Assim, definimos o fluxo de curvatura média (MCF, de maneira curta) de $f_{0} \in \mathcal{I}(M ; N)$ como sendo solução do problema de valor inicial

$$
\left\{\begin{array}{l}
\partial_{t} f(t, p)=\Delta_{g, h} f(t, p)=H(t, p), \\
f(0, p)=f_{0}(p), \forall p \in M .
\end{array}\right.
$$

Note portanto que o fluxo de curvatura média é um exemplo de fluxo gradiente. Ainda mais, podemos notar que $f \in \mathcal{I}(M ; N)$ é uma imersão isométrica mínima se, e somente se, for ponto crítco do Funcional de Dirichlet (3.26).

No artigo [ACG20], escrito em colaboração com os Professores Marcos Alexandrino e Ícaro Gonçalves, obtivemos alguns resultados sobre a convergência de fluxos de curvatura média associados à folheações singulares riemannianas em ambientes não-compactos, sendo uma importante generalização de [AR16].

Não é a intenção do candidato, entranto, expor aqui todo o conteúdo do artigo, uma vez que o autor líder (e que contribuiu majoritariamente) é o Prof. Alexandrino. Ademais, uma exposição completa demandaria uma incursão grande sobre diversos outros assuntos, como métricas de Sasaki, folheações linearizadas, etc.

No entanto, é possível, fazendo uso das ferramentas apresentadas neste capítulo, apresentar uma demonstração simples para alguns dos resultados obtidos, nos quais o candidato trabalhou de forma decisiva.

Seja $(M, g)$ uma variedade riemanniana completa munida de uma folheação singular riemanniana $\mathcal{F}$ cujas folhas são fechadas. Seja $L_{0} \in \mathcal{F}$ uma folha regular. Por definição, $L_{0}$ é uma variedade injetivamente imersa em $M$. Logo, seja $\iota:\left(L_{0}, g_{L_{0}}\right) \rightarrow(M, g)$ a imersão isométrica de $L_{0}$ em $M$ 
induzida pela inclusão. Seja $f(t, p), p \in L_{0}$, o fluxo de curvatura média (equação (3.27)) com condição inicial $\iota$. No que segue, abusaremos notação e nomenclatura ao considerar a imagem do mapa $(t, p) \mapsto f(t, p)$, denotada por $L(t)$, como o fluxo de curvatura média de $L_{0}$.

Teorema 3.3.1. Sejam $(M, g)$ uma variedade riemanniana completa munida de uma folheação singular riemanniana com folhas fechadas $\mathcal{F}$. Assuma que

1. M tenha curvatura seccional limitada;

2. O operador formato ao longo de cada folha $L \in \mathcal{F}$ seja limitado;

3. O quociente $M / \mathcal{F}$ seja compacto.

Seja $N$ uma sumariedade imersa contida em uma folha regular. Assuma que a dimensão de $N$ seja maior que a dimensão das folhas singulares, e que o fluxo de curvatura média $N(t)$ seja a restrição de um fluxo básico (com respeito a $\mathcal{F}$ ) no estrato regular, cujo intervalo máximo de existência é $[0, T), T<\infty$. Então $N(t)$ converge a uma folha singular no espaço das folhas.

Considere uma folha $L \in \mathcal{F}$. Denote por $r(p):=d(L, p)$ a função distância até $L$. Lembrando que as folhas de $\mathcal{F}$ são localmente equidistantes, se $L(t)$ está contida numa vizinhança tubular de modo que $r$ restrita a essa seja suave, então a função $r(t)=d(L, L(t))$ fica bem definida, isto é, independente de escolha de um ponto específico em $L(t)$. Portanto, fixe $p \in L_{0}$. Então,

$$
\begin{aligned}
\frac{d}{d t} r(t) & =\frac{d}{d t} r \circ f(t, p) \\
& =g\left(\nabla r\left(f_{t}\right), \partial_{t} f(t, p)\right) \\
& =g\left(\nabla r\left(f_{t}\right), H(t, p)\right) \\
& =\operatorname{tr} S_{\nabla r(t)},
\end{aligned}
$$

onde $S_{\nabla r(t)}$ denota o operador de formato referente a folha $L(t)$ calculado em $\nabla r(t)$ (equação (3.7)).

A ideia principal para a prova do Teorema 3.3.1 consiste em encontrar bacias de atração para o fluxo $N(t)$. Precisamente:

Lema 1 (Bacias de atração). Sejam $(M, \mathcal{F}, N, g)$ como nas hipóteses do Teorema 3.3.1. Seja $L_{q} \in \mathcal{F}$ uma folha singular. Então existe $\epsilon>0$ tal que,

1. Existe uma constante $C_{1} \in \mathbb{R}$, que depende somente de $\operatorname{Tub}_{\epsilon}\left(L_{q}\right)$, tal que se para algum $t_{0}>0$ valer que $N\left(t_{0}\right) \subset \operatorname{Tub}_{\epsilon}\left(L_{q}\right)$, então

$$
r^{2}\left(t_{0}\right)-r^{2}(t) \geq C_{1}^{2}\left(t-t_{0}\right), \forall t \in\left[t_{0}, T\right) .
$$

Em particular, $T<\infty$.

2. Para todo $t \in\left[t_{0}, T\right), N(t) \subset \operatorname{Tub}_{\epsilon}\left(L_{q}\right)$.

Para a demonstração do Lema 1 nos apoiaremos no seguinte resultado, que acreditamos ser útil no contexto de teoria de imersões.

Lema 2. Seja $(M, g)$ uma variedade riemanniana com curvatura seccional limitada, ou seja, existe uma constante $\Lambda \in \mathbb{R}$ tal que $\left|K_{g}\right| \leq \Lambda$. Seja $L$ uma subvariedade mergulhada em $M$ com operador formato limitado. Assuma que esteja bem definida uma vizinhança tubular $\operatorname{Tub}_{\epsilon_{0}}(L)$ para algum $\epsilon_{0}>0$. Então, dado um inteiro positivo $k$, existem constantes positivas $C, D$ e $c_{1}, c_{2}$, tais que para cada $\epsilon \in\left(0, \epsilon_{0}\right)$ e para cada subvariedade imersa $N \subset \partial \operatorname{Tub}_{\epsilon}(L)$ satisfazendo $\operatorname{dim} N=\operatorname{dim} L+k$, as seguintes desigualdades são verdadeiras

$$
-\frac{D}{r(p)}-c_{2} \leq \operatorname{tr}\left(S_{\nabla r}\right) \leq-\frac{C}{r(p)}+c_{1}
$$

onde $S_{\nabla r}$ é o operador formato da subvariedade imersa $N \subset \partial \operatorname{Tub}_{\epsilon}(L)$ e $r(p)=d(L, p)$ é a função distância até $L$. 
Observação. Não conseguimos encontrar o Lema 2 na literatura, mas esse pode ser visto como uma generalização natural do Teorema 27 em [Pet06a, Chapter 6].

A demonstração do Lema 2 é baseada em métodos de comparação tradicionais em geometria riemanniana, combinados com construções via produtos verticais. Comecemos com uma breve digressão.

Considere duas triplas $\left(M_{1}^{m}, L_{1}^{n}, \gamma_{1}\right)$ e $\left(M_{2}^{m}, L_{2}^{n}, \gamma_{2}\right)$, onde $\left(M_{i}, g_{i}\right)$, são variedades riemannianas, $L_{i}$, são subvariedades mergulhadas em $M_{i}$ e $\gamma_{i}$, é uma geodésica unitário ortogonal a $L_{i}$ em $\gamma_{i}(0)$. Assuma também que $U_{i}$, seja uma vizinhança tubular de $L_{i}$ com raio $\epsilon_{0}$ de modo que as funções distância $r_{i}: U_{i} \backslash L_{i} \rightarrow \mathbb{R}$, definidas por $r_{i}(p)=d_{i}\left(L_{i}, p\right)$, sejam funções suaves. Precisamente, estamos assumindo que $\left.\gamma_{i}\right|_{\left[0, \epsilon_{0}\right]}$ não contenha pontos focais a $L_{i}$.

Denote por $\mathcal{V}^{i} \subset \Gamma\left(T M_{i}\right)$ o subspaço dos campos de vetores que são ortogonais a $\gamma_{i}$ e que começam tangentes a $L_{i}$.

Lema 3. Assuma que

(a) $\sup _{e_{1}(t) \in \nu_{1}(s)} K_{1}\left(e_{1}, \gamma_{1}^{\prime}\right) \leq \inf _{e_{2}(s) \in \nu_{2}(t)} K_{2}\left(e_{2}, \gamma_{2}^{\prime}\right)$ (onde $\left.\left|e_{i}\right|=1\right)$.

(b) $\max _{j} \lambda_{1}^{j} \leq \min _{j} \lambda_{2}^{j}$ onde $\lambda_{i}^{j}$ é uma curvatura principal associado ao operador formato $S_{\gamma_{i}^{\prime}(0)}$.

Então para todo $\epsilon \in\left[0, \epsilon_{0}\right)$ existe um isomorfismo $\theta(\epsilon): \nu_{1}(\epsilon) \rightarrow \nu_{2}(\epsilon)$ tal que

$$
\text { Hess } r_{2}\left(\theta(\epsilon) X_{1}, \theta(r) X_{1}\right) \leq \operatorname{Hess} r_{1}\left(X_{1}, X_{1}\right)
$$

O Lema 3 será usado na demonstração do Lema 2. A ideia consiste em construir variedades modelo $\widetilde{M}$, via produtos verticais, de modo que se $M, N, L$ são como nas hipóteses do Lema 2, então $\widetilde{M}$ faz, ora o papel de $M_{1}$, ora o papel de $M_{2}$, no Lema 3. A construção é bastante simples, de modo que $\widetilde{M}$ é um produto vertical cuja base consiste ou em uma esfera $S_{R}^{d}$, ou em um espaço hiperbólico $H_{R}^{d}$, onde $d$ é a codimensão de $L$ em $M$, munidos de suas métricas padrões. Em ambos os casos, a fibra em $\widetilde{M}$ será tomada como folha $L$.

Nesse tipo de comparação, é necessário entender o comportamento dos vetores $\theta\left(X_{1}\right)$ nos casos em que $X_{1}$ é tangente/perpendicular a um slice $S_{1}$ em $M_{1}$. O próximo lema garante que sob a hipótese de curvatura limitada, o transporte paralelo ao longo de $\gamma_{1}$ de um vetor que começa tangente a $L_{1}$ é tal que sua imagem por $\theta$ possui projeção bem controlado no slice $S_{2}$, ao menos para tempo curto.

Lema 4. Assuma que $\sup _{e_{1}(s) \in \nu_{1}(s)} K_{1}\left(e_{1}, \gamma_{1}^{\prime}\right) \leq \inf _{e_{2}(s) \in \nu_{2}(s)} K_{2}\left(e_{2}, \gamma_{2}^{\prime}\right)$ onde $\nu_{i}(s)$ denota o espaço dos vetores normais a $\gamma_{i}$ em $s$, e que $\left|e_{i}\right|=1$. Sejam $V_{i}$ campos de vetores paralelos e unitários ao longo $\gamma_{i}$ tais que $V_{i}(0) \in T_{\gamma_{i}(0)} L_{i}$. Sejam $J_{i}$ campos de Jacobi ao longo de $\gamma_{i}$ tais que $J_{i}(0)=0$, $J_{i}^{\prime}(0) \in T_{\gamma_{i}(0)} S_{i}$, onde $S_{i}:=\exp _{\gamma_{i}(0)}\left(\nu_{\gamma_{i}(0)} L_{i} \cap B_{\epsilon}(0)\right)$ é um slice em $\gamma_{i}(0)$ e $\left|J_{1}^{\prime}(0)\right|=\left|J_{2}^{\prime}(0)\right|$. Então, existe uma constante $C$, dependendo somente de $\sup _{[0, \epsilon]}\left|R_{2}\right|$ e $\sup _{[0, \epsilon]}\left|J_{1} / s\right|$, de modo que para cada $s \in(0, \epsilon)$

$$
\left|g_{2}\left(V_{2}(s), J_{2} / s\right)\right| \leq C s^{2},
$$

onde $R_{2}$ denota o tensor de Riemann associado à métrica $g_{2}$.

Demonstração. Veja [ACG19, Lemma 6.6, p. 14, v1].

Demonstração do Lema 2. Começamos com uma vizinhança tubular $\operatorname{Tub}_{\epsilon_{0}}(L)$ ao redor de $L$ de modo que o mapa $p \mapsto d(p, L)$ seja suave em $\operatorname{Tub}_{\epsilon_{0}}(L) \backslash L$. Assuma que $\epsilon_{0}$ seja tal que nenhuma geodésica que comece ortogonal a $L$ tenha pontos focais em $\operatorname{Tub}_{\epsilon_{0}}(L)$. Denote por $s \rightarrow \gamma(s)$ o segmento geodésico parametrizado por comprimento de arco que é ortogonal a $L$ em $s=0$ e que realiza a distância entre $L$ e $p \in N$ i.e, $r(p)=d(p, L)=s$.

Seja $\mathcal{B}:=\left\{e_{n}\right\}$ um referencial ortonormal tangente a $N$ em $\gamma(s)=p$. Então,

$$
-\operatorname{tr}\left(S_{\nabla r}\right)=\sum_{n=1}^{\operatorname{dim} N} g\left(\nabla_{e_{n}} \nabla r, e_{n}\right)=\sum_{n=1}^{\operatorname{dim} N} \operatorname{Hess} r\left(e_{n}, e_{n}\right) .
$$


Seja $S_{R}^{d}$ a esfera $d$-dimensional de raio $R$ munida da métrica redonda, onde $d$ é a codimensão de $L$ em $M$. Considere o produto vertical $S_{R}^{d} \times_{e^{2 \phi}} L$ definido pela métrica

$$
\widetilde{g}=g_{S_{R}^{d}} \times e^{2 \phi} g_{L},
$$

onde $g_{L}$ é a métrica induzida em $L$ por $g$, e

$$
\phi(p):=\lambda d^{2}\left(p, q_{0}\right), \lambda \in \mathbb{R}, q_{0} \in S_{R}^{d} .
$$

Afirmação 1. Existem números reais $R>0>\lambda$ e um ponto $q_{0} \in S_{R}^{d}$ tais que as variedades riemannianas

$$
\begin{gathered}
\left(M_{1}, g_{1}\right):=\left(\operatorname{Tub}_{\epsilon}(L), g\right), L_{1}:=L \\
\left(M_{2}, g_{2}\right):=\left(S_{R}^{d} \times L, \tilde{g}\right), L_{2}:=\left\{q_{N}\right\} \times L
\end{gathered}
$$

são como nas hipóteses do Lema 3, onde $q_{N} \in S_{R}^{d}$ denota o Polo Norte.

Assuma por instante a veracidade da Afirmação 1. Seja $s \rightarrow \gamma_{2}(s)$ em $M_{2}$ o segmento geodésico parametrizado por comprimento de arco que começa perpendicular a $L_{2}$ em $q_{N}$ (i.e, $\left.\gamma_{2}(0)=q_{N}\right)$. Seja $U_{2}:=\operatorname{Tub}_{\epsilon_{0}}\left(\left\{q_{N}\right\} \times L\right)$ uma vizinhança tubular de $\left\{q_{N}\right\} \times L$ em $M_{2}$ e considere a função distância,

$$
\begin{gathered}
r_{2}: U_{2} \backslash\left(\left\{q_{N}\right\} \times L\right) \rightarrow \mathbb{R}, \\
r_{2}: p \mapsto d_{\widetilde{g}}\left(p,\left\{q_{N}\right\} \times L\right),
\end{gathered}
$$

Para cada $n \in\{1, \ldots, \operatorname{dim} N\}$,

$$
\theta\left(e_{n}\right)=e_{n}^{\top}+e_{n}^{\perp}, e_{n} \in \mathcal{B},
$$

onde $e_{n}^{\top}$ denota a componente de $e_{n}$ que é tangente a $L$ e $e_{n}^{\perp}$ a componente tangente à base do produto vertical.

Pelo Lema de Comparação (Lema 3),

$$
\text { Hess } r\left(e_{n}, e_{n}\right) \geq \operatorname{Hess} r_{2}\left(e_{n}^{\top}, e_{n}^{\top}\right)+\operatorname{Hess} r_{2}\left(e_{n}^{\perp}, e_{n}^{\perp}\right) \text {. }
$$

Como a base do produto vertical é a esfera redonda,

$$
\text { Hess } r_{2}\left(e_{n}^{\perp}, e_{n}^{\perp}\right)=\frac{1}{R} \cot \left(\frac{r_{2}}{R}\right)\left|e_{n}^{\perp}\right|^{2} .
$$

Ademais, pela equação (3.20)

$$
\text { Hess } r_{2}\left(e_{n}^{\top}, e_{n}^{\top}\right)=2 \lambda e^{2 \lambda\left(s+s_{0}\right)^{2}}\left(s+s_{0}\right)\left|e_{n}^{\top}\right|^{2}, \lambda<0 .
$$

$$
\text { Hess } r\left(e_{n}, e_{n}\right) \geq 2 \lambda\left(\epsilon_{0}+s_{0}\right) e^{2 \lambda\left(\epsilon_{0}+s_{0}\right)^{2}}\left|e_{n}^{\top}\right|^{2}+\frac{1}{R} \cot \left(\frac{r_{2}}{R}\right)\left|e_{n}^{\perp}\right|^{2} .
$$

Somando os termos e utilizando a definição de $\operatorname{tr}\left(S_{\nabla r}\right)$ obtemos

$$
\operatorname{tr}\left(S_{\nabla r}\right) \leq-2 \lambda\left(\epsilon_{0}+s_{0}\right) e^{2 \lambda\left(\epsilon_{0}+s_{0}\right)^{2}} \sum_{n}\left|e_{n}^{\top}\right|^{2}-\frac{1}{R} \cot \left(\frac{r_{2}}{R}\right) \sum_{n}\left|e_{n}^{\perp}\right|^{2} .
$$

Assim,

$$
\operatorname{tr}\left(S_{\nabla r}\right) \leq-2 \lambda\left(\epsilon_{0}+s_{0}\right) e^{2 \lambda\left(\epsilon_{0}+s_{0}\right)^{2}} \sum_{n}\left|e_{n}^{\top}\right|^{2}-\frac{1}{R} \cot \left(\frac{r}{R}\right) \sum_{n}\left|e_{n}^{\perp}\right|^{2}
$$


Expandindo o polinômio de Taylor, $\cot \left(\frac{r}{R}\right)=\frac{R}{r}-o(r)$, onde $o(r)>0$. Logo,

$$
\operatorname{tr}\left(A_{\nabla r}\right) \leq-2 \lambda\left(\epsilon_{0}+s_{0}\right) e^{2 \lambda\left(\epsilon_{0}+s_{0}\right)^{2}} \sum_{n}\left|e_{n}^{\top}\right|^{2}-\frac{1}{R}\left(\frac{R}{r}-o\left(\epsilon_{0}\right)\right) \sum_{n}\left|e_{n}^{\perp}\right|^{2} .
$$

Como para todos $n \in\{1, \ldots, \operatorname{dim} N\}$, vale que $\left|e_{n}^{\top}\right|,\left|e_{n}^{\perp}\right| \leq 1$, definimos

$$
c_{1}:=-2 \lambda\left(\epsilon_{0}+s_{0}\right) e^{2 \lambda\left(\epsilon_{0}+s_{0}\right)^{2}} \operatorname{dim} N+\frac{1}{R} o\left(\epsilon_{0}\right) \operatorname{dim} N .
$$

Note que, como consequência da hipótese, $c_{1}$ não depende da dimensão de $N$ pois $\operatorname{dim} N=\operatorname{dim} L+$ $k, \exists k \in \mathbb{N}$.

Portanto

$$
\operatorname{tr}\left(S_{\nabla r}\right) \leq c_{1}-\frac{1}{r} \sum_{n}\left|e_{n}^{\perp}\right|^{2}
$$

Reduzindo $\epsilon_{0}$ se necessário e utilizando o Lema 4, concluímos que existe $C>0$ tal que

$$
\sum_{n}\left|e_{n}^{\perp}\right|^{2} \geq C
$$

de onde,

$$
\operatorname{tr}\left(S_{\nabla r}\right) \leq c_{1}-\frac{C}{r(p)}
$$

o que conclui a demonstração de um lado das desigualdades. De forma análoga, substituindo $\left(M_{1}, g_{1}\right)=\left(H_{R}^{d}, \widetilde{g}\right),\left(M_{2}, g_{2}\right)=(M, g)$, pode-se provar uma afirmação análoga à Afirmação 1 , que demonstraremos agora.

Demonstração da Afirmação 1. Seja $\left\{\widetilde{e}_{l}\right\}$ um referencial ortonormal paralelo ao longo da geodésica unitária $\widetilde{\gamma}$ em $M_{2}$, que é perpendicular a $L_{2}$ em $\widetilde{\gamma}(0)$.

Como $\widetilde{\gamma}$ é parametrizada por comprimento de arco, definindo $q_{0}=\tilde{\gamma}\left(-s_{0}\right)$ temos que existe $s_{0}>0$ (a ser determinado) tal que

$$
\phi(\widetilde{\gamma}(s))=\lambda d^{2}\left(\widetilde{\gamma}(s), q_{0}\right)=\lambda d^{2}\left(\widetilde{\gamma}(s), \widetilde{\gamma}\left(-s_{0}\right)\right)=\lambda\left(s+s_{0}\right)^{2},
$$

Logo

$$
\left\langle\nabla \phi, \widetilde{\gamma}^{\prime}(s)\right\rangle=\frac{d}{d s} \phi(\widetilde{\gamma}(s))=\lambda \frac{d}{d s}\left(s+s_{0}\right)^{2}=2 \lambda\left(s+s_{0}\right)
$$

Ainda mais

$$
\text { Hess } \phi(s)=\lambda \frac{d^{2}}{d s^{2}}\left(s+s_{0}\right)^{2}=2 \lambda .
$$

Usando as equação (3.2.3) concluímos que

$$
\begin{aligned}
\widetilde{K}\left(\widetilde{e}_{l}, \widetilde{\gamma}^{\prime}(s)\right) & =-e^{2 \lambda\left(s+s_{0}\right)^{2}}\left(\left\langle\nabla \phi, \widetilde{\gamma}^{\prime}(s)\right\rangle^{2}+\operatorname{Hess} \phi\left(\widetilde{\gamma}^{\prime}(s)\right)\right) \\
& =-e^{2 \lambda\left(s+s_{0}\right)^{2}}\left(4 \lambda^{2}\left(s+s_{0}\right)^{2}+2 \lambda\right) .
\end{aligned}
$$

Ademais

$$
\widetilde{g}\left(\widetilde{\sigma}\left(\widetilde{e}_{l}, \widetilde{e}_{l}\right), \widetilde{\gamma}^{\prime}(s)\right)=-2 e^{2 \lambda\left(s+s_{0}\right)^{2}} \lambda\left(s+s_{0}\right) .
$$

Escolha $R$ tal que $\frac{1}{R^{2}}>\Lambda$. Segundo a equação (3.34), necessariamente devemos tomar $\lambda<0$. Denote por $\lambda_{L}>0$ o limitante das curvaturas principais em $L$. Os parâmetros $s_{0}, \lambda$ devem ser tomados levando em conta as seguintes desigualdades,

$$
-4 \lambda^{2}\left(s+s_{0}\right)^{2}-2 \lambda>\Lambda, \forall s \in\left[0, \epsilon_{0}\right] .
$$




$$
\begin{aligned}
4 \lambda^{2}\left(s+s_{0}\right)^{2}+2 \lambda & <-\Lambda \\
4 \lambda^{2}\left(s+s_{0}\right)^{2} & <-\Lambda-2 \lambda .
\end{aligned}
$$

Como essas devem valer para todo $s \geq 0$, é suficiente provar que

$$
4 \lambda^{2}\left(\epsilon_{0}+s_{0}\right)^{2}<-\Lambda-2 \lambda .
$$

Ademais, gostaríamos que,

$$
-2 e^{2 \lambda\left(s+s_{0}\right)^{2}} \lambda\left(s+s_{0}\right)>\lambda_{L} .
$$

Como $\lambda<0$, é suficiente que valha

$$
-2 \lambda s_{0} e^{2 \lambda\left(\epsilon_{0}+s_{0}\right)^{2}}>\lambda_{L}
$$

Logo, os parâmetros procurados devem satisfazer

$$
e^{-2 \lambda\left(\epsilon_{0}+s_{0}\right)^{2}} \lambda_{L}<-2 \lambda s_{0}<\sqrt{-\Lambda-2 \lambda}+2 \lambda \epsilon_{0},
$$

i.e,

$$
-e^{-2 \lambda\left(\epsilon_{0}+s_{0}\right)^{2}} \frac{\lambda_{L}}{2 \lambda}<s_{0}<-\frac{\sqrt{-\Lambda-2 \lambda}}{2 \lambda}-\epsilon_{0} .
$$

Não é difícil se convencer de que existe uma escolha tomando $|\lambda|$ grande o suficiente e $s_{0}$ pequeno o suficiente de modo que a desigualdade (3.37) seja cumprida, concluindo o afirmado.

Demonstração do Lema 1. Seja $L_{q} \in \mathcal{F}$ uma folha singular. Dado que $N(t)$ tem dimensão constante para todo $t<T$, podemos aplicar o Lema 2 considerando $L=L_{q}, N=N(t)$. Assim, existem uma vizinhança tubular $\operatorname{Tub}_{\epsilon_{0}}\left(L_{q}\right), \epsilon_{0}>0$, e constantes $C, c_{1}>0$, só dependendo de $\operatorname{Tub}_{\epsilon_{0}}\left(L_{q}\right)$, tais que

$$
\operatorname{tr} S_{\nabla r(t)} \leq-\frac{C}{r(t)}+c_{1}
$$

Assim, escolha $\epsilon<\min \left\{\epsilon_{0}, \frac{C}{c_{1}}\right\}$ e defina $\frac{C_{1}^{2}}{2}:=C-\epsilon c_{1}$. De acordo com a equação (3.31),

$$
r^{\prime}(t) \leq-\frac{C_{1}^{2}}{2 r(t)}
$$

Logo, integrando em ambos os lados,

$$
r^{2}(t)-r^{2}\left(t_{0}\right) \leq-C_{1}^{2}\left(t-t_{0}\right)
$$

Portanto

$$
r^{2}\left(t_{0}\right)-r^{2}(t) \geq C_{1}^{2}\left(t-t_{0}\right)
$$

o que conclui a demonstração.

Demonstração do Teorema 3.3.1. Para terminar a demonstração do Teorema 3.3.1, seja $\pi: M \rightarrow$ $M / \mathcal{F}$ a projeção canônica. Como para cada $t>0, \pi(N(t))$ está contida em um compacto e $T<\infty$, o limite $N(t)$ deve estar contido no estrato singular (veja [PT88, Proposition 9.1.4, p. 183]). Seja portanto $L_{q}$ uma folha singular no conjunto limite. Tome $t_{n} \subset[0, T)$ tal que $t_{n} \rightarrow T$ e $\pi\left(N\left(t_{n}\right)\right) \rightarrow$ $\pi\left(L_{q}\right)$. Então, pela definição de limite, dado $\epsilon_{0}>0$ suficientemente pequeno, existe $t_{n_{0}}$ tal que $N(t) \subset \partial \operatorname{Tub}_{\epsilon_{0}}(L)$. Assim, segundo a Afirmação 1, existe $\epsilon>0$ para todo $t>t_{n_{0}}, L(t) \subset \operatorname{Tub}_{\epsilon}\left(L_{q}\right)$. Como $\epsilon$ é arbitrário, o resultado segue. 


\subsubsection{Fluxos de Ricci e Solitons de Ricci}

Nesta breve subseção introduziremos de forma básica o conceito de fluxos de Ricci, com o intuito de bem motivar e apresentar alguns dos Solitons de Ricci, que serão realizados como base em novas famílias de fibrados com curvatura de Ricci positiva, cujas fibras podem ser escolhidas como diversas variedades exóticas (veja o Capítulo 6).

Na década de 80, o matemático Richard Hamilton iniciou um programa para solução da conjectura de Poincaré:

Conjectura 3.3.2 (Poincaré). Toda variedade fechada $M^{3}$ e simplesmente conexa de dimensão 3 é homeomorfa à esfera $S^{3}$.

Com influência dos trabalhos de Eells e Sampson sobre mapas harmônicos, uma possível abordagem para o problema consiste em mostrar que toda 3-variedade admite uma métrica de curvatura seccional constante e positiva. Hamilton obteve sucesso ao provar esse resultado assumindo que $M^{3}$ admitia uma métrica $g$ curvatura de Ricci estritamente positiva (veja [Ham82]). A ideia inicial era evoluir a métrica $g$ como um "fluxo de calor" com o intuito de uniformizar a geometria.

A equação inicial cogitada por Hamilton é a seguinte:

$$
\frac{\partial g}{\partial t}(t)=-2 \operatorname{Ric}(g)+\operatorname{scal}(t) \frac{2}{n} g,
$$

derivada como fluxo gradiente associado ao funcional

$$
\mathcal{F}(g):=\int_{M} \operatorname{scal}_{g} d \mu_{g}
$$

(Veja, e.g,[Top06, Chapter 6, p. 67-68]).

No entanto, a equação (3.38) implica numa evolução ruim para a curvatura escalar. De fato, essa é uma equação do calor do tipo backward, e portanto, não admite existência de solução em $\mathbb{R}_{>0}$.

Como o problema se dá pela evolução da curvatura escalar, pode-se considerar a média dessa em $M$,

$$
r:=\int_{M} \operatorname{scal}(g) d \mu_{g} / \int_{M} d \mu_{g}
$$

obtendo uma nova equação,

$$
\frac{\partial g}{\partial t}(t)=-2 \operatorname{Ric}(g)+\frac{2 r}{n} g
$$

A equação (3.39) é conhecida como fluxo de Ricci normalizado. Na literatura, a equação disseminada como fluxo de Ricci é dada por,

$$
\frac{\partial g}{\partial t}=-2 \operatorname{Ric}(g)
$$

A equação (3.39) tem nomenclatura justificava uma vez que evita problemas como colapso de variedades em tempo finito. De fato, se considerarmos a esfera padrão $S^{n}$ com a métrica redonda $g_{0}$, e procurarmos uma solução para a equação (3.40) na forma $g(t)=r^{2}(t) g_{0}$, como $\operatorname{Ric}(g(t))=$ $\operatorname{Ric}\left(g_{0}\right)=(n-1) g_{0}$, obtemos

$$
\left(2 r r^{\prime}\right)(t) g_{0}=-2 \operatorname{Ric}\left(g_{0}\right)=-2(n-1) g_{0},
$$

Assim ,

$$
r r^{\prime}(t)=-(n-1)
$$

ou seja,

$$
\begin{aligned}
& r^{2}(t)=-2(n-1) t+1 \\
& r(t)=\sqrt{-2(n-1) t+1}
\end{aligned}
$$


de onde

$$
g(t)=\sqrt{-2(n-1) t+1} g_{0}
$$

e a variedade colapsa até o instante $t=\frac{1}{2(n-1)}$.

Hamilton esperava encontrar uma formulação de fluxo gradiente para a equação (5.57), que só foi descoberta por Perelman em [Per02].

Teorema 3.3.3 (Perelman). Seja go uma métrica arbitrária em uma variedade fechada M. Então,

1. O funcional

$$
\mathcal{F}^{m}(g)=\int_{M}\left(\operatorname{scal}(g)+|\nabla f|^{2}\right) \omega
$$

tem como gradiente

$$
\operatorname{grad} \mathcal{F}^{m}(g)=-\left(\operatorname{Ric}(g)+\operatorname{Hess}_{g}(f)\right)
$$

2. Existem $T>0$ e uma $n$-forma $\omega$ tais que o fluxo gradiente associado ao funcional $\mathcal{F}^{m}$, onde $f$ é determinada por $\omega=e^{-f} d \mu(g)$, tem solução em $[0, T)$. Ainda mais, esse fluxo é mapeado difeomorficamente no fluxo de Ricci via um difeomorfismo que depende de $t$.

Demonstração. Veja [Top06, Chapter 6].

Por fim, uma justificativa para o fluxo de Ricci ser entendido como um análogo à equação de evolução para o calor se dá pelo fato que, em coordenadas harmônicas, a equação (5.57) tem a forma

$$
\partial_{t} g_{i j}(t)=3 \Delta g_{i j}(t)+o\left(\Gamma_{i j}\right),
$$

onde o termo $o\left(\Gamma_{i j}\right)$ envolve também derivadas de segunda ordem de $g_{i j}$, mas no ponto onde estão centradas tais coordenadas, pode-se assumir que $o\left(\Gamma_{i j}\right)=0$.

Uma métrica riemanniana completa $g$ em uma variedade suave $M$ é chamada Soliton de Ricci se existir um campos de vetores suave $X \in \Gamma(T M)$ e uma constante $\rho \in \mathbb{R}$ de modo que valha a seguinte equação

$$
\operatorname{Ric}(g)+\frac{1}{2} \mathcal{L}_{X} g=\rho g,
$$

onde $\mathcal{L}$ denota a derivada de Lie.

Se $X=\nabla f$, onde $f$ é uma função suave, a equação (3.43) se torna

$$
\operatorname{Ric}(g)+\operatorname{Hess} f=\rho g,
$$

e o soliton de Ricci é dito do tipo gradiente.

É possível derivar outras equações a partir de (3.44). Por exemplo, tomando o traço em ambos os lados,

$$
\operatorname{scal}(g)+\Delta f=n \rho,
$$

onde $\operatorname{scal}(g)$ denota a curvatura escalar de $g$ e $n$ denota a dimensão de $M$.

Mais ainda, pode ser provado que se a $\operatorname{EDP}(3.44)$ é satisfeita, então existe $\widetilde{C} \in \mathbb{R}$ tal que

$$
\operatorname{scal}(g)+|\nabla f|^{2}=2 \rho f+\widetilde{C} .
$$

(Veja por exemplo [Cao09, Lemma 1.1, pg 2])

Solitons de Ricci (RS, de maneira curta) são nomeados na literatura de acordo com o sinal de $\rho$. Um RS é dito steady se $\rho=0$, expanding se $\rho<0$ e shrinking se $\rho>0$.

Esses objetos foram introduzidos em [Ham88] como soluções particulares da equação (5.57), onde a métrica $g$ original evolui (no tempo) via difeomorfismos de $g$. Sua importância foi posteriormente demonstrada, por exemplo, por Perelman, cuja classificação de Solitons de Ricci do tipo shrinking em dimensão 3 levou à solução da conjectura de Poincaré, e em [Ses04], onde é provado que solitons de Ricci do tipo gradiente são os limites de blow-up do fluxo de Ricci. 
Note ainda que Solitons de Ricci do tipo steady são pontos críticos do funcional definido por Perelman (equação 3.42). Ademais, a equação (3.44) determina $f$ a menos de constante, i.e, se $f$ satisfaz a EDP (3.44), então $f+C, C \in \mathbb{R}$, também satisfaz. Assim, Solitons de Ricci de tipo gradiente serão denotados por $(M, g, f)$ com o intuito de enfatizar a função representante escolhida.

Em [MW17] prova-se que Solitons de Ricci do tipo gradiente com curvatura seccional positiva são sempre compactos. Mais ainda, um Soliton de Ricci do tipo shrinking com curvatura seccional não-negativa e curvatura de Ricci positiva também é compacto.

Em variedades compactas, um Soliton de Ricci do tipo gradiente é Einstein se, e somente se, for rígido. I.e, for isométrico a $\mathbb{R}^{k} \times_{\Gamma} N$ onde $N$ é uma variedade de Einstein, $f=\frac{\rho}{2}|x|^{2}$ é função definida no fator euclidiano e $\Gamma$ age livremente em $N$ por transformações ortogonais de $\mathbb{R}^{k}$. Em particular, Solitons de Ricci homogêneos são sempre rígidos (veja a discussão em[FLGR16]).

Definição 32. Um Soliton de Ricci é dito não trivial se não for uma métrica de Einstein.

Doravante, denotaremos por SRS um Soliton de Ricci do tipo Shrinking. Para dimensões $n \geq 4$ sempre existem SRS que não são triviais (veja [Cao09]). Ademais, existe uma classicação de SRS em dimensão maior que 4 com hipótese adicional que esses são localmente conformemente planos (veja [NW07]), ou assumindo que o tensor de Weyl se anule identicamente e que o tensor de Ricci satisfaça uma certa condição integral (veja [PW10]).

Em [Dan11] os autores constroem exemplos de SRS do tipo gradiente que são compactos e tem curvatura de Ricci positiva. Os exemplos por eles obtidos são realizações dos SRS como variedades Fano. Ou seja, variedades de Kähler para as quais a primeira classe de Chern é positiva. Tais métricas sempre tem curvatura de Ricci positiva.

Alguns exemplos construídos no Capítulo 6 são baseados no seguinte teorema.

Teorema 3.3.4 (Dancer-Wang). Sejam $\left(V_{i}, J_{i}, h_{i}\right), 1 \leq i \leq r, r \geq 3$, variedades de Kähler-Einstein que sejam Fano, tenham dimensão complexa $n_{i}$ e primeira classe de Chern $p_{i} \alpha_{i}$, onde $p_{i}>0, e$ $\alpha_{i}$ são classes indivisiveis em $H^{2}\left(V_{i} ; \mathbb{Z}\right)$. Sejam $V_{1}$ e $V_{r}$ espaços projetivos complexos munidos da métrica de Fubini-Study normalizada. Faça $P_{q}$ denotar o $S^{1}$-fibrado principal sobre $V_{1} \times V_{2} \times \ldots V_{r}$ com classe de Euler dada por $-\pi_{1}^{*}\left(a_{1}\right)+\sum_{i=2}^{r-1} q_{i} \pi_{i}^{*}\left(a_{i}\right)+\pi_{r}^{*}\left(a_{r}\right)$.

Suponha ainda que $-\left(n_{1}+1\right) q_{i}<p_{i}$ e que $\left(n_{r}+1\right) q_{i}<p_{i}, \forall 2 \leq i \leq r-1$. Então, existe uma estrutura de SRS compacto do tipo gradiente no espaço total $\bar{M}$ obtido a partir $P_{q} \times_{S^{1}} \mathbb{C P}^{1}$ via blowing $P_{q}$ down para $V_{2} \times \ldots \times V_{r}$ em um fim e $V_{1} \times \ldots \times V_{r-1}$ no outro fim, se, e somente se, para algum $\kappa_{1} \in \mathbb{R}$, a integral

$$
I:=\int_{n_{r}-1}^{n_{r}+1} e^{-2 \kappa_{1}\left(p+n_{1}+1\right)} \prod_{i=1}^{r}\left(p-\frac{p_{i}}{q_{i}}\right)^{n_{i}} d x
$$

se anula. Se $\kappa_{1} \neq 0$, o SRS assim construído é não trivial.

Via o entendimento do comportamento da integral $I$ no Teorema 3.3.4 conclui-se que:

Teorema 3.3.5 (Dancer-Wang). Todas as variedades $\bar{M}$ descritas no Teorema 3.3.4 admitem uma estrutura de SRS compacta de tipo gradiante cuja métrica de SRS é uma métrica de Kähler que também é Fano.

Como variedades de Fano têm curvatura de Ricci positiva, baseando-nos nas construções de produto vertical, no Capítulo 6 construíremos diversos exemplos de variedades com curvatura de Ricci positiva, incluindo fibrados cujas bases são as variedades dadas pelos Teoremas 3.3.4, 3.3.5 e cujas fibras podem ser diversas variedades exóticas. De fato, isso segue como corolário do seguinte Teorema:

Teorema 3.3.6. Seja $(M, g, f)$ um SRS do tipo gradiente compacto e não trivial como no Teorema 3.3.4. Seja F uma variedade satisfazendo as hipóteses do Teorema C. Então existe uma métrica em $F$ tal que a variedade riemanniana $M \times_{e^{2 f}} F$ desenvolve curvatura de Ricci positiva após uma deformação canônica. 
Demonstração. O Teorema $\mathrm{C}$ garante que existe uma métrica $G$-invariante em $F$ com curvatura de Ricci positiva. Por fim, como $g$ tem curvatura de Ricci positiva, o resultado segue como corolário do Teorema 3.2.5.

Para criar certa expectativa no leitor sobre os próximos capítulos, descrevemos brevemente alguns exemplos explícitos descritos em [Dan11].

Exemplo. Existe um estrutura de $S R S(M, g, f)$ compacta, não trivial, e do tipo gradiente; no espaço total de um $\mathbb{C P}^{1}$-fibrado sobre $\mathbb{C P}^{2} \times \mathbb{C P}^{2}$. Ainda mais, $(M, g, f)$ é uma variedade de Kähler que também é Fano.

Exemplo. Em geometria algébrica, uma threefold é uma hipersuperfície de grau 3 em um espaço projetivo. Precisamente, seja $f=f\left(x_{1}, \ldots, x_{n}\right) \in \mathbb{C}\left[x_{1}, \ldots, x_{n}\right]$ um polinômio homogêneo diferente de zero. Para esse, existe unicamente o conceito de grau, seu grau d como polinômio homogêneo, definido por $f(t x)=f\left(t x_{1}, \ldots, t x_{n}\right)=t^{d} f\left(x_{1}, \ldots, x_{n}\right)=t^{d} f(x), t \neq 0$. Polinômios homogêneos admitem uma restrição bem definida em espaços projetivos. A saber, $f$ se restringe a um polinômio em $\mathbb{P}^{n-1}$.

Uma hipersuperfície $V(f) \subset \mathbb{P}^{n-1}(\mathbb{C})$ (ou seja, local dos zeros de $f$ ) de grau d é determinada por um polinômio homogêneo $f\left(x_{1}, \ldots, x_{n}\right) \in \mathbb{C}\left[x_{1}, \ldots, x_{n}\right]$ de grau $d$. Ademais, dois polinômios $f, g \in \mathbb{C}\left[x_{1}, \ldots, x_{n}\right]$ determinam a mesma hipersuperficie $V(f)=V(g)$ se, e somente se, $f=\lambda g$ para algum $\lambda \in \mathbb{C}$.

Não queremos entrar em mais detalhes acerca de geometria algébrica, mas uma hipersuperfície é dita racional se for birracionalmente equivalente a um espaço projetivo. De maneira grosseira, isso quer dizer que existem funções no anel $\mathbb{C}\left[x_{1}, \ldots, x_{n}\right]$ cujas restrições à $V(f)$ são funções racionais (quocientes de polinômios com restrições nos seus graus) cuja imagem é um espaço projetivo, e para essas existe uma inversa também racional.

Dancer-Wang mostram que existe uma estrutura de $S R S(M, g, f)$ compacta, não trivial, e do tipo gradiente; no espaço total de um $\mathbb{C P}^{1}$-fibrado sobre um produto de duas cópias de $\mathcal{F}(3,3)$, onde $\mathcal{F}(3,3)$ consiste na Irrational Clemens-Griffiths threefold (veja [CG72]). Ainda mais, $(M, g, f)$ é uma variedade de Kähler que também é Fano. 


\section{Capítulo 4}

\section{Diagramas Estrela: topologia e geometria}

\subsection{Construindo diagramas estrela}

Considere um fibrado principal $G \hookrightarrow P \rightarrow M$ com ação principal • e cujo espaço total é compacto e conexo. Assuma que $G$ seja também compacto e conexo, e que exista outra ação por $G$ em $P$, a qual denotamos por $\star$, de tal maneira que $\bullet$ e $\star$ comutem. Isso faz com que $P$ seja uma $G \times G$-variedade. Se assumirmos que $\star$ também seja livre, obtemos o seguinte diagrama estrela de fibrados:

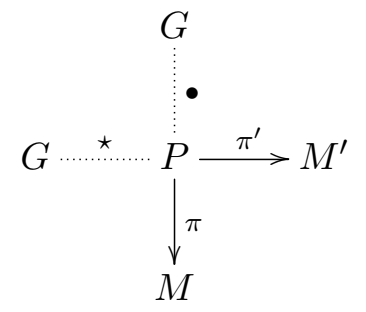

A variedade $M$ no diagrama (4.1) denota o quociente de $P$ pela ação $\bullet$, enquanto $M^{\prime}$ denota o quociente $P$ pela ação $\star$.

Definição 33. O diagrama (4.1) é denominado diagrama estrela. De maneira curta, o denotaremos por $M^{\prime} \leftarrow \mathcal{P} \rightarrow M$.

Como $\bullet$ e $\star$ comutam, $\bullet$ induz uma ação no quociente $M^{\prime}$, e $\star$ induz uma ação em $M$. Denotamos os espaços de órbita segundo tais induzidas ações por $M^{\prime} / \bullet$ e $M / \star$, ou, quando claro o contexto, simplesmente por $M^{\prime} / G$ e $M / G$.

Neste capítulo discutiremos as propriedades geométricas de fibrados satisfazendo um diagrama como o (4.1), além de indicar como realizar diversas variedades exóticas $M^{\prime}$ partindo de um par bem conhecido $(P, M)$. Na seção 5.5 provamos a existência de métricas de curvatura de Ricci positiva e seccional quase não negativa nos exemplos obtidos.

Esse tipo de construção foi inspirada pela realização da esfera exótica de Gromoll-Meyer $\Sigma^{7}$ obtida como $M^{\prime}$ no diagrama (4.1).

Exemplo. Lembre-se que o grupo de Lie Sp(2) é definido por

$$
S p(2)=\left\{\left(\begin{array}{ll}
a & c \\
b & d
\end{array}\right) \in S^{7} \times S^{7} \mid \bar{c} a+\bar{d} b=0\right\},
$$

onde $a, b, c, d \in \mathbb{H}$, são quaternions. A projeção $\pi: S p(2) \rightarrow S^{7}$ de um elemento de $S p(2)$ na sua primeira coluna define um $S^{3}$-fibrado principal munido da ação principal

$$
\left(\begin{array}{ll}
a & c \\
b & d
\end{array}\right) \bar{q}=\left(\begin{array}{ll}
a & c \bar{q} \\
b & d \bar{q}
\end{array}\right)
$$


Em [GM`4] Gromoll-Meyer introduziram a ação *

$$
q\left(\begin{array}{ll}
a & c \\
b & d
\end{array}\right)=\left(\begin{array}{ll}
q a \bar{q} & q c \\
q b \bar{q} & q d
\end{array}\right)
$$

cujo quociente é uma esfera exótica de dimensão 7, concluindo seu célebre resultado sobre a existência de uma esfera exótica com curvatura seccional não-negativa.

A ação correspondente em $S^{7}$ pode ser lida a partir da primeira coluna de (4.3):

$$
q \cdot\left(\begin{array}{l}
x \\
y
\end{array}\right)=\left(\begin{array}{l}
q x \bar{q} \\
q y \bar{q}
\end{array}\right)
$$

As ações dadas por (4.3), (4.4) produzem o seguinte diagrama estrela

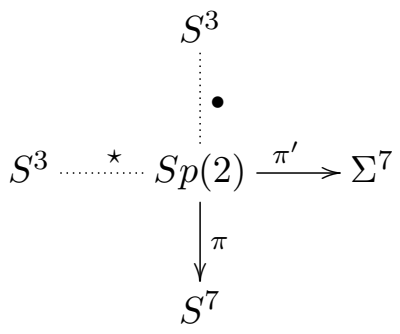

que foi utilizado em [Dur01/ para realizar geometricamente um clutching diffeomorphism $\widehat{b}: S^{6} \rightarrow S^{6}$ para $\Sigma^{7}=S p(2) / \star$.

Neste capítulo provamos que:

Teorema 4.1.1. Sejam $\Sigma^{7}$ e $\Sigma^{8}$ quaisquer variedades fechadas que são homotópicas às esferas padrões de dimensões 7 e 8 , respectivamente; $\Sigma^{10}$ qualquer variedade fechada homotópica à esfera padrão em dimensão 10 e que seja bordo de uma variedade spin; $\Sigma^{4 m+1}, \Sigma^{8 m+5}$ as esferas de Kervaire em dimensões $4 m+1,8 m+5$, respectivamente. Então as seguintes variedades admitem uma realização explícita como no diagrama (4.1) e satisfazem as hipóteses do Teorema C:

(i) $M^{7} \# \Sigma^{7}$, onde $M^{7}$ é qualquer $S^{3}$-fibrado sobre $S^{4}$

(ii) $M^{8} \# \Sigma^{8}$, onde $M^{8}$ é ou um $S^{3}$-fibrado sobre $S^{5}$ ou um $S^{4}$-fibrado sobre $S^{4}$

(iii) $M^{10} \# \Sigma^{10}$, onde:

(a) $M^{10}=M^{8} \times S^{2}$ onde $M^{8}$ é como no item (ii)

(b) $M^{10}$ é qualquer $S^{3}$-fibrado sobre $S^{7}, S^{5}$-fibrado sobre $S^{5}$, ou $S^{6}$-fibrado sobre $S^{4}$

(iv) $M^{4 m+1} \# \Sigma^{4 m+1}$ onde

(a) $S^{2 m} \hookrightarrow M^{4 m+1} \rightarrow S^{2 m+1}$ é o fibrado de esferas associado a um múltiplo ${ }^{1}$ de $O(2 m+1) \hookrightarrow$ $O(2 m+2) \rightarrow S^{2 m+1}$, o fibrado de referenciais de $S^{2 m+1}$

(b) $\mathbb{C P}^{m} \hookrightarrow M^{4 m+1} \rightarrow S^{2 m+1}$ é o $\mathbb{C P}^{m}$-fibrado associado a um múltiplo do fibrado de referenciais unitários $U(m) \hookrightarrow U(m+1) \rightarrow S^{2 m+1}$

(c) $M^{4 m+1}=\frac{U(m+2)}{S U(2) \times U(m)}$

(v) $\left(M^{8 r+k} \times N^{5-k}\right) \# \Sigma^{8 r+5}$ onde $N^{5-k}$ é qualquer variedade com curvatura de Ricci positiva $e$

(a) $S^{4 r+k-1} \hookrightarrow M^{8 r+k} \rightarrow S^{4 r+1}$ é a k-ésima suspensão do fibrado tangente unitário $S^{4 r-1} \hookrightarrow$ $T^{1} S^{4 r+1} \rightarrow S^{4 r+1}$

\footnotetext{
${ }^{1} \mathrm{Ou}$ seja, um fibrado cujas funções de transição $\alpha: S^{n-1} \rightarrow G$ são múltiplas de $\tau_{2 m}: S^{2 m} \rightarrow O(2 m+1)$, $\tau_{m}^{\mathbb{C}}: S^{2 m} \rightarrow U(m)$ ou $\tau_{m}^{\mathbb{H}}: S^{4 m+2} \rightarrow S p(m)$, para $G=O(2 m), U(m+1)$ ou $S p(m)$, as funções de transição do fibrado de referenciais ortonormais e suas reduções, respectivamente.
} 
(b) para $k=1, \mathbb{H} \mathrm{P}^{m} \hookrightarrow M^{8 m+1} \rightarrow S^{4 m+1}$ é o $\mathbb{H}^{m}$-fibrado associado a um múltiplo de $S p(m) \hookrightarrow S p(m+1) \rightarrow S^{4 m+1}$

(c) para $k=0, M=\frac{S p(m+2)}{S p(2) \times S p(m)}$

(d) para $k=1, M=M^{8 m+1}$ é como no item (iv)

Para tanto, comecemos com uma apresentação sistemática sobre como construir diagramas estrela e pullbacks desses. A referência é artigo [CS18b] escrito em colaboração com o Prof. Sperança. Ressaltamos que a contribuição do candidato consiste na descrição das propriedades geométricas desse tipo de fibrado, e não das propriedades topológicas, que fornecem os exemplos. Essas foram essencialmente realizadas pelo orientador. Por isso, na seção 4.3 apenas indicaremos algumas construções explícitas. No que segue, fornecemos a teoria geral para realização de fibrados estrela.

Sejam $M$ uma $G$-variedade (à esquerda) e $\left\{U_{i}\right\}$ uma coleção de abertos de $M$ que seja $G$ invariante. Reduzindo $U_{i}$ se necessário, podemos assumir que $G U_{i}=U_{i}$. Dados dois abertos $G$ invariantes $U_{i}, U_{j}$, denotamos por $U_{i j}:=U_{i} \cap U_{j}$.

Uma coleção $\phi_{i j}: U_{i j} \rightarrow G$ é dita coleção $\star$ se satisfizer a condição de cociclo,

$$
\phi_{i j}(x) \phi_{j k}(x) \phi_{k i}(x)=\phi_{i i}(x), \forall x \in U_{i j},
$$

e também,

$$
\phi_{i j}(g x)=g \phi_{i j}(x) g^{-1}, \forall i, j, \forall g \in G, \forall x \in U_{i j} .
$$

A condição (4.8) garante que a que o mapa adjunto

$$
\begin{gathered}
\widehat{\phi}_{i j}: U_{i j} \rightarrow U_{i j} \\
\widehat{\phi}_{i j}: x \mapsto \phi_{i j}(x) x .
\end{gathered}
$$

seja equivariante (veja a Definição 25).

Dessa maneira, $\hat{\phi}_{i j}$ define um difeomorfismo equivariante sobre $U_{i j}$. De fato, isso segue do seguinte lema:

Lema 5. Sejam $U$ uma $G$-varieade (à esquerda) e $\theta, \theta^{\prime}: U \rightarrow G$ mapas suaves satisfazendo (4.8). Então $\widehat{\theta}$ e $\widehat{\theta^{\prime}}$ são difeomorfismos equivariantes tais que

$$
\widehat{\theta \theta^{\prime}}=\widehat{\theta}^{\prime} \widehat{\theta}
$$

onde $\theta \theta^{\prime}(x):=\theta(x) \theta^{\prime}(x), \forall x \in U$.

Demonstração. Seja $x \in U$. Então,

$$
\begin{aligned}
\widehat{\theta^{\prime} \theta}(x) & :=\left(\theta^{\prime} \theta\right)(x) x \\
& =\theta^{\prime}(\theta(x) x) \theta^{\prime}(x) x \\
& =\theta(x) \theta^{\prime}(x) \theta(x)^{-1} \theta(x) x \\
& =\theta(x) \theta^{\prime}(x) x \\
& =\widehat{\theta \theta^{\prime}}(x) .
\end{aligned}
$$

Em particular, se tomarmos $\theta^{\prime}=\theta^{-1}$, teremos $\widehat{\theta^{-1}}=(\widehat{\theta})^{-1}$.

Por fim, verificamos que $\widehat{\theta}$ é $G$-equivariante calculando

$$
\begin{aligned}
\widehat{\theta}(g x) & :=\theta(g x) g x \\
& =g \theta(x) g^{-1} g x \\
& =g \theta(x) .
\end{aligned}
$$

o que conclui a demonstração. 
Seja $\left\{\phi_{i j}: U_{i j} \rightarrow G\right\}$ uma coleção $\star$. Definimos

$$
\bigcup_{\widehat{\phi}_{i j}} U_{i}
$$

como sendo o espaço quociente associado à seguinte relação de equivalência:

$$
x \in U_{i j} \sim \widehat{\phi}_{i j} x \in U_{i j}
$$

Ou seja, dados $x, y \in M$, dizemos que $x$ é equivalente a $y$ se $\exists i, j$ para os quais $x, y \in U_{i j}$ e ainda, $\phi_{i j}(x) x=y$.

O alicérce da construção de diagramas estrela reside no próximo teorema. Assumiremos a existência de uma coleção $\star$ na $G$-variedade $M$ que é base em um fibrado principal $\pi: \mathcal{P} \rightarrow M$. Nesse caso, dizemos que $\pi$ está associada à coleção $\star$ definida via $M$.

Teorema 4.1.2. Seja $\pi: \mathcal{P} \rightarrow M$ o fibrado principal associado a uma coleção $\star$ dada por $\left\{\phi_{i j}:\right.$ $\left.U_{i j} \rightarrow G\right\}$. Então $\mathcal{P}$ admite uma nova ação, denotada por $\star$, tal que

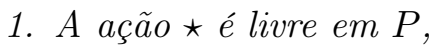

2. O quociente $P / \star$ é uma G-variedade equivariantemente difeomorfa a

$$
M^{\prime}:=\bigcup_{\widehat{\phi}_{i j}} U_{i},
$$

3. O diagrama estrela 4.1 obtido dessa construção é tal que se $(G \times G)_{p}$ denota o grupo de isotropia em $p$ com respeito à a ação por justaposição

$$
(r, s) p:=r p s^{-1}
$$

onde $G \times\{e\}$ representa a ação $\star,\{e\} \times G$ representa a ação principal, $e$ "e" denota o elemento neutro de $G$, então existe $g \in G$ tal que

$$
(G \times G)_{p}=\left\{\left(h, g h g^{-1}\right): g \in G_{\pi(p)}\right\} .
$$

Demonstração. Seja $\left\{\phi_{i j}: U_{i j} \rightarrow G\right\}$ a coleção $\star$ associada a $\pi: \mathcal{P} \rightarrow M$ fornecida pela hipótese. Em particular, conseguimos trivializar $\mathcal{P}$ de maneira que $\left.P\right|_{\pi^{-1}\left(U_{i}\right)} \cong U_{i} \times G$. Assim, definimos a ação $\star$ como

$$
r(x, g):=\left(x r^{-1}, r g\right), \forall r \in G
$$

que é claramente livre.

Lembramos que $\mathcal{P}$ pode ser recuperado via o espaço quociente

$$
\bigcup_{\phi_{i j}} U_{i} \times G=\mathcal{P}
$$

onde os elementos $(x, g),(y, h) \in \bigcup_{i} U_{i} \times G$ são equivalente se, e somente se, existirem $i, j$ tais que

$$
x=y \in U_{i j}, g \phi_{i j}(x)=h .
$$

Assim, para verificar a boa definição global, note que pela equação (25), dado $x \in U_{i j}$ temos que

$$
\left(q x, g q^{-1} \phi_{i j}(q x)\right)=\left(q x, g \phi_{i j}(x) q^{-1}\right), x \in U_{i j}, q, g \in G .
$$

Lembrando que pelo Lema 5 as funções adjuntas $\widehat{\phi}_{i j}$ são difeomorfismos equivariantes, definimos 
a projeção

$$
\pi^{\prime}: \mathcal{P} \rightarrow M^{\prime}
$$

a partir de uma trivialização local

$$
\pi^{\prime}:(x, g) \in U_{i} \times G \mapsto g x .
$$

Note que $\pi^{\prime}$ fica bem definida já que

$$
\begin{aligned}
\pi^{\prime}\left(x, g \phi_{i j}(x)\right) & =g \phi_{i j}(x) x \\
& =g \widehat{\phi}_{i j}(x) \\
& =\widehat{\phi}_{i j}(g x) \\
& =\phi_{i j}(g x) g x \\
& =\phi_{i j}\left(\pi^{\prime}(x, g)\right) \pi^{\prime}(x, g) \\
& =\widehat{\phi}_{i j}\left(\pi^{\prime}(x, g)\right) .
\end{aligned}
$$

Ou seja, $\pi^{\prime}\left(x, g \phi_{i j}(x)\right)$ e $\pi^{\prime}(x, g)$ definem a mesma classe de equivalência em $M^{\prime}$.

Mais ainda, $\pi^{\prime}(r p)=\pi^{\prime}(p)$, e daí $\pi^{\prime}$ é uma submersão cujas fibras são as órbitas da ação $\star$. Em particular, o mapa

$$
\iota_{i}(x):=(x, e) \text {, onde } e \text { denota o elemento neutro de } G \text {. }
$$

define uma seção local tanto para $\pi$ quanto para $\pi^{\prime}$. Portanto, $U_{i} \times G / \star$ e $U_{i}$ são abertos equivariantemente difeomorfos.

Por fim, a afirmação sobre o grupo de isotropia $(G \times G) p$ segue imediatamente do fato que numa trivialização local para $\mathcal{P}$

$$
(r, s)(x, g):=\left(r x, s g r^{-1}\right), x \in U_{i}, r, s, g \in G .
$$

Precisamente, $(r, s)(x, g)=(x, g)$ se, e somente se, $r \in G_{x}$ e $s g=r$. Logo,

$$
(G \times G)_{p}=\left\{(s g, s): s g \in G_{x} \forall g \in G\right\} .
$$

Por fim, dado $h \in G_{x}$, então para qualquer $g \in G, g h g^{-1}$ é tal que $g h g^{-1} g=g h \in G_{x}$, o que conclui o afirmado.

Um método prolífico para construção de exemplos consiste em tomar pullbacks de $\mathcal{P}$ em um diagrama estrela respeitando algumas propriedades. Terminamos esta seção elucidando esse fato.

Sejam $M, N G$-variedades e $f: N \rightarrow M$ uma aplicação suave e equivariante. Seja $\left\{\phi_{i j}: U_{i j} \rightarrow\right.$ $G\}$ uma coleção $\star$ associada ao fibrado $\pi: \mathcal{P} \rightarrow M$. Recorde que o fibrado pullback por $f$ sobre $N$ é definido pelo espaço total

$$
f^{*}(\mathcal{P}):=\{N \times \mathcal{P}: f(n)=\pi(p), \forall n \in N, \forall p \in \mathcal{P}\},
$$

com projeção $\pi_{f}(n, p):=n$. Assim, a ação principal induzida em $f^{*}(\mathcal{P})$ é definida por

$$
(n, p) s^{-1}:=(n, s p)
$$

enquanto ação induzida em $f^{*}(\mathcal{P})$ é definida por

$$
r(n, p):=\left(r n, p r^{-1}\right)
$$

Proposição 4.1.3. Seja $f: N \rightarrow M$ uma função suave e G-equivariante e $M^{\prime} \leftarrow \mathcal{P} \rightarrow M$ um fibrado estrela obtido por uma coleção $\star$ dada por $\left\{\phi_{i j}: U_{i j} \rightarrow G\right\}$. Então, 
1. $\pi_{f}$ consiste em um fibrado que é equivariantemente difeomorfo ao fibrado estrela obtido pela coleção $\star$ dada por $\left\{\phi_{i j} \circ f: f^{-1}\left(U_{i j}\right) \rightarrow G\right\}$.

2. O quociente $f^{*}(\mathcal{P}) / \star$ é equivariantemente difeomorfo a

$$
N^{\prime}=\bigcup_{\widehat{\phi_{i j} \circ f}} f^{-1}\left(U_{i}\right)
$$

3. Fica bem definido um mapa $f^{\prime}: N^{\prime} \rightarrow M^{\prime}$ tal que $\left.f^{\prime}\right|_{f^{-1}\left(U_{i}\right)}=\left.f\right|_{f^{-1}\left(U_{i}\right)}$.

Demonstração. Veja [CS18b, Proposition 2.4, p. 11-12]

\subsection{A geometria dos diagramas estrela}

Esta seção tem como objetivo comparar as geometrias horizontais de $M$ e $M^{\prime}$ em um fibrado estrela.

Seja $M^{\prime} \leftarrow \mathcal{P} \rightarrow M$ um fibrado estrela. Denote $\bullet$ a ação principal em $\mathcal{P}$. Lembrando que dado que $\bullet$ e $\star$ comutam, $\star$ induz uma ação por $G$ no quociente $M$ e $\bullet$ induz uma ação por $G$ em $M^{\prime}$. Assim, veremos $M$ e $M^{\prime}$ como $G$-variedades associadas a tais ações.

Um método eficiente para comparar as geometrias de $M$ e $M^{\prime}$ consiste em construir uma métrica $G \times G$-invariante em $\mathcal{P}$ de modo que as projeções $\pi$ e $\pi^{\prime}$ induzam métricas de submersão riemanniana em $M$ e $M^{\prime}$, respectivamente. Em particular, se começarmos com uma métrica $G \times G$-invariante em $\mathcal{P}$, se $g_{M}$ denota a métrica de submersão riemanniana induzida por $\pi$ em $M$, provamos o seguinte teorema:

Teorema 4.2.1. Seja $M^{\prime} \leftarrow \mathcal{P} \rightarrow M$ um fibrado estrela. Então, existe uma métrica •-invariante em $M^{\prime}$ tal que $M / G$ e $M^{\prime} / G$ são isométricos como espaços métricos.

Para tanto, seja

$$
\mathcal{H}^{\prime \prime}:=\{X \in \Gamma(T P): X \perp T(G \times G)\}
$$

isto é, $\mathcal{H}^{\prime \prime}$ denota o espaço horizontal segundo a ação $(r, s) p \mapsto r p s^{-1}$. Se $M$ e $M^{\prime}$ estão munidas de métricas riemannianas de submersão, então as restrições de $d \pi$ e $d \pi^{\prime}$ a $\mathcal{H}^{\prime \prime}$ induzem isometrias entre $\mathcal{H}^{\prime \prime}$ e $\mathcal{H} \subset T M, \mathcal{H}^{\prime} \subset T M^{\prime}$, respectivamente.

Se começarmos com uma métrica $g$ em $\mathcal{P}$, uma maneira natural (assumindo que $G$ seja compacto) de obter uma métrica $G \times G$-invariante consiste em tomar o average (Definição 19) de $g$ sobre $G$. No entanto, tomaremos outra abordagem.

Lembre-se que a ação de $G$ em $\mathcal{P}$ induz ações em $T P$ (pela diferencial da representação). Abusamos notação de forma que a ação induzida em $T P$ seja escrita como $g X, g \in G, X \in \Gamma(T P)$.

Definição 34. Uma 1-forma de conexão $\omega_{0}: \Gamma(T P) \rightarrow \mathfrak{g}$ em um fibrado principal $\pi: G \hookrightarrow \mathcal{P} \rightarrow M$ consiste de uma aplicação suave tal que

1. $\omega_{0}\left(U^{*}\right)=U, \forall U \in \mathfrak{g}$, onde $U^{*}$ denota o campo de ação associado a $U$,

2. $\left(\omega_{0}\right)_{p g}(X g)=\operatorname{Ad}_{g^{-1}}\left(\omega_{0}\right)_{p}(X)$.

Dada uma 1-forma de conexão $\omega: \Gamma(T \mathcal{P}) \rightarrow \mathfrak{g}$ e uma métrica $G$-invariante $g_{M}$ em $M$, induzimos em $\mathcal{P}$ a métrica de Kaluza-Klein definida por

$$
\langle X, Y\rangle:=g_{M}(d \pi X, d \pi Y)+Q(\omega(X), \omega(Y)),
$$

onde $Q$ é uma métrica bi-invariante em $G$. Se $g_{M}$ é $G$-invariante e $\omega$ satisfaz $\omega_{g p}(g X)=\omega_{p}(X), \forall g \in$ $G, X \in \Gamma(T P)$, então $\langle\cdot, \cdot\rangle$ é $G \times G$-invariante. 
Proposição 4.2.2. Existe uma 1-forma de conexão $\omega: \Gamma(T \mathcal{P}) \rightarrow \mathfrak{g}$ tal que para todo $X \in \Gamma(T \mathcal{P})$ e todo $r \in G$

$$
\omega_{r p}(r X)=\omega_{p}(X)
$$

Mais ainda, $\langle\cdot, \cdot\rangle$ definida por (4.11) é $G \times G$-invariante.

Demonstração. Após tomar o average (Definição 19) de $\omega_{0}$,

$$
\omega_{p}(X):=\int_{G}\left(\omega_{0}\right)_{p r}(r X) d r
$$

concluímos imediatamente o resultado uma vez que $\bullet$ e $\star$ comutam.

Doravante fixemos uma métrica de Kaluza-Klein $G \times G$-invariante em $\mathcal{P}$. Como a ação de $G \times G$ em $\mathcal{P}$ é por isometrias, existe única métrica de submersão riemanniana em $M^{\prime}$. Ademais, essa é $G$-invariante. Para demonstrar o Teorema 4.2.1 apenas notamos que se $x=\pi(p)$ e $x^{\prime}=\pi^{\prime}(p)$, então $\pi^{-1}(G x)=(G \times G) p=\pi^{\prime-1}(x)$, Assim, fica definida uma bijeção

$$
i: M / G \rightarrow M^{\prime} / G
$$

Em particular, $i$ é uma isometria. De fato, se $\gamma:[0,1] \rightarrow \mathcal{P}$ é uma geodésica $\mathcal{H}^{\prime \prime}$ horizontal, então $\pi \circ \gamma$ e $\pi^{\prime} \circ \gamma$ são geodésicas horizontais às órbitas de $G$ em $M$ e $M^{\prime}$, respectivamente. Portanto, dados $p_{1}, p_{2} \in \mathcal{P}$, se $\gamma$ denota o menor segmento geodésico ligando as órbitas $(G \times G) p_{1}$ e $(G \times G) p_{2}$, então $\pi \circ \gamma$ e $\pi^{\prime} \circ \gamma$ possuem o mesmo comprimento, e são as geodésicas minimizantes entre $G \pi\left(p_{1}\right)$ e $G \pi\left(p_{2}\right) ; G \pi^{\prime}\left(p_{1}\right)$ e $G \pi^{\prime}\left(p_{2}\right)$; respectivamente. Isso conclui a demonstração do Teorema 4.2.1.

Teorema 4.2.3. Seja $M^{\prime} \leftarrow \mathcal{P} \rightarrow M$ um fibrado estrela. Então as órbitas da ação de $G$ em $M$ possuem grupo fundamental finito se, e somente se, as órbitas da ação de $G$ em $M^{\prime}$ também possuírem.

Demonstração. Pelo Teorema 3.1.13, se uma órbita principal da ação por $G$ em $M$ possui grupo fundamental finito, então todas as $G$-órbitas em $M$ possuem. Provemos então que as órbitas por $G$ em $M^{\prime}$ são homeomorfas às órbitas por $G$ em $M$. Denote por $\Delta G_{x^{\prime}}=\left\{(q, q) \in G: q \in G_{x^{\prime}}\right\}$. Pelo item 3 do Teorema 4.1.2, dado $x^{\prime} \in M^{\prime}$ existe $p \in\left(\pi^{\prime}\right)^{-1}(x)$ tal que $(G \times G)_{p}=\Delta G_{x^{\prime}}$. Seja $x=\pi(p)$. Então,

$$
G x \cong(G \times G) p /(\{e\} \times G) \cong(G \times G) p /(G \times\{e\}) \cong G x^{\prime} .
$$

Como os papéis de $M$ e $M^{\prime}$ podem ser trocados, o resultado segue.

Demonstração do Teorema 4.1.1. Uma vez construídas as variedades do Teorema 4.1.1, esse resultado segue como corolário dos Teoremas 4.2.1 e 4.2.3.

Passemos às construções.

\subsection{Realizações explícitas de variedades exóticas via diagramas es- trela (um esboço)}

A construção dos exemplos no Teorema 4.1.1 está relacionada à presença de simetrias e de realizações explícitas de esferas exóticas. Fixe as seguintes representações:

$\left(\rho_{7}\right) n=7, G=S^{3}: \rho=\rho_{1} \oplus \rho_{1} \oplus \rho_{0}$, onde $\rho_{1}: S^{3} \rightarrow O(3)$ é a representação induzida pelo recobrimento duplo $S^{3} \rightarrow S O(3)$ e $\rho_{0}: S^{3} \rightarrow S O(1)$ é a representação trivial;

$\left(\rho_{8}\right) n=8, G=S^{3}: \rho=\rho_{\frac{1}{2}} \oplus \rho_{1} \oplus \rho_{0}$, onde $\rho_{\frac{1}{2}}: S^{3} \rightarrow O(4)$ é a representação induzida pela multiplicação à direita (ou à esquerda) por um quaternion; 
$\left(\rho_{10}\right) n=10, G=S^{3}: \rho=\rho_{\frac{1}{2}} \oplus \rho_{1} \oplus 3 \rho_{0}$ ou $\rho=\rho_{1} \oplus \rho_{1} \oplus \rho_{1} \oplus \rho_{0} ;$

$\left(\rho_{4 m+1}\right) n=4 m+1, G=U(m): \rho=\rho_{U(m)} \oplus \rho_{U(m)} \oplus \rho_{0}$ onde $\rho_{U(m)}: U(m) \rightarrow O(2 m)$ é a representação canônica;

$\left(\rho_{8 m+5}\right) n=8 m+5, G=S p(m): \rho=\rho_{S p(m)} \oplus \rho_{S p(m)} \oplus 5 \rho_{0}$ onde $\rho_{S p(m)}: S p(m) \rightarrow O(4 m)$ é a representação canônica.

Em [Spe16b], são construídos diagramas como (1.1) com $M=S^{n}$ e $M^{\prime}=\Sigma^{7}, \Sigma^{8}, \Sigma^{10}, \Sigma^{4 m+1} \mathrm{e}$ $\Sigma^{8 m+5}$. Nessas construções, cada $\Sigma^{n}$ possui uma $G$-ação com um ponto fixo cuja representação de isotropia é precisamente $\left(\rho_{n}\right)$.

Dada uma métrica $G$-invariante em $M$, relembramos que a distância entre duas órbitas induz uma métrica que torna $M / G$ um espaço métrico (a métrica de distância orbital). Lembramos também que as $G$-órbitas possuem dimensão constante em um aberto denso $M^{r e g} \subseteq M$. Recorde também que $M^{r e g} / G$ possui uma estrutura de variedade suave que torna a aplicação no quociente $M^{r e g} \rightarrow M^{r e g} / G$ uma submersão riemanniana. Denote por $\operatorname{Ric}_{M^{r e g} / G}$ a curvatura de Ricci com respeito à métrica induzida em $M^{r e g} / G$.

Teorema 4.3.1. Seja $M^{n}$ uma $G$-variedade e assuma que $M$ tenha um ponto fixo com representação de isotropia dada por $\left(\rho_{n}\right)$. Então:

1. Se as órbitas em $M^{\text {reg }}$ possuem grupo fundamental finito e $M$ possui uma métrica $G$-invariante satisfazendo $\operatorname{Ric}_{M^{r e g} / G} \geq 1$, então $M^{n} \# \Sigma^{n}$ possui uma métrica com curvatura de Ricci positiva;

2. Se $M$ admite uma sequência de métrias $G$-invariantes tais que $M^{\text {reg }} / G$ possua curvatura seccional quase não-negativa, então $M^{n} \# \Sigma^{n}$ também possui uma sequência com tal propriedade;

3. Se $M$ possui uma sequência de métricas satisfazendo as hipóteses em (1) e (2) simultanemanete, então $M^{n} \# \Sigma^{n}$ admite uma sequência de métricas satisfazendo as teses de (1) e (2) simultaneamente;

Para a demonstração do Teorema 4.3.1, construíremos um diagrama como (1.1) com $G$ sendo um grupo de Lie compacto e $M^{\prime}$ difeormorfa a $M^{n} \# \Sigma^{n}$. De acordo com o Teorema 4.2.1, dada uma métrica $G$-invariante em $M$, existe uma métrica $G$-invariante em $M^{\prime}$ tal que $M^{\prime} / G$ é isométrico a $M / G$. O Teorema 4.3.1 seguirá portanto da discussão nas seções anteriores e das construções na seção 5.5.

Aproveitamos para observar que a soma conexa $M^{n} \# \Sigma^{n}$ nem sempre resulta em uma nova variedade. Seguindo de Sapio [dS69a, dS69b], concluímos que $M^{n} \# \Sigma^{n} \cong M^{n}$ se $M^{n}$ é: o $S U(2)$ fibrado principal não trivial sobre $S^{5}$; o $S U(2)$-fibrado principal sobre $S^{7}$ que corresponde ao gerador de $\pi_{6} S U(2) \cong \mathbb{Z}_{12}$; o fibrado tangente unitário $T_{1} S^{2 m+1}$. Por outro lado, $M^{n} \# \Sigma^{n} ¥ M^{n}$ quando $M^{n}$ é: qualquer produto de esferas padrões; qualquer 4-fibrado de esferas sobre $S^{4} ; \Sigma^{8} \times S^{2} ;$ o $S U(2)$ fibrado principal correspondente à três vezes um gerador em $\pi_{6} S U(2)$ (lembre-se de [Spe16b, p. 3190] e [Bre72, Section V.8] que, de acordo com a notação de de Sapio, $\Sigma^{8} \in \sigma\left(\pi_{3} S O(4), \pi_{4} S O(3)\right), \Sigma^{10}$ é um elemento de ordem $3 \mathrm{em} \sigma\left(\pi_{3} S O(6), \pi_{6} S O(3)\right)$ e $\Sigma^{4 m+1}=\sigma\left(\tau_{2 m}, \tau_{2 m}\right)$, onde $\tau_{n}: S^{n} \rightarrow S O(n+1)$ é uma função de transição para o fibrado tangente de $S^{n+1}$; onde $\sigma$ denota classes de homotopia).

Para a construção dos diagramas necessários procederemos via pull-backs (veja a Proposição 4.1.3) para realizar os seguintes diagramas estrela $M \leftarrow f^{*} \mathcal{P} \rightarrow M \# \Sigma^{n}$.

Seja $M$ uma $G$-variedade com um ponto fixo $p \in M$. Assuma que $G$ seja compacto e que $M$ esteja munida com uma métrica riemanniana que seja $G$-invariante. Então, a diferencial da ação em $p$ induz um morfimo $\rho: G \rightarrow O\left(T_{p} M\right)$ chamado de representação de isotropia de $G$ em $p$.

Por outro lado, consideramos $S^{n} \subset \mathbb{R} \times T_{p} M$ como sendo a $G$-variedade munida da ação $g$. $(\lambda, x)=(\lambda, \rho(g) x)$. Note que $e_{0}=(1,0)^{T}$ é um ponto fixo. Sejam $D_{ \pm}=\left\{(\lambda, x) \in S^{n} \mid \pm \lambda \geq 0\right\}$ e $S^{n-1}=D_{+} \cap D_{-}$, e observe que $D_{+}, D_{-}, S^{n-1}$ são subconjuntos $G$-invariantes. Se $\phi: S^{n-1} \rightarrow G$ 
satisfaz a condição de equivariância descrita na equação (4.8), podemos construrir o $G$ - $G$-fibrado principal $\mathcal{P} \rightarrow S^{n}$

$$
\mathcal{P}=D_{+} \times G \bigcup_{f_{\phi}} D_{-} \times G
$$

onde $f_{\phi}(x, g)=(x, g \phi(x))$ e a ação $\star$ é definida por $r(x, g)=\left(r \cdot x, g r^{-1}\right)$. Usando a decomposição (4.12) e o Teorema 4.1.2 identificamos $\left(S^{n}\right)^{\prime} \operatorname{com}\left(S^{n}\right)^{\prime}=D^{n} \cup_{\hat{\phi}} D^{n}$.

A próxima etapa consiste em tomar o pull-back de $\mathcal{P}$ para $M$. Seja $D_{\epsilon}$ um disco de raio $\epsilon$ ao redor do ponto fixo $p$ tal que $\left.\exp _{p}\right|_{D_{\epsilon}}$ seja um difeomorfismo. Definimos $f: M \rightarrow S^{n}$ por

$$
f(x)= \begin{cases}\exp _{e_{0}}\left(\pi \phi\left(\frac{\left|\exp _{p}^{-1}(x)\right|}{\epsilon}\right) \frac{\exp _{p}^{-1}(x)}{\left|\exp _{p}^{-1}(x)\right|}\right), & x \in D_{\epsilon} \\ -e_{0}, & x \notin D_{\epsilon} .\end{cases}
$$

Como $D_{ \pm}$são subconjuntos trivializantes para $\mathcal{P}$, pela Proposição 4.1 .3 concluímos

$$
f^{*} \mathcal{P}=f^{-1}\left(D_{+}\right) \times G \bigcup_{f_{\phi \circ f}} f^{-1}\left(D_{-}\right) \times G=\left(M-D_{\epsilon / 2}\right) \times G \bigcup_{f_{\phi}} D_{\epsilon / 2} \times G,
$$

onde $D_{\epsilon / 2}$ é um disco de raio $\epsilon / 2$ ao redor de $p$; e identificamos $S^{n-1}$ com $\partial D_{\epsilon / 2}$. Portanto,

$$
M^{\prime}=\left(M-D_{\epsilon / 2}\right) \bigcup_{\hat{\phi}} D_{\epsilon / 2}
$$

$M^{\prime}$ é então difeomorfa a $M \#\left(S^{n}\right)^{\prime}$. Em suma, acabamos de provar o seguinte:

Teorema 4.3.2. Seja $M^{n}$ uma $G$-variedade riemanniana e $p \in M$ um ponto fixo com representação de isotropia $\rho: G \rightarrow O(n)$. Então, dado um mapa $\phi: S^{n-1} \rightarrow G$ satisfazendo

$$
\phi(\rho(g) x)=g \phi(x) g^{-1},
$$

existe um G-G-fibrado $M \leftarrow \mathcal{P} \rightarrow M \# \Sigma^{n}$, onde $\Sigma^{n}=D^{n} \cup_{\hat{\phi}} D^{n}$.

Os Teorema 1 e 2 em [Spe16b] fornecem funções $\phi: S^{n-1} \rightarrow G$ realizando as esferas

$$
\Sigma^{7}, \Sigma^{8}, \Sigma^{10}, \Sigma^{4 k+1}, \Sigma^{8 k+5}
$$

no Teorema 4.1.1. Fórmulas explícitas para $\phi \operatorname{nos} \operatorname{casos} \Sigma^{7}, \Sigma^{8}$ e $\Sigma^{10}$ são apresentadas em [Dur01] e [Spe16b, Theorem 4.6].

Observe que as representação $\left(\rho_{n}\right)$ são relacionadas a pontos fixos das $S^{3}$-variedades $\Sigma^{7}, \Sigma^{8}, \Sigma^{10}$; e a pontos fixos das $U(m)$-variedades $\Sigma^{4 m+1}$ e $S p(m)$-variedades $\Sigma^{8 m+5}$. As realizações do Teorema 4.1.1 são conseguidas observando que cada uma dessas variedades possui uma $G$-ação com ponto fixo tendo como representação de isotropia $\left(\rho_{n}\right)$. O procedimento a ser seguido consiste em explorar a reducibilidade das representações $\left(\rho_{7}\right)-\left(\rho_{8 m+5}\right)$ para decompor os exemplos em produtos locais, utilizando espaços homogêneos para representar cada parte dessa decomposição. De maneira geral, dado um espaço homogêneo $H / K$ o par $\left(H / K, \rho^{\prime}\right)$ deve ser interpretado como a $G$-variedade $H / K$ com ação $\rho^{\prime}(G) \subset K$. Dessa maneira, $\rho^{\prime}$ é uma subrepresentação da representação de isotropia de $H / K$ no ponto base $K \in H / K$. Para o que segue, $\left(N, \rho^{\prime}\right)$ denotará uma variedade que possui um ponto fixo com representação de isotropia $\rho^{\prime}$. As realizações são obtidas por esse meio ao observar o seguinte as seguintes $G$-variedades possuem pontos fixos com representação $\left(\rho_{n}\right)$.

$$
\begin{aligned}
\left(\rho_{7}\right) \quad \text { 1. } & \left(S^{3}, \rho_{1}\right) \times\left(S^{4}, \rho_{1} \oplus \rho_{0}\right) \\
& \text { 2. }\left(S^{3}, \rho_{1}\right) \times\left(S^{3}, \rho_{1}\right) \times\left(N^{1}, \rho_{0}\right) \\
& \text { 3. }\left(S^{6}, \rho_{1} \oplus \rho_{1}\right) \times\left(N^{1}, \rho_{0}\right) \\
\left(\rho_{8}\right) \quad \text { 4. } & \left(S^{3}, \rho_{1}\right) \times\left(S^{5}, \rho_{\frac{1}{2}} \oplus \rho_{0}\right)
\end{aligned}
$$


5. $\left(S^{3}, \rho_{1}\right) \times\left(S^{4}, \rho_{\frac{1}{2}}\right) \times\left(N^{1}, \rho_{0}\right)$

6. $\left(S^{7}, \rho_{\frac{1}{2}} \oplus \rho_{1}\right) \times\left(N^{1}, \rho_{0}\right)$

$\left(\rho_{10}\right) \quad$ 7. $\left(N^{8}, \rho_{\frac{1}{2}} \oplus \rho_{1} \oplus \rho_{0}\right) \times\left(N^{2}, 2 \rho_{0}\right)$

$\left(\rho_{4 m+1}\right) \quad$ 8. $\left(S^{2 m}, \rho_{U(m)}\right) \times\left(S^{2 m+1}, \rho_{U(m)} \oplus \rho_{0}\right)$

9. $\left(S^{4 m}, \rho_{U(m)} \oplus \rho_{U(m)}\right) \times\left(N^{1}, \rho_{0}\right)$

10. $\left(\mathbb{C} P^{m}, \rho_{U(m)}\right) \times\left(S^{2 m+1}, \rho_{U(m)} \oplus \rho_{0}\right)$

11. $\left(\mathbb{C} P^{m}, \rho_{U(m)}\right) \times\left(S^{2 m}, \rho_{U(m)}\right) \times\left(N^{1}, \rho_{0}\right)$

12. $\left(\mathbb{C} P^{m}, \rho_{U(m)}\right) \times\left(\mathbb{C} P^{m}, \rho_{U(m)}\right) \times\left(N^{1}, \rho_{0}\right)$

13. $\left(\frac{U(m+2)}{S U(2) \times U(m)}, \rho_{U(m)} \oplus \rho_{U(m)} \oplus \rho_{0}\right)$

14. $\left(\frac{U(m+2)}{U(2) \times U(m)}, \rho_{U(m)} \oplus \rho_{U(m)}\right) \times\left(N^{1}, \rho_{0}\right)$

$\left(\rho_{8 m+5}\right)$ 15. $\left(S^{4 m}, \rho_{S p(m)}\right) \times\left(S^{4 m+5}, \rho_{S p(m)} \oplus 5 \rho_{0}\right)$

16. $\left(S^{8 m}, \rho_{S p(m)} \oplus \rho_{S p(m)}\right) \times\left(N^{5}, 5 \rho_{0}\right)$

17. $\left(\mathbb{H} P^{m}, \rho_{S p(m)}\right) \times\left(S^{4 m+l}, \rho_{S p(m)} \oplus l \rho_{0}\right) \times\left(N^{5-l},(5-l) \rho_{0}\right)$, for $0 \leq l \leq 5$

18. $\left(\mathbb{H} P^{m}, \rho_{S p(m)}\right) \times\left(\mathbb{H} P^{m}, \rho_{S p(m)}\right) \times\left(N^{5}, 5 \rho_{0}\right)$

19. $\left(\frac{S p(m+2)}{S p(2) \times S p(m)}, \rho_{S p(m)} \oplus \rho_{S p(m)}\right) \times\left(N^{5}, 5 \rho_{0}\right)$. 


\section{Capítulo 5}

\section{Deformações de Cheeger}

Neste capítulo introduzimos as chamadas deformações de Cheeger (seção 5.1), que se aplicam à variedades munidas de ações isométricas por um grupo de Lie compacto e conexo (ou seja, à $G$-variedades riemannianas), e fornecemos diversas aplicações dessa ferramenta. Precisamente, começamos por definir os tensores fundamentais associados a essas deformações, junto de fórmulas computáveis para curvaturas seccional, de Ricci e escalar de uma métrica deformada. Forneceremos uma abordagem sistemática, inspirada na apresentada em [M8̈7].

Na Seção 5.2 exploraremos o comportamento dos tensores introduzidos na Seção 5.1 em pontos singulares, apresentando respostas definitivas no que se refere à condições necessárias e suficientes para que as deformações de Cheeger sejam efetivas (como única ferramenta) em garantir existência de métricas de curvatura de Ricci positiva em variedades satisfazendo as hipóteses do Teorema C. Em particular, construímos os primeiros exemplos (globais) para os quais as deformações de Cheeger não são efetivas. Como consequências das técnica desenvolvidas, apresentamos um refinamento para o Teorema de Lawson-Yau sobre existência de métricas de curvatura escalar positiva em $G$ variedades sob a hipótese que $G$ seja não-abeliano (Teorema 5.3.1); além de uma demonstração simplificada para o Teorema C (Teorema 5.4.1).

Na seção 5.5 utilizamos as deformações de Cheeger em fibrados estrela e concluímos a demonstração do Teorema A.

Na Seção 5.6 generalizamos o conceito de deformações de Cheeger para o contexto de fibrados com grupo de estrutura compacto, seguindo um desenvolvimento sistemático análogo ao apresentado na subseção 5.1. Por fim, na Seção 5.7 apresentamos condições necessárias e suficientes para existência de curvatura seccional positiva em $G$-variedades riemannianas (Teorema 5.7.2).

\subsection{Deformações de Cheeger clássicas}

Na presente seção discutiremos o conceito de deformações de Cheeger clássicas. Tais deformações foram originalmente introduzidas por Cheeger em [Che73], sendo motivadas pelas métricas de Berger em esferas, com o intuito de construir novos exemplos de variedades com curvatura seccional nãonegativa.

As deformações de Cheeger, aplicadas à $G$-variedades riemannianas (Definição 3.1.1), consistem em deformações que curvam uma métrica invariante nas direções das órbitas. Existem reparametrizações dos 2-planos $\{X, Y\} \in \mathrm{Gr}_{2}(T M)$ de tal maneira que a curvatura seccional da métrica deformada é não decrescente. Assim, se a métrica original tem curvatura seccional não negativa, ou positiva, então nos planos reparametrizados essa propriedade é mantida.

Em 1987, Michael Müter estudou em sua tese de doutorado as construções de Cheeger e suas propriedades de curvatura de maneira detalhada (veja [M $8 \ddot{8} 7]$ ). Esse foi o primeiro trabalho que fornece uma descrição sistemática dessas deformações, além de fórmulas bem computáveis e gerais (veja por exemplo o Teorema 5.1.1). Sua abordagem é muito clara e precisa, e será a grande referência utilizada nesta seção. Entretanto, como de difícil acesso, uma vez que a tese foi escrita em alemão, dado que em 2009 Wolfgang Ziller escreveu um sumário [Zil] que destaca as principais fórmulas 
e resultado desse material, sempre que necessário indicaremos essa referência para consulta, ao invés da tese original. Nas próximas linhas apresentaremos os principais tensores associado a essas deformações.

Seja $(M, g)$ uma variedade riemanniana e seja $G$ um grupo de Lie compacto e conexo munido de uma métrica bi-invariante $Q$, agindo em $(M, g)$ por isometrias (veja a Definição 7 ).

Considere o produto $M \times G$ munido da ação livre

$$
r \cdot(p, g):=(r \cdot p, r g)
$$

Nota-se que o quociente de $M \times G$ por essa ação é difeomorfo a $M$ segundo a aplicação quociente definida por $\sigma(p, g)=g^{-1} \cdot p$. Desse modo, se considerarmos a métrica $g \times \frac{1}{t} Q$ em $M \times G, t>0$, conseguimos induzir uma família a um parâmetro de métricas riemannianas $g_{t}$ em $M$ através da submersão $\sigma$.

Definição 35. A família de métricas $g_{t}$ é chamada de Deformação de Cheeger de $g$.

Observamos que não há segredo no entendimento da construção supracitada. Em verdade, a ação definida em $M \times G$ coincide com a definição de uma ação em fibrados associados (veja a equação 3.15). Assim, $M$ é recuperada como $M \times_{G} G$. A fim de ilustrar o fenômeno sobre curvar as órbitas de uma ação, considere as seguintes figuras:
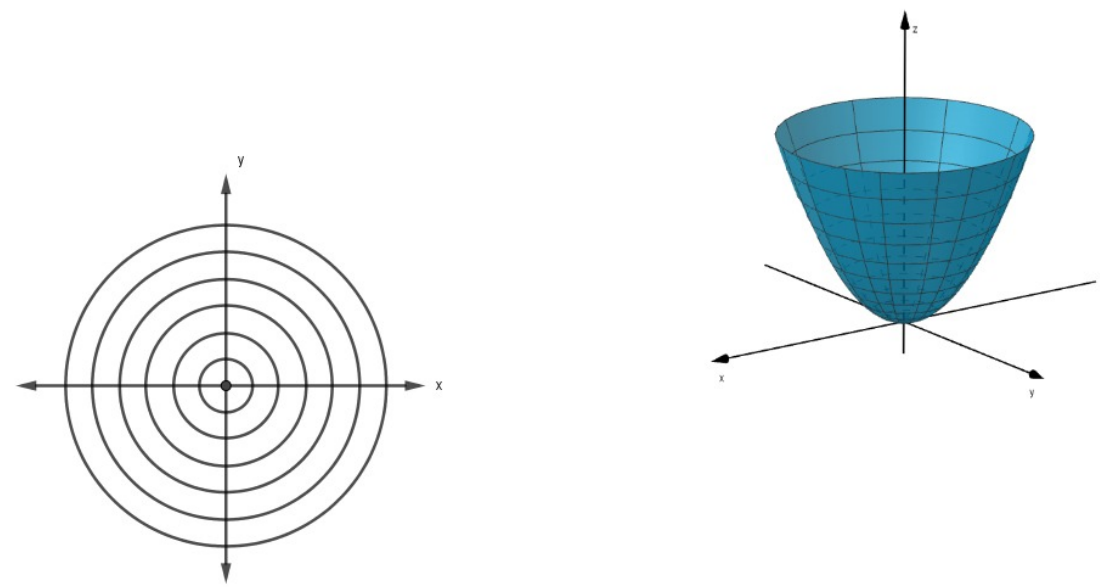

Figura 5.1: A imagem da esquerda representa as órbitas da ação padrão de $U(1)$ em $\mathbb{C}$ e a figura da direita ilustra a deformação de Cheeger da métrica canônica de $\mathbb{C}$ para um tempo $t>0$.

Comecemos por fixar algumas notações. Recorde da seção 3.1 que, dado um ponto $p \in M$, como a ação de $G$ nas órbitas $G \cdot p$ é transitiva, então $G \cdot p$ é difeomorfa ao quociente $G / G_{p}$, onde $G_{p}$ é o grupo de isotropia da ação de $G$ em $p$. Ainda mais, dado que $G$ está munido de uma métrica bi-invariante, vale a decomposição $\mathfrak{g}=\mathfrak{g}_{p} \oplus \mathfrak{m}_{p}$, onde $\mathfrak{g}_{p}$ é a álgebra de Lie do grupo de isotropia $G_{p}$ e $\mathfrak{m}_{p}$ seu complemento ortogonal segundo a métrica $Q$. Recordamos ainda que $\mathfrak{m}_{p}$ é isomorfo ao espaço tangente à órbita em $p, T_{p}(G \cdot p):=\mathcal{V}_{p}$, chamado de espaço vertical. O complemento ortogonal de $\mathcal{V}_{p}$ segundo a métrica $g$ é chamado de espaço horizontal, sendo denotado por $\mathcal{H}_{p}$. Assim, cada vetor tangente $\bar{X} \in T_{p} M$ se escreve de maneira única como $\bar{X}=X+U^{*}$, onde $X$ é um vetor horizontal e $U^{*}=\left.\frac{d}{d t}\right|_{t=0} \exp (t U) \cdot p$, para algum $U \in \mathfrak{m}_{p}$.

A métrica $g_{t}$ se relaciona com a métrica $g$ a partir do tensor $C_{t}$, denominado tensor métrico, via

$$
g_{t}(\bar{X}, \bar{Y})=g\left(C_{t} \bar{X}, Y\right) .
$$

Ademais, pode-se relacionar as métricas $Q$ e $g$ via

$$
g\left(U^{*}, V^{*}\right)=Q(P U, V),
$$


onde $P$ é um operador simétrico e positivo definido. Analogamente

$$
g_{t}\left(U^{*}, V^{*}\right)=Q\left(P_{t} U, V\right) .
$$

Lema 6. As seguintes fórmulas são verdadeiras

$$
C_{t}\left(X+V^{*}\right)=X+\left(P^{-1} P_{t} V\right)^{*}
$$

onde

$$
P_{t}=P(I+t P)^{-1}, I \text { o operador identidade. }
$$

Demonstração. Para verificar a fórmula (5.6) note que o levantamento horizontal de $X+V^{*}$ segundo a submersão $\sigma$ é dado por:

$$
\left(P^{-1}\left(P^{-1}+t I\right)^{-1} V^{*}+X,-t\left(P^{-1}+t I\right)^{-1} V\right) \in T_{p} M \times \mathfrak{g} .
$$

Assim, lembrando que levantamento horizontal é uma isometria, concluímos que:

$$
\begin{aligned}
Q\left(P_{t} U, V\right) & =g_{t}\left(U^{*}, V^{*}\right)=\left(g \times \frac{1}{t} Q\right)\left(\mathcal{L}_{\sigma} U^{*}, \mathcal{L}_{\sigma} V^{*}\right) \\
& =g\left(P^{-1}\left(P^{-1}+t I\right)^{-1} U^{*}, P^{-1}\left(P^{-1}+t I\right) V^{*}\right)+\frac{1}{t} Q\left(-t\left(P^{-1}+t I\right)^{-1} U,-t\left(P^{-1}+t I\right)^{-1} V\right) \\
& \left.=Q\left(\left(P^{-1}+t I\right)^{-1} U, P^{-1}\left(P^{-1}+t I\right)^{-1} V\right)+Q\left(\left(P^{-1}+t I\right)^{-1} U, t\left(P^{-1}+t I\right)^{-1} V\right)\right) \\
& =Q\left(\left(P^{-1}+t I\right)^{-1} U, P^{-1}\left(P^{-1}+t I\right)^{-1} V+t\left(P^{-1}+t I\right)^{-1} V\right) \\
& =Q\left(\left(P^{-1}+t I\right)^{-1} U, V\right) .
\end{aligned}
$$

Por fim, por um lado,

$$
\begin{aligned}
g_{t}\left(U^{*}, V^{*}\right) & =g\left(C_{t} U^{*}, V^{*}\right) \\
& =Q\left(P C_{t} U^{*}, V^{*}\right) .
\end{aligned}
$$

Por outro lado,

$$
g_{t}\left(U^{*}, V^{*}\right)=Q\left(P_{t} U^{*}, V^{*}\right)
$$

Logo, $C_{t}=P^{-1} P_{t}$, de onde obtemos o afirmado.

Como próxima etapa, vamos obter uma fórmula para curvatura seccional da métrica $g_{t}$. Müter em [M̈̈7] fez a observação fundamental de que ao invés de considerar a curvatura seccional de planos $\{\bar{X}, \bar{Y}\}$, é melhor considerar uma reparametrização desses, a saber, $\left\{C_{t}^{-1} \bar{X}, C_{t}^{-1} \bar{Y}\right\}$.

De fato, o levantamento horizontal de $C_{t}^{-1} \bar{X}$ é dado por $(\bar{X},-t P V)$. Dessa maneira, se o plano $\{\bar{X}, \bar{Y}\}$ tem curvatura seccional não negativa na métrica inicial $g$, então o plano reparametrizado tem curvatura seccional não negativa na métrica $g_{t}$.

Teorema 5.1.1. Sejam $\bar{X}=X+U^{*}, \bar{Y}=Y+V^{*}$ vetores tangentes. Então

$$
\kappa_{t}(\bar{X}, \bar{Y}):=R_{g_{t}}\left(C_{t}^{-1} \bar{X}, C_{t}^{-1} \bar{Y}, C_{t}^{-1} \bar{Y}, C_{t}^{-1} \bar{X}\right)=R_{g}(\bar{X}, \bar{Y}, \bar{Y}, \bar{X})+\frac{t^{3}}{4}|[P U, P V]|_{Q}^{2}+z_{t}(\bar{X}, \bar{Y})
$$

onde $z_{t}$ é dado por:

$$
z_{t}(\bar{X}, \bar{Y})=3 t \max _{\substack{Z \in \mathfrak{g} \\|Z|_{Q}=1}} \frac{\left\{d w_{Z}(\bar{X}, \bar{Y})+\frac{t}{2} Q([P U, P V], Z)\right\}^{2}}{t g\left(Z^{*}, Z^{*}\right)+1}
$$


sendo $d w_{Z}$ definido por:

$$
\begin{aligned}
w_{Z}: T M & \rightarrow \mathbb{R} \\
\bar{X} & \mapsto \frac{1}{2} g\left(\bar{X}, Z^{*}\right),
\end{aligned}
$$

onde $Z^{*}$ é o vetor de ação associado a $Z$.

Ainda mais, se $q \in M^{\text {reg }}, X, Y \in \mathcal{H}_{q}$ e $U \in \mathfrak{g}$, então

$$
\begin{aligned}
d w_{Z}\left(U^{*}, X\right) & =\frac{1}{2} X g\left(U^{*}, Z^{*}\right) \\
d w_{Z}(X, Y) & =-\frac{1}{2} g\left([X, Y]^{\mathcal{V}}, Z^{*}\right)=-g\left(A_{X} Y, Z^{*}\right),
\end{aligned}
$$

onde A é o tensor de Gray-O'Neill (veja a Definição 4).

Demonstração. Seguindo a equação (3.9),

$$
\begin{aligned}
R_{g_{t}}\left(C_{t}^{-1} \bar{X}, C_{t}^{-1} \bar{Y}, C_{t}^{-1} \bar{Y}, C_{t}^{-1} \bar{X}\right) & =R_{g \times \frac{1}{t} Q}((\bar{X},-t P U),(\bar{Y},-t P V))+3\left|A_{(\bar{X},-t P U)}^{\sigma}(\bar{Y},-t P V)\right|_{g \times \frac{1}{t} Q}^{2}, \\
& =R_{g}(\bar{X}, \bar{Y})+\frac{1}{t} R_{Q}(t P U, t P V)+3\left|A_{(X,-t P U)}^{\sigma}(Y,-t P V)\right|_{g \times \frac{1}{t} Q}^{2}, \\
& =R_{g}(\bar{X}, \bar{Y})+\frac{t^{3}}{4}|[P U, P V]|_{Q}^{2}+3\left|A_{(\bar{X},-t P U)}^{\sigma}(\bar{Y},-t P V)\right|_{g \times \frac{1}{t} Q}^{2},
\end{aligned}
$$

onde $A^{\sigma}$ denota o tensor de Gray-O'Neill associado à submersão $\sigma$ e usamos que a curvatra seccional da métrica bi-invariante $Q$ é dada por,

$$
R_{Q}(U, V):=\frac{1}{4}|[U, V]|_{Q}^{2}
$$

Por fim, note que as equações (5.11) e (5.12) seguem da definição padrão de derivada exterior de 1-formas:

$$
d \omega(X, Y):=X \omega(Y)-Y \omega(X)-\omega([X, Y]) .
$$

Ademais:

Afirmação 2. Sejam pr $:(M, g) \rightarrow(M / G, \bar{g})$ uma submersão riemanniana e $X, Y$ campos vetoriais horizontais. Então

$$
\left|A_{X} Y\right|_{g}^{2}=\max _{Z \in \mathfrak{g},|Z|=1}\left\{d w_{Z}(X, Y)^{2} g\left(Z^{*}, Z^{*}\right)^{-1}\right\} .
$$

Demonstração. Isso é uma consequência imediata do seguinte fato válido em espaços vetoriais:

Se $V$ é um espaço vetorial com produto interno $\langle\cdot, \cdot\rangle$, então

$$
\langle v, v\rangle=\max _{x \in V-\{0\}}\langle x, v\rangle^{2}\langle x, x\rangle^{-1}
$$

Portanto,

$$
\begin{aligned}
\left|A_{X} Y\right|_{g}^{2} & =\max _{Z \in \mathfrak{g}-\{0\}}\left\{g\left(A_{X} Y, Z^{*}\right)^{2} g\left(Z^{*}, Z^{*}\right)^{-1}\right\}, \\
& =\max _{Z \in \mathfrak{g},|Z|=1}\left\{d w_{Z}(X, Y)^{2} g\left(Z^{*}, Z^{*}\right)^{-1}\right\} .
\end{aligned}
$$

Agora aplicamos a Afirmação 2 à submersão riemanniana $\pi:\left(M \times G, g \times \frac{1}{t} Q\right) \rightarrow\left(M, g_{t}\right)$. Denote por $\bar{w}_{Z}^{t}, w_{Z}$ e $\bar{w}_{Z}$ as 1-formas auxiliares (veja a equação (5.10)) associadas às ações definidas em $\left(M \times G, g \times \frac{1}{t} Q\right),(M, g)$, e à ação de translação à esquerda em $(G, Q)$, respectivamente. Note que $\bar{w}_{Z}^{t}=w_{Z}+t^{-1} \bar{w}_{Z}$. 
Por um lado, $\mathcal{L}_{\sigma} \bar{X}$, o levantamento horizontal de $C_{t}^{-1} \bar{X}$ com respeito à $\sigma: M \times G \rightarrow M$, é dado por

$$
\mathcal{L}_{\sigma} C_{t}^{-1} \bar{X}=(\bar{X},-t P U) .
$$

Notando que $d \bar{w}_{Z}(P U, P V)=\frac{1}{2} Q([P U, P V], Z)$ temos

$$
\begin{aligned}
d \bar{w}_{Z}^{t}\left(\mathcal{L}_{\sigma} C_{t}^{-1} \bar{X}, \mathcal{L}_{\sigma} C_{t}^{-1} \bar{Y}\right) & =d \bar{w}_{Z}^{t}((\bar{X},-t P U),(\bar{Y},-t P V)), \\
& =d w_{Z}(\bar{X}, \bar{Y})+\frac{1}{t} d \bar{w}_{Z}(-t P U,-t P V), \\
& =d w_{Z}(\bar{X}, \bar{Y})+\frac{t}{2} Q([P U, P V], Z) .
\end{aligned}
$$

Para concluir a prova observamos que:

$$
\left(g \times \frac{1}{t} Q\right)\left(Z^{*}, Z^{*}\right)=g\left(Z^{*}, Z^{*}\right)+\frac{1}{t} Q(Z, Z)=g\left(Z^{*}, Z^{*}\right)+\frac{1}{t}=\frac{1}{t}\left(\operatorname{tg}\left(Z^{*}, Z^{*}\right)+1\right)
$$

uma vez que $Q(Z, Z)=1$. A demonstração segue aplicando a Afirmação 2 para métrica $g \times \frac{1}{t} Q$.

Antes de prosseguir e fornecer fórmulas para curvaturas de Ricci e escalar, primeiramente introduzidas em [CS18c, CS18a], apresentamos um teorema original, com uma prova muito simples, a fim de parcialmente convencer o leitor da importância das deformações de Cheeger em curvatura positiva. Esse teorema será generalizado posteriormente na Seção 5.7. A hipótese apresentada é da mesma natureza da do teorema principal em [CDR92], escrito por Chavez-Derdzinski-Rigas (CDR), o que justifica a nomenclatura abaixo:

Teorema 5.1.2 (Pequena Condição CDR). Seja M uma variedade compacta e conexa munida de uma ação isométrica por um grupo de Lie compacto e conexo G. Assuma que as órbitas da ação de $G$ em $M$ sejam positivamente curvadas na métrica de espaço homogêneo normal e que para cada $p \in M$ e cada par de vetores unitários $X, Y+V^{*} \in T_{p} M$ satisfazendo $X \perp Y$, tenhamos que $\kappa_{0}(X, Y+V)>0$. Então $M$ admite uma métrica de curvatura seccional positiva depois de uma deformação de Cheefer finita.

Observação. A hipótese $\kappa_{0}(X, Y+V)=K_{g}(X, Y+V)>0$ é bastante restritiva (veja por exemplo os resultados em [Spe16c]). Por isso, mesmo em posse desse resultado, ainda é particularmente difícil construrir exemplos de variedades com curvatura seccional positiva.

Demonstração. Suponha por contradição que para cada $n \in \mathbb{N}$ existam $p_{n} \in M$ e um par de vetores unitários $\overline{X_{n}}, \overline{Y_{n}}$ tais que $\kappa_{n}\left(\bar{X}_{n}, \bar{Y}_{n}\right) \leq 0$. Pela hipótese de órbitas positivamente curvadas segue que existe $C>0$ tal que $\frac{n^{3}}{4}\left|\left[P U_{n}, P V_{n}\right]\right|_{Q}^{2} \geq \frac{n^{3}}{4} C\left|P U_{n}\right|^{2}\left|P V_{n}\right|^{2} \geq 0$. Assim,

$$
\begin{aligned}
& 0 \geq \kappa_{0}\left(\bar{X}_{n}, \bar{Y}_{n}\right)+\frac{n^{3}}{4}\left|\left[P U_{n}, P V_{n}\right]\right|_{Q}^{2}+z_{n}\left(\bar{X}_{n}, \bar{Y}_{n}\right) \geq \\
& 0 \geq \kappa_{0}\left(\bar{X}_{n}, \bar{Y}_{n}\right)+C \frac{n^{3}}{4}\left|P U_{n}\right|^{2}\left|P V_{n}\right|^{2}+z_{n}\left(\bar{X}_{n}, \bar{Y}_{n}\right) .
\end{aligned}
$$

Dado que $M$ é compacta e $\bar{X}_{n}, \bar{Y}_{n}$ são unitários, passando para subsequências $p_{n_{k}} \rightarrow \tilde{p}$ e $\bar{X}_{n_{k}} \rightarrow$ $\tilde{X}, \bar{Y}_{n_{k}} \rightarrow \tilde{Y}$, onde $\tilde{X}=X+U^{*}, \tilde{Y}=Y+V^{*}$ concluímos que $P U=0$ ou $P V=0$. Sem perda de generalidade, suponhamos que $P U=0$. Então

$$
0 \geq \kappa_{0}(X, Y+V)+\lim _{n \rightarrow \infty} z_{n}(X, Y+V) .
$$

Como $\lim _{n \rightarrow \infty} z_{n}(X, Y+V) \geq 0$ concluímos que

$$
\kappa_{0}(X, Y+V) \leq 0
$$

o que é uma contradição. 
Findada devida digressão, deduziremos expressões para curvaturas de Ricci e escalar. Esse é o objetivo das próximas linhas.

Considere $\bar{X}=X+U^{*} \in T_{p} M$ e uma base ortonormal $\left\{e_{1}, \ldots, e_{n}\right\}$ de $T_{p} M$, onde $\left\{e_{k+1}, \ldots, e_{n}\right\}$ é uma base para $\mathcal{H}_{p}$. Definimos a curvatura horizontal de Ricci por

$$
\operatorname{Ric}^{\mathcal{H}}(\bar{X}):=\sum_{i=k+1}^{n} R\left(\bar{X}, e_{i}, e_{i}, \bar{X}\right)
$$

Lema 7. Para $\bar{X} \in T_{p} M$,

$$
\lim _{t \rightarrow \infty} \operatorname{Ric}_{g_{t}}(\bar{X})=\operatorname{Ric}_{g}^{\mathcal{H}}(X)+\lim _{t \rightarrow \infty} \sum_{i=1}^{n} z_{t}\left(C_{t} \bar{X}, C_{t}^{1 / 2} e_{i}\right)+\frac{1}{4} \sum_{j}\left|\left[v_{j}, U\right]\right|_{Q}^{2},
$$

onde $\left\{v_{1}, \ldots, v_{k}\right\}$ é uma base $Q$-ortonormal para $\mathfrak{m}_{p}$.

Demonstração. Seja $\left\{v_{1}, \ldots, v_{k}\right\}$ uma $Q$-base ortonormal de autovetores de $P$, com autovalores $\lambda_{1} \leq \ldots \leq \lambda_{k}$. Dada uma $g$-base ortonormal $\left\{e_{k+1}, . ., e_{n}\right\}$ de $\mathcal{H}_{p}$, considere a $g$-base ortonormal $\left\{e_{1}, \ldots, e_{k}, e_{k+1}, \ldots, e_{n}\right\}$ de $T_{p} M$, onde $e_{i}=\lambda_{i}^{-1 / 2} v_{i}^{*}$ para $i \leq k$.

Uma conta direta verifica que o conjunto $\left\{C_{t}^{-1 / 2} e_{i}\right\}_{i=1}^{n}$ é uma $g_{t}$-base ortonormal para $T_{p} M$. Mais ainda, $C_{t}^{-1 / 2} e_{i}=\left(1+t \lambda_{i}\right)^{1 / 2} e_{i}$ para $i \leq k$ e $C_{t}^{-1 / 2} e_{i}=e_{i}$ para $i>k$. Afirmamos que a curvatura de Ricci de $g_{t}$ satisfaz:

$$
\begin{aligned}
\operatorname{Ric}_{g_{t}}(\bar{X})= & \operatorname{Ric}_{g}^{\mathcal{H}}\left(C_{t} \bar{X}\right)+\sum_{i=1}^{n} z_{t}\left(C_{t}^{1 / 2} e_{i}, C_{t} \bar{X}\right) \\
& +\sum_{i=1}^{k} \frac{1}{1+t \lambda_{i}}\left(\kappa_{0}\left(e_{i}, C_{t} \bar{X}\right)+\frac{\lambda_{i} t}{4}\left|\left[v_{i}, t P(1+t P)^{-1} U\right]\right|_{Q}^{2}\right)
\end{aligned}
$$

Para provar a equação (5.14), note que pela equação (5.7) temos

$$
\begin{aligned}
& \operatorname{Ric}_{g_{t}}\left(C_{t}^{-1} \bar{X}\right)=\sum_{i=1}^{n} R_{g_{t}}\left(C_{t}^{-1 / 2} e_{i}, C_{t}^{-1} \bar{X}, C_{t}^{-1} \bar{X}, C_{t}^{-1 / 2} e_{i}\right)=\sum_{i=1}^{n} \kappa_{t}\left(C_{t}^{1 / 2} e_{i}, \bar{X}\right) \\
&=\sum_{i=1}^{n} \kappa_{0}\left(C_{t}^{1 / 2} e_{i}, \bar{X}\right)+\sum_{i=1}^{n} z_{t}\left(C_{t}^{1 / 2} e_{i}, \bar{X}\right)+\frac{t^{3}}{4} \sum_{i=1}^{k}\left|\left[P C_{t}^{1 / 2} \lambda_{i}^{-1 / 2} v_{i}, P \bar{X}_{\mathfrak{g}}\right]\right|_{Q}^{2} \\
&=\operatorname{Ric}_{g}^{\mathcal{H}}(\bar{X})+\sum_{i=1}^{n} z_{t}\left(C_{t}^{1 / 2} e_{i}, \bar{X}\right)+\sum_{i=1}^{k} \frac{1}{1+t \lambda_{i}}\left(\kappa_{0}\left(e_{i}, \bar{X}\right)+\frac{\lambda_{i} t}{4}\left|\left[v_{i}, t P \bar{X}_{\mathfrak{g}}\right]\right|_{Q}^{2}\right) .
\end{aligned}
$$

A equação (5.14) é obtida ao trocar-se $\bar{X}$ por $C_{t} \bar{X}$. Portanto, $\operatorname{Ric}_{g}^{\mathcal{H}}\left(C_{t} \bar{X}\right) \rightarrow \operatorname{Ric}_{g}^{\mathcal{H}}(X)$,

$$
\sum_{i=1}^{k} \frac{1}{1+t \lambda_{i}} \kappa_{0}\left(e_{i}, C_{t} \bar{X}\right) \rightarrow 0
$$

e

$$
\sum_{i=1}^{k} \frac{t \lambda_{i}}{1+t \lambda_{i}} \frac{1}{4}\left|\left[v_{i}, t P(1+t P)^{-1} U\right]\right|_{Q}^{2} \rightarrow \sum_{i=1}^{k} \frac{1}{4}\left|\left[v_{i}, U\right]\right|_{Q}^{2}
$$


A expressão para curvatura escalar é obtida de maneira semelhante, sendo dada por:

$$
\begin{aligned}
\operatorname{scal}_{g_{t}}(p)=\sum_{i, j=1}^{n} K_{g}\left(C_{t}^{1 / 2} e_{i}, C_{t}^{1 / 2} e_{j}\right)+z_{t}\left(C_{t}^{1 / 2} e_{i}, C_{t}^{1 / 2} e_{j}\right) & \\
& +\sum_{i, j=1}^{k} \frac{\lambda_{i} \lambda_{j} t^{3}}{\left(1+t \lambda_{i}\right)\left(1+t \lambda_{j}\right)} \frac{1}{4}\left|\left[v_{i}, v_{j}\right]\right|_{Q}^{2} .
\end{aligned}
$$

\subsection{Sobre a efetividade das deformações de Cheeger clássicas}

Teorema 5.2.1 (Searle-Wilhlem). Seja $(M, g)$ uma $G$-variedade riemanniana onde $M$ e $G$ são compactos. Assuma que

1. Uma órbita principal de $G$ em $M$ possui grupo fundamental finito,

2. $\operatorname{Ric}_{M / G} \geq 1$ na métrica de distância orbital.

Então $M$ admite uma métrica $G$-invariante com curvatura de Ricci positiva.

Como apontamos na Introdução, o Teorema 5.2.1 (apresentado em [SW15]) é fundamental na construção de diversos exemplos de variedades com curvatura de Ricci positiva. Entretanto, a demonstração originalmente apresentada é muito elaborada e baseada em diversas estimativas difíceis. A demonstração consiste em: realizar uma transformação conforme da métrica $g$ seguida de uma deformação de Cheeger. Essa seção é portanto motivada pelas seguintes perguntas:

É possivel provar o Teorema 5.2.1 utilizando apenas deformações de Cheeger? Isso simplificaria a demonstração?

No que segue responderemos completamente essas perguntas, fornecendo condições necessárias e suficientes para que as deformações de Cheeger sejam efetivas como técnica única na prova do Teorema 5.2.1. Mostraremos que tais condições estão diretamente amarradas à ação $G$ (veja o Teorema 5.2.2). Em particular, apresentamos os primeiros exemplos na literatura de variedades satisfazendo as hipóteses do Teorema 5.2.1 que não desenvolvem curvatura de Ricci positiva somente com deformações de Cheeger (veja o Teorema 5.2.4). Por fim, toda a teoria desenvolvida permitiu apresentar uma prova muito mais simples para esse resultado (veja o Teorema 5.4.1)

A seguir listamos alguns resultados a serem provados nesta seção.

Teorema 5.2.2 (Obstrução para efetividade). Seja $\left(M^{n}, g\right)$ uma variedade riemanniana compacta e conexa munida de uma ação isométrica efetiva por um grupo de Lie compacto e conexo G. Denote por $\mathcal{H}_{p}$ o espaço horizontal complementar à órbita da G-ação em p. Assuma que:

1. Uma órbita principal de $G$ em $M$ tenha grupo fundamental finito,

2. $\operatorname{Ric}_{M^{r e g} / G} \geq 1$

Se $g$ tem direções de curvatura de Ricci negativa para cada deformação de Cheeger finita então

(a) Existem um ponto singular $p \in M$ e um vetor não nulo $X \in \mathcal{H}_{p}$ fixado pela representação de isotropia $\rho: G_{p} \rightarrow O\left(\mathcal{H}_{p}\right)$

(b) A restrição de $\rho$ a $X^{\perp} \cap \mathcal{H}_{p}$ é redutivel.

Ainda mais, se $X^{\perp} \cap \mathcal{H}_{p}$ tem exatamente dois fatores $\rho$-iredutiveis, $X^{\perp} \cap \mathcal{H}_{p}=\mathcal{H}_{1}+\mathcal{H}_{2}$, então (a menos de alterar a ordem dos fatores), para qualquer vetor regular $Y=Y_{1}+Y_{2} \in \mathcal{H}_{1}+\mathcal{H}_{2}$,

$$
\operatorname{dim} \mathcal{H}_{1}-\operatorname{dim} G_{p} Y_{1}>\frac{(k-1) \operatorname{dim} \mathcal{H}_{1}}{\operatorname{dim} \mathcal{H}_{p}-1},
$$

onde $k$ é a codimensão de uma órbita principal em $M$. 
Nesse caso, as deformações de Cheeger são ditas inefetivas e existe uma geodésica em $M$ totalmente contida no estrato singular.

Como consequência direta do Teorema 5.2.2 provamos:

Corolário 5.2.3 (Condições suficientes para efetividade). Seja $(M, g)$ uma variedade riemanniana compacta e conexa munida de uma métrica $G$-invariante onde $G$ é um grupo de Lie compacto e conexo. Assuma que:

1. Uma órbita principal $G$ em $M$ tenha grupo fundamental finito,

2. $\operatorname{Ric}_{M^{r e g} / G} \geq 1$,

Então g desenvolve uma métrica de curvatura de Ricci positiva após deformação de Cheeger finita se uma das seguintes (equivalentes) condições é satisfeita:

(a) O estrato singular é composto de órbitas isoladas;

(b) Para todo ponto singular p, a representação linear de isotropia é irredutivel;

(c) Nenhuma representação de isotropia tem um vetor não nulo fixo;

(d) A ação induzida de $G$ no espaço tangente unitário $T_{1} M$ não tem pontos fixos.

Teorema 5.2.4 (Uma família de contra-exemplos). Seja $S^{n}$ a esfera unitária em $\mathbb{R}^{n+1}$ munida da ação padrão de $S O(n-2)$ fixando as 3 primeiras coordenadas. Então, para cada $n \geq 5$ existe uma métrica $S O(n-2)$-invariante $g$ em $S^{n}$ satisfazendo:

1. $\operatorname{Ric}_{M^{\text {reg }} / G} \geq 1$

2. Existe um vetor não nulo $X \in \mathcal{H}_{p}$ tal que $\operatorname{Ric}_{g_{t}}(X)<0$ para toda deformação de Cheeger $g_{t}$.

\subsubsection{A geometria em pontos singulares}

Seja $(M, g)$ uma $G$-variedade riemanniana e denote por $g_{t}$ uma deformação de Cheeger de $g$. Recorde a expressão para curvatura de Ricci de $g_{t}$ :

$\operatorname{Ric}_{g_{t}}(\bar{X})=\operatorname{Ric}_{g}^{\mathcal{H}}\left(C_{t} \bar{X}\right)+\sum_{i=1}^{n} z_{t}\left(C_{t}^{1 / 2} e_{i}, C_{t} \bar{X}\right)+\sum_{i=1}^{k} \frac{1}{1+t \lambda_{i}}\left(\kappa_{0}\left(e_{i}, C_{t} \bar{X}\right)+\frac{\lambda_{i} t}{4}\left|\left[v_{i}, t P(1+t P)^{-1} U\right]\right|_{Q}^{2}\right)$.

A maior dificuldade em garantir que $g_{t}$ desenvolva curvatura de Ricci positiva reside nos pontos singulares. Lembrando o Teorema 3.1.9, existe um aberto denso e convexo $M^{\text {reg }}$ em $M$ com a porpriedade de que dados dois pontos $p, q \in M^{r e g}$, os subgrupos de isotropia $G_{p}$ e $G_{q}$ são conjugados.

Em pontos singulares o tensor $P$ perde posto, e a dimensão do espaço horizontal aumenta. Essa perda de posto afeta diretamente o termo que provém de $\frac{t^{3}}{4}|[P U, P V]|_{Q}^{2}$, o que somado ao acréscimo de direções na curvatura horizontal de Ricci, faz com que uma garantia para efetividade das deformações de Cheeger se dê pelo estudo do tensor $z_{t}$ (veja o Teorema 5.1.1).

Dessa maneira, o objetivo desta seção consiste em entender o comportamento do tensor $z_{t}$ em pontos sigulares, i.e, $p \in M \backslash M^{r e g}$. Faremos isso considerando geodésicas partindo de tais

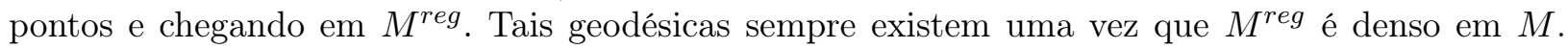
Mostraremos que sob certas condições, $z_{t}$ apresenta compartamento de blow-up, o que garante condições suficientes para existência de curvatura de Ricci positiva via deformações de Cheeger.

Precisamente, seja $\mathcal{O} \subset M$ uma órbita singular em $M$ e seja $\gamma(s)$ uma geodésica horizontal começando em um ponto $p \in \mathcal{O}$ com direção $X \in \mathcal{H}_{p}$. Assuma que $\forall s>0$ pequeno tenhamos que $\gamma(s) \subset M^{r e g}$. Naturalmente o transporte dos espaços horizontais e verticais ao longo de $\gamma$ não preserva dimensão, uma vez que a isotropia em $p$ é maior que na parte regular. Alguns subespaços de $\mathcal{H}_{p}$ podem ser obtidos como limite do transporte paralelo de subespaços de $T_{\gamma(s)} M$ ao longo $\gamma(s), s \rightarrow 0^{+}$. Se existir um vetor $Y \in \mathcal{H}_{p}$ que é limite (ao longo de $\gamma(s)$ ) de um vetor vertical, ou, ao 
menos tenha uma compente obtida dessa maneira, então o tensor $z_{t}(X, Y)$ apresenta comportamente de blow-up (no parâmetro $t$ ) (esse é o conteúdo da Proposição 5.2.6). Começaremos por descrever como parametrizar o subespaço de tais vetores $Y$.

Fixado $p \in \mathcal{O}$, como a ação se dá por isometrias, o espaço $\mathcal{H}_{p}$ é invariante pela restrição de $\rho$ ao subgrupo de isotropia de $p$. Assim, podemos considerar $\rho: G_{p} \rightarrow O\left(\mathcal{H}_{p}\right)$, a restrição da ação de $G$ ao subgrupo de isotropia em $p$. Considerando a diferencial $d \rho: \mathfrak{g}_{p} \rightarrow \mathfrak{o}\left(\mathcal{H}_{p}\right)$, podemos ver $d \rho(U)$ como um operador antissimétrico em $\mathcal{H}_{p}$, dado que $U^{*}$ é um campo de Killing ao longo de geodésicas horizontais. Mais precisamente, como o mapa exponencial define uma aplicação $G_{p}$-equivariante entre $T_{p} M$ e $M$, vale que

$$
d \rho(U) X=\left(\nabla_{X} U^{*}\right)_{p} .
$$

Seja $X \in \mathcal{H}_{p}$ de tal maneira que a geodésica $\gamma(s):=\exp _{p}(s X)$ encontre o estrato regular para todo $s>0$. Assim, para cada vetor de ação $U^{*}$, denote por $U^{*}(s):=U^{*}(\gamma(s))$ a extensão de $U^{*}$ ao longo de $\gamma$ e lembre-se que $U^{*}(s)$ é um campo de Jacobi. Defina:

$$
\begin{gathered}
\tilde{S}_{X}: \mathfrak{g} \rightarrow T_{p} M, \\
U \mapsto\left(\nabla_{X} U^{*}\right)(0) .
\end{gathered}
$$

O próxima lema caracteriza completamente os subespaços $\mathcal{W} \subset \mathcal{H}_{p}$ tais que $P_{\gamma(s)}(\mathcal{W}) \subset$ $\mathcal{H}_{\gamma(s)}, s>0$, onde $P_{\gamma(s)}(\mathcal{W})$ denota o transporte paralelo de $\mathcal{W}$ ao longo de $\gamma(s)$ no instante $s$.

Lema 8. Sejam $p$ um ponto singular e $X \in \mathcal{H}_{p}$. Considere $\gamma(s):=\exp _{p}(s X)$ e assuma que $\forall s>$ $0, \exp _{p}(s X) \subset M^{r e g}$. Então,

1. $\tilde{S}_{X}\left(\mathfrak{g}_{p}\right) \subset \mathcal{H}_{p}$. Mais ainda, para $\epsilon>0$ pequeno o bastante,

$$
\tilde{\mathcal{H}}_{t}:=\left\{\begin{array}{l}
\mathcal{H}_{\gamma(s)}, s \neq 0 \\
\left(\tilde{S}_{X}\left(\mathfrak{g}_{p}\right)\right)^{\perp} \cap \mathcal{H}_{p}, s=0
\end{array}\right.
$$

é um fibrado suave ao longo de $\gamma(-\epsilon, \epsilon)$.

2. $\mathfrak{g}_{p} \cap \operatorname{ker} \tilde{S}_{X}=\mathfrak{g}_{X}$, onde $\mathfrak{g}_{X}$ é a álgebra de Lie de $G_{X}:=\{g \in M: \rho(g) X=X\}$.

Em particular, o subespaço $\mathcal{W} \subset \mathcal{H}_{p}$ que provém de vetores horizontais ao longo de $\gamma(s)$ é dado pelo complemento ortogonal da imagem de $\tilde{S}_{X}\left(\mathfrak{g}_{p}\right)$.

Demonstração. Seguindo [GW09, Section 1.7, p. 35], definimos

$$
\mathcal{J}:=\operatorname{span}\left\{\left.U^{*}\right|_{\gamma}: U \in \mathfrak{g}\right\} \cup\left\{J: J(0)=0, J^{\prime}(0) \in \mathcal{H}_{p}\right\}
$$

A família $\mathcal{J}$ satisfaz:

$$
g\left(J_{1}^{\prime}(s), J_{2}(s)\right)=g\left(J_{1}(s), J_{2}^{\prime}(s)\right), \forall J_{1}, J_{2} \in \mathcal{J},
$$

ou seja, $\mathcal{J}$ é um família de campos $(n-1)$-dimensional de Jacobi ortogonais a $\gamma$ com operador de Ricatti auto-adjunto. Dessa maneira, segue que:

$$
\dot{\gamma}(s)^{\perp}=\operatorname{span}\{J(s): J \in \mathcal{J}\} \stackrel{\perp}{\oplus}\left\{J^{\prime}(s) \in \mathcal{J}: J(s)=0\right\} \subset T_{\gamma(s)} M .
$$

Se $U^{*}(0)=0$, de acordo com a equação (5.19) temos que para todo $V \in \mathfrak{g}$,

$$
g\left(\nabla_{X} U^{*}(0), V^{*}(0)\right)=g\left(U^{*}(0), \nabla_{X} V^{*}(0)\right)=0,
$$

de onde segue que $\nabla_{X} U^{*}(0) \in \mathcal{H}_{p}$. A segunda afirmação no primeito item segue da discussão em [GW09]. 
Por fim, uma vez que

$$
\mathfrak{g}_{X}=\{V \in \mathfrak{g}: d \rho(V) X=0\}
$$

e que para qualquer $U \in \mathfrak{g}_{p}$

$$
\tilde{S}_{X}(U)=d \rho(U) X
$$

temos

$$
U \in \mathfrak{g}_{p} \cap \operatorname{ker} \tilde{S}_{X} \Leftrightarrow d \rho(U) X=0 \Leftrightarrow U \in \mathfrak{g}_{X}
$$

Denote por

$$
\mathfrak{p}_{X}:=\mathfrak{g}_{p} \ominus \mathfrak{g}_{X} \equiv \mathfrak{g}_{p} \cap\left(\mathfrak{g}_{X}\right)^{\perp}
$$

Então vale que:

Corolário 5.2.5. O mapa $\left.\tilde{S}_{X}\right|_{\mathfrak{p}_{X}}: \mathfrak{p}_{X} \rightarrow \mathcal{H}_{p}$ é injetor.

Definição 36. Seja $p \in M$ um ponto singular e $X \in \mathcal{H}_{p}$. Elementos na imagem $\tilde{S}_{X}\left(\mathfrak{p}_{X}\right)$ são chamados de falso horizontais com respeito a $X$. Dado $Y \in \mathcal{H}_{p}$, denotamos por $Y_{\mathfrak{p}_{X}}$ o único elemento de $\mathfrak{p}_{X}=\mathfrak{g}_{p} \ominus \mathfrak{g}_{X}$ tal que $\tilde{S}_{X} Y_{\mathfrak{p}_{X}}$ é a projeçãa ortogonal de $Y$ em $\tilde{S}_{X}\left(\mathfrak{g}_{p}\right)$.

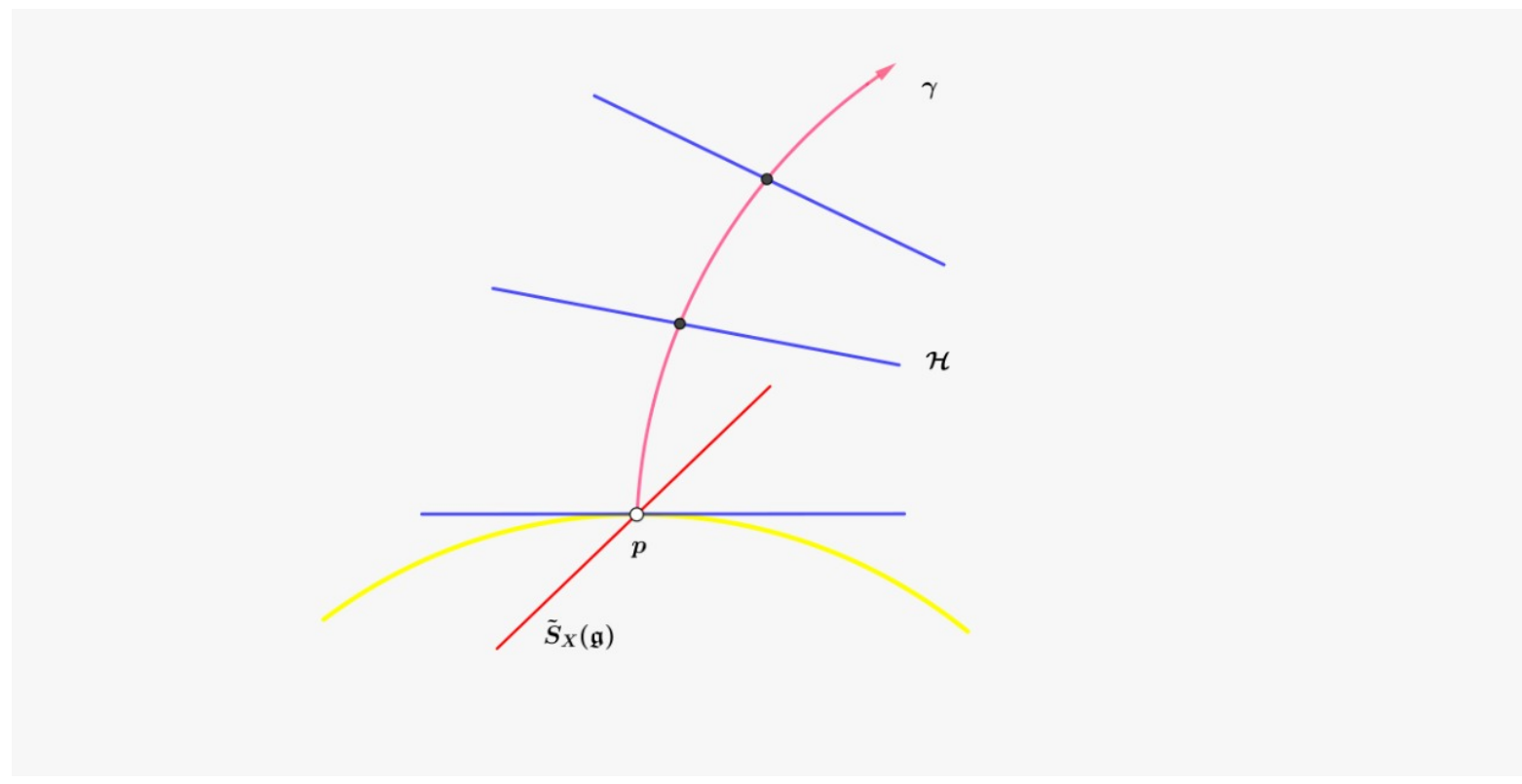

Figura 5.2: Na imagem o ponto p representa um ponto numa órbita singular (pintada de amarelo). As linhas em azul representam as fibras do fibrado horizontal na parte regular e a reta em verlho o subespaço dos vetores falsos horizontais.

A próxima proposição mostra que o tensor $z_{t}$ computado num plano $X, Y \in \mathcal{H}_{p}$, onde $p$ é um ponto singular, apresenta comportamento de blow-up (no parâmetro $t$ da deformação de Cheeger) discutido acima.

Para as seguintes demonstrações assumiremos que a geodésica $\gamma(s)$ partindo de um ponto singular $p$ na direção de um vetor horizontal $X \in \mathcal{H}_{p}$ intercepta uma órbita principal para algum $s$ e, portanto, num conjunto denso e aberto do domínio de $\gamma$. Assim, por simplicidade, sempre diremos que $\gamma(s) \subset M^{r e g}, \forall s>0$. Embora isso não seja necessariamente verdade para cada escolha do vetor horizontal $X$, há um conjunto aberto e denso de direções com essa propriedade. Pela continuidade de $z_{t}$ as estimativas valem para todas as direções, e portanto, vamos nos restringir a esse caso, sem perda de generalidade. 
Proposição 5.2.6. Seja $p$ um ponto singular em $M$ e $X, Y \in \mathcal{H}_{p}$. Então

$$
z_{t}(X, Y) \geq 3 t \frac{\left|\tilde{S}_{X}\left(Y_{\mathfrak{p}_{X}}\right)\right|_{g}^{4}}{\left|Y_{\mathfrak{p}_{X}}\right|_{Q}^{2}}
$$

Demonstração. Sejam $p \in \mathcal{O} \subset M$ um ponto singular e $X \in \mathcal{H}_{p}$. Considere a geodésica horizontal $\gamma(s):=\exp _{p}(s X)$ e assuma que $\forall s>0, \gamma(s) \subset M^{r e g}$. Seja $U \in \tilde{S}_{X}\left(\mathfrak{g}_{p}\right)$ e tome

$$
U(s):=\frac{1}{s} U_{\mathfrak{p}_{X}}^{*}(s) .
$$

Note que $U(0)=U$. De fato,

$$
\lim _{s \rightarrow 0^{+}} U(s)=\lim _{s \rightarrow 0^{+}} \frac{1}{s} U_{\mathfrak{p}_{X}}^{*}(s)=\nabla_{X} U_{\mathfrak{p}_{X}}^{*}(0)=d \rho\left(U_{\mathfrak{p}_{X}}\right) X=U
$$

Vamos computar $d w_{Z}(U, X)$. Para tanto, para qualquer $W \in \mathfrak{g}$ dado, denotaremos $W^{*}(s):=$ $W^{*}(\gamma(s))$.

Afirmação 3. Dado $U \in \tilde{S}_{X}\left(\mathfrak{p}_{X}\right), \lim _{s \rightarrow 0^{+}} d w_{Z}(U(s), X(s))=g\left(U, \nabla_{X} Z^{*}(0)\right)$.

Demonstração. Primeiro notamos que a equação (5.11) garante que como $U_{\mathfrak{p}_{X}} \in \mathfrak{g}_{p}$ (e portanto, $\left.U_{\mathfrak{p}_{X}}^{*}(0)=0\right)$, então

$$
\left.\frac{1}{2} X g\left(U_{\mathfrak{p}_{X}}^{*}, Z^{*}\right)\right|_{s=0}=d w_{Z}\left(U_{\mathfrak{p}_{X}}^{*}(0), X\right)=0 .
$$

Para $s>0$, pela equação (5.11) tem-se

$$
d w_{Z}(U(s), X(s))=\frac{1}{2 s} X g\left(U_{\mathfrak{p}_{X}}^{*}(s), Z^{*}(s)\right) .
$$

Portanto, pela equação (5.22)

$$
\begin{aligned}
& \lim _{s \rightarrow 0^{+}} d w_{Z}(U(s), X(s))=\left.\frac{1}{2} \frac{\partial^{2}}{\partial s^{2}}\right|_{s=0} g\left(U_{\mathfrak{p}_{X}}^{*}(s), Z^{*}(s)\right) \\
& =\frac{1}{2}\left\{g\left(\frac{D^{2}}{d s^{2}} U_{\mathfrak{p}_{X}}^{*}(0), Z^{*}(0)\right)+g\left(U_{\mathfrak{p}_{X}}^{*}(0), \frac{D^{2}}{d s^{2}} Z^{*}(0)\right)+2 g\left(\frac{D}{d s} U_{\mathfrak{p}_{X}}^{*}(0), \frac{D}{d s} Z^{*}(0)\right)\right\} .
\end{aligned}
$$

A afirmação segue portanto do fato que $\frac{D^{2}}{d s^{2}} U_{\mathfrak{p}_{X}}^{*}(0)=-R\left(U_{\mathfrak{p}_{X}}^{*}(0), X(0)\right) X(0)=0$ e $\frac{D}{d s} U_{\mathfrak{p}_{X}}^{*}(0)=$ $U$.

Para concluir a prova da Proposição note que, como $z_{t}(X, Y) \geq 0$, o resultado segue imediatamente para $Y \perp \tilde{S}_{X}\left(\mathfrak{g}_{p}\right)$ (porque neste caso, $Y_{\mathfrak{p}_{X}}=0$ ). Do contrário, pegamos $Z=Y_{\mathfrak{p}_{X}} /\left|Y_{\mathfrak{p}_{X}}\right|_{Q}$ na equação (5.8) e usamos a Afirmação 3 , de onde

$$
z_{t}(X, Y) \geq 3 t \frac{g\left(Y, \frac{D}{d s} Z^{*}(0)\right)^{2}}{\operatorname{tg}\left(Z^{*}(0), Z^{*}(0)\right)+1}=3 t g\left(Y, \frac{\tilde{S}_{X} Y_{\mathfrak{p}}}{\left|Y_{\mathfrak{p}_{X}}\right|_{Q}}\right)^{2}=3 t \frac{\left|\tilde{S}_{X} Y_{\mathfrak{p}_{X}}\right|_{g}^{4}}{\left|Y_{\mathfrak{p}_{X}}\right|_{Q}^{2}}
$$

já que $Z^{*}(0)=0$ e $\frac{D}{d s} Z^{*}(0)=\frac{\tilde{S}_{X} Y_{\mathfrak{p}}}{\left|Y_{\mathfrak{p}}\right|_{Q}}$.

Estamos em condições de provar o item (a) do Teorema 5.2.2. Comecemos por provar o seguinte:

Afirmação 4. Considere a submersão riemanniana $\pi:\left(M^{r e g}, g\right) \rightarrow\left(M^{r e g} / G, \bar{g}\right)$. Se $p \in M^{r e g}$ então

$$
\lim _{t \rightarrow \infty} \operatorname{Ric}_{g_{t}}(X)=\operatorname{Ric}_{\bar{g}}(d \pi X)
$$


Demonstração. A Afirmação 4 segue da Prposição 3.3 de [SW15], nós forneceremos uma prova por questão de conveniência. Observe que:

$$
\lim _{t \rightarrow \infty} \operatorname{Ric}_{g_{t}}(X)=\operatorname{Ric}_{g}^{\mathcal{H}}(X)+\sum_{i=1}^{n} \lim _{t \rightarrow \infty} z\left(C_{t}^{1 / 2} e_{i}, X\right),
$$

desde que $\lim z_{t}\left(C_{t}^{1 / 2} e_{i}, X\right)$ exista para todo $i$.

O Teorema 5.1.1 diz que

$$
\begin{array}{r}
z_{t}(X, Y)=3 \max _{\substack{Z \in \mathfrak{g} \\
|Z|_{Q}=1}}\left\{\frac{g\left(A_{X} Y, Z^{*}\right)^{2}}{g\left(Z^{*}, Z^{*}\right)+t^{-1}}\right\} \\
z_{t}\left(X, W^{*}\right)=3 \max _{\substack{Z \in \mathfrak{g} \\
|Z|_{Q}=1}}\left\{\frac{g\left(S_{X} W^{*}, Z^{*}\right)^{2}}{g\left(Z^{*}, Z^{*}\right)+t^{-1}}\right\}
\end{array}
$$

para todos $Y \in \mathcal{H}_{p}, W \in \mathfrak{g}$. Em particular, $z_{t}\left(C_{t}^{1 / 2} e_{i}, X\right) \rightarrow 0$ para $i \leq k$, já que $C_{t}^{1 / 2} e_{i} \rightarrow 0$. Por outro lado, a fórmula de Gray-O’Neill (veja (3.3)) para submersão riemanniana diz que,

$$
K_{\bar{g}}(d \pi X, d \pi Y)-K_{g_{t}}(X, Y)=3\left|A_{X} Y\right|_{g}^{2}-z_{t}(X, Y) .
$$

Assim, a Afirmação segue do fato que $z_{t}(X, Y) \rightarrow 3\left|A_{X} Y\right|_{g}^{2}$.

Demonstração do Teorema 5.2.2 item (a). Assuma que para cada $n \in \mathbb{N}$ exista $\bar{X}_{n}$ unitário tal que

$$
\operatorname{Ric}_{g_{n}}\left(\bar{X}_{n}\right) \leq 0 .
$$

Passando a uma subsequência, se necessário, obtemos um vetor unitário $\bar{X}=X+U^{*}$ num ponto limite $p$ tal que:

$$
\lim _{n \rightarrow \infty} \operatorname{Ric}_{g_{n}}\left(\bar{X}_{n}\right)=\operatorname{Ric}^{\mathcal{H}}(X)+\lim _{n \rightarrow \infty} \sum_{i=k+1}^{n} z_{t}\left(X, e_{i}\right)+\frac{1}{4} \sum_{j}\left|\left[v_{j}, U\right]\right|_{Q}^{2} \leq 0 .
$$

Essa expressão permite concluir que $X \neq 0$. De fato, como as órbitas de $G$ em $M$ tem grupo fundamental finito, existe $C>0$ tal que $\sum_{j}\left|\left[v_{j}, U\right]\right|^{2} \geq C|U|^{2}$ e, portanto, a soma em $j$ é positiva desde que $U \neq 0$. Daí, se $X=0$, teríamos uma contradição pois $z_{t}$ também é não negativo. Por outro lado, $p$ é um ponto singular, pois do contrário, pela Afirmação 4 teríamos $\lim _{t \rightarrow \infty} \operatorname{Ric}_{g_{t}}(X)=$ $\operatorname{Ric}(d \bar{\pi} X)$ e por hipótese o quociente tem curvatura de Ricci positiva.

Ademais, $\mathfrak{g}_{p}=\mathfrak{g}_{X}$ pois do contrário, $\mathfrak{p}_{X} \neq \emptyset$ e como $p$ é singular, $\left.\tilde{S}_{X}\right|_{\mathfrak{p}_{X}}$ define uma injeção em $\mathcal{H}_{p}$, então pela Proposição 5.2.6, teríamos $\lim _{t \rightarrow \infty} z_{t}\left(X, e_{i}\right)=\infty$, para algum $i$.

Isso conclui a demonstração.

\subsubsection{Eixos fixos e uma descrição algébrica da curvatura de Ricci}

Como vimos na demonstração do Teorema 5.2.2, item (a), a (não) efetividade da deformação está diretamente ligada à existência de eixos fixos, isto é, vetores horizontais não nulos $X$ tais que $\mathfrak{g}_{X}=\mathfrak{g}_{p}$. Nesta subseção apresentaremos uma discussão algébrica do tensor de Ricci nas direções de tais vetores, que será usada posteriormente para fornecer os primeiros exemplos de variedades satisfazendo as hipóteses do Teorema de Searle-Wilhelm (Teorema 5.2.1) e que não desenvolvem curvatura de Ricci positiva somente com deformações de Cheeger.

De agora em diante, consideramos uma variedade riemanniana $(M, g)$ compacta, conexa e equipada com uma ação isométrica (efetiva) por um grupo de Lie compacto e conexo $G$, satisfazendo a hipótese em Teorema 5.2.1. A próxima proposição garante que a não efetividade da deformação de Cheeger está relacionada à curvatura horizontal de Ricci em eixos fixos. 
Definição 37. Seja $p \in M$ um ponto singular e $X \neq 0 \in \mathcal{H}_{p}$ um vetor tal que $\mathfrak{g}_{p}=\mathfrak{g}_{X}$. Nesse caso diremos que $X$ é um eixo fixo.

Proposição 5.2.7. Sejam $p$ um ponto singular e $X \in \mathcal{H}_{p}$ um eixo fixo. Então, para todo $Y \in \mathcal{H}_{p}$, vale que

$$
z_{t}(X, Y)=0, \forall t .
$$

Em particular, $\lim _{t \rightarrow \infty} \operatorname{Ric}_{g_{t}}(X)=\operatorname{Ric}_{g}^{\mathcal{H}}(X)$.

Demonstração. Pela fórmula (5.14) é suficiente provar que $d w_{Z}(X, Y)=0, \forall Z \in \mathfrak{g}$.

Pela definição de derivada exterior, levando em conta que $Z^{*}$ é um campo de Killing, temos:

$$
\begin{aligned}
2 d w_{Z}(X, Y) & =X g\left(Z^{*}, Y\right)-Y g\left(Z^{*}, X\right)-g\left([X, Y], Z^{*}\right) \\
& =g\left(\nabla_{X} Z^{*}, Y\right)+g\left(Z^{*}, \nabla_{X} Y\right)-g\left(\nabla_{Y} Z^{*}, X\right)-g\left(Z^{*}, \nabla_{Y} Z\right)-g\left([X, Y], Z^{*}\right) \\
& =-2 g\left(\nabla_{Y} Z^{*}, X\right)+g\left(Z^{*},[X, Y]\right)-g\left([X, Y], Z^{*}\right) \\
& =2 g\left(\nabla_{X} Z^{*}, Y\right) \\
& =0
\end{aligned}
$$

onde a última igualdade segue do fato que $\tilde{S}_{X}=0$.

A subseção 5.2.1 e a Proposição 5.2.7 permitem concluir que variedades satisfazendo as hipótese do Teorema 5.2.1 tem curvatura de Ricci positiva para algum $t$ se, e somente se, $\operatorname{Ric}_{g}^{\mathcal{H}}(X)>0$ para todo eixo fixo $X$. Isso vai ser essencial para uma demonstração mais simples do Teorema 5.2.1. Registramos isso como um corolário:

Corolário 5.2.8. Suponha que $(M, g)$ satisfaça

1. Uma órbita principal tem grupo fundamental finito

2. $\operatorname{Ric}_{M^{r e g} / G} \geq 1$

Então existe $t>0$ tal que $g_{t}$ possui curvatura de Ricci positiva se, e somente se, $\operatorname{Ric}_{g}^{\mathcal{H}}(X)>0$ para todo eixo fixo $X_{p} \in \mathcal{H}_{p}, \forall p \in M$.

\section{O tensor de Ricci nos eixos fixos}

Nas próximas linhas entederemos o comportamento da curvatura horizontal de Ricci obtida como limite de subespaços de espaços horizontias na parte regular, o que permite concluir a prova do item (b) do Teorema 5.2.2. Mostramos também como podemos usar o Lema de Schur para entender a curvatura de Ricci nos eixos fixos sob um ponto de vista algébrico, a ser explorado na subseção seguinte.

Seja $R$ o tensor de Riemann associado à conexão de Levi-Civita de $g$. Seja $X$ um eixo fixo e considere o operador $R_{X}:=R(\cdot, X) X$. Dado $\bar{Y} \in T_{p} M$, note que

$$
\tilde{\rho}(r) \circ R_{X}(\bar{Y})=R(\tilde{\rho}(r) \bar{Y}, \tilde{\rho}(r) X) \tilde{\rho}(r) X=R(\tilde{\rho}(r) \bar{Y}, X) X=R_{X}(\tilde{\rho}(r) \bar{Y}),
$$

para todo $r \in G_{p}$, onde $\tilde{\rho}: G_{p} \rightarrow O\left(T_{p} M\right)$ consiste na representação de isotropia (isto é, não estamos considerando sua restrição ao espaço horizontal). O Lema de Schur ([SS96, Proposition 4, p.13]) implica que $R_{X}$ consiste em uma matriz diagonal por blocos cujos blocos representam cada subespaço irredutível de $\tilde{\rho}$, precisamente:

Lema 9. Seja $V \subset T_{p} M$ um subespaço $\tilde{\rho}$-irredutivel. Então $\left.R_{X}\right|_{V}$ é um múltiplo da identidade.

Uma vez que $\mathcal{H}_{p}$ é $\tilde{\rho}$-invariante, $R_{X}\left(\mathcal{H}_{p}\right) \subseteq \mathcal{H}_{p}$. Ademais, já que $R_{X}(X)=0, \mathcal{H}_{p} \cap X^{\perp}$ é $R_{X}$-invariante. De agora em diante vamos abusar da notação e denotar por $R_{X}$ a restrição $R_{X}$ : $\mathcal{H}_{p} \cap X^{\perp} \rightarrow \mathcal{H}_{p} \cap X^{\perp}$. Nessa notação, a Proposição 5.2.7 garante que $\operatorname{Ric}_{g}^{\mathcal{H}}(X)=\operatorname{tr} R_{X}$. 
O Corolário 5.2.8 garante que as deformações de Cheeger são inefetivas em $M$ se construirmos uma métrica satisfazendo as condições (1)-(2) no Corolário 5.2.8 junto da condição $\operatorname{Ric}_{g}^{\mathcal{H}}(X)<0$ para algum eixo fixo $X$. Embora seja simples produzir métricas $\operatorname{com} \operatorname{Ric}_{g}^{\mathcal{H}}(X)<0$, a condição (2) impõe maiores restições ao operador $R_{X}$.

Seja $l$ a codimensão de uma órbita regular. Como $\lim _{t \rightarrow \infty} \operatorname{Ric}_{g_{t}}\left(X^{\prime}\right) \geq 1$ para todo $X^{\prime} \in \mathcal{H}$ na parte regular (veja por exemplo a Afirmação 4 ), se $\mathcal{W} \subseteq \mathcal{H}_{p}$ é um $l$-subespaço que é limite de subespaços de espaços horizontais em pontos regulares, então

$$
\operatorname{Ric}_{g}^{\mathcal{W}}(X):=\sum_{i=1}^{l-1} R_{g}\left(X, e_{i}, e_{i}, X\right)=\lim _{t \rightarrow \infty} \sum_{i=1}^{l-1} R_{g_{t}}\left(X, e_{i}, e_{i}, X\right) \geq 1,
$$

onde $\left\{e_{0}=X, e_{1}, \ldots, e_{l-1}\right\}$ é uma base ortonormal para $\mathcal{W}$ e a última igualdade segue do fato que $z_{t}\left(X, e_{i}\right)=0$ para todo $i$ (Veja por exemplo a Proposiçao 5.2.7). Entretanto, o conjunto dos subespaços $\mathcal{W}$ pode ser restrito o bastante de modo que as condições $(5.28)$ e $\operatorname{Ric}_{g}^{\mathcal{H}}(X)<0$ sejam satisfeitas.

Seja $c(s)$ uma curva suave $\operatorname{com} c(0)=p$ tal que $c(s) \in M^{\text {reg }}$ para $s>0$. Para todo $s, \mathcal{H}_{c(s)}$ define uma curva em $\operatorname{Gr}_{l}(T M)$. Qualquer subespaço limite $\mathcal{W}$ obtido ao tomar $s \rightarrow 0^{+}$na curva $\mathcal{H}_{c(s)} \in G r_{l}(T M)$ deve satisfazer a equação (5.28). Podemos concluir que todo subespaço $\mathcal{W}$ surge como limite de espaços horizontais ao longo de uma curva. Chamamos esses subespaços de espaços limites, e denotamos o conjunto desses em um ponto $p$ por $\tilde{\mathcal{W}}_{p}$. Em particular, o Lema 10 fornece a seguinte caracterização para os elementos de $\tilde{\mathcal{W}}_{p}$ :

Lema 10. Seja $\mathcal{W} \in \tilde{\mathcal{W}}_{p}$. Então existe $Y \in \mathcal{H}_{p}$ tal que $\mathcal{W}=\left(\tilde{S}_{Y} \mathfrak{g}_{p}\right)^{\perp}=\left(d \rho\left(\mathfrak{g}_{p}\right) Y\right)^{\perp}$.

Demonstração. Seja $c(s)$ uma curva suave para qual $\mathcal{W}$ seja limite de $\mathcal{H}_{c(s)}$. Se $\epsilon>0$ é pequeno o bastante, seguindo o Lema 8 , uma base para $\mathcal{H}_{c(s)}$ é dada por $\left\{\frac{1}{s} v_{1}^{*}, \ldots, \frac{1}{s} v_{d}^{*}, v_{d+1}^{*}, \ldots, v_{k}^{*}\right\}$, onde $\left\{v_{1}, \ldots, v_{k}\right\}$ é uma base para $\mathfrak{m}_{c(\epsilon)}, v_{1}^{*}, \ldots, v_{d}^{*} \in \mathfrak{g}_{p}$ e $v_{d+1}^{*}, \ldots, v_{k}^{*}$ são vetores $Q$-ortogonais a $\mathfrak{g}_{p}$. Como $v_{i}^{*}(c(s)) \rightarrow 0$ para $i \leq d$, então

$$
\lim _{s \rightarrow 0^{+}} \frac{1}{s} v_{i}^{*}(c(s))=\nabla_{c^{\prime}(0)} v_{i}^{*}=S_{c^{\prime}(0)} v_{i}^{*} .
$$

Isso conclui a demonstração pois $\left\{v_{1}, \ldots, v_{d}\right\}$ deve gerar $\mathfrak{p}_{X}$.

Note portanto que, se $X$ é um eixo fixo, como $d \rho\left(\mathfrak{g}_{p}\right) X=0$, então $X \in \mathcal{W}$ para todo $\mathcal{W} \in \tilde{\mathcal{W}}_{p}$. Em particular, a deformação de Cheeger é ineficiente se $X \in \mathcal{H}_{p}$ satisfizer:

(1') $\operatorname{Ric}_{g}^{\mathcal{H}}(X)<0$

$\left(2^{\prime}\right) \operatorname{Ric}_{g}^{\mathcal{W}}(X) \geq 1$ para todo $\mathcal{W} \in \tilde{\mathcal{W}}_{p}$

Observe que se $\mathcal{H}_{p} \cap X^{\perp}$ é $\rho$-irredutível, a condição (2') junto ao Lema 9, implica que $\operatorname{Ric}^{\mathcal{H}_{p}}(X)>$ 0, o que prova o item (b) no Teorema 5.2.2.

\subsubsection{Uma descrição algébrica do tensor de Ricci em eixos fixos}

Forneceremos uma descrição combinatorial das condições (1'), (2'). Especificamente, obtemos condições algébricas para a existência de um operador simétrico $R_{X}$ satisfazendo $\left(1^{\prime}\right)$ e $\left(2^{\prime}\right)$, para então, a posteriori, construir uma métrica com tal operador de curvatura.

Para evitar problemas técnicos, assumimos o caso em que $\mathcal{H}_{p}=\operatorname{span}\{X\} \oplus \mathcal{H}_{1} \oplus \mathcal{H}_{2}$, onde $\mathcal{H}_{i}$ são subespaços $\rho$-irredutíveis. Nesse caso, $R_{X}$ tem exatamente dois autovalores distintos $\lambda_{1}, \lambda_{2}$. Forneceremos um resultado de existência para $\lambda_{1}, \lambda_{2}$, tal que $\left(1^{\prime}\right),\left(2^{\prime}\right)$ são atendidos.

Observação. 1. Embora o espaço $\mathcal{H}_{p}$ dependa da métrica, a ação lienar de $G_{p}$ em $\mathcal{H}_{p}$ é equivalente à ação clássica da representação de isotropia de $G_{p}$ no quociente $T_{p} M / T_{p} G p$. Portanto, todas as contas podem ser feitas um complemento arbitrário (fixado) de $T_{p} G p$. 
2. A irredutibilidade de $\mathcal{H}_{i}$ é necessária apenas para provar o Teorema 5.2.2. Para produzir exemplos de métricas para as quais as deformações de Cheeger são inefetivas, supomos apenas que $\mathcal{H}_{1}, \mathcal{H}_{2}$ são $\rho$-invariantes. Em ambos os casos, uma vez que os autovalores de $\left.R_{X}\right|_{\mathcal{H}_{1}} e$ $\left.R_{X}\right|_{\mathcal{H}_{2}}$ devem ter sinais diferentes, $\mathcal{H}_{1} \perp \mathcal{H}_{2}$.

Considere as projeções,

$$
p_{i}: \mathcal{H}_{p} \rightarrow \mathcal{H}_{i} .
$$

Então, para qualquer métrica $G$-invariante $g$ e $Y \in \mathcal{H}_{1} \oplus \mathcal{H}_{2}$,

$$
R_{X}(Y)=\lambda_{1}\left|p_{1} Y\right|_{g}^{2}+\lambda_{2}\left|p_{2} Y\right|_{g}^{2}
$$

onde $\lambda_{i}$ são as constantes determinadas pelo Lema de Schur. Em particular,

$$
\operatorname{Ric}^{\mathcal{H}}(X)=\lambda_{1} \operatorname{dim} \mathcal{H}_{1}+\lambda_{2} \operatorname{dim} \mathcal{H}_{2}
$$

Por outro lado, para cada $\mathcal{W} \in \tilde{\mathcal{W}}_{p}$

$$
\operatorname{Ric}^{\mathcal{W}}(X)_{g}=\sum_{i=1}^{l}\left(\lambda_{1}\left|p_{1} e_{i}\right|_{g}^{2}+\lambda_{2}\left|p_{2} e_{i}\right|_{g}^{2}\right)=\lambda_{1} \operatorname{tr}\left(p_{1} \mid \mathcal{W}\right)+\lambda_{2} \operatorname{tr}\left(p_{2} \mid \mathcal{W}\right)
$$

Observação. Embora a definição $\mathcal{W}=\left(d \rho\left(\mathfrak{g}_{p}\right) Y\right)^{\perp}$ seja dependente da métrica, o traço

$$
\operatorname{tr}\left(p_{1} \mid \mathcal{W}\right)=\operatorname{tr}\left(p_{1}\right)-\operatorname{tr}\left(\left.p_{1}\right|_{d \rho\left(\mathfrak{g}_{p}\right) Y}\right)
$$

é independente.

A existência $\lambda_{1}, \lambda_{2}$ satisfazendo $\left(1^{\prime}\right),\left(2^{\prime}\right)$ é totalmente traduzida nas propriedades do conjunto:

$$
\mathcal{A}=\left\{\left(\operatorname{tr}\left(p_{1} \mid \mathcal{W}\right), \operatorname{tr}\left(p_{2} \mid \mathcal{W}\right)\right) \in \mathbb{R}^{2}: \mathcal{W} \in \tilde{\mathcal{W}}_{p}\right\}
$$

Isso nos leva ao seguinte:

Problema 1. Seja $\mathcal{A} \subset \mathbb{R}^{2}$ uma coleção de números reais satsifazendo $a+b=l-1 \forall(a, b) \in \mathcal{A}$. Encontre $\lambda_{1}, \lambda_{2} \in \mathbb{R}$ tais que

$$
a \lambda_{1}+b \lambda_{2} \geq 1, \forall(a, b) \in \mathcal{A}
$$

$e$,

$$
\lambda_{1} \operatorname{dim} \mathcal{H}_{1}+\lambda_{2} \operatorname{dim} \mathcal{H}_{2}<0 .
$$

O Lema 11 fornece condições necessárias e suficientes para resolver o Problema 1.

Lema 11. Denote por $A:=\operatorname{dim} \mathcal{H}_{1}$ e $B:=\operatorname{dim} \mathcal{H}_{2}$. Então o Problema 1 tem solução se, e somente se, ou,

$$
\inf _{(a, b) \in \mathcal{A}}\{a\}>\frac{A(l-1)}{A+B},
$$

ou

$$
\inf _{(a, b) \in \mathcal{A}}\{b\}>\frac{B(l-1)}{A+B},
$$

Demonstração. A partir das condições (5.30), (5.31), fica claro que $\lambda_{1} \lambda_{2}<0$. Além disso, se $\lambda_{1}, \lambda_{2}$ é uma solução para o Problema 1 , então o par $\left(\lambda_{1}^{\prime}, \lambda_{2}^{\prime}\right)=\left(-\lambda_{1},-\lambda_{2}\right)$ também é solução. As duas condições apresentadas no Lema 11 diferem pelo sinal de $\lambda_{1}$. Sem perda de generalidade, assumimos $\lambda_{1}>0$ e provamos (5.32).

Para ver que (5.32) é condição necessária, suponha que o Problema 1 tenha uma solução com $\lambda_{1}>0$. Sejam $(a, b) \in \mathcal{A}$. A equação (5.30) diz que $a>-\frac{\lambda_{2}}{\lambda_{1}} b+\frac{\epsilon}{\lambda_{1}}$ para algum $0<\epsilon<1$, assim, 
$a>(a-(l-1)) \frac{\lambda_{2}}{\lambda_{1}}+\frac{\epsilon}{\lambda_{1}}$, já que $a+b=l-1$. Temos então,

$$
a>(l-1) \frac{-\frac{\lambda_{2}}{\lambda_{1}}}{\left(1-\frac{\lambda_{2}}{\lambda_{1}}\right)}+\frac{\epsilon}{\lambda_{1}-\lambda_{2}} .
$$

Por outro lado, equação (5.31) diz que $\frac{A}{B}<-\frac{\lambda_{2}}{\lambda_{1}}$. Como a função $f(x)=\frac{x}{x+1}$ é crescente em ]0, $\infty[$, concluímos que

$$
a>(l-1) \frac{\frac{A}{B}}{\left(\frac{A}{B}+1\right)}+\frac{\epsilon}{\lambda_{1}-\lambda_{2}}=\frac{(l-1) A}{A+B}+\frac{\epsilon}{\lambda_{1}-\lambda_{2}},
$$

para cada $(a, b) \in \mathcal{A}$, o que prova a equação (5.32).

Suponha agora que exista $\epsilon>0$ tal que

$$
a \geq \frac{A(l-1)+2 \epsilon B}{A+B}
$$

para cada $(a, b) \in \mathcal{A}$. Como $a+b=l-1$, temos $a(A+B)-2 \epsilon B \geq A(a+b)$, assim $\frac{a-2 \epsilon}{b} \geq \frac{A}{B}$ sempre que $b \neq 0$. Escolhendo $\lambda_{1}, \lambda_{2}$ tais que $\lambda_{1}>0$ e $\frac{a-\epsilon}{b} \geq-\frac{\lambda_{2}}{\lambda_{1}}>\frac{A}{B}$, obtemos $0>\lambda_{1} A+\lambda_{2} B$ e $\lambda_{1} a+\lambda_{2} b \geq \epsilon$ para cada $(a, b) \in \mathcal{A}, b \neq 0$. O Lema segue após um escalonamento pois, quando $\lambda_{1}>0$, a equação (5.30) é automaticamente satisfeita para $b=0$.

A seguinte proprosição pode ser entendida como uma recíproca do Teorema 5.2.2.

Proposição 5.2.9. Suponha que $\mathcal{H}_{p}$ tenha um subespaço $\rho$-invariante $\mathcal{H}_{1}$ tal que $X \notin \mathcal{H}_{1}$ e que

$$
\inf _{\mathcal{W} \in \tilde{\mathcal{W}}_{p}}\left\{\operatorname{tr}\left(p_{1} \mid \mathcal{W}\right)\right\}>\frac{\operatorname{dim} \mathcal{H}_{1}(l-1)}{\operatorname{dim} \mathcal{H}_{p}-1} .
$$

Então existe uma métrica $G$-invariante $g$ em uma vizinhança $U \ni p$ satisfazendo:

1. $\operatorname{Ric}_{U^{r e g} / G} \geq 1$,

2. $\operatorname{Ric}_{g_{t}}(X)<0$ para toda deformação de Cheeger $g_{t}$ de $g$.

Demonstração. Uma vez que a equação (5.34) é verificada, a existência de uma métrica satisfazendo

$$
R_{X}=\lambda_{1} p_{1}+\lambda_{2} p_{2},
$$

onde $\lambda_{1}, \lambda_{2}$ são dados pela Lema (11) seguirá a partir do uso de produtos verticais.

De fato, considere uma métrica bi-invariante $Q$ em $G$ e recorde que exista uma vizinhança $G$ invariante de $p$ que é equivariantemente difeomorfa a $G \times{ }_{\rho} \mathcal{H}_{p}$. É portanto suficiente definir uma métrica $\rho\left(G_{p}\right)$-invariante em $\mathcal{H}_{p}$ tal que $R_{X}=\lambda_{1} p_{1}+\lambda_{2} p_{2}$ e, para algum $c>0$, $\operatorname{Ric}^{\mathcal{W}} \geq c$ para todo $\mathcal{W} \in \tilde{\mathcal{W}}_{p}$.

Escreva $\mathcal{H}_{p} \cong \mathbb{R} \times \mathbb{R}^{n_{1}} \times \mathbb{R}^{n_{2}}$, onde $\mathbb{R}$ corresponde ao eixo fixo e $\mathbb{R}^{n_{1}}, \mathbb{R}^{n_{2}}$ aos dois subespaços $\rho\left(G_{p}\right)$-invariantes $\mathcal{H}_{1}, \mathcal{H}_{2}$, respectivamente. Considere a métrica

$$
\bar{g}=d t^{2}+\phi^{2}(t) d s_{\mathbb{R}^{n_{1}}}^{2}+\psi^{2}(t) d s_{\mathbb{R}^{n_{2}}}^{2},
$$

onde $d s_{\mathbb{R}^{n_{i}}}^{2}$ é a métrica padrão (plana) de $\mathbb{R}^{n_{i}} \mathrm{e}$

$$
\begin{aligned}
& \phi(t)=\frac{1}{\sqrt{\lambda_{1}}} \sin \left(\sqrt{\lambda_{1}} t\right), \\
& \psi(t)=\frac{1}{\sqrt{-\lambda_{2}}} \exp \left(\sqrt{-\lambda_{2}} t-b\right),
\end{aligned}
$$

para algum $b>0$ a ser escolhido. Seguindo a notação de [Pet06b, page 71], dados $V, V^{\prime} \in$ 
$T \mathbb{R}^{n_{1}}, W, W^{\prime} \in T \mathbb{R}^{n_{2}}$, temos

$$
\begin{aligned}
\Re_{\bar{g}}(X \wedge V) & =\lambda_{1} X \wedge V, \\
\Re_{\bar{g}}(X \wedge W) & =\lambda_{2} X \wedge W, \\
\Re_{\bar{g}}\left(V \wedge V^{\prime}\right) & =\lambda_{1} V \wedge V^{\prime}, \\
\Re_{\bar{g}}\left(W \wedge W^{\prime}\right) & =-\lambda_{2}\left(\exp \left(b-\sqrt{-\lambda_{2}} t\right)^{2}-1\right) W \wedge W^{\prime}, \\
\Re_{\bar{g}}(V \wedge W) & =-\sqrt{-\lambda_{1} \lambda_{2}} \cot \left(\sqrt{\lambda_{1}} t\right) V \wedge W,
\end{aligned}
$$

onde $\Re_{\bar{g}}$ é o tensor de curvatura de $\bar{g}$ e $X \wedge Y(v)=g(Y, v) X-g(X, v) Y$. Em particular, $R_{X}=$ $\lambda_{1} p_{1}+\lambda_{2} p_{2}$, como desejado. Para verificar que $\operatorname{Ric}^{\mathcal{W}} \geq c$ em $p$, basta identificar $\mathbb{R} \times \mathbb{R}^{n_{1}} \times \mathbb{R}^{n_{2}}$ com $\mathcal{H}_{p}$ de uma maneira que $p$ corresponda ao ponto $\left(t_{0}, 0,0\right)$, onde

$$
-\sqrt{-\lambda_{1} \lambda_{2}} \cot \left(\sqrt{\lambda_{1}} t_{0}\right) \geq \max \left\{\lambda_{1},\left(1-\lambda_{2}\right) \frac{A+B}{A(l-1)}\right\} .
$$

Escolhendo $b>\sqrt{-\lambda_{2}} t_{0}$ e usando (5.38)-(5.42), concluímos que dado $\alpha X+V+W \in \mathcal{W}$,

$$
\operatorname{Ric}^{\mathcal{W}}(\alpha X+V+W)=\alpha^{2} \operatorname{Ric}^{\mathcal{W}}(X)+\operatorname{Ric}^{\mathcal{W}}(V)+\operatorname{Ric}^{\mathcal{W}}(W)
$$

Note que $\operatorname{Ric}^{\mathcal{W}}(X) \geq 1$ pelo Lema 11 e que $\operatorname{Ric}^{\mathcal{W}}(V) \geq \lambda_{1}|V|^{2}$ (veja as equações (5.38),(5.40) e (5.42)). Assim, $\operatorname{Ric}^{\mathcal{W}}(W)$ satisfaz

$$
\operatorname{Ric}^{\mathcal{W}}(W) \geq K(X, W)+\operatorname{tr}\left(p_{1} \mid \mathcal{W}\right)\left(-\sqrt{-\lambda_{1} \lambda_{2}} \cot \left(\sqrt{\lambda_{1}} t_{0}\right)\right)|W|^{2} \geq \lambda_{1}|W|^{2}
$$

já que $\operatorname{tr}\left(p_{1} \mid \mathcal{W}\right) \geq \frac{A(l-1)}{A+B}$ e $t_{0}$ satisfaz (5.43). Em particular, após um re-escalonamento apropriado da métrica, a equação (5.35) implica a prova da Proposição.

A seguir nós fornecemos exemplos globais onde a deformação de Cheeger é não efetiva. A prova do Teorema 5.2.2 estará completa ao fim dessa seção, onde provaremos que:

$$
\inf _{\mathcal{W} \in \tilde{\mathcal{W}}_{p}}\left\{\operatorname{tr}\left(p_{1} \mid \mathcal{W}\right)\right\}=\operatorname{dim} \mathcal{H}_{1}-\operatorname{dim} \rho\left(G_{p}\right) Y_{1}
$$

\subsubsection{Uma família de contra-exemplos}

Aplicamos o Lema 11 para provar o Teorema 5.2.4. Nosso exemplo modelo consiste numa métrica de produto vertical duplo na esfera $S^{5} \subset \mathbb{R}^{6}$, munida da ação mono-axial usual de $S O(3)$.

1. Considere $S^{5} \subset \mathbb{R}^{6}$ munida da ação mono-axial padrão por $S O(3)$, isto é, a ação que fixa as três primeiras coordenadas. Tome $p=(1,0,0,0,0,0), X=(0,1,0,0,0,0)$ e note que $G_{p}=G_{X}=S O(3)$.

2. As órbitas regulares dessa ação são difeomorfas à esferas de dimensão 2. Portanto, a dimensão $l$ do espaço horizontal em pontos regulares é 3 .

Denote por $\mathcal{H}_{p}=\operatorname{span}\{X\} \oplus \mathcal{H}_{1} \oplus \mathcal{H}_{2}$, a decomposição $\rho$-invariante onde $\mathcal{H}_{1}=\operatorname{span}\{(0,0,1,0,0,0)\}$ e $\mathcal{H}_{2}$ é dado pelas três últimas coordenadas. Então, $A=1$ e $B=3$ no Lema 11. Mostraremos que $\operatorname{tr}\left(p_{1} \mid \mathcal{W}\right) \geq 1>\frac{1}{2}$ para todo $\mathcal{W} \in \tilde{\mathcal{W}}_{p}$

De fato, dado $Y \in \mathcal{H}_{1} \oplus \mathcal{H}_{2}, \rho(S O(3)) Y \subset \mathcal{H}_{2}$, portanto $(0,0,1,0,0,0) \in(d \rho(\mathfrak{s o}(3)) Y)^{\perp}$ para todo $Y$. Usando o Lema 10, concluímos que $\operatorname{tr}\left(p_{1} \mid \mathcal{W}\right)=1$ para todo $\mathcal{W}$. Assim, o Lema 11 garante a existência de $\lambda_{1}, \lambda_{2}$ tais que, se $g$ satisfaz $\left.R_{X}\right|_{\mathcal{H}_{i}}=\lambda_{i} e$, então $\operatorname{Ric}_{g_{t}}(X)<0$ para todo $t$ grande. Considere então a métrica

$$
g=d t^{2}+\phi^{2}(t) d s_{S^{2}}^{2}+\psi^{2}(t) d s_{S^{2}}^{2},
$$


onde $t \in\left(\frac{\pi}{2 \sqrt{\lambda_{1}}}, \frac{\pi}{\sqrt{\lambda_{1}}}\right) ; \phi$ é como em (5.36); $\psi$ é como em (5.37) (com $b$ arbitrário) para $t \in$ $\left(\frac{\pi}{2 \sqrt{\lambda_{1}}}, \frac{\pi}{2 \sqrt{\lambda_{1}}}+\epsilon\right)$ e arbitrário em $\left[\frac{\pi}{2 \sqrt{\lambda_{1}}}+\epsilon, \frac{\pi}{\sqrt{\lambda_{1}}}\right)$ (desde que as funções definam uma métrica suave em $S^{5}$ ). Aqui, o primeiro $d s_{S^{2}}^{2}$ está relacionado às três primeiras coordenadas em $S^{5}$ e o segundo às três últimas coordenadas. Os pontos fixos correspondem à $t=\pi / 2 \sqrt{\lambda_{1}}$ que, por continuidade da métrica, tem $R_{X}=\lambda_{1} p_{1}+\lambda_{2} p_{2}$. Mais ainda, a ação é transitiva no segundo $S^{2}$ e seu espaço quociente é exatamente $S^{5} / S O(3)$, isto é, um disco com a métrica de produto vertical

$$
\tilde{g}=d t^{2}+\frac{1}{\lambda_{1}} \sin \left(\sqrt{\lambda_{1}} t\right)^{2} d s_{S^{2}}^{2},
$$

que tem curvatura constante e positiva. A esfera $S^{5}$ com um re-escalonamento de (5.44) é nosso primeiro exemplo de $G$-variedade cujas órbitas têm grupo fundamental finito, cujo quociente satisfaz $\operatorname{Ric}_{M / G} \geq 1$ e tal que a deforamação de Cheeger é inefetiva.

Para dimenõses mais altas, considere $S^{n}$ com a ação padrão mono-axial de $S O(n-2)$-fixando as três primeiras coordenadas. Tome $p=(1,0,0, \ldots, 0), X=(0,1,0, \ldots, 0), \mathcal{H}_{1}=\operatorname{span}\{(0,0,1,0, \ldots, 0)\}$ e $\mathcal{H}_{2}$ seu complemento ortogonal na métrica padrão de $\mathbb{R}^{n+1}$. Note que as órbitas regulares têm dimeãnso $n-3$. Procedendo exatamente da mesma maneira que no caso de $S^{5}$ prova-se que

$$
\operatorname{tr}\left(p_{1} \mid \mathcal{W}\right)=1>\frac{(l-1) \operatorname{dim} \mathcal{H}_{1}}{\operatorname{dim} \mathcal{H}_{p}-1}=\frac{2}{n-1}
$$

para todo $\mathcal{W} \in \tilde{\mathcal{W}}_{p}$. Com as mesmas funções para o produto vertical, temos que

$$
g=d t^{2}+\phi^{2}(t) d s_{S^{2}}^{2}+\psi^{2}(t) d s_{S^{n-3}}^{2},
$$

satisfaz todas as propriedades desejadas.

\subsubsection{Um critério algébrico para não positividade da curvatura de Ricci}

Para completar a demonstração do Teorema 5.2.2, fixe um produto interno $\rho$-invariante em $\mathcal{H}_{p}$ e seja $\mathcal{R}$ a parte regular de $\mathcal{H}_{p}$ com respeito à $\rho$. Provamos que:

Proposição 5.2.10. Suponha que $d \rho\left(\mathfrak{g}_{p}\right) X=0$ e que $\mathcal{H}_{p}=\operatorname{span}\{X\} \oplus \mathcal{H}_{1} \oplus \mathcal{H}_{2}$ seja uma decomposição $\rho$-invariante. Para qualquer $Y \in \mathcal{R}$, denote por $Y_{i}$ a componente de $Y$ pertencente ao subespaço $\mathcal{H}_{i}$. Então

$$
\inf _{\mathcal{W} \in \tilde{\mathcal{W}}_{p}}\left\{\operatorname{tr}\left(p_{1} \mid \mathcal{W}\right)\right\}=\inf _{Y \in \mathcal{R}}\left\{\operatorname{dim} \mathcal{H}_{1}-\operatorname{dim} \rho\left(G_{p}\right) Y_{1}\right\}
$$

Demonstração. Fixe $Y=Y_{1}+Y_{2} \in \mathcal{R}$. Para todo $\alpha>0$, defina $\mathcal{W}^{\alpha}=\left(d \rho\left(\mathfrak{g}_{p}\right)\left(\alpha Y_{1}+Y_{2}\right)\right)^{\perp}$. É suficiente mostrar que:

$$
\inf _{\alpha>0}\left\{\operatorname{tr}\left(p_{1} \mid \mathcal{W}^{\alpha}\right)\right\}=\operatorname{dim} \mathcal{H}_{1}-\operatorname{dim} \rho\left(G_{p}\right) Y_{1}
$$

Escreva $\mathcal{W}^{\alpha}=\mathcal{W}_{1} \oplus \mathcal{W}_{2} \oplus \mathcal{W}_{12}^{\alpha}$ onde

$$
\begin{aligned}
\mathcal{W}_{1} & :=\operatorname{ker} p_{2} \cap \mathcal{W}^{\alpha}, \\
\mathcal{W}_{2} & :=\operatorname{ker} p_{1} \cap \mathcal{W}^{\alpha}, \\
\mathcal{W}_{12}^{\alpha} & :=\mathcal{W}^{\alpha} \cap\left(\mathcal{W}_{1} \oplus \mathcal{W}_{2}\right)^{\perp}
\end{aligned}
$$

sendo $p_{i}$ a projeção em $\mathcal{H}_{i}$. Note que $\mathcal{W}_{1}, \mathcal{W}_{2}$ não dependem de $\alpha$, já que $\mathcal{W}_{i}=\left\{Z_{i} \in \mathcal{H}_{i} \mid Z_{i} \perp\right.$ $\left.d \rho\left(\mathfrak{g}_{p}\right) Y_{i}\right\}$. Observe também que a dimensão de $\mathcal{W}_{12}^{\alpha}$ não depende de $\alpha$ e, ainda mais:

Lema 12. Existem constantes $c, C>0$, não dependendo de $\alpha$, tais que

$$
\frac{1}{1+c \alpha^{2}} \leq \frac{\left|p_{1}\left(v_{\alpha}\right)\right|^{2}}{\left|v_{\alpha}\right|^{2}} \leq \frac{1}{1+C \alpha^{2}}
$$


para todos $v_{\alpha} \in \mathcal{W}_{12}^{\alpha}$.

Demonstração. Seja $\mathcal{M}=d \rho\left(\mathfrak{g}_{p}\right) Y_{1} \oplus d \rho\left(\mathfrak{g}_{p}\right) Y_{2}$ e note que $\mathcal{W}_{12}^{\alpha} \subset \mathcal{M}$. Seja $\mathcal{M}_{12}^{\alpha}$ o complemento ortogonal de $W_{12}^{\alpha}$ em $\mathcal{M}$. Note que $\left.p_{1}\right|_{\mathcal{M}_{12}^{\alpha}},\left.p_{2}\right|_{\mathcal{M}_{12}^{\alpha}}$ são isomorfismos sobre suas imagens. Precisamente, um elemento no kernel de $\left.p_{1}\right|_{\mathcal{M}_{12}^{\alpha}}$ pertence a $\mathcal{H}_{2}$ e é ortogonal $\mathcal{W}_{2}$ e $\mathcal{W}_{12}^{\alpha}$. Isto é, $\left.v \in \operatorname{ker} p_{1}\right|_{\mathcal{M}_{12}^{\alpha}} \subset \mathcal{H}_{2} \cap\left(\mathcal{W}_{12}^{\alpha}\right)^{\perp} \cap \mathcal{W}_{2}^{\perp}$. Entretanto, $p_{2}\left(\mathcal{W}_{2}+\mathcal{W}_{12}^{\alpha}\right)=\mathcal{H}_{2}$, de onde $v=0$. Um argumento análogo verifica a afirmação correspondente para $\left.p_{2}\right|_{\mathcal{M}_{12}^{\alpha}}$. Mais ainda, dado $Z_{1}+Z_{2} \in \mathcal{M}$, temos que $\alpha Z_{1}+Z_{2} \in \mathcal{M}_{12}^{\alpha}$. Assim, existe um mapa linear e invertível $T: p_{1}(\mathcal{M}) \rightarrow p_{2}(\mathcal{M})$ tal que

$$
Z \in \mathcal{M}_{12}^{\alpha} \Leftrightarrow Z=\alpha Z_{1}+T\left(Z_{1}\right) ; \text { para algum } Z_{1} \in p_{1}(\mathcal{M}) .
$$

Daí,

$$
W \in \mathcal{W}_{12}^{\alpha} \Leftrightarrow W=W_{1}-\alpha\left(T^{*}\right)^{-1} W_{1} ; \text { para algum } W_{1} \in p_{1}(\mathcal{M}) .
$$

Como $|T|^{-1} \leq\left|T^{-1}\right|$ e $|T|=\left|T^{*}\right|$ temos

$$
\left|W_{1}\right|^{2}+\alpha^{2}|T|^{-2}\left|W_{1}\right|^{2} \leq|W|^{2} \leq\left|W_{1}\right|^{2}+\alpha^{2}\left|T^{-1}\right|^{2}\left|W_{1}\right|^{2} .
$$

Estimamos agora $\operatorname{tr}\left(p_{1} \mid \mathcal{W}^{\alpha}\right)$. Como $\mathcal{W}_{2} \subset \operatorname{ker} p_{1}$, tome bases ortonormais $\left\{e_{1}, \ldots, e_{d_{1}}\right\}$ e $\left\{e_{1}^{\alpha}, \ldots, e_{d}^{\alpha}\right\}$ para $\mathcal{W}_{1}$ e $\mathcal{W}_{12}^{\alpha}$, respectivamente. Note que

$$
\operatorname{tr}\left(p_{1} \mid \mathcal{W}^{\alpha}\right)=\sum_{j}^{d_{1}}\left\langle p_{1} e_{j}, e_{j}\right\rangle+\sum_{k}^{d}\left\langle p_{1} e_{k}^{\alpha}, e_{k}^{\alpha}\right\rangle=\operatorname{dim} \mathcal{W}_{1}+\sum_{k}^{d}\left|p_{1} e_{k}^{\alpha}\right|^{2}
$$

O Lema 12 diz que:

$$
\operatorname{dim} \mathcal{W}_{1}+\frac{1}{1+c \alpha} \operatorname{dim} \mathcal{W}_{12}^{\alpha} \leq \operatorname{tr}\left(p_{1} \mid \mathcal{W}^{\alpha}\right) \leq \operatorname{dim} \mathcal{W}_{1}+\frac{1}{1+C \alpha} \operatorname{dim} \mathcal{W}_{12}^{\alpha}
$$

Tomando $\alpha \rightarrow \infty$ concluímos que $\inf _{\alpha}\left\{\operatorname{tr}\left(p_{1} \mid \mathcal{W}^{\alpha}\right)\right\}=\operatorname{dim} \mathcal{W}_{1}$. Por outro lado, $\mathcal{W}_{1}=\left(d \rho\left(\mathfrak{g}_{p}\right) Y_{1}\right)^{\perp} \cap$ $\mathcal{H}_{1}$, e portanto, $\operatorname{dim} \rho\left(G_{p}\right) Y_{1}+\operatorname{dim} \mathcal{W}_{1}=\operatorname{dim} \mathcal{H}_{1}$, o que conclui a demonstração.

\subsection{Um refinamento para o Teorema de Lawson-Yau}

Lawson e Yau provaram em [LS74] que qualquer $G$-variedade riemanniana compacta e conexa $(M, G)$, com $G$ não-abeliano, admite uma métrica de curvatura escalar positiva. Essa é obtida predefinindo a métrica em pontos singulares e encolhendo as órbitas da ação por $G$ via aplicação de uma deformação canônica, o que resulta na necessidade de complicadas estimativas em pontos singulares. De mangeira geral, em sua demonstração, a técnica necessita a redução grupo de estrutura $G$ para $S O(3)$ ou $S^{3}$ e a métrica resultante não necessariamente possui semelhanças com a métrica original. Especialmente em pontos singulares.

Como consequência do entendimento do tensor $z_{t}$ associado às deformações de Cheeger, uma vez que a curvatura escalar leva em conta a curvatura seccional de todos os dois-planos em $\operatorname{Gr}_{2}(T M)$, conseguimos uma demonstração muito mais simples e natural para esse resultado, que preserva, em particular, o grupo de estrutra. Precisamente:

Teorema 5.3.1 (Refinamento para o Teorema de Lawson-Yau). Seja $(M, g)$ uma G-variedade riemanniana e assuma que $G$ seja não-abeliano. Então, $g$ desenvolve curvatura escalar positiva após deformação de Cheeger finita. 
Demonstração do Teorema 5.3.1. A demonstração se dá por contradição. Assuma que para cada $n \in \mathbb{N}$ exista $p_{n} \in M$ tal que

$$
\operatorname{scal}_{g_{n}}\left(p_{n}\right) \leq 0 .
$$

Como $M$ é compacta, podemos passar a uma subsequência, se necessário, e extrair um ponto limite $p \in M$ tal que

$$
\operatorname{scal}_{g_{\infty}}(p) \leq 0 .
$$

Entretanto, como $G$ é não-abeliano, pela expressão (5.15), necessariamente $p$ deve ser um ponto fixo. Por outro lado, pelo Lema 8, existe ao menos um par $e_{i}, e_{j}$ no espaço horizontal de $p$ tal que pela Proposição 5.2.6 devemos ter $z_{t}\left(e_{i}, e_{j}\right) \rightarrow \infty$, o que é uma contradição com a equação (5.50).

\subsection{Uma demonstração simplificada do Teorema de Searle-Wilhelm}

O objetivo desta seção é fornecer uma prova simplificada para o Teorema 5.2.1 (Teorema C).

Teorema 5.4.1. Seja $(M, g)$ uma $G$-variedade riemanniana. Assuma que $(M, g), G$ sejam como nas hipóteses do Teorema 5.2.1. Então existe uma função suave e $G$-invariante $f: M \rightarrow \mathbb{R}$ tal que a métrica $\tilde{g}=g e^{2 f}$ possui curvatura horizontal de Ricci positiva em uma vizinhança do estrato singular, e ainda, métrica de submersão por essa induzida na variedade $M^{\text {reg }} / G$ é tal que $\operatorname{Ric}_{M^{\text {reg }} / G} \geq 1$.

Demonstração. Usando o Teorema de Slice 3.1.10, lembre-se que todo ponto singular $q$ tem uma vizinhança $U_{q}$ tal que para qualquer $q^{\prime} \in U_{q}, G_{q^{\prime}}$ é conjugado a um subgrupo de $G_{q}$. Defina

$$
S_{q}:=\operatorname{Fix}\left(G_{q}\right):=\left\{q^{\prime} \in M \mid G_{q} \subseteq G_{q^{\prime}}\right\} .
$$

Para cada ponto singular $q, S_{q}$ é uma subvariedade totalmente geodésica de $M$. Afirmamos que $G S_{q}$ tem interior relativo não vazio para cada $q$ no estrato singular. Precisamente, sejam $q_{0} \in G S_{q}$ e $U_{q}$ uma vizinhança de $q$ tal que para quaisquer $q^{\prime} \in U_{q}$ exista $g \in G$ tal que $g G_{q^{\prime}} g^{-1} \subseteq G_{q}$.

Como $q_{0} \in G S_{q}$, então existem $h \in G$ e $q_{0}^{\prime} \in S_{q}$ de modo que $q_{0}=h q_{0}^{\prime}$. Em particular,

$$
h^{-1} G_{q_{0}} h=G_{h^{-1} q_{0}}=G_{q_{0}^{\prime}} \supseteq G_{q} .
$$

Daí,

$$
G_{q_{0}} \supseteq h G_{q} h^{-1} .
$$

Logo, se $q^{\prime} \in U_{q}$ então a menos de conjugação, $G_{h q^{\prime} h^{-1}} \subseteq G_{q_{0}}$, de onde segue o afirmado.

Uma vez que o estrato singular $S$ é também compacto, existe um conjunto finito de pontos $\widehat{M} \subset M$ tal que

$$
\bigcup_{q \in \widehat{M}} G S_{q}=S
$$

Para cada $q$, considere a função

$$
\psi_{q}:=\operatorname{dist}^{2}\left(\cdot, S_{q}\right),
$$

e seja $\rho$ uma função cuja segunda derivada satisfaz:

$$
\rho^{\prime \prime}:=\left\{\begin{array}{l}
\frac{2}{\epsilon}-\frac{2}{\epsilon^{2}} t, t \in[0, \epsilon], \\
0, t \geq \epsilon,
\end{array}\right.
$$

e ainda, $\rho^{\prime}(0)=-1 ; \rho(0)$ é tal que $\rho(\epsilon)=0$ e $\epsilon$ será escolhido depois. Note que a integral de $\rho^{\prime \prime}$ é 1 , então $\rho^{\prime} \leq 0$ para todo $t$ e $\rho$ é constante para $t \geq \epsilon$. Afirmamos que existe $b$ tal que a função

$$
f(p)=b \sum_{q} \int_{G} \rho \circ \psi_{q}(g p) d g
$$


é a função que realizará a tese.

De fato, lembre-se que a fórmula para curvatura seccional em uma deformação conforme $\tilde{g}=e^{2 f} g$ é dada por (veja [Wal04, p. 143-144])

$$
\sec _{\tilde{g}}(X, Y)=e^{-2 f}\left(\sec _{g}(X, Y)-h_{f}(X)-h_{f}(Y)-|\nabla f|^{2}+d f(X)^{2}+d f(Y)^{2}\right),
$$

onde $X, Y$ são $g$-ortonormais. Fixando uma base $g$-ortonormal $\left\{e_{1}, \ldots, e_{n}\right\}$ para $\mathcal{H}$, obtemos:

$$
\begin{aligned}
\operatorname{Ric}_{\tilde{g}}^{\mathcal{H}}(Z)=e^{-2 f}\left(\operatorname{Ric}_{g}^{\mathcal{H}}(Z)-(n-1) h_{f}(Z)-\sum_{i=1}^{n-1}\right. & h_{f}\left(e_{i}\right) \\
& \left.\quad+(n-1)\left\{-|\nabla f|^{2}+d f(Z)^{2}\right\}+\sum_{i=1}^{n-1} d f\left(e_{i}\right)^{2}\right) .
\end{aligned}
$$

Note que sempre podemos escolher $e_{1}=Z=\alpha e_{1}^{\prime}+\beta e_{2}^{\prime}, \alpha^{2}+\beta^{2}=1$, onde $e_{1}^{\prime}=\nabla f /|\nabla f|, e_{2}^{\prime}$ é ortonormal a $e_{1}^{\prime}$ e $\left\{e_{1}=Z, e_{2}:=-\beta e_{1}^{\prime}+\alpha e_{2}^{\prime}, e_{3}, \ldots, e_{n}\right\}$ é ortonormal. Assim $d f\left(e_{2}^{\prime}\right)=d f\left(e_{i}\right)=0$ para $i \geq 3$. Portanto,

$$
-|\nabla f|^{2}+d f(Z)^{2}=-\sum_{i=2}^{n} d f\left(e_{i}\right)^{2}=-\beta^{2}|\nabla f|^{2} .
$$

Logo,

$$
\operatorname{Ric}_{\tilde{g}}^{\mathcal{H}}(Z)=e^{-2 f}\left(\operatorname{Ric}_{g}^{\mathcal{H}}(Z)-(n-1) h_{f}(Z)-\sum_{i=2}^{n} h_{f}\left(e_{i}\right)-(n-2) \beta^{2}|\nabla f|^{2}\right) .
$$

Por outro lado,

$$
h_{f}(W)=\sum_{q} \int_{G} h_{\rho \circ \psi_{q}}(g W) d g=\sum_{q} \int_{G}\left\{\rho^{\prime \prime} d \psi_{q}(g W)^{2}+\rho^{\prime} h_{\psi_{q}}(g W)\right\} d g .
$$

Logo,

$$
\begin{aligned}
e^{2 f} \operatorname{Ric}_{\tilde{g}}^{\mathcal{H}}(Z)=\operatorname{Ric}_{g}^{\mathcal{H}}(Z)-(n-2) \beta^{2}|\nabla f|^{2} & \\
& -\int_{G} \sum_{q} b \rho^{\prime \prime}\left((n-1) d \psi_{q}(g Z)^{2}+\sum_{i=1}^{n-1} d \psi_{q}\left(g e_{i}\right)^{2}\right) d g \\
& \quad-\int_{G} \sum_{q} b \rho^{\prime}\left((n-1) h_{\psi_{q}}(g Z)+\sum_{i=1}^{n-1} h_{\psi_{q}}\left(g e_{i}\right)\right) d g,
\end{aligned}
$$

Uma vez que $\left.d \psi_{q}\right|_{S_{q}}=0$ e $S_{q}$ é totalmente geodésica, existe uma vizinhança de $S_{q}$ tal que $d \psi_{q}(X)^{2} \leq 4|X|_{g}^{2} \psi_{q}(x)$. Como $\rho^{\prime \prime} \leq 2 / \epsilon$ temos

$$
\rho^{\prime \prime}\left((n-1) d \psi_{q}(g Z)^{2}+\sum_{i=1}^{n-1} d \psi_{q}\left(g e_{i}\right)^{2}\right) \leq \rho^{\prime \prime}(n-1) 4 \psi_{q} \leq 8(n-1) \epsilon .
$$

Mais ainda,

$$
|\nabla f|^{2}=\sum_{q \in \widehat{M}} \int_{G} b^{2}\left(\rho^{\prime}\right)^{2}\left|\nabla \psi_{q}\right|^{2} \leq \epsilon^{2} b^{2}|\widehat{M}| .
$$

Como $S_{q}$ é totalmente geodésica, $h_{\psi_{q}}(X)=|X|^{2}$ para todo $X$ normal a $S_{q}$ e $h_{\psi_{q}}(X)=0$ para $X$ tangente a $S_{q}$. Portanto, dado $c>0$, podemos escolher $\epsilon$ e $e_{3}, \ldots, e_{n}$ tais que ou $h_{\psi_{q}}\left(e_{i}\right) \geq 1-c \psi_{q}$ ou $h_{\psi_{q}}\left(e_{i}\right) \geq-c \psi_{q}$. Mais ainda, $h_{\psi_{q}}(\nabla f) \geq|\nabla f|^{2}(1-c)$. 
Temos portanto que

$$
\begin{aligned}
(n-1) h_{\psi_{q}}(Z)+\sum_{i=2}^{n} h_{\psi_{q}}\left(e_{i}\right) & \geq(n-1) \alpha^{2}+\beta^{2}-(2 n+2) c \\
& \geq 1-(2 n+2) c .
\end{aligned}
$$

Aplicando (5.55)-(5.56) à (5.54) obtemos

$$
e^{2 f} \operatorname{Ric}_{\tilde{g}}^{\mathcal{H}}(Z) \geq \operatorname{Ric}_{g}^{\mathcal{H}}(Z)-(n-2) \beta^{2} b^{2}|\widehat{M}| \epsilon^{2}-8 b|\widehat{M}|(n-1) \epsilon-b \int_{G} \sum_{q} \rho^{\prime} d g(1-(2 n+2) c) .
$$

Já que $\rho^{\prime}$ é uma função não positiva tal que $\rho^{\prime}(0)=-1$, então $\int_{G} \sum_{q} \rho^{\prime} \circ \psi_{q}(g p)<0$ para todo $p \in S$. Logo, para cada $\delta>0$, pode-se escolher $b$ e $\epsilon$ tais que (5.57) é positiva para todo vetor unitário $\left.Z \in \mathcal{H}\right|_{S}$. Ademais,

$$
(n-2) \beta^{2} b^{2}|\widehat{M}| \epsilon^{2}+8 b|\widehat{M}|(n-1) \epsilon \leq \delta .
$$

Isso conclui a demonstração uma vez que $\operatorname{Ric}_{g}^{\mathcal{H}}(Z) \geq 1$ para todo $\left.Z \in \mathcal{H}\right|_{M \backslash S}$.

Demonstração do Teorema 5.2.1. Assuma que $(M, g)$ e $G$ sejam como nas hipóteses do Teorema 5.2.1. A prova pode ser dividida levando em conta a existência, ou não, de um ponto singular $p \in M$ e um vetor não nulo $X \in \mathcal{H}_{p}$ fixado pela representação de isotropia.

Assuma que para todo ponto singular $p \in M$ o único vetor fixado pela represtanção de isotropia em $\mathcal{H}_{p}$ seja o vetor nulo. Então, pelo Corolário 5.2.3, existe $t>0$ tal que a deformação de Cheeger $g_{t}$ de $g$ possui curvatura de Ricci positiva, o que demonstra o teorema nesse caso.

Suponha por outro lado que exista um ponto singular $p \in M$ e um vetor não nulo $X \in \mathcal{H}_{p}$, fixado pela representação de isotropia em $\mathcal{H}_{p}$. Considere em $M$ a métrica $\widetilde{g}$ obtida no Teorema 5.4.1. Então, pelo Corolário 5.2.8 existe $t>0$ tal que a deformação de Cheeger $\widetilde{g}_{t}$ de $\widetilde{g}$ tem curvatura de Ricci positiva, o que conclui a demonstração do Teorema.

\subsection{Deformações de Cheeger aplicadas à diagramas estrela}

Nesta seção provamos os seguintes resultados:

Teorema 5.5.1. Sejam $\Sigma^{7}$ e $\Sigma^{8}$ quaisquer variedades fechadas homotópicas à esferas padrões de dimensões 7 e 8, respectivamente; $\Sigma^{10}$ qualquer variedade fechada homotópica à 10-esfera e que seja bordo de uma variedade spin; $\Sigma^{4 m+1}, \Sigma^{8 m+5}$ esferas de Kervaire em dimensões $4 m+1,8 m+5$, respectivamente. Então cada variedade abaixo admite uma sequência de métricas $\left\{g_{n}\right\}_{n \in \mathbb{N}}$ com curvatura de Ricci positiva satisfazendo $\sec _{g_{n}} \geq-1 / n$ e $\operatorname{diam}_{g_{n}} \leq 1 / n$ :

(i) $M^{7} \# \Sigma^{7}$, onde $M^{7}$ é qualquer $S^{3}$-fibrado sobre $S^{4}$

(ii) $M^{8} \# \Sigma^{8}$, onde $M^{8}$ é ou um $S^{3}$-fibrado sobre $S^{5}$ ou um $S^{4}$-fibrado sobre $S^{4}$

(iii) $M^{10} \# \Sigma^{10}$, onde:

(a) $M^{10}=M^{8} \times S^{2}$ com $M^{8}$ como no item (ii)

(b) $M^{10}$ é qualquer $S^{3}$-fibrado sobre $S^{7}, S^{5}$-fibrado sobre $S^{5}$ ou $S^{6}$-fibrado sobre $S^{4}$

(iv) $M^{4 m+1} \# \Sigma^{4 m+1}$ onde:

(a) $S^{2 m} \hookrightarrow M^{4 m+1} \rightarrow S^{2 m+1}$ é o fibrado de esferas associado a qualquer múltiplo de ${ }^{1}$ de $O(2 m+1) \hookrightarrow O(2 m+2) \rightarrow S^{2 m+1}$, fibrado de referenciais de $S^{2 m+1}$

\footnotetext{
${ }^{1} \mathrm{Ou}$ seja, um fibrado cujas funções de transição $\alpha: S^{n-1} \rightarrow G$ são pontualmente múltiplas de $\tau_{2 m}: S^{2 m} \rightarrow$ $O(2 m+1), \tau_{m}^{\mathbb{C}}: S^{2 m} \rightarrow U(m)$ or $\tau_{m}^{\mathbb{H}}: S^{4 m+2} \rightarrow S p(m)$, funções de transição do fibrado de referenciais ortonormais e suas reduções, respectivamente.
} 
(b) $\mathbb{C} P^{m} \hookrightarrow M^{4 m+1} \rightarrow S^{2 m+1}$ é o $\mathbb{C} P^{m}$-fibrado associado a qualquer múltiplo ${ }^{1}$ do fibrado de referências unitários $U(m) \hookrightarrow U(m+1) \rightarrow S^{2 m+1}$

(c) $M^{4 m+1}=\frac{U(m+2)}{S U(2) \times U(m)}$

(v) $\left(M^{8 r+k} \times N^{5-k}\right) \# \Sigma^{8 r+5}$ onde $N^{5-k}$ é qualquer variedade com curvatura de Ricci positiva

(a) $S^{4 r+k-1} \hookrightarrow M^{8 r+k} \rightarrow S^{4 r+1}$ é a k-ésima suspensão do fibrado tangente unitário $S^{4 r-1} \hookrightarrow$ $T^{1} S^{4 r+1} \rightarrow S^{4 r+1}$

(b) para $k=1, \mathbb{H} P^{m} \hookrightarrow M^{8 m+1} \rightarrow S^{4 m+1}$ é o $\mathbb{H} P^{m}$-fibrado associado a qualquer múltiplo ${ }^{1}$ de $S p(m) \hookrightarrow S p(m+1) \rightarrow S^{4 m+1}$

(c) para $k=0, M=\frac{S p(m+2)}{S p(2) \times S p(m)}$

(d) para $k=1, M=M^{8 m+1}$ é como no item (iv).

Teorema 5.5.2. Os seguintes espaços projetivos exóticos $\Sigma \mathcal{P} \mathbb{C}^{n-1}, \Sigma \mathcal{P} \mathbb{H}^{m-1}$ possuem métricas $U(n / 2), S p(m / 2)$-invariantes, respectivamente, com curvatura de Ricci positiva, onde

$$
\begin{aligned}
\Sigma \mathcal{P} \mathbb{C}^{n-1} & :=\Sigma^{2 n-1} / S^{1}, \\
\Sigma \mathcal{P} \mathbb{H}^{m-1} & :=\Sigma^{4 m-1} / S U(2) .
\end{aligned}
$$

Note que a demonstração do Teorema 5.5.1 seguiria como consequência dos Teorema 5.2.1 e 4.1.1. No entanto, fornecemos uma demonstração mais simples e direta. Para tanto, usaremos o seguinte teorema, cuja demonstração é consequência direta do Corolário 5.2.8.

Teorema 5.5.3. Seja $(M, g)$ uma $G$-variedade riemanniana compacta e conexa com $G$ compacto e conexo. Suponha que uma órbita principal segundo a ação por $G$ possua grupo fundamental finito e que $\operatorname{Ric}^{\mathcal{H}}(X)>0, \forall X \neq 0 \in \mathcal{H}_{p}, \forall p \in M$. Então g desenvolve curvatura de Ricci após uma deformação de Cheeger finita.

Seja $M \leftarrow \mathcal{P} \rightarrow M^{\prime}$ um fibrado estrela. O Teorema 4.1.1 garante que dada uma métrica $G$ invariante $g_{M}$ em $M$, existe uma métrica de Kaluza-Klein $G \times G$-invariante $g_{\mathcal{P}}$ em $\mathcal{P}$ e uma única métrica $g_{M^{\prime}}$ em $M^{\prime}$ para qual temos uma submersão riemanniana $\pi^{\prime}:\left(\mathcal{P}, g_{P}\right) \rightarrow\left(M^{\prime}, g_{M^{\prime}}\right)$.

Denote por $\mathcal{L}_{\pi}$ e por $\mathcal{L}_{\pi^{\prime}}$ os levantamentos horizontais por $\pi$ e $\pi^{\prime}$, respectivamente, e por $\mathcal{V}^{\pi}=$ ker $d \pi, \mathcal{V}^{\pi^{\prime}}=\operatorname{ker} d \pi^{\prime}$. Pela aplicação da fórmula (3.3) para $\pi$ e $\pi^{\prime}$, se $X, Y \in\left(\mathcal{V}^{\pi}\right)^{\perp} \cap\left(\mathcal{V}^{\pi^{\prime}}\right)^{\perp}$, então

$$
\begin{aligned}
K_{g_{M}}(d \pi X, d \pi Y) & =K_{g_{\mathcal{P}}}(X, Y)+\frac{3}{4}\left|[X, Y]^{\mathcal{V}^{\pi}}\right|_{g_{\mathcal{P}}}^{2}, \\
K_{g_{M^{\prime}}}\left(d \pi^{\prime} X, d \pi^{\prime} Y\right) & =K_{g_{\mathcal{P}}}(X, Y)+\frac{3}{4}\left|[X, Y]^{\mathcal{V}^{\prime}}\right|_{g_{\mathcal{P}}}^{2} .
\end{aligned}
$$

Denote por $\mathcal{H}, \mathcal{H}^{\prime}$ e $\mathcal{H}^{\prime \prime}$ os complementos ortognais às órbitas de $G$ em $M, M^{\prime}$ e à $G \times G$-órbita em $\mathcal{P}$, respectivamente. Recorde que dado $p \in \mathcal{P}$, então $\left.\mathcal{L}_{p}\right|_{\mathcal{H}_{\pi(p)}}: \mathcal{H}_{\pi(p)} \rightarrow \mathcal{H}_{p}^{\prime \prime}$ e $\left.d \pi^{\prime}\right|_{\mathcal{H}_{p}^{\prime \prime}}: \mathcal{H}_{p}^{\prime \prime} \rightarrow \mathcal{H}_{\pi^{\prime}(p)}^{\prime}$ são isometrias. Ademais, como $\mathcal{H}^{\prime \prime}$ é ortogonal a $\mathcal{V}^{\pi}$ e $\mathcal{V}^{\pi^{\prime}}$, obtemos

$$
K_{g_{M^{\prime}}}\left(d \pi^{\prime} X, d \pi^{\prime} Y\right)=K_{g_{M}}(d \pi X, d \pi Y)+\frac{3}{4}\left(\left|[X, Y]^{\mathcal{V}^{\pi^{\prime}}}\right|_{g_{\mathcal{P}}}^{2}-\left|[X, Y]^{\mathcal{V}^{\pi}}\right|_{g_{\mathcal{P}}}^{2}\right) .
$$

Afirmamos que podemos deformar a métrica $\mathcal{P}$ sem interferir na curvatura $K_{g_{M}}(X, Y)$, mas de forma que o termo $\left|[X, Y]^{\mathcal{V}^{\pi}}\right|_{g_{\mathcal{P}}}^{2}$ seja arbitrariamente pequeno. Precisamente:

Proposição 5.5.4. Seja $M \leftarrow \mathcal{P} \rightarrow M^{\prime}$ um fibrado estrela. Assuma que $\mathcal{P}$ seja compacto. Seja $g_{\mathcal{P}}$ uma métrica $G \times G$-invariante metric em $\mathcal{P}$. Então dado $\epsilon>0$, existe $t>0$ de modo que a métrica $g_{\mathcal{P}_{t}}$, obtido após uma deformação de Cheeger na $\{e\} \times G$-variedade $\mathcal{P}$, satisfaz:

$$
K_{g_{M_{t}^{\prime}}}\left(d \pi^{\prime} X, d \pi^{\prime} Y\right)-K_{g_{M_{t}}}(d \pi X, d \pi Y) \geq-\epsilon|X \wedge Y|_{g_{\mathcal{P}_{t}}}^{2}, \forall X, Y \in \mathcal{H}^{\prime \prime}
$$

onde $g_{M_{t}}, g_{M_{t}^{\prime}}$ são as métricas de submersão induzidas por $g_{\mathcal{P}_{t}}$ em $M$ e $M^{\prime}$, respectivamente. Mais ainda, $g_{M_{t}}=g_{M}$ para todo $t$. 
Demonstração. Note que se $g_{\mathcal{P}}$ é $G \times G$-invariante, então $g_{\mathcal{P}_{t}}$ também o é. De fato, considere $\mathcal{P} \times G$ como uma $G \times G \times G$ variedade cuja ação é definida por

$$
(r, s, q)(p, g)=\left(r p q^{-1}, s g q^{-1}\right)
$$

A ação relativa à deformação de Cheeger (compare com equação (5.1)) é representada pela coordenada $q$. As ações $s, r$ descem para $\mathcal{P}$ como as ações $\bullet$ e $\star$, respectivamente, via projeção no espaço das órbitas $(\mathcal{P} \times G) / G$ segundo ação denotada representada pelos elementos $q \in G$. Logo, considerando a métrica $g_{\mathcal{P}} \times \frac{1}{t} Q$ em $\mathcal{P} \times G$, a métrica por essa induzida em $\mathcal{P}$ é invariante pelas ações • e $\star$. Mais ainda, $\pi:\left(\mathcal{P}, g_{t}\right) \rightarrow\left(M, g_{M}\right)$ é uma submersão riemanniana, uma vez que as deformações de Cheeger só alteram a métrica restrita à distribuição vertical. Para cada $t>0$, tome em $M^{\prime}$ a métrica de submersão riemanniana $g_{M_{t}^{\prime}}$ induzida por $\pi:\left(\mathcal{P}, g_{\mathcal{P}_{t}}\right) \rightarrow\left(M, g_{M_{t}^{\prime}}\right)$ (compare com a equação (3.17)).

Para demonstração a Proposição 5.5.4 lembre-se que o vetor $[X, Y]^{\mathcal{V}^{\pi}}$ só depende das distribuições horizontais e verticais, mas não das suas respectivas métricas. Portanto, para cada métrica $g_{\mathcal{P} t}$ a equação (5.62) é escrita como

$$
K_{g_{M_{t}^{\prime}}}\left(d \pi^{\prime} X, d \pi^{\prime} Y\right)=K_{g_{M}}(d \pi X, d \pi Y)+\frac{3}{4}\left(\left|[X, Y]^{\mathcal{V}^{\pi^{\prime}}}\right|_{g_{\mathcal{P}_{t}}}^{2}-\left|[X, Y]^{\mathcal{V}^{\pi}}\right|_{g_{\mathcal{P}_{t}}}^{2}\right) .
$$

Seja $P$ o tensor de órbitas associado a ação $\bullet$ em $\mathcal{P}$. Dado $p \in \mathcal{P}$, considere a base $g_{\mathcal{P}}$-ortonormal $\left\{v_{1}, \ldots, v_{k}\right\}$ para $\mathcal{V}_{p}^{\pi}$ que consiste nos autovetores associados aos autovalores $\lambda_{1} \leq \ldots \leq \lambda_{k}$. Como a ação principal é livre e $\mathcal{P}$ é uma variedade compacta, existe $\lambda$ tal que $\lambda_{i} \geq \lambda$ para todo $i$ e todo ponto $p$.

Lema 13. Dado $\epsilon^{\prime}>0$, existe $t>0$ tal que para todo $V \in \mathcal{V}^{\pi}$,

$$
|V|_{g_{\mathcal{P}_{t}}}^{2} \leq \epsilon^{\prime}|V|_{g_{P}}^{2}
$$

Demonstração. Dado $V \in \mathcal{V}_{p}^{\pi}$,

$$
g_{\mathcal{P}_{t}}(V, V)=g_{\mathcal{P}}\left((1+t P)^{-1} V, V\right)=\sum_{i, j} g_{\mathcal{P}}\left(g_{P}\left(V, v_{i}\right) v_{i}, g_{P}\left(V, v_{j}\right) v_{j}\right)=\sum_{i} \frac{g_{P}\left(V, v_{i}\right)^{2}}{1+t \lambda_{i}} .
$$

Tomando $t \geq\left(1-\epsilon^{\prime}\right)\left(\lambda \epsilon^{\prime}\right)^{-1}$, obtemos

$$
\sum_{i} \frac{g_{P}\left(V, v_{i}\right)^{2}}{1+t \lambda_{i}} \leq \frac{1}{1+t \lambda}|V|_{g_{\mathcal{P}}}^{2} \leq \frac{1}{1+\frac{1-\epsilon^{\prime}}{\epsilon^{\prime}}}|V|_{g_{\mathcal{P}}}^{2}=\epsilon^{\prime}|V|_{g_{\mathcal{P}}}^{2}
$$

Por fim, lembre-se que o mapa

$$
(X, Y) \mapsto[X, Y]^{\mathcal{V}^{\pi}}
$$

define um tensor antissimétrico $\left(V^{\pi}\right)^{\perp} \times\left(V^{\pi}\right)^{\perp} \rightarrow \mathcal{V}^{\pi}$ (Definição 4). Portanto, sendo $\mathcal{P}$ compacta, existe uma constante $C>0$ tal que

$$
\left|[X, Y]^{\mathcal{V}^{\pi}}\right|_{g_{\mathcal{P}}}^{2} \leq C|X \wedge Y|_{g_{\mathcal{P}}}^{2}
$$

Logo, se tomarmos $t \geq\left(1-\epsilon C^{-1}\right)\left(\lambda \epsilon C^{-1}\right)^{-1}$ o Lema 13 implica que

$$
\left|[X, Y]^{\mathcal{V}^{\pi}}\right|_{g_{\mathcal{P}_{t}}}^{2} \leq \epsilon C^{-1}\left|[X, Y]^{\mathcal{V}^{\pi}}\right|_{g_{\mathcal{P}}}^{2} \leq \epsilon|X \wedge Y|_{g_{\mathcal{P}}}^{2}
$$

Assim,

$$
\begin{aligned}
& K_{g_{M_{t}^{\prime}}}\left(d \pi^{\prime} X, d \pi^{\prime} Y\right)=K_{g_{M}}(d \pi X, d \pi Y)+\frac{3}{4}\left\{\left|[X, Y]^{\mathcal{V}^{\prime}}\right|_{g_{\mathcal{P}_{t}}}^{2}-\left|[X, Y]^{\mathcal{V}^{\pi}}\right|_{g_{\mathcal{P}_{t}}}^{2}\right\} \\
& \geq K_{g_{M}}(d \pi X, d \pi Y)-\epsilon|X \wedge Y|_{g_{P}}^{2} .
\end{aligned}
$$


Trocando $Y$ por elementos $e_{1}, \ldots, e_{r}$, de uma base $g_{\mathcal{P}}$-ortonormal para $\mathcal{H}_{p}^{\prime \prime}$, a Proposição 5.5.4 garante que:

$$
\operatorname{Ric}_{g_{M^{\prime}}}^{\mathcal{H}}\left(d \pi^{\prime} X\right) \geq \operatorname{Ric}_{g_{M}}^{\mathcal{H}}(d \pi X)-\epsilon r\left|d \pi^{\prime} X\right|_{g_{M^{\prime}}}^{2}
$$

Como as variedades $M$ nas construções dos exemplos no Teorema 5.5.1 satisfazem $K_{g_{M}}(d \pi X, d \pi Y)>$ 0 se $X, Y \neq 0$ e portanto $\operatorname{Ric}_{g_{M}}^{\mathcal{H}}(d \pi X)>0$, esse resultado segue como corolário do Teorema 5.5.3 e da Proposição 5.5.4.

Demonstração do Teorema 5.5.2. Considerando o maquinário desenvolvido, apenas notamos que vetores ortogonais às órbitas das ações $U(m)$ e $S p(m)$, respectivamente; levantam para vetores ortogonais às órbitas da ação de $O(n)$ em $\Sigma^{2 n-1}$. Dessa maneira, curvatura de Ricci horizontal positiva em $\Sigma^{2 n-1}$ implica em curvatura de Ricci horizontal positiva em $\Sigma P \mathbb{C}^{2 m-1}$ e $\Sigma P \mathbb{H}^{2 m-1}$. Ademais, as órbitas segundo $U(m)$ e $S p(m)$ possuem grupo fundamental finito, pois os subgrupos de isotropia coincidem com os subgrupos de isotropia da ação bi-axial em $\mathbb{C} P^{2 m-1}$ e $\mathbb{H} P^{2 m-1}$.

\subsection{O conceito de deformações de Cheeger em fibrados com grupo de estrutura compacto}

Nesta seção, extraída do preprint [CS18a], intoduzimos de maneira sistemática uma deformação métrica que estende as deformações de Cheeger para uma classe de fibrados topológicos com grupo de estrutura compacto. Em particular, provamos os Teorema 5.6.1 e 5.6.2, que unificam os principais resultados em [Nas79] e [Poo75]. No Capítulo 6 forneceremos uma prova mais simples e direta para 5.6.2, e o utilizaremos para construir diversos exemplos de fibrados com curvatura de Ricci positiva.

O nosso primeiro resultado pode ser pensado como uma variante do Teorema 0.18 em [FY92].

Teorema 5.6.1. Seja $F \hookrightarrow M \rightarrow B$ um fibrado com grupo de estrutra $G$, fibra $F$ e base $B$ compactos e conexos. Assuma que $M$ seja um fibrado associado a $\pi: \mathcal{P} \rightarrow B$ tal que:

1. As órbitas $G$ em $\mathcal{P}$ tenham curvatura seccional positiva em uma métrica bi-invariante;

2. Existe uma sequência de métricas $g_{n}$ em $\mathcal{P}$ tal que $K_{g_{n}}\left(X^{\mathcal{H}}, Y\right) \geq-\frac{1}{n}$ para qualquer par de vetores unitários $X, Y$ tangentes a $P$ e $\operatorname{diam}\left(g_{n}\right) \leq \frac{1}{n}$;

3. $g_{F}$ possui uma métrica $G$-invariante de curvatura seccional não negativa.

Então $M$ admite uma sequência de métricas riemannianas $\left\{g_{n}^{\prime}\right\}$ tais que $\sec _{g_{n}^{\prime}} \geq-\frac{1}{n}$, $\operatorname{diam}\left(M, g_{n}^{\prime}\right) \leq$ $\frac{1}{n}$.

Uma vez que $\mathcal{P}$ consiste em um fibrado principal a hipótese de órbitas positivamente curvadas em uma métrica $G$-invariante, o grupo de estrutura se restringe a $S^{3}$ ou $S O(3)$.

Teorema 5.6.2. Seja $F \hookrightarrow M \stackrel{\pi}{\rightarrow} B$ um fibrado topológico com base B, fibra $F$ e grupo estrutural $G$ compactos e conexos. Assuma que:

1. B possua uma métrica de curvatura de Ricci positiva,

2. Uma órbita principal da ação de $G$ em $F$ possua grupo fundamental finito,

3. F possua uma métrica G-invariante tal que $\operatorname{Ric}_{F^{r e g} / G} \geq 1$.

Então $M$ admite uma métrica de curvatura de Ricci positiva.

O seguinte corolário garante a existência de diversos exemplos de variedades com métricas de curvatura seccional quase não-negativa e de Ricci positiva, a serem descritos no mesmo capítulo. 
Corolário 5.6.3. Seja $F \hookrightarrow M \stackrel{\pi}{\rightarrow} B$ um fibrado topológico com base $B$, fibra $F$ e grupo estrutural $G$ compactos e conexos. Assuma que:

1. As órbitas de $G$ em $\mathcal{P}$ tenham curvatura seccional positiva em uma métrica bi-invariante;

2. Existe uma sequência de métricas $g_{n}$ em $\mathcal{P}$ tal que $K_{g_{n}}\left(X^{\mathcal{H}}, Y\right) \geq-\frac{1}{n}$ para qualquer par de vetores unitários $X, Y$ tangentes a $P$, $\operatorname{diam}\left(g_{n}\right) \leq \frac{1}{n}$ e $\operatorname{Ric}_{g_{n}}>0$;

3. $g_{F}$ possua uma métrica $G$-invariante de curvatura seccional não negativa e Ricci positiva;

Então $M$ admite uma sequência de métricas $g_{n}^{\prime}$ com curvatura de Ricci positiva e tais que diam $\left(\widetilde{g}_{n}\right) \leq$ $\frac{1}{n}, \sec \left(\widetilde{g}_{n}\right) \geq-\frac{1}{n}$.

Seja $F \hookrightarrow M \stackrel{\pi}{\rightarrow} B$ um fibrado topológico com base $B$, fibra $F$ e grupo estrutural $G$ compactos e conexos. Assuma que a ação efetiva de $G$ em $F$ seja à esquerda. Lembre-se que no desenvolvimento da teoria de deformações de Cheeger consideramos variedades munidas de ações isométricas à esquerda (veja a Seção 5.1). Dito isso, podemos construir um fibrado principal $\mathcal{P}$, obtido a partir das funções de transição de $F$, cuja ação por $G$ é também à esquerda. Mais ainda, adaptando as construções citadas na Seção 3.1, podemos introduzir uma ação efetiva e livre de $G$ em $\mathcal{P} \times F$ (compare com a ação definida em (5.1))

$$
r \cdot(p, f):=(r p, r f), r \in G,(p, f) \in \mathcal{P} \times F,
$$

que tem como espaço de órbitas a variedade $M$.

Dessa maneira, para cada cada par $g$ e $g_{F}$ de métricas $G$-invariantes em $\mathcal{P}$ e $F$ podemos introduzir uma métrica de submersão $h$ em $M$. Precisamente, essa é induzida pela projeção

$$
\bar{\pi}:\left(\mathcal{P} \times F, g \times g_{F}\right) \rightarrow(M, h)
$$

(compare com a Seção 3.1).

Denote por $\mathcal{M}$ a classe de todas as métricas em $M$ obtidas dessa maneira. Afirmamos que $\mathcal{M}$ é relativamente abrangente. De fato, se $G$ age transtivamente em $F$, toda métrica em $M$ que induz métricas $G$-homogêneas nas fibras de $\pi$ é um elemento de $\mathcal{M}$. Chamamos $\mathcal{M}$ de classe das métricas admssiveis para a deformação introduzida a seguir.

Definição 38 (A deformação). Dada $h \in \mathcal{M}$ considere $g \times g_{F}$ a métrica produto em $\mathcal{P} \times F$ para as quais $\bar{\pi}:\left(\mathcal{P} \times F, g \times g_{F}\right) \rightarrow(M, h)$ é uma submersão riemanniana. Definimos $h_{t}$ como a métrica de submersão riemanniana induzida pela métrica $g_{t} \times g_{F}$, onde $g_{t}$ é a deformação de Cheeger no tempo t para a métrica $g$ em $\mathcal{P}$.

Observação. É fundamental ressaltar que aqui, tanto $F$ e $\mathcal{P}$ são $G$-variedades. Então usaremos o mesmo padrão de notações introduzidas na Seção 5.1.

Fixe $(p, f) \in \mathcal{P} \times F$. Qualquer vetor $\bar{X} \in T_{(p, f)}(\mathcal{P} \times F)$ pode se escrito como

$$
\bar{X}=\left(X+X^{\vee}, X_{F}+W^{*}\right)
$$

onde $X$ é ortogonal à órbita de $G$ em $p \in \mathcal{P}, X_{F}$ é ortogonal à órbita de $G$ em $f \in F$ e $V^{\vee}, W^{*}$ são os vetores de ação associados às ações de $G$ em $\mathcal{P}$ e em $F$, respectivamente.

Denote por $P, P_{F}, P_{t}$, respectivamente, os tensores de órbita associados a $g, g_{F}$ e $g_{t}$ (veja por exemplo as equações (5.3), (5.4)). Afirmamos que $\bar{X}$ é $g_{t} \times g_{F}$ ortogonal a $G$-órbita dada pela ação (3.15) se, e somente se, $\exists W \in \mathfrak{m}_{f}$ tal que

$$
\bar{X}=\left(X-\left(P_{t}^{-1} P_{F} W\right)^{\vee}, X_{F}+W^{*}\right)
$$


De fato, um vetor $\left(X+V^{\vee}, X_{F}+W^{*}\right)$ é horizontal na métricas $g_{t} \times g_{f}$ em relação à distribuição vertical dada pela ação (3.15) se, e somente se, para cada $U \in \mathfrak{g}$,

$$
\begin{aligned}
0=g_{t} \times g_{F}\left(\left(X+V^{\vee}, X_{F}+W^{*}\right),\left(U^{\vee}, U^{*}\right)\right) & =g\left(V^{\vee}, U^{\vee}\right)+g_{F}\left(W^{*}, U *\right) \\
& =Q\left(P_{t} V+P_{F} W, U\right) .
\end{aligned}
$$

Como $U$ é arbitrário, $V=-P_{t}^{-1} P_{F} W$, concluindo o afirmado.

Fixado $(p, f) \in \mathcal{P} \times F$, abusaremos notação e denotaremos

$$
d \bar{\pi}_{(p, f)}\left(X, X_{F}+U^{*}\right):=X+X_{F}+U^{*} .
$$

Definimos também os análogos aos tensor de órbita (veja (5.4)) e tensor métrico (veja (5.2)) associados a essa deformação:

$$
\begin{aligned}
& \widetilde{P}_{t}:=P_{F}\left(1+P_{t}^{-1} P_{F}\right)^{-1}=\left(P_{F}^{-1}+P_{t}^{-1}\right)^{-1} \\
& \widetilde{C}_{t}:=-C_{t} P_{t}^{-1} \widetilde{P}_{t}=-P^{-1} \widetilde{P}_{t} .
\end{aligned}
$$

Afirmação 5. Seja $\mathcal{L}_{\bar{\pi}}: T_{\bar{\pi}(p, f)} M \rightarrow T_{(p, f)}(\mathcal{P} \times F)$ o levantamento horizontal associado a $\bar{\pi}$. Então,

$$
\mathcal{L}_{\bar{\pi}}\left(X+X_{F}+U^{*}\right)=\left(X-\left(P_{t}^{-1} \widetilde{P}_{t} U\right)^{\vee}, X_{F}+\left(P_{F}^{-1} \widetilde{P}_{t} U\right)^{*}\right) .
$$

Demonstração. De fato, note que o lado esquerdo da equação (5.71) é da mesma forma da equação (5.67). Portanto, tome $W=P_{F}^{-1} \widetilde{P}_{t} U$, de modo que, $P_{t}^{-1} \widetilde{P}_{t} U=P_{t}^{-1} P_{F} W$. Assim, é suficiente mostrar que

$$
d \bar{\pi}\left(X-\left(P_{t}^{-1} \widetilde{P}_{t} U\right)^{\vee}, X_{F}+\left(P_{F}^{-1} \widetilde{P}_{t} U\right)^{*}\right)=X+X_{F}+U^{*} .
$$

Como ker $d \bar{\pi}=\left\{\left(U^{\vee}, U^{*}\right): U \in \mathfrak{g}\right\}$, a equação (5.68) nos diz que

$$
d \bar{\pi}\left(U^{\vee}, 0\right)=-U^{*}
$$

e assim

$$
d \bar{\pi}\left(X-\left(P_{t}^{-1} \widetilde{P}_{t} U\right)^{\vee}, X_{F}+\left(P_{F}^{-1} \widetilde{P}_{t} U\right) *\right)=X+X_{F}+\left(\left(P_{t}^{-1}+P_{F}^{-1}\right) \widetilde{P}_{t} U\right)^{*}=X+X_{F}+U^{*},
$$

sendo que na última igualdade usamos que $\widetilde{P}_{t}=\left(P_{t}^{-1}+P_{F}^{-1}\right)^{-1}$.

Seguindo de maneira análoga a ideia de Müter em [M̈̈7], calculamos a curvatura seccional de $h_{t}$ para 2-planos parametrizados de forma conveniente. Para tanto, estenda $\widetilde{C}_{t}$ a $T_{\bar{\pi}(p, f)} M$ de maneira natural

$$
\widetilde{C}_{t}\left(X+X_{F}+U^{*}\right):=X+X_{F}+\left(\widetilde{C}_{t} U\right)^{*} .
$$

Denote por $\widetilde{\kappa}_{t}(\widetilde{X}, \widetilde{Y}):=K_{h_{t}}\left(\widetilde{C}_{t}^{-1} \widetilde{X}, \widetilde{C}_{t}^{-1} \widetilde{Y}\right)$.

Teorema 5.6.4 (Curvatura seccional). Seja $h \in \mathcal{M}$ e $g_{t} \times g_{f}$ como na definição 38. Então para quaisquer pares $\widetilde{X}=X+X_{F}+U^{*}, \widetilde{Y}=Y+Y_{F}+V^{*}$ a seguinte fórmula é verdadeira

$$
\widetilde{\kappa}_{t}(\tilde{X}, \tilde{Y})=\kappa_{t}\left(X+U^{\vee}, Y+V^{\vee}\right)+K_{g_{F}}\left(X_{F}-\left(P_{F}^{-1} P U\right)^{*}, Y_{F}-\left(P_{F}^{-1} P V\right)^{*}\right)+\widetilde{z}_{t}(\tilde{X}, \tilde{Y}),
$$

onde $\kappa_{t}$ é como no Teorema 5.1 .1 e $\widetilde{z}_{t}$ é não negativo.

Demonstração. A prova segue inteiramente análoga à prova do Teorema 5.1.1. De fato, como

$$
\mathcal{L}_{\bar{\pi}}\left(\widetilde{C}_{t}^{-1} \tilde{X}\right)=\left(C_{t}^{-1}\left(X+U^{\vee}\right), X_{F}-\left(P_{F}^{-1} P U\right)^{*}\right)
$$


seja $\widetilde{z}_{t}$ três vezes a norma ao quadrado do tensor de O'Neill associado a $\bar{\pi}$ e aplicado a $\mathcal{L}_{\bar{\pi}} \widetilde{C}_{t}^{-1} \widetilde{X}, \bar{\pi} \widetilde{C_{t}}-1 \widetilde{Y}$. Então, pela fórmula de Gray-O'Neill (veja a equação (3.9)),

$$
\begin{aligned}
R_{h_{t}}\left(\widetilde{C}_{t}^{-1} \widetilde{X}, \widetilde{C}_{t}^{-1} \widetilde{Y}, \widetilde{C}_{t}^{-1} \widetilde{Y}, \widetilde{C}_{t}^{-1} \widetilde{X}\right)=K_{g_{t}}\left(C_{t}^{-1}\left(X+U^{\vee}\right), C_{t}^{-1}\left(Y+V^{\vee}\right)\right) \\
+K_{g_{F}}\left(X_{F}-\left(P_{F}^{-1} P U\right)^{*}, Y_{F}-\left(P_{F}^{-1} P V\right)^{*}\right)+\widetilde{z}(\tilde{X}, \widetilde{Y}) \\
=\kappa_{t}\left(X+U^{\vee}, Y+V^{\vee}\right)+K_{g_{F}}\left(X_{F}-\left(P_{F}^{-1} P U\right)^{*}, Y_{F}-\left(P_{F}^{-1} P V\right)^{*}\right)+\widetilde{z} t(\widetilde{X}, \widetilde{Y}) .
\end{aligned}
$$

Embora para nenhum resultado neste texto utilizaremos uma expressão para $\widetilde{z}_{t}$, mostremos que de fato a deformação introduzida na Definição 38 é uma generalização da deformação de Cheeger usual.

Tomando $t=0$ no Teorema 5.6.4 temos

$$
\begin{aligned}
K_{h}(\tilde{X}, \tilde{Y}) & =\widetilde{\kappa}_{0}(\tilde{X}, \widetilde{Y}) \\
& =\kappa_{0}\left(X+U^{\vee}, Y+V^{\vee}\right)+K_{g_{F}}\left(X_{F}-\left(P_{F}^{-1} P U\right)^{*}, Y_{F}-\left(P_{F} P V\right)^{*}\right)+\widetilde{z}_{0}(\widetilde{X}, \widetilde{Y}) .
\end{aligned}
$$

E assim, $\widetilde{z}_{0}$ não é nada mais que o tensor três vezes a norma do tensor de Gray-O'Neill associado à submersão

$$
\bar{\pi}:\left(\mathcal{P} \times F, g \times g_{F}\right) \rightarrow(M, h) .
$$

Mais ainda, $\widetilde{z}_{t}$ é não decrescente. Para ver isso, basta seguir analogamente à demonstração do Teorema 5.1.1. Precisamente, dado $Z \in \mathfrak{g}$, computando a derivada exterior da 1-forma

$$
\widetilde{\omega}_{Z}^{t}\left(X+U^{\vee}, Y+V^{*}\right):=g_{t} \times g\left(\left(X+U^{\vee}, Y+V *\right),\left(Z^{\vee}, Z^{*}\right)\right)
$$

pode-se mostrar que que $d \widetilde{\omega}_{Z}^{t}$ aplicada a vetores horizontais retorna a componente do tensor de GrayO'Neill associado a $\bar{\pi}$ que se expressa na forma $\left(Z^{\vee}, Z^{*}\right)$, e ainda, que $d \widetilde{\omega}_{Z}^{t}\left(\mathcal{L}_{\overline{\bar{\pi}}} \widetilde{C}_{t}^{-1} \widetilde{X}, \mathcal{L}_{\bar{\pi}} \widetilde{C}_{t}^{-1} \widetilde{Y}\right)$ é não decrescente.

Por fim, como $g_{t} \times g\left(\left(Z^{\vee}, Z^{*}\right),\left(Z^{\vee}, Z^{*}\right)\right)$ é não crescente e ainda

$$
\widetilde{z}_{t}(\widetilde{X}, \widetilde{Y})=3 \max _{Z \in \mathfrak{g},|Z|=1} \frac{d \widetilde{\omega}_{Z}^{t}\left(\mathcal{L}_{\overline{\bar{\pi}}} \widetilde{C}_{t}^{-1} \widetilde{X}, \mathcal{L}_{\overline{\bar{\pi}}} \widetilde{C}_{t}^{-1} \widetilde{Y}\right)^{2}}{g\left(\left(Z^{\vee}, Z^{*}\right),\left(Z^{\vee}, Z^{*}\right)\right)}
$$

conclui-se o afirmado.

O próximo Lema demonstra o Teorema 5.6.1.

Lema 14. Sejam $F \hookrightarrow M \rightarrow B$ e $\pi:\left(P, g_{n}\right) \rightarrow B$ como nas hipóteses do Teorema 5.6.1. Então para cada $\epsilon>0$ existem $t_{\epsilon}>0$ e $n_{0} \in \mathbb{N}$ de modo que para cada $t>t_{\epsilon}$ e cada $n>n_{0}$,

$$
\tilde{\kappa}_{t, n}(\tilde{X}, \tilde{Y}) \geq-\epsilon,|\tilde{X}|=|\tilde{Y}|=1 .
$$

Demonstração. Assuma por contradição que existam $\epsilon>0$, sequências $\left\{t_{\epsilon}\right\} \nearrow+\infty, n \nearrow \infty$ e uma sequência de planos $\left\{\widetilde{X}_{t_{\epsilon}, n}, \widetilde{Y}_{t_{\epsilon}, n}\right\}$ tais que $\left|\widetilde{X}_{t_{\epsilon}, n}\right|=\left|\widetilde{Y}_{t_{\epsilon}, n}\right|=1$ satisfazendo

$$
\tilde{\kappa}_{n, t_{\epsilon}}\left(\widetilde{X}_{t_{\epsilon}, n}, \widetilde{Y}_{t_{\epsilon}, n}\right) \leq-\epsilon
$$

Por compacidade, passando a uma subsequência se necessário, extraímos um plano limite $\{\tilde{X}, \tilde{Y}\}$ 
tal que, de acordo com a demonstração do Teorema 5.1.2,

$$
\begin{aligned}
-\epsilon & \geq \lim _{n, t_{\epsilon} \rightarrow \infty}\left\{\kappa_{n, t_{\epsilon}}\left(X_{n, t_{\epsilon}}+U_{n, t_{\epsilon}}^{\vee}, Y_{t_{\epsilon}, n}+V_{n}^{\vee}\right)+K_{g_{F}}\left(\left(X_{F}\right)_{t_{\epsilon}, n}-\left(P_{F}^{-1} P U_{n, t_{\epsilon}}\right)^{*},\left(Y_{F}\right)_{n, t_{\epsilon}}-\left(P_{F}^{-1} P V_{n, t_{\epsilon}}\right)^{*}\right)\right\} \\
& \geq K_{g_{F}}\left(X_{F}, Y_{F}-\left(P_{F}^{-1} P V\right)^{*}\right) \geq 0 .
\end{aligned}
$$

Observação. Mesmo se assumirmos que $K_{F}>, K_{g}>0$ o resultado do Teorema 5.6.1 não pode ser melhorado. Por exemplo, planos tais quais $X=0, U=0, V=0, Y_{F}=0$, isto é, $\widetilde{X}=X_{F}, \tilde{Y}=Y$, satisfazem

$$
\kappa_{0}\left(X, Y+V^{\vee}\right)+K_{g_{F}}\left(X_{F}, Y_{F}-\left(P_{F}^{-1} P V\right)^{*}\right)=0 .
$$

Passemos agora aos preliminares da demonstração do Teorema 5.6.2. Começamos por construir bases apropriadas para o espaço horizontal associado a $\bar{\pi}$ com respeito à métrica $g_{t} \times g_{F}$. Considere uma base $Q$-ortonormal $\left\{v_{k}(0)\right\}$ de $\mathfrak{m}_{f}$ e defina

$$
v_{k}(t)=\tilde{P}_{t}^{-1 / 2} v_{k}(0) .
$$

Lema 15. O conjunto

$$
\left\{\left(-P_{t}^{-1} \tilde{P}_{t} v_{k}(t)^{\vee}, P_{F}^{-1} \tilde{P}_{t} v_{k}(t)^{*}\right)\right\}=\left\{\left(-P_{t}^{-1} \tilde{P}_{t}^{1 / 2} v_{k}(0)^{\vee}, P_{F}^{-1} \tilde{P}_{t}^{1 / 2} v_{k}(0)^{*}\right)\right\}
$$

é $g_{t} \times g_{F}$-ortonormal e $g_{t} \times g_{F}$-ortogonal aos vetores $\left(U^{\vee}, U^{*}\right)$, para cada $U \in \mathfrak{g}$.

Demonstração. Note que os elementos em (5.78) se apresentam na mesma forma da equação (5.67). Então, é suficiente mostrar que o conjunto dado em (5.78) é ortonormal. Calculando diretamente:

$$
\begin{array}{r}
g_{t} \times g_{F}\left(\left(-P_{t}^{-1} \tilde{P}_{t} v_{i}(t)^{\vee}, P_{F}^{-1} \tilde{P}_{t} v_{i}(t)^{*}\right),\left(-P_{t}^{-1} \tilde{P}_{t} v_{j}(t)^{\vee}, P_{F}^{-1} \tilde{P}_{t} v_{j}(t)^{*}\right)\right) \\
=Q\left(\tilde{P}_{t} v_{i}(t), P_{t}^{-1} \tilde{P}_{t} v_{j}(t)\right)+Q\left(\tilde{P}_{t} v_{i}(t), P_{F}^{-1} \tilde{P}_{t} v_{j}(t)\right) \\
=Q\left(\tilde{P}_{t} v_{i}(t),\left(P_{t}^{-1}+P_{F}^{-1}\right) \tilde{P}_{t} v_{j}(t)\right) \\
=Q\left(\tilde{P}_{t} v_{i}(t), v_{j}(t)\right)=Q\left(\tilde{P}_{t}^{1 / 2} v_{i}(0), \tilde{P}_{t}^{-1 / 2} v_{j}(0)\right)=\delta_{i j},
\end{array}
$$

onde usamos que $\left(P_{t}^{-1}+P_{F}^{-1}\right)=\tilde{P}_{t}^{-1}$ e que $\tilde{P}_{t}$ é simétrico.

Sejam $\left\{e_{i}^{B}\right\}$ e $\left\{e_{j}^{F}\right\}$ bases ortonormais para o espaço normal às órbitas em $\mathcal{P}$ e $F$, respectivamente. Completamos o conjunto dado pela Lema 15 a uma base $g_{t} \times g_{F}$-ortonormal para o espaço horizontal associado a $\bar{\pi}$ :

$$
\mathcal{B}_{t}:=\left\{\left(e_{i}^{B}, 0\right),\left(-P_{t}^{-1} \tilde{P}_{t}^{1 / 2} v_{k}(0)^{\vee}, P_{F}^{-1} \tilde{P}_{t}^{1 / 2} v_{k}(0)^{*}\right),\left(0, e_{j}^{F}\right)\right\} .
$$

Denote por $e_{1}, \ldots, e_{n}$ os elementos em $\mathcal{B}_{t}$.

O Teorema 5.6.2 segue dos próximos dois lemas.

Lema 16. Para cada $(p, f) \in \mathcal{P} \times F$ e cada $X+X_{F}+U^{*} \in T_{\bar{\pi}(p, f)} M$

$$
\lim _{t \rightarrow \infty} \operatorname{Ric}_{h_{t}}\left(X+X_{F}+U^{*}\right) \geq \operatorname{Ric}_{g}^{\mathcal{H}}(X)+\operatorname{Ric}_{g_{F}}^{\mathcal{H}}\left(X_{F}\right)+\sum_{k} \frac{1}{4}\left|\left[v_{k}(0), U\right]\right|_{Q}^{2} .
$$


Demonstração. Usando a base $\mathcal{B}_{t}$, definida em (5.79), e o Teorema 5.6.4, temos:

$$
\begin{aligned}
& \operatorname{Ric}_{h_{t}}(\tilde{X})=\sum_{i=1}^{n} \kappa_{t}\left(\tilde{C}_{t} \tilde{X}, \tilde{C}_{t} e_{i}\right) \geq \\
& \sum_{i} \kappa_{t}\left(X-\left(C_{t} P_{t}^{-1} \tilde{P}_{t} U\right)^{\vee}, e_{i}^{B}\right)+\sum_{k} \kappa_{t}\left(C_{t} X-\left(C_{t} P_{t}^{-1} \tilde{P}_{t} U\right)^{\vee},-C_{t} P_{t}^{-1} \tilde{P}_{t}^{1 / 2} v_{k}(0)^{\vee}\right) \\
& \quad+\sum_{j} K_{g_{F}}\left(X_{F}+\left(P_{F}^{-1} \tilde{P}_{t} U\right)^{*}, e_{j}^{F}\right)+\sum_{k} K_{g_{F}}\left(X_{F}+\left(P_{F}^{-1} \tilde{P}_{t} U\right)^{*}, P_{F}^{-1} \tilde{P}_{t}^{1 / 2} v_{k}(0)^{*}\right) .
\end{aligned}
$$

Por outro lado, o tensor $\tilde{P}_{t}$ satisfaz:

$$
\begin{aligned}
\lim _{t \rightarrow \infty} t \tilde{P}_{t} & =1, \\
\lim _{t \rightarrow \infty} P_{t}^{-1} \tilde{P}_{t} & =1 .
\end{aligned}
$$

De fato, $\tilde{P}_{t} \rightarrow 0$ quando $t \rightarrow \infty$. Daí, a equação (5.81) segue dado que $t \tilde{P}_{t}=P_{F}\left(P_{t}+P_{F}\right)^{-1} t P_{t}$, $P_{t} \rightarrow 0$ e que $t P_{t} \rightarrow 1$. Ainda mais, a equação (5.82) segue pois

$$
\lim _{t \rightarrow \infty} P_{t}^{-1} \tilde{P}_{t}=\lim _{t \rightarrow \infty}\left(t P_{t}\right)^{-1} \lim _{t \rightarrow \infty} t \tilde{P}_{t}=1 .
$$

Usando (5.81), notamos que

$$
\begin{array}{r}
\lim _{t \rightarrow \infty}\left\{\sum_{j} K_{g_{F}}\left(X_{F}+\left(P_{F}^{-1} \tilde{P}_{t} U\right)^{*}, e_{j}^{F}\right)+\sum_{k} K_{g_{F}}\left(X_{F}+\left(P_{F}^{-1} \tilde{P}_{t} U\right)^{*}, P_{F}^{-1} \tilde{P}_{t}^{1 / 2} v_{k}(0)^{*}\right)\right\} \\
=\operatorname{Ric}_{g_{F}}^{\mathcal{H}}\left(X_{F}\right) .
\end{array}
$$

Mais ainda, pela equação (5.7) e dado que $C_{t} P_{t}^{-1}=P^{-1}$,

$$
\lim _{t \rightarrow \infty} \sum_{i} \kappa_{t}\left(X-\left(C_{t} P_{t}^{-1} \tilde{P}_{t} U\right)^{\vee}, e_{i}\right) \geq \lim _{t \rightarrow \infty} \operatorname{Ric}_{g}^{\mathcal{H}}\left(X-\left(P^{-1} \tilde{P}_{t} U\right)^{\vee}\right)=\operatorname{Ric}_{g}^{\mathcal{H}}(X) .
$$

Para o termo restante,

$$
\begin{aligned}
\kappa_{t}\left(C_{t} X-\left(C_{t} P_{t}^{-1} \tilde{P}_{t} U\right)^{\vee},-C_{t} P_{t}^{-1} \tilde{P}_{t}^{1 / 2} v_{k}(0)^{\vee}\right) \\
\quad \geq K_{g}\left(X-\left(P^{-1} \tilde{P}_{t} U\right)^{\vee},-P^{-1} \tilde{P}_{t}^{1 / 2} v_{k}(0)^{\vee}\right)+\frac{t^{3}}{4}\left|\left[\tilde{P}_{t} U, \tilde{P}_{t}^{1 / 2} v_{k}(0)\right]\right|_{Q}^{2}
\end{aligned}
$$

Usando as equações (5.81) e (5.82) concluímos que

$$
\lim _{t \rightarrow \infty} \kappa_{t}\left(C_{t} X-\left(C_{t} P_{t}^{-1} \tilde{P}_{t} U\right)^{\vee},-C_{t} P_{t}^{-1} \tilde{P}_{t}^{1 / 2} v_{k}(0)^{\vee}\right) \geq \frac{1}{4}\left|\left[v_{k}(0), U\right]\right|_{Q}^{2} .
$$

O Lema 16 segue juntado as equações (5.83), (5.84) and (5.85).

Lema 17. Seja $F$ uma G-variedade. Assuma que uma órbita principal da ação $G$ e $F$ tenha grupo fundamental finito e que na métrica de distância orbital valha que $\operatorname{Ric}_{F^{r e g} / G} \geq 1$. Então existe uma métrica G-invariante $g_{F}$ em $F$ satisfazendo $\operatorname{Ric}_{g_{F}}^{\mathcal{H}}>0$.

Demonstração. Basta considerar a métrica obtida no Teorema 5.4.1.

Demonstração do Teorema 5.6.2. Seja $g_{F}$ como no Lema 17. Assuma por contradição que, para cada $t>0$, exista um ponto $p_{t} \in M$ e um vetor $h$-unitário $X_{t}+\left(X_{F}\right)_{t}+U_{t}^{*}$ tal que $\operatorname{Ric}_{h_{t}}\left(X_{t}+\right.$ 
$\left.\left(X_{F}\right)_{t}+U_{t}^{*}\right) \leq 0$. Então, considerando $t=n \in \mathbb{N}$, obtemos uma sequência $X_{n}+\left(X_{F}\right)_{n}+U_{n}^{*}$ tal que

$$
\lim _{n \rightarrow \infty} \operatorname{Ric}_{h_{n}}\left(X_{n}+\left(X_{F}\right)_{n}+U_{n}^{*}\right) \leq 0 .
$$

Dado que $\mathcal{P}$ e $F$ são compacto e levantamento horizontal é uma isometria, existe um ponto limite $p$ e um vetor unitário $X+X_{F}+U^{*}$ tal que

$$
\operatorname{Ric}_{g}^{\mathcal{H}}(X)+\frac{1}{4} \sum_{j}\left|\left[v_{j}(0), U\right]\right|_{Q}^{2}+\operatorname{Ric}_{g_{F}}^{\mathcal{H}}\left(X_{F}\right) \leq 0 .
$$

Lembre-se que a soma $\sum_{k} \frac{1}{4}\left|\left[v_{k}(0), U\right]\right|_{Q}^{2}$ é a curvatura de Ricci na métrica $Q$ em $G / G_{f}$, visto como espaço homogêneo normal, na direção de $U$. Como uma órbita principal tem grupo fundamental finito, existe $C>0$ tal que $\frac{1}{4} \sum_{j}\left|\left[v_{j}(0), U\right]\right|_{Q}^{2} \geq C|U|_{Q}^{2}$.

Portanto,

$$
\operatorname{Ric}_{g}^{\mathcal{H}}(X)+\operatorname{Ric}_{g_{F}}^{\mathcal{H}}\left(X_{F}\right)+C|U|_{Q}^{2} \leq 0
$$

o que é uma contradição.

Demonstração do Corolário 5.6.3. Note que as hipóteses permitem concluir, via o Teorema 5.6.1, que $M$ admite uma sequência de métricas $\sec \left(g_{n}^{\prime}\right) \geq-\frac{1}{n}, \operatorname{diam}\left(g_{n}^{\prime}\right) \leq \frac{1}{n}$. Por outro lado, essas são obtidas via uma generalização de deformações de Cheeger, e só modificam a métrica do fibrado principal (é uma deformação de Cheeger nesse). Como deformações de Cheeger não descrescem curvatura, as hipóteses sobre curvatura de Ricci positiva em $\mathcal{P}$ e $F$ são mantidas, e o resultado segue como corolário da demonstração do Teorema 3.1.5 ao notar que ali, as fibras podem ser assumidamente totalmente geodésicas. Assim, a deformação canônica que promove o resultado nada mais é que uma deformação de Cheeger de cada métrica $g_{n}^{\prime}$.

A seguir listamos os resultados em [Nas79] e [Poo75] que são corolários do Teorema 5.6.2.

Corolário 5.6.5 ([Nas79], Theorem 3.5). Seja $\pi: G / H \hookrightarrow E \rightarrow M$ um fibrado topológico com grupo estrutural $G$. Assuma que $M$ seja compacta e admita uma métrica g satisfazendo $\operatorname{Ric}_{g}>0$. Se $G$ é compacto e $G / H$ admite uma métrica tal que $\operatorname{Ric}_{G / H} \geq 1$ (equivalentemente, se $\pi_{1}(G / H)<\infty$ ), então E admite uma métrica de curvatura de Ricci positiva.

Corolário 5.6.6 ([Nas79], Corollary 3.6). Seja $\pi: S^{n} \hookrightarrow E \rightarrow M, n \geq 2$, um fibrado de esferas cuja base $M$ é compacta e admite uma métrica de curvatura de Ricci positiva. Então E admite uma métrica de curvatura de Ricci positiva.

Corolário 5.6.7 ([Nas79], Theorem 3.8). Seja $\pi: F \hookrightarrow E \rightarrow M$ um fibrado com fibra $F$, base $M$ e grupo estrutural $G$ compactos. Assuma ainda que $M$ admita uma métrica $g$ com curvatura de Ricci positiva. Se $\pi_{1}(G)<\infty$, e F admita uma métrica riemanniana $g_{F}$ cuja ação (por isometrias) de $G$ em $F$ é livre e tal que $\operatorname{Ric}_{F / G} \geq 1$, então $E$ admite uma métrica de curvatura de Ricci positiva.

Corolário 5.6.8 ([Poo75], Main Theorem). Sejam $\left(M, g_{M}\right)$ e $\left(F, g_{F}\right)$ variedades riemannianas compactas tais que $g_{M}$ tem curvatura de Ricci positiva e $g_{F}$ tem curvatura seccional positiva. Suponha que $\pi: F M \rightarrow M$ seja um fibrado com fibra $F$ cujo grupo de estrutura $G$ age em $F$ por isometrias. Então FM admite uma métrica de curvatura de Ricci positiva.

\subsection{Uma condição análoga à de Chavez-Derdzinski-Rigas}

Esta seção tem como objetivo generalizar as condições suficientes apresentadas no Teorema 5.1.2 para obteção de métricas de curvatura seccional positiva em $G$-variedades riemannianas. Para tanto, exploraremos o comportamento assintótico das deformações de Cheeger. A fim de motivar trabalhos futuros, começamos com a seguinte discussão.

Seja $(M, g)$ uma $G$-variedade riemanniana. Como apontado em [SSW15], as deformações de Cheeger "renormalizam" a folheação induzida pelas órbitas. Precisamente, no limite $t \rightarrow \infty, g_{t}$ 
se aproxima de uma métrica para quais as órbitas de $G$ em $M$ são totalmente geodésicas. Esse fato aliado à fórmula para curvatura seccional (5.7) levou o Prof. Sperança a formular a seguinte conjectura:

Conjectura 5.7.1. Assuma que a ação de $G$ em $M$ seja livre. Se $M$ possui curvatura seccional positiva, então $M$ admite uma métrica com curvatura seccional não negativa na qual as órbitas por $G$ são totalmente geodésicas.

Essa conjectura, em particular, implica a restrição na dimensão das órbitas em fibrados com grupo de estrutura compacto proposta pela Conjectura de Wilhelm (veja [Spe16c], [GÁG16], [GÁR17]).

Embora a Conjectura 5.7.1 não possa ser demonstrada utilizando somente deformações de Cheeger, podemos proceder da seguinte forma: defina $\tilde{g}_{t}$ partindo das seguintes premissas: o espaço horizontal original, $\mathcal{H}$, é $\tilde{g}_{t}$-ortogonal $\mathcal{V} ;\left.\tilde{g}_{t}\right|_{\mathcal{H}}=\left.g\right|_{\mathcal{H}} ;$ e $\tilde{g}_{t}\left(U^{*}, V^{*}\right)=t^{-1} Q(U, V)$ para todos $U, V \in \mathfrak{g}$. Note que $g_{t}$ se aproxima de $\tilde{g}_{t}$ com ordem $1 / t^{2}$ quando essas são vistas como formas bilineares. Uma tentativa ingênua para a demonstração da Conjectura 5.7.1 consistiria portanto em estabelecer estimativas que levem em conta o fator de aproximação. Entretanto, as curvaturas vertizontais de $g_{t}$ decam com ordem $1 / t^{2}$ e a distância entre $R_{g_{t}}$ e $R_{\tilde{g}_{t}}$ decai na ordem $1 / t$. Esse tipo de normalização segue nas mesmas linhas que em [SSW15].

Estendendo o tensor de órbita $P$ (veja a Definição (5.3)) a toda álgebra de Lie $\mathfrak{g}$ via $P \mathfrak{g}_{p}=$ $0, \forall p \in M$, podemos pensar em $P$ como uma função suave entre $M$ e o espaço dos endomorfismos lineares de $\mathfrak{g}$. Assim, fixada uma bse para $\mathfrak{g}$ para a qual $P=\left(P_{i j}\right), P_{i j} \in C^{\infty}(M ; \mathbb{R})$, a notação Hess $P$ deve ser entendida como a matriz cujas entradas são Hess $P_{i j}$.

Seja $Q$ uma métrica bi-invariante em $G$. A aplicação $\Omega: \mathcal{H}_{p} \times \mathcal{H}_{p} \rightarrow \mathfrak{m}_{p}$ consiste na curvatura da distribuição horizontal $\mathcal{H}$ do fibrado $M^{r e g} \rightarrow M^{r e g} / G$. Especificamente, $\Omega(X, Y)$ é definido como o único elemento em $\mathfrak{m}_{p}$ tal que $\Omega(X, Y)^{*}=-2 A_{X} Y$ (recorde a Definição 4). Analogamente temos

$$
g\left(\Omega_{X}^{*} V^{*}, Y\right)=Q(\Omega(X, Y), V)=-2 g\left(A_{X}^{*} P^{-1} V^{*}, Y\right) .
$$

Em particular, $A^{*} P^{-1} V^{*}=-2 \Omega^{*} V$.

Denote o quociente $M^{r e g} / G$ por $B$ e assuma que esse esteja munido da métrica de submersão induzida no quociente (compare com a equação (3.17)).

Teorema 5.7.2. Assuma que $G$ seja compacto e que para cada $p \in M^{r e g}, G / G_{p}$ possua curvatura seccional positiva na métrica de espaço homogêneo normal. Então existe $t>0$ tal que $\sec _{g_{t}}>0$ se, e somente se, existir $k>0$ tal que

$$
\begin{array}{r}
\left(K_{B}(X, Y)-k|X \wedge Y|_{g}^{2}\right)\left(\frac{1}{2} Q\left(\operatorname{Hess} P^{-1}(X) V, V\right)+\frac{1}{4}\left|\Omega_{X}^{*} V\right|_{g}^{2}-k|X|_{g}^{2} Q\left(P^{-1} V, V\right)\right) \\
\geq \frac{1}{4} Q\left(\left(\nabla_{X} \Omega\right)_{X} Y, V\right)^{2}
\end{array}
$$

para todos $X, Y \in \mathcal{H}_{p}, V \in \mathfrak{m}_{p}, p \in M^{\text {reg }}$.

Demonstração. Utilizando a equação (5.7) e o fato que $\kappa$ é contínua em $M^{r e g}$, observamos que existe $t>0$ tal que $\sec _{g_{t}}>0$ se, e somente se, existir $k>0$ tal que $\lim _{t \rightarrow \infty} \kappa_{t}\left(X, Y+V^{*}\right) \geq$ $k\left|X+\left(Y+V^{*}\right)\right|_{g}^{2}$. Mais ainda, é suficiente mostrar que, para qualquer família de isomorfismos lineares $L_{t}$,

$$
\lim _{t \rightarrow \infty} \kappa_{t}\left(L_{t} X, L_{t}\left(Y+V^{*}\right)\right) \geq k\left|X+\left(Y+V^{*}\right)\right|_{g}^{2}
$$

Seja $p \in M^{\text {reg }}$ e considere o plano ortonormal $\{\bar{X}, \bar{Y}\} \subset \operatorname{Gr}_{2}\left(\mathcal{T}_{p} M\right)$. Lembre-se que o plano $\left\{C_{t}^{-\frac{1}{2}} \bar{X}, C_{t}^{-\frac{1}{2}} \bar{Y}\right\} \subset \operatorname{Gr}_{2}\left(\mathcal{T}_{p} M\right)$ é $g_{t}$-ortonormal e portanto, podemos computar

$$
R_{g_{t}}\left(C_{t}^{-\frac{1}{2}} X, C_{t}^{-\frac{1}{2}}\left(Y+V^{*}\right), C_{t}^{-\frac{1}{2}}\left(Y+V^{*}\right), C_{t}^{-\frac{1}{2}} X\right)=\kappa_{t}\left(C_{t}^{\frac{1}{2}} X, C_{t}^{\frac{1}{2}}\left(Y+V^{*}\right)\right) .
$$

Note que o lado direito decai com $t^{-2}$. Assim, considere a seguinte família de isomorfismos lineares

$$
L_{t}\left(Y+V^{*}\right)=Y+t^{\frac{1}{2}} C_{t}^{\frac{1}{2}} P^{-\frac{1}{2}} V^{*} .
$$


O termo $t^{\frac{1}{2}}$ em $L_{t}$ corrige o decaimento de $C_{t}^{\frac{1}{2}}$. Mais ainda, o termo $P^{-\frac{1}{2}}$ funciona como uma renormalização que independe de $t$ para a folheação induzida pelas órbitas regulares. De fato, note que $g\left(P^{-\frac{1}{2}} V^{*}, P^{-\frac{1}{2}} U^{*}\right)=Q(V, U)$. Ainda mais, um cálculo direto mostra que $L_{t} \rightarrow P^{-1}$. Levando isso em conta, separamos a expressão para curvatura

$$
\begin{aligned}
\lim _{t \rightarrow \infty} \kappa_{t}(X, Y & \left.+t^{\frac{1}{2}} C_{t}^{\frac{1}{2}} P^{-\frac{1}{2}} V^{*}\right)= \\
& =\kappa_{0}(X, Y)+2 R_{g}\left(X, Y, P^{-1} V^{*}, X\right)+\kappa_{0}\left(X, P^{-1} V^{*}\right)+z_{\infty}\left(X, Y+P^{-1} V^{*}\right) .
\end{aligned}
$$

A equação (5.24) diz que

$$
z_{\infty}\left(X, Y+V^{*}\right)=3\left|A_{X} Y+S_{X} V^{*}\right|_{g}^{2}
$$

Seguindo o quadractic trick podemos pensar na equação (5.90) como sendo quadrática em $V^{*}$. Assim, recordando as equações (3.11) e $\kappa_{0}(X, Y)=K_{B}(X, Y)-3\left|A_{X} Y\right|^{2}$, concluímos que existe $k>0$ tal que

$$
\lim _{t \rightarrow \infty} \kappa_{t}\left(X, Y+t^{\frac{1}{2}} C_{t}^{\frac{1}{2}} P^{-\frac{1}{2}} V^{*}\right) \geq k\left|X \wedge\left(Y+V^{*}\right)\right|_{g}^{2}
$$

se, e somente, se, existir $k>0$ tal que

$$
\begin{aligned}
\left(K_{B}(X, Y)-k|X \wedge Y|_{g}^{2}\right)\left(\kappa_{0}\left(X, P^{-1} V^{*}\right)-\right. & \left.3\left|S_{X} P^{-1} V^{*}\right|_{g}^{2}-k|X|_{g}^{2} Q\left(P^{-1} V, V\right)\right) \\
& \geq \frac{1}{4}\left(R_{g}\left(X, Y, P^{-1} V^{*}, X\right)+3 g\left(S_{X} P^{-1} V^{*}, A_{X} Y\right)\right)^{2} .
\end{aligned}
$$

Seja $\gamma(s)$ a geodésica definida por $X$. Como $V^{*}(s)$ é um campo de Killing ao longo de $\gamma(s)$, concluímos que $V^{*}$ é um Campo de Jacobi de Holonomia ao longo de $\gamma$ (veja [GW09, Definition 1.4.3]), i.e., $\nabla_{X}^{\mathcal{V}} V^{*}=-S_{X} V^{*}$. Ademais, para qualquer $W \in \mathfrak{g}$, o termo

$$
g\left(W^{*}, P_{s}^{-1} V^{*}\right)=Q\left(P_{s} W, P_{s}^{-1} V\right)=Q(W, V)
$$

não depende de $s$.

Para obter a expressão

$$
\kappa_{0}\left(X, P^{-1} V^{*}\right)-3\left|S_{X} P^{-1} V^{*}\right|^{2}=\frac{1}{2} Q\left(\operatorname{Hess} P^{-1}(X) V, V\right)+\frac{1}{4}\left|\Omega_{X}^{*} V\right|_{g}^{2},
$$

notamos que

$$
\nabla_{X}^{\mathcal{V}}\left(P_{s}^{-1} V\right)^{*}=S_{X}\left(P_{s}^{-1} V\right)^{*}
$$

De fato,

$$
\begin{aligned}
g\left(\nabla_{X}^{\mathcal{V}}\left(P_{s}^{-1} V\right)^{*}, W^{*}\right) & =X g\left(\left(P_{s}^{-1} V\right)^{*}, W^{*}\right)-g\left(\left(P_{s}^{-1} V\right)^{*}, \nabla_{X}^{\mathcal{V}} W^{*}\right) \\
& =-g\left(\left(P_{s}^{-1} V\right)^{*}, \nabla_{X}^{\mathcal{V}} W^{*}\right) \\
& =g\left(\left(P_{s}^{-1} V\right)^{*},\left(S_{X} W^{*}\right)\right) \\
& =g\left(S_{X}\left(P_{s}^{-1} V\right)^{*}, W^{*}\right) .
\end{aligned}
$$

Assim, a equação (5.92) segue de um cálculo direto utilizando a equação (3.11).

O termo $R_{g}\left(X, Y, P^{-1} V^{*}, X\right)+3 g\left(S_{X}\left(P^{-1} V\right)^{*}, A_{X} Y\right)$ aparece em [Spe16c] e pode ser identificado com $-Q\left(\frac{d}{d s} \Omega(X, Y), V\right)$. De fato, como

$$
R_{g}\left(X, Y,\left(P_{s}^{-1} V\right)^{*}, X\right)=g\left(\left(\nabla_{X} A\right)_{X} Y, P_{s}^{-1} V^{*}\right)-2 g\left(S_{X}\left(P_{s}^{-1} V\right)^{*}, A_{X} Y\right),
$$


usando a tanto a definição de $\Omega$ quanto a equação (5.93) obtemos

$$
\begin{aligned}
R_{g}\left(X, Y,\left(P^{-1} V\right)^{*}, X\right)+3 g\left(S_{X}\left(P^{-1} V\right)^{*}, A_{X} Y\right) & =g\left(\left(\nabla_{X} A\right)_{X} Y,\left(P^{-1} V\right)^{*}\right)+g\left(A_{X}^{*} S_{X}\left(P^{-1} V\right)^{*}, Y\right) \\
& =g\left(\nabla_{X}\left(A_{X} Y\right),\left(P_{s}^{-1} V\right)^{*}\right)+g\left(A_{X}^{*}\left(S_{X}\left(P_{s}^{-1} V\right)^{*}\right), Y\right) \\
& =2 g\left(A_{X}^{*}\left(S_{X}\left(P_{s}^{-1} V\right)^{*}\right), Y\right) \\
& =-g\left(S_{X}\left(P_{s}^{-1} V\right)^{*}, \Omega(X, Y)^{*}\right) \\
& =-g\left(\left(P_{s}^{-1} V\right)^{*}, \nabla_{X}^{\mathcal{V}} \Omega(X, Y)^{*}\right) \\
& =-Q\left(V, \nabla_{X}^{\mathcal{V}} \Omega(X, Y)\right) .
\end{aligned}
$$




\section{Capítulo 6}

\section{Um zoológico de variedades com curvatura seccional quase não-negativa e de Ricci positiva}

\subsection{Prelúdio}

Começamos por reenunciar e apresentar uma demonstração muito simplificada para o Teorema 5.6.2, demonstrado no Capítulo 5.

Teorema 6.1.1. Seja $F \hookrightarrow M \stackrel{\pi}{\rightarrow} B$ um fibrado com $M, F, B$ e grupo estrutural $G$ compactos. Assuma que:

1. B tenha uma métrica de curvatura de Ricci positiva,

2. Uma órbita principal de $G$ em $F$ tem grupo fundamental finito,

3. F possua uma métrica $G$-invariante tal que $\operatorname{Ric}_{F^{r e g} / G} \geq 1$.

Então $M$ possui uma métrica de curvatura de Ricci positiva.

Demonstração do Teorema 6.1.1. Pela hipótese que $F^{r e g} / G$ admite uma métrica de curvatura de Ricci positiva e pelo fato que uma órbita principal de $F$ por $G$ tem grupo fundamental finito, como consequência Teorema $\mathrm{C}$ ou 5.4.1, $F$ admite uma métrica $G$-invariante com curvatura de Ricci positiva. Agora estamos nas hipóteses do Teorema 3.1.5, que implica que $M$ admite uma métrica de curvatura de Ricci positiva.

Observação. Destacamos que no caso de fibrados principais, a métrica com curvatura de Ricci positiva obtida no Teorema 6.1 .1 pode ser assumida $G$-invariante. O candidato gostaria de agradecer ao Prof. W. Tuschmann por levantar essa questão.

Este capítulo tem como um dos objetivos construir diversos novos exemplos de variedades com curvatura de Ricci positiva e, sempre que possível, exemplos com métricas de curvatura seccional quase não negativa e Ricci positiva, simultanemante. Uma métrica de curvatura seccional quase não negativa é como no Lema 14.

A ideia central consiste em empregar o Teorema 6.1.1, onde o espaço total será realizado como fibrado associado aos fibrados principais definidos por diagramas estrela (recorde o Capítulo 4). Mais ainda, utilizaremos os exemplos de Shrinking Ricci Solitons (SRS) $(M, g, f)$ obtidos por DancerWang (Teoremas 3.3.4, 3.3.5) como base em fibrados topológicos, e cujo espaço total é um produto vertical $M \times_{e^{2 f}} F$, e cuja fibra $F$ consiste em todo e qualquer exemplo de variedade com curvatura de Ricci positiva que satisfaça as hipóteses do Teorema 5.2.1. Por exemplo, todas as variedades construídas no Teorema 4.1.1. 


\subsection{Construção dos exemplos}

Sejam $B, F$ variedades satisfazendo as hipóteses do Teorema 5.2.1. Dado um $G$-fibrado principal,

$$
\pi: G \hookrightarrow P \rightarrow B
$$

o Teorema 6.1.1 garante a existência de uma métrica de curvatura de Ricci positiva em fibrados associados pr : $F \hookrightarrow P \times_{G} F \rightarrow B$. Nesta subseção, combinamos essa ideia com as construções apresentadas no Capítulo 4, para construrir novos exemplos de fibrados com curvatura de Ricci positiva.

Exemplo. Considere P, $M, M^{\prime}, G$ como no diagrama (4.1) e assuma que $M$ satisfaça as hipóteses do Teorema 5.2.1. Então, de acordo com o Teorema 4.2.3, podemos assumir que $M^{\prime}$ também satisfaz tais hipóteses. Portanto, o Teorema 6.1.1 garante a existência de uma métrica de curvatura de Ricci positiva nos seguintes fibrados

1. O fibrado associado $M \hookrightarrow P \times_{G} M \rightarrow M a \pi: P \rightarrow M$,

2. O fibrado associado $M^{\prime} \hookrightarrow P \times_{G} M^{\prime} \rightarrow M a \pi: P \rightarrow M$,

3. O fibrado associado $M \hookrightarrow P \times_{G} M^{\prime} \rightarrow M^{\prime}$ a $\pi^{\prime}: P \rightarrow M^{\prime}$,

4. O fibrado associado $M^{\prime} \hookrightarrow P \times_{G} M^{\prime} \rightarrow M^{\prime}$ a $\pi^{\prime}: P \rightarrow M^{\prime}$.

Note que $M^{\prime}$ pode ser tomada como qualquer variedade construída no Teorema 4.1.1, e $M$ como sua contraparte, ou seja, a outra variedade base num diagrama como (4.1).

Exemplo. Daremos mais detalhes sobre as possiveis construções mencionadas no item anterior. Considere uma construção via um diagrama estrela (diagrama (4.1)) com $G=S^{3}, P=S p(2)$, $M=S^{7}, M^{\prime}=\Sigma^{7}$, onde $\Sigma^{7}$ é a esfera exótica de Gromoll-Meyer. Seja B uma variedade satisfazendo as hipóteses do Teorema 5.2.1. Assuma que exista o seguinte fibrado principal $\mathrm{pr}: S^{3} \hookrightarrow S p(2) \rightarrow B$.

(i) Seja $S p(2) \hookrightarrow S p(2) \times{ }_{S^{3}} S p(2) \rightarrow B$ o fibrado associado a pr: $S^{3} \hookrightarrow S p(2) \rightarrow B$. Pelo Teorema 6.1.1, esse fibrado amite uma métrica com curvatura de Ricci positiva. Mais ainda, como $S p(2)$ possui uma métrica $\bullet$ (resp. *)-invariante de curvatura seccional não negativa e de Ricci positiva, o Corolário 5.6.3 garante que esse fibrado admite uma métrica de curvatura seccional quase não negativa e Ricci positiva.

(ii) Note que podemos assumir, por exemplo, que $B$ seja $S^{7}$ ou $\Sigma^{7}$. Assuma que esse seja o caso. Então, quando considerarmos $B=S^{7}$, pensaremos nessa como uma $S^{3}$-variedade munida da ação $\bullet$. Analogamente, se $B=\Sigma^{7}$, pensaremos nessa como uma $S^{3}$-variedade munida da ação *. Em ambos os casos, a $S^{3}$-variedade $B$ pode ser assosciada como fibra para os fibrados principais $\pi: S p(2) \rightarrow S^{7}$ e $\pi^{\prime}: S p(2) \rightarrow \Sigma^{7}$, descritos pelo diagrama (4.6), produzindo novos exemplos de fibrados com curvatura seccional quase não-negativa e de Ricci positiva. Explicitamente, os seguintes fibrados admitem uma métrica com tais propriedades;

(a) O fibrado associado $S^{7} \hookrightarrow S p(2) \times_{S^{3}} S^{7} \rightarrow \Sigma^{7}$ a $\pi^{\prime}: S p(2) \rightarrow \Sigma^{7}$,

(b) O fibrado associado $\Sigma^{7} \hookrightarrow S p(2) \times{ }_{S^{3}} \Sigma^{7} \rightarrow \Sigma^{7}$ a $\pi^{\prime}: S p(2) \rightarrow \Sigma^{7}$,

(c) O fibrado associado $\Sigma^{7} \hookrightarrow S p(2) \times_{S^{3}} \Sigma^{7} \rightarrow S^{7}$ a $\pi: S p(2) \rightarrow S^{7}$,

(d) O fibrado associado $S^{7} \hookrightarrow S p(2) \times{ }_{S^{3}} S^{7} \rightarrow S^{7}$ a $\pi: S p(2) \rightarrow S^{7}$.

(iii) Segundo os itens (a), (b), podemos construir a seguinte escadaria de fibrados com curvatura de 
Ricci positiva:

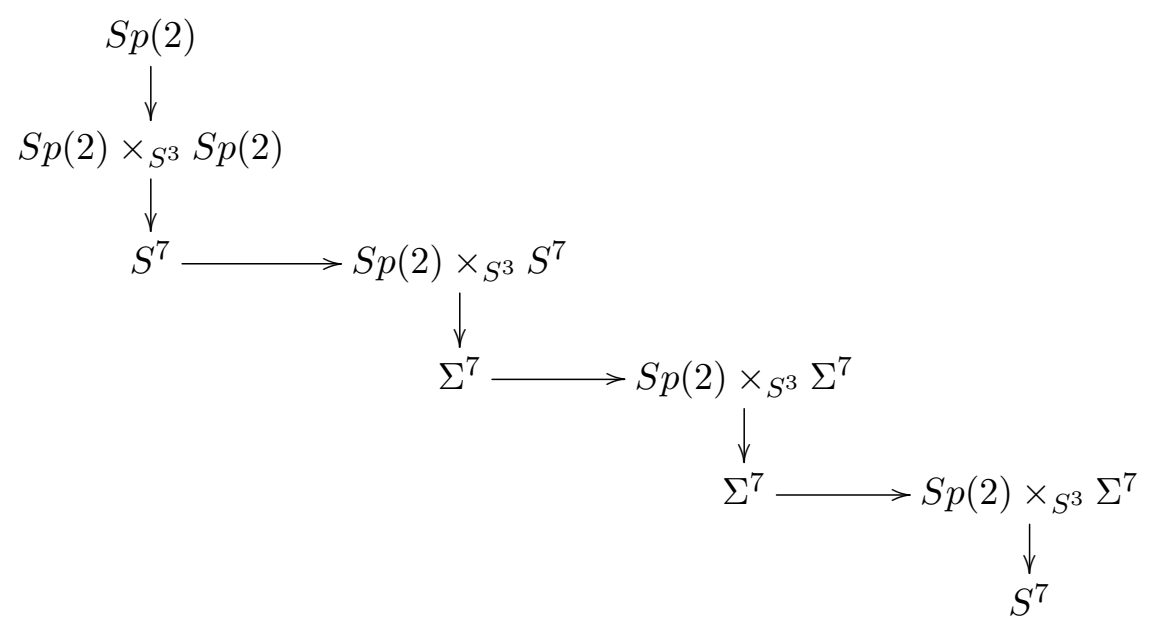

Vale ressaltar que o fibrado em cada etapa pode ser empilhado acima daquele na próxima etapa (inferior), resultando em um cluster de fibrados. Por exemplo ${ }^{1}$ :

(a) $S p(2){ }_{\star} \times_{\star}\left(S p(2) \bullet \times_{\bullet} S p(2)\right) \rightarrow \Sigma^{7}$ é um fibrado cuja fibra típica é o espaço total do fibrado $S p(2) \bullet{ }_{\bullet} S p(2) \rightarrow S^{7}$ (aqui a projeção deve ser vista como projeção no primeiro $S p(2)$ fator em $\left.S p(2) / \star=\Sigma^{7}\right)$;

(b) $S p(2)_{\star} \times \bullet\left(S p(2)_{\star} \times_{\star}\left(S p(2) \bullet \times_{\bullet} S p(2)\right)\right) \rightarrow \Sigma^{7}$ é um fibrado cuja fibra típica é o espaço total do fibrado no item $(a)$;

(c) $S p(2)_{\star} \times \bullet\left(S p(2)_{\star} \times \bullet\left(S p(2){ }_{\star} \times_{\star}\left(S p(2) \bullet \times_{\bullet} S p(2)\right)\right)\right) \Sigma^{7}$ é o fibrado cuja fibra típica é o espaço total do fibrado no item (b);

(d) o fibrado representado por toda escadaria é

$$
S p(2) \bullet \times_{\bullet}\left(S p(2)_{\star} \times \bullet\left(S p(2)_{\star} \times \bullet\left(S p(2){ }_{\star} \times_{\star}\left(S p(2) \bullet \times_{\bullet} S p(2)\right)\right)\right)\right) \rightarrow S^{7} .
$$

De maneira mais geral, qualquer combinação $*_{1}, \ldots, *_{2 k}, *_{i} \in\{\bullet, \star\}$, em

$$
S p(2)_{*_{1}} \times_{*_{2}} S p(2)_{*_{3}} \times_{*_{4}} \cdots *_{*_{2 k-1}} \times_{*_{2 k}} S p(2) \rightarrow S p(2) / *_{1}
$$

pode ser realizada como uma escadaria de fibrados sobre $S p(2) / *_{1}$, desde que $*_{2 m} \neq *_{2 m+1}$. Aplicando recursivamente o Teorema 6.1.1, concluí-se que (6.2) admite uma métrica com curvatura de Ricci positiva. De fato, assumindo que $P \rightarrow M$ seja um $G$-fibrado principal com uma $H$-ação adicional que comute com a ação por $G$, então $P$ admite uma 1-forma de conexão $H$-invariante. Mais ainda, a variação canônica de uma métrica de conexão $H$ invariante é também $H$-invariante se o espaço horizontal seja obtido por uma forma de conexão $H$-invariante.

Exemplo. Uma construção similar ao exemplo precedente funciona no caso em que $G=S^{3}, P=$ $f^{*}(S p(2)), M=S^{8}, M^{\prime}=\Sigma^{8}$, onde $M^{\prime}$ é a única esfera exótica de dimensão 8 e $f$ é o mapa

$$
\begin{gathered}
f: S^{8} \rightarrow S^{7} \\
f(\lambda, x, w):=\frac{1}{\sqrt{\lambda+|x|^{4}+|w|^{2}}}(\lambda+x i \bar{x}, w),
\end{gathered}
$$

quando $S^{8}$ é vista como a esfera unitária em $\mathbb{R} \times \mathbb{H}^{2}$. Para detalhes sobre a realização de $\Sigma^{8}$ via diagrama estrela, veja [Spe16b].

\footnotetext{
${ }^{1}$ usamos a notação $S p(2) *_{1} \times_{*_{2}} S p(2)$ com o intuito de justificar que $S^{3}$-ação estamos considerando. Precisamente, $S p(2) *_{1} \times_{*_{2}} S p(2)$ denota o quociente de $S p(2) \times S p(2)$ pela $S^{3}$-ação $q(A, B)=\left(q *_{1} A, q *_{2} B\right)$
} 
Ainda considerando um diagrama como (4.1), seja $H$ um subgrupo de Lie de $G$ tal que $\pi_{1}(G / H)<$ $\infty$. Restringindo as ações $\bullet$ e a $H$, construímos o seguinte diagrama estrela:

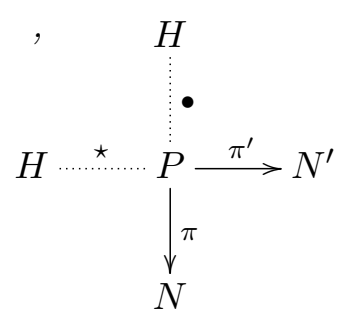

onde $N^{\prime}=P / \star, N=P / \bullet$, ou seja, são os espaços de órbitas em (6.4).

Primeiramente, note que $N$ e $N^{\prime}$ admitem uma métrica de curvatura de Ricci positiva se $M$ ou $M^{\prime}$ satisfizer as hipóteses do Teorema 5.2.1. De fato, lembre-se que tanto para $\bullet$ como para $\star$ tem-se fibrações

$$
G / H \hookrightarrow P / H \rightarrow P / G
$$

Como $P / G$ é identificada com $M$ ou com $M^{\prime}$, dependendo da ação considerada (• ou $\star$ ) em $P$, se $M$ ou $M^{\prime}$ satisfazem as hipóteses do Teorema 5.2.1, então $P / H$ admite uma métrica de curvatura de Ricci positiva.

Assim, analogamente aos itens anteriores, podemos construrir 6 novos fibrados com curvatura de Ricci positiva. De fato, sendo $\pi$ como no diagrama (6.4), podemos construrir fibrados associados a $\pi$ cujas fibras são $M, M^{\prime}, P$.

Exemplo. De maneira geral, desde que $*_{2 m} \neq *_{2 m+1}, m=1, \ldots, k-1$, as construções nos exemplos prescedentes se estendem para qualquer variedade $P$ satisfazendo (1.1), trocando simplesmente $S p(2)$ por P em (6.2):

$$
P_{*_{1}} \times_{*_{2}} P_{*_{3}} \times_{*_{4}} \cdots *_{*_{2 k-1}} \times_{*_{2 k}} P \rightarrow P / *_{1}
$$

Pode-se ainda generalizar tal construção para qualquer família $P_{0}, \ldots, P_{k}$, onde $P_{i}$ está equipado com uma $G_{i} \times H_{i}$-ação, originando um cluster de fibrados como em (6.1), desde que as $G_{i^{-}} H_{i}$-ações sejam livres e $H_{i}=G_{i+1}$ (a menos de isomorfismo):

$$
P_{0 H_{0}} \times_{G_{1}} P_{1 H_{1}} \times_{G_{2}} \cdots H_{k-1} \times_{G_{k}} P_{k} \rightarrow P_{0} / H_{0} .
$$

Por exemplo, $P_{0}, \ldots, P_{k}$ podem ser tomados como quaisuqer combinações dos fibrados construídos em [CS18b] via construção $\star$.

Aproveitamos para destacar dois pontos interessantes sobre a construção iterada apresentada em (6.6),(6.7). Essas contornam dois problemas. Por um lado, para empilhar um fibrado sobre a fibra típica de outro, geralmente procedemos levantando funções de transição. Precisamente, dados dois fibrados $F \hookrightarrow P \rightarrow M$ e $E \rightarrow F$, uma maneira de construrir um novo fibrado sobre $M$ cuja fibra é $E$ consiste em levantar as funções de transição; que tomam valores em $\operatorname{Diff}(F) ;$ para $\operatorname{Diff}(E)$. Com esse procedimento tal passo é evitado.

Um segundo problema que poderia ocorrer ao ignorar a construção iterada, reside no fato de que os quocientes $P_{0} H_{0} \times{ }_{G_{1}} P_{1 H_{1}} \times_{G_{2}} \cdots H_{H_{-1}} \times_{G_{k}} P_{k}$ não precisariam admitir métricas de curvatura de Ricci positiva, como destacado em [PW14]. Entretanto, esses são espaços totais de fibrados com fibra e base de curvatura de Ricci positiva, admitindo portanto uma métrica com curvatura de Ricci positiva.

Exemplo. Seja F qualquer variedade com curvatura de Ricci positiva construída com os métodos desta tese, por exemplo, seja $F$ qualquer variedade dada pelo Teorema 4.1.1. Então, como consequência do Teorema 3.3.6, as seguintes variedades admitem uma métrica de curvatura de Ricci positiva:

1. $\widehat{M}_{1}:=M \times_{e^{2 f}} F$, onde $(M, g, f)$ é um SRS não trivial tal que g é variedade de Kähler e fano, construído no espaço total de um $\mathbb{C P}^{1}$-fibrado sobre $\mathbb{C P}^{2} \times \mathbb{C P}^{2}$, 
2. $\widehat{M}_{2}:=M \times_{e^{2 f}} F$, onde $(M, g, f)$ é um SRS não trivial tal que $g$ é variedade de Kähler e fano, construído no espaço total de um $\mathbb{C P}^{1}$-fibrado sobre um produto de duas cópias de $\mathcal{F}(3,3)$, onde $\mathcal{F}(3,3)$ é a variedade irrational Clemens-Griffiths three-fold,

3. Mais ainda, podemos proceder indefinitamente ao considerar $\widehat{M}_{i+1}:=M \times_{e^{2 f}} \widehat{M}_{i}, i \in\{1,2, \ldots\}$, obtendo novas variedades com curvatura de Ricci positiva. Aqui, $(M, g, f)$ pode ser tomada como qualquer exemplo de SRS dos itens anteriores. 


\section{Capítulo 7}

\section{Sobre funções de curvatura escalar admssíveis em fibrados com grupo de estrutura compacto}

O problema sobre quais funções podem ser funções de curvatura escalar para alguma métrica riemanniana em uma variedade fechada e conexa foi completamente resolvido no trabalho seminal de Kazdan e Warner (e.g. [KW75b, KW75c]). Por exemplo, se uma variedade fechada e conexa $M$ admite uma métrica de curvatura escalar positiva constante, então qualquer função suave $f: M \rightarrow \mathbb{R}$ é a curvatura escalar para alguma métrica em $M$. Mais ainda, uma vez que existem obstruções topológicas para curvatura escalar estritamente positiva, negativa, ou zero, seus resultados são ótimos.

A abordagem nesses trabalhos consiste essencialmente na busca de métricas por meio de mudanças conformes de uma métrica pré-fixada. Neste capítulo nós lidamos com uma extensão natural desse problema. Precisamente, consideramos classes de fibrados com hipóteses bastante gerais e procuramos determinar quais funções são admssíveis como curvatura escalar para métricas de submersão riemanniana no espaço total desses fibrados. Como por um lado métricas de submersão definem uma isometria entre a distribuição horizontal no espaço total e o espaço tangente à base, e por outro, a fórmula de submersão de Gray-O'Neill (3.3) implica que a curvatura escalar da base é sempre crescente, naturalmente pode-se esperar outras obstruções sobre as funções admssíveis. Nossos resultados reforçam esses fatos, uma vez que as condições suficientes para uma função $f$ ser uma função de curvatura escalar em um fibrado depende, no caso em que as fibras tem curvatura escalar não positiva em um ponto, da geometria da base (Teoremas 7.2.1, 7.2.2). Quando essa admite uma métrica de curvatura de Ricci positiva, então as exigências sobre $f$ ficam completamente determinadas pela geometria da fibra (Teorema 7.1.1).

Seja $\pi:\left(F, g_{F}\right) \hookrightarrow(M, g) \rightarrow(B, h)$ uma submersão riemanniana com $M$ fechada e conexa e $F$ compacta. Os resultados obtidos são provados considerando produtos verticais gerais de $g$. Precisamente, ao invés de procurar por mudanças conformes, analisamos somente deformações nas direções verticais (tangentes à folheação) da métrica $g$. Assim, naturalmente a métrica que eventualmente realiza determina função de curvatura escalar será uma métrica de submersão riemanniana.

\subsection{Fibrados Topológicos com fibras com curvatura escalar positiva}

Nesta seção resolveremos o problema de Kazdan-Warner no contexto de fibrados (principais e gerais) que admitem métricas de curvatura de Ricci positiva. Mais ainda, no caso de fibrados principais, as métricas que realizam as funções prescritas como funções de curvatura escalar são $G$-invariantes. Precisamente, provamos que:

Teorema 7.1.1. Seja $F \hookrightarrow M \stackrel{\pi}{\rightarrow} B$ um fibrado com $M, F, B$ e grupo estrutural $G$ compactos. Assuma que: 
1. Uma órbita principal de $G$ em $F$ tem grupo fundamental finito,

2. F possua uma métrica $G$-invariante tal que $\operatorname{Ric}_{F^{r e g} / G} \geq 1$.

\section{Então}

1. Existe $\lambda \in(0,1]$, que depende só da geometria da fibra, tal que qualquer função suave $f: M \rightarrow$ $\mathbb{R}$ satisfazendo $\frac{\min _{p \in B} f}{\max _{p \in B} f} \leq \lambda$ é curvatura escalar de uma métrica de submersão completa em $M$, exceto possitivelmente se $f=$ cte $\geq 0$;

2. Se $F$ possui curvatura escalar constante, então toda função suave $f: M \rightarrow \mathbb{R}$ é curvatura escalar de uma métrica de submersão completa $M$, exceto possivelmente se $f=$ cte $\geq 0$.

O Teorema 7.1.1 é uma generalização natural, em técnica e forma, do seguinte tereoma descrito em $[\mathrm{BG}]$ :

Teorema 7.1.2 (Grou). Seja $F \hookrightarrow M \stackrel{\pi}{\rightarrow} B$ um fibrado com $M, F, B$ e grupo estrutural $G$ compactos. Assuma que:

1. F admite uma métrica $G$-invariante com curvatura seccional positiva;

2. A ação de $G(\operatorname{dim} G \geq 2)$ em $F$ possui apenas um tipo de órbita;

Então existe $\lambda \in(0,1)$ tal que toda função $f: C^{\infty}(M) \rightarrow M$ que satsifaça $\frac{\min f}{\max f} \leq \lambda$ é função de curvatura escalar para alguma métrica de submersão em $M$, exceto possivelmente quando $f=$ cte $\geq$ 0 .

A demonstração do Teorema 7.1.1 faz uso direto (e simples) do seguinte resultado de KazdanWarner:

Teorema 7.1.3 (Kazdan-Warner). Seja $(M, g)$ uma variedade riemanniana compacta. Denote por scal $_{g}$ a curvatura escalar de $g$. Seja $f \in C^{\infty}(M)$ uma função suave em $M$. Se existir uma constante $c>0$ tal que

$$
\min c f<\operatorname{scal}_{g}(p)<\max c f, \forall p \in M,
$$

existe uma métrica riemanniana $\widetilde{g}$ em $M$ tal que

$$
\operatorname{scal}_{\widetilde{g}}=f .
$$

Demonstração. Veja [KW75a, Theorem A].

Prova do Teorema 7.1.1. De acordo com a hipótese dada pelo item 2, $F$ admite uma métrica $G$ invariante com curvatura de Ricci positiva (veja por exemplo o Teorema 5.2.1). Considere em $M$ a única métrica riemanniana $g$ cujas fibras são totalmente gedoésicas e isométricas a $F$ (como na demonstração do Teorema 3.1.5). Reforçamos que no caso de fibrados principais essa pode ser assumida uma métrica de conexão com 1-forma $G$-invariante (veja por exemplo a Proposição 4.2.2).

Fixe $p \in B$ e sejam $\left\{e_{i}\right\}_{i=1}^{k}$ uma base ortonogmal para $\mathcal{H}_{p}$ e $\left\{e_{j}\right\}_{j=k+1}^{n}$ uma base ortonormal para $\mathcal{V}_{p}$. Note que $\left\{e_{i}\right\}_{i=1}^{k} \cup\left\{e^{-t} e_{j}\right\}_{j=k+1}^{n}$ é uma base $g_{t}$-ortonormal para $T_{p} M$. Usando as fórmulas dadas pela Proposição 3.2.4 concluímos que a curvatura escalar de $g_{t}$ é dada por

$$
\operatorname{scal}_{t}(p)=\operatorname{scal}_{\mathrm{t}}^{\mathcal{H}}(p)+2 e^{2 t} \sum_{i, j}\left|A_{e_{i}}^{*} e_{j}\right|_{g}^{2}+e^{-2 t} \operatorname{scal}_{F}(p) .
$$

Mais ainda,

$$
\operatorname{scal}_{\mathrm{t}}^{\mathcal{H}}(p)=\operatorname{scal}_{B}(p)\left(1-e^{2 t}\right)+e^{2 t} \operatorname{scal}_{g}^{\mathcal{H}}(p) .
$$

Denote por $s_{t}:=\min _{p \in B} \operatorname{scal}_{t}(p)$ e por $S_{t}:=\max _{p \in B} \operatorname{scal}_{t}(p)$. Então note que

$$
\lambda:=\lim _{t \rightarrow-\infty} \frac{s_{t}}{S_{t}}=\frac{\min _{p \in B} \mathrm{scal}_{F}}{\max _{p \in B} \operatorname{scal}_{F}} \leq 1 .
$$


Assim, dado $\epsilon>0$ pequeno o suficiente, existe $-t<0$ tal que

$$
\frac{\min _{p \in B} f}{\max _{p \in B} f} \leq \lambda<\epsilon+\frac{s_{-t}}{S_{-t}}
$$

ou seja,

$$
1-\epsilon<\left(\frac{s_{-t}}{\min _{p \in B} f}\right)\left(\frac{\max _{p \in B} f}{S_{-t}}\right),
$$

e o resultado segue como consequência do Teorema 7.1.3.

Note que podemos explorar um pouco mais as hipóteses do Teorema 7.1.1 e o resultado do Teorema 5.2.1, obtendo os corolários (compare com [BG, Corolário 2.2.4, 2.3.13 - 2.3.15]):

Corolário 7.1.4. O toro $T^{n}$ não admite ações por grupos de Lie compactos $G$ tais que

1. Uma órbita principal da ação por $G$ em $T^{n}$ possui grupo fundamental finito;

2. $\operatorname{Ric}_{\left(T^{n}\right)^{r e g} / G} \geq 1$.

Demonstração. Isso segue do fato que nessas condições $T^{n}$ admitira uma métrica de curvatura de Ricci positiva (veja o Teorema 5.2.1). Em particular, essa métrica teria curvatura escalar positiva, o que não é possível uma vez que toda métrica de curvatura escalar não negativa em um toro necessariamente é plana (veja [GL80]).

Eells e Kuiper em [EK62] efetuaram o cálculo do número de 7 (respectivemanete 15)-esferas exóticas que se exprimem como fibrados de esfera sobre esferas padrões. Portanto, no caso em que $G=O(n+1), n=7,15, F=S^{n}$, o Teorema 7.1.1 diz que:

Teorema 7.1.5. Das 28 (respec. 16.256) classes de difeomorfismos de 7 (resp. 15)-esferas exóticas, 16 (resp. 4.096) são tais que toda funçao real suave $f: \Sigma^{7} \rightarrow \mathbb{R}$ (resp. $f: \Sigma^{15} \rightarrow \mathbb{R}$ é uma função curvatura escalar de alguma métrica riemanniana em $\Sigma^{7}$ (resp. $\left.\Sigma^{15}\right)$, exceto possivelmente quando $f=$ cte $\geq 0$.

De maneira geral, pode-se aplicar o Teorema 7.1.1 para os exemplos construídos no Capítulo 6, obtendo como possível aplicação:

Teorema 7.1.6. Seja $S^{7} \hookrightarrow \Sigma^{7} \times{ }_{S^{3}} S^{7} \rightarrow \Sigma^{7}$ o fibrado associado obtido por meio do diagrama (4.6). Então qualquer função suave em $\Sigma^{7} \times_{S^{3}} \Sigma^{7}$ é função de curvatura escalar para alguma métrica nesse, exceto possivelmente se $f=$ cte $\geq 0$. Analogamente, se $\Sigma^{8}$ é a única esfera exótica de dimensão 8 , o resultado vale para o fibrado $S^{8} \hookrightarrow \Sigma^{8} \times{ }_{S^{3}} S^{8} \rightarrow \Sigma^{8}$.

Na seção seguinte estaremos interessados em variedades $(M, g)$ cujas fibras possuem curvatura escalar com sinal misto.

\subsection{Fibrados Topológicos com fibras com curvatura escalar com sinal misto}

Teorema 7.2.1. Seja $\pi:\left(F, g_{F}\right) \hookrightarrow(M, g) \rightarrow(B, h)$ uma submersão riemanniana com $M$ fechada e conexa e $F$ compacta e conexa. Assuma que:

1. $\min _{F} \operatorname{scal}_{F} \leq 0$,

2. $\left(F, g_{F}\right)$ seja subvariedade minima de $(M, g)$, 
3. Se A denota o tensor de Gray-O'Neill da submersão $\pi$, então

$$
\max _{M}\left\{3 \sum_{i, j}\left|A_{e_{i}} e_{j}\right|^{2}-2 \sum_{i, r}\left|A_{e_{i}}^{*} v_{r}\right|^{2}\right\} \leq 0
$$

onde $\left\{e_{i}\right\}$ é uma base g-ortonormal para $\mathcal{H}$ e $\left\{v_{i}\right\}$ é uma base g-ortonormal para $\mathcal{V}$.

Defina as constantes

$$
b_{k}:=\frac{k+1}{8 k}, c_{k}:=\frac{(k+1)^{2}}{8(k-1) k}, \theta:=\frac{2(k-1)}{k+1} .
$$

Então qualquer função $f \in C^{\infty}(M ; \mathbb{R})$ satisfazendo

$$
\frac{\lambda_{1}}{2+\epsilon}-b_{k} \max _{M}\left(f-\mathrm{scal}_{g}+\operatorname{scal}_{F}\right)+c_{k} \min _{F} \operatorname{scal}_{F}(\operatorname{vol}(B))^{2 / \theta-1}+b_{k} \min _{M} \delta A>0,
$$

para algum $\epsilon>0$, onde $\lambda_{1}$ denota o primeiro autovalor positivo de $-\Delta_{B}$, é função de curvatura escalar para alguma métrica de submersão em $M$.

No caso em que $M$ é isométrica a um produto, uma vez que as fibras são totalmente geodésicas e $A \equiv 0$, obtemos:

Teorema 7.2.2. Seja $M=\left(B^{n} \times F^{k}, g_{B} \times g_{F}\right)$ uma variedade riemanniana fechada e conexa. Defina as constantes

$$
b_{k}:=\frac{k+1}{8 k}, c_{k}:=\frac{(k+1)^{2}}{8(k-1) k}, \theta:=\frac{2(k-1)}{k+1} .
$$

Suponha que $\min _{F} \operatorname{scal}_{F} \leq 0$. Então qualquer função suave $f \in C^{\infty}(M ; \mathbb{R})$ satisfazendo

$$
\frac{\lambda_{1}}{2+\epsilon}-b_{k} \max _{B}\left(f-\operatorname{scal}_{B}\right)+c_{k} \min _{F} \operatorname{scal}_{F}(\operatorname{vol}(B))^{2 / \theta-1}>0,
$$

para algum $\epsilon>0$, onde $\lambda_{1}$ denota o primeiro autovalor positivo de $-\Delta_{B}$, é função de curvatura escalar para alguma métrica de submersão em $M$.

Os Teoremas 7.2.1 e 7.2.2 são uma generalização natural do problema de preescrever curvatura escalar constante via produtos verticais, amplamente discutidos em [DD87], [EYTK96]. Sob a hipótese de $M$ isométrica a um produto e $B$ com curvatura de Ricci positiva, conseguimos outra condição.

Corolário 7.2.3. Seja $M^{n+k}=\left(B^{n} \times F^{k}, g_{B} \times g_{F}\right)$. Assuma que $\operatorname{Ric}\left(g_{B}\right) \geq(n-1)$ e que $\min _{F} \operatorname{scal}_{F} \leq 0$. Então qualquer função suave $f: M \rightarrow \mathbb{R}$ tal que

$$
n\left(\frac{8 k}{(2+\epsilon)(k+1)}+(n-1)\right)>\max _{B} f+\frac{c_{k}}{b_{k}} \min _{F} \operatorname{scal}_{F}(\operatorname{vol}(B))^{2 / \theta-1},
$$

para algum $\epsilon>0$, é função de curvatura escalar para alguma métrica de submersão em $M$.

Em particular, se $\min _{F} \operatorname{scal}_{F}=0$ a equação (7.4) se reduz a

$$
\frac{8 k n}{(2+\epsilon)(k+1)}+n(n-1)>\max _{M} f \text {. }
$$

Nossa abordagem para a demonstração dos teoremas supracitados se dá a partir de métodos variacionais, e segue de perto o trabalho [EYTK96]. Nesse, os autores lidam essencialmente com o caso em que a variedade $F$ possui curvatura escalar constante, $\operatorname{scal}_{F}=c$, considerando os casos $c>$ $0, c<0, c=0$. Nas hipóteses dos teoremas aqui apresentamos requeremos que $\min _{F} \operatorname{scal}_{F} \leq 0$. Daí, se supusermos que $F$ tenha curvatura escalar constante, essa deve ser essencialmente não positiva. No entanto, o caso que $\operatorname{scal}_{F}=c>0$ já é englobado pelo Teorema 7.1.1. Ademais, justificamos a 
relevância dos nossos resultados pelo fato de que as hipóteses são facilmente verificáveis e não são muito restritivas. Note por exemplo que no caso em que $M$ é um produto, não há restrições para o comportamento de $f$ ao longo de $F$. Ainda mais, no caso em que a base possui curvatura de Ricci positiva, qualquer função com máximo limitado pela dimensão de $M$ é função de curvatura escalar positiva para alguma métrica de submersão.

A fim de bem motivar a demonstração do Teorema 7.2.1, comecemos por demonstrar o Teorema 7.2.2. Seja $f: B \times F \rightarrow \mathbb{R}$ uma função suave. O Teorema 7.2.2 é provado utilizando técnicas de métodos variacionais para garantir existência de solução para a seguinte equação diferencial parcial:

$$
\Delta_{B} u+\frac{k+1}{4 k}\left(f-\operatorname{scal}_{B}\right) u-\frac{k+1}{4 k} u^{\frac{k-3}{k+1}} \operatorname{scal}_{F}=0,
$$

Para fundamentar a abordagem variacional, assumimos inicialmente hipóteses mínimas de regularidae. A saber, comecemos por assumir que $f, \operatorname{scal}_{B}, \operatorname{scal}_{F}$ sejam funções contínuas. Se $H^{1}(B)$ denota $W^{1,2}(B)$-espaço de Sobolev em $B$ (veja o Apêndice A) definimos o funcional $J: H^{1}(B) \rightarrow \mathbb{R}$ por

$$
\begin{array}{r}
J(u)=\frac{1}{2} \int_{B}|\nabla u|^{2}-\left(\frac{k+1}{8 k}\right) \int_{B}\left(f-\operatorname{scal}_{B}\right) u^{2}+\frac{(k+1)^{2}}{8(k-1) k} \int_{B} \operatorname{scal}_{F} u^{2(k-1) /(k+1),}, \\
\forall u \in H^{1}(B) .
\end{array}
$$

Defina as constantes:

$$
b_{k}:=\frac{k+1}{8 k}, c_{k}:=\frac{(k+1)^{2}}{8(k-1) k}, \theta:=2 \frac{k-1}{k+1} .
$$

O funcional (7.7) se escreve como:

$$
J(u)=\frac{1}{2} \int_{B}|\nabla u|^{2}-b_{k} \int_{B}\left(f-\operatorname{scal}_{B}\right) u^{2}+c_{k} \int_{B} u^{\theta} \operatorname{scal}_{F} .
$$

Observação. Note que $0<\theta \leq 2$.

A solução $u$ procurada será obtida como ponto de mínimo para $J$ quando restrita ao conjunto $\mathbf{M}:=\left\{u \in H^{1}(B): \int_{B} u^{\theta} \geq 1\right\}$. Precisamente, o Teorema 7.2.2 segue como consequência do seguinte lema.

Lema 18. Seja $\epsilon_{0}>0$ arbitrariamente pequeno e defina $\mathbf{M}:=\left\{u \in H^{1}(B): u \geq \epsilon_{0}, \int_{B} u^{\theta} \geq 1\right\}$. Assuma que $f, \mathrm{scal}_{B}, \mathrm{scal}_{F}$ sejam funções contínuas e que $\min _{F} \mathrm{scal}_{F} \leq 0$. Seja $\lambda_{1}$ o primeiro autovalor positivo de $-\Delta_{B}$. Assuma que exista $\epsilon>0$ tal que seguinte relação é satisfeita,

$$
\alpha:=\frac{\lambda_{1}}{2+\epsilon}-b_{k} \max _{B}\left(f-\operatorname{scal}_{B}\right)+c_{k} \min _{F} \operatorname{scal}_{F}(\operatorname{vol}(B))^{2 / \theta-1}>0 .
$$

Então,

1. Existe uma constante $c_{0}>0$ tal que $\left.J\right|_{\mathbf{M}}>c_{0}$,

2. $\left.J\right|_{\mathcal{M}}$ é coercivo,

3. $\left.J\right|_{\mathcal{M}}$ é fracamente semi-contínuo inferiormente,

4. $M$ é fracamente fechado.

Observação. A necessidade de existência de $\epsilon$ na hipótese do Lema 18 se dá com o intuito de garantir que $\left.J\right|_{\mathcal{M}}$ seja coercivo.

A equação (7.6) é obtida como consequência do seguinte lema. 
Lema 19. Sejam $\left(B, g_{B}\right)$ e $\left(F, g_{F}\right)$ variedades riemannianas e $\phi: B \rightarrow \mathbb{R}$ uma função suave. Denote por $\widetilde{g}$ a métrica riemanniana em $B \times_{e^{2 \phi}} F$. Então a curvatura escalar de $g$ tem a seguinte expressão

$$
\widetilde{\mathrm{scal}}=\operatorname{scal}_{B}+e^{-2 \phi} \operatorname{scal}_{F}-k(k-1)|\nabla \phi|^{2}-2 k|\nabla \phi|^{2}-2 k \Delta_{B} \phi .
$$

Demonstração. Considere uma base $g_{B}$-ortonormal $\left\{w_{j}\right\}$ e uma base $g_{F}$-ortonormal $\left\{v_{i}\right\}$. Assim, o conjunto

$$
\left\{w_{j}\right\} \cup\left\{e^{-\phi} v_{i}\right\}
$$

forma uma base $\widetilde{g}$-ortonormal para $T(B \times F)$.

Utilizando as equacões fornecidas pela Proposição 3.2.3,

$$
\begin{aligned}
& \widetilde{K}\left(e^{-\phi} v_{i}, e^{-\phi} v_{j}\right)=e^{-2 \phi} K_{F}\left(v_{i}, v_{j}\right)-|\nabla \phi|^{2}, \\
& \widetilde{K}\left(w_{j}, e^{-\phi} v_{i}\right)=-d \phi\left(w_{j}\right)^{2}-\operatorname{Hess} \phi\left(w_{j}, w_{j}\right) .
\end{aligned}
$$

Assuma que $\left\{w_{j}\right\}$ seja tal que $d \phi\left(w_{1}\right)=|\nabla \phi|, d \phi\left(w_{j}\right)=0, \forall j \geq 2$. Então a curvatura escalar de $\widetilde{g}$ é dada por

$$
\widetilde{\mathrm{scal}}=\operatorname{scal}_{B}+e^{-2 \phi} \mathrm{scal}_{F}-k(k-1)|\nabla \phi|^{2}-2 k\left\{|\nabla \phi|^{2}+\Delta_{B} \phi\right\} \text {. }
$$

Seja $f \in C^{\infty}(B \times F ; \mathbb{R})$. Consideramos inicialmente a seguinte EDP:

$$
\operatorname{scal}_{B}+e^{-2 \phi} \operatorname{scal}_{F}-f=k(k-1)|\nabla \phi|^{2}+2 k\left\{|\nabla \phi|^{2}+\Delta_{B} \phi\right\} .
$$

Note que se $\phi=\log \varphi$ então $\nabla \phi=\frac{1}{\varphi} \nabla \varphi$. Consequentemente

$$
\Delta_{B} \phi=-\frac{1}{\varphi^{2}}|\nabla \varphi|^{2}+\frac{1}{\varphi} \Delta_{B} \varphi
$$

Assim, como $e^{-2 \phi}=\varphi^{-2}$ então

$$
k(k-1)|\nabla \phi|^{2}+2 k e^{-2 \phi}\left\{|\nabla \phi|^{2}+\Delta_{B} \phi\right\}=\frac{k(k-1)}{\varphi^{2}}|\nabla \varphi|^{2}+2 k\left\{\frac{1}{\varphi^{2}}|\nabla \varphi|^{2}-\frac{1}{\varphi^{2}}|\nabla \varphi|^{2}+\frac{1}{\varphi} \Delta_{B} \varphi\right\} .
$$

Logo, concluímos que

$$
\operatorname{scal}_{B}+\varphi^{-2} \operatorname{scal}_{F}-f=\frac{k(k-1)}{\varphi^{2}}|\nabla \varphi|^{2}+\frac{2 k}{\varphi} \Delta_{B} \varphi
$$

Introduzindo a mudança de variáveis $\varphi=u^{\frac{2}{k+1}}$ obtemos

$$
\nabla \varphi=\frac{2}{k+1} u^{\frac{1-k}{k+1}} \nabla u, \Delta_{B} \varphi=\left(2 \frac{(1-k)}{(k+1)^{2}} u^{-\frac{2 k}{k+1}}|\nabla u|^{2}+\frac{2}{k+1} u^{\frac{1-k}{k+1}} \Delta_{B} u\right) .
$$


Substituindo esses termos na equação (7.12) temos

$$
\begin{aligned}
\operatorname{scal}_{B}+u^{-\frac{4}{k+1}} \operatorname{scal}_{F}-f=4 \frac{k(k-1)}{(k+1)^{2}} u^{-\frac{4}{k+1}} u^{\frac{2(1-k)}{k+1}}|\nabla u|^{2}+ \\
2 k u^{\frac{-2}{k+1}}\left(2 \frac{(1-k)}{(k+1)^{2}} u^{-\frac{2 k}{k+1}}|\nabla u|^{2}+\frac{2}{k+1} u^{\frac{1-k}{k+1}} \Delta_{B} u\right) \\
\operatorname{scal}_{B}+u^{\frac{-4}{k+1}} \mathrm{scal}_{F}-f=4 \frac{k(k-1)}{(k+1)^{2}} u^{-2}|\nabla u|^{2} \\
+4 \frac{k(1-k)}{(k+1)^{2}} u^{-2}|\nabla u|^{2}+\frac{4 k}{k+1} u^{-1} \Delta_{B} u \\
=\frac{4 k}{k+1} u^{-1} \Delta_{B} u .
\end{aligned}
$$

Passamos agora à demonstração do Lema 18.

Demonstração do Lema 18. 1. Seja $u \in$ M. Recorde a desigualdade de Poincaré,

$$
\int_{B}|\nabla u|^{2} \geq \lambda_{1} \int_{B} u^{2}
$$

Pelas hipóteses sobre $f, \operatorname{scal}_{B}, \operatorname{scal}_{F}$ temos

$$
J(u) \geq \frac{1}{2} \int_{B}|\nabla u|^{2}-b_{k} \max _{B}\left(f-\operatorname{scal}_{B}\right) \int_{B} u^{2}+c_{k} \min _{F} \operatorname{scal}_{F} \int_{B} u^{\theta} .
$$

Como $B$ é compacta e $\theta \leq 2$ a Desigualdade de Höder (veja a equação (A.2)) diz que existe uma imersão contínua $L^{2}(B) \hookrightarrow L^{\theta}(B)$. Em particular

$$
\left(\int_{B} u^{\theta}\right)^{2 / \theta} \leq(\operatorname{vol}(B))^{2 / \theta-1} \int_{B} u^{2} .
$$

Como $\int u^{\theta} \geq 1$ e $\theta \leq 2$ então

$$
\int_{B} u^{\theta} \leq(\operatorname{vol}(B))^{2 / \theta-1} \int_{B} u^{2}
$$

Da hipótese $\min _{F} \operatorname{scal}_{F} \leq 0$ segue que

$$
c_{k} \min _{F} \operatorname{scal}_{F} \int_{B} u^{\theta} \geq c_{k} \min _{F} \operatorname{scal}_{F}(\operatorname{vol}(B))^{2 / \theta-1} \int_{B} u^{2} .
$$

Portanto

$$
J(u) \geq \frac{1}{2} \int_{B}|\nabla u|^{2}-b_{k} \max _{B}\left(f-\operatorname{scal}_{B}\right) \int_{B} u^{2}+c_{k} \min _{F} \operatorname{scal}_{F}(\operatorname{vol}(B))^{2 / \theta-1} \int_{B} u^{2} .
$$

Pela equação (7.13) temos

$$
J(u) \geq\left(\frac{\lambda_{1}}{2}-b_{k} \max _{B}\left(f-\operatorname{scal}_{B}\right)+c_{k} \min _{F} \operatorname{scal}_{F}(\operatorname{vol}(B))^{2 / \theta-1}\right) \int_{B} u^{2} .
$$

Por hipótese

$$
\frac{\lambda_{1}}{2}-b_{k} \max _{B}\left(f-\operatorname{scal}_{B}\right)+c_{k} \min _{F} \operatorname{scal}_{F}(\operatorname{vol}(B))^{2 / \theta-1}>0 .
$$

De acoro com a equação (7.14) e com a definição de $\mathbf{M}$, existe $c_{0}>0$ tal que $J(u) \geq c_{0}, \forall u \in$ M. 
2. Para provar a coercividade note que como

$$
J(u) \geq \frac{1}{2} \int_{B}|\nabla u|^{2}-\left(b_{k} \max _{B}\left(f-\operatorname{scal}_{B}\right)-c_{k} \min _{F} \operatorname{scal}_{F}(\operatorname{vol}(B))^{2 / \theta-1}\right) \int_{B} u^{2}
$$

pela equação (7.9)

$$
J(u)>\frac{1}{2} \int_{B}|\nabla u|^{2}-\frac{\lambda_{1}}{2+\epsilon} \int_{B} u^{2} .
$$

Daí, pela desigualdade de Poincaré (equação (7.13))

$$
\begin{gathered}
J(u)>\lambda_{1}\left(\frac{1}{2}-\frac{1}{2+\epsilon}\right) \int_{B}|\nabla u|^{2} \\
J(u)>\frac{\lambda_{1} \epsilon}{2(2+\epsilon)} \int_{B}|\nabla u|^{2},
\end{gathered}
$$

de onde segue que $J$ é coercivo.

3. Para ver que $J$ é fracamente semi-contínuo inferiormente, note que pelo Teorema de Kondrachov (Teorema A.2.1) $H^{1}(B)$ mergulha compactamente em $L^{2}(B)$. Daí, seja $\left\{u_{n}\right\} \subset \mathbf{M}$ convergindo fracamente a $u \in H^{1}(B)$. Então $u_{n} \rightarrow u \in L^{2}(B)$. Por compacidade de $B$ e pelo fato que $\operatorname{scal}_{B}, \operatorname{scal}_{F}$ e $f$ são contínuas, lembrando a identidade clássica para convergência fraca

$$
\int_{B}|\nabla u|^{2} \leq \lim \inf _{n \rightarrow \infty} \int_{B}\left|\nabla u_{n}\right|^{2}
$$

por fim, de acordo com o Teorema da Convergência Dominada,

$$
\begin{aligned}
\lim \inf _{n \rightarrow \infty} J\left(u_{n}\right) & =\lim \inf _{n \rightarrow \infty}\left(\int_{M}\left|\nabla u_{n}\right|^{2}-b_{k} \int_{B}\left(f-\operatorname{scal}_{B}\right) u_{n}^{2}+c_{k} \int_{B} u_{n}^{\theta} \operatorname{scal}_{F}\right) \\
& \geq \int_{M}|\nabla u|^{2}-b_{k} \int_{B}\left(f-\operatorname{scal}_{B}\right) u^{2}+c_{k} \int_{B} u^{\theta} \operatorname{scal}_{F}=J(u) .
\end{aligned}
$$

4. Novamente isso segue do fato que $H^{1}(B)$ mergulha compactamente em $L^{2}(B)$ e esse admite imersão contínua em $L^{\theta}(B)$. Assim, se $\left\{u_{n}\right\} \subset \mathbf{M}$ converge fracamente para $u \in H^{1}(B)$ então $\left\{u_{n}\right\}$ converge forte a $u$ em $L^{\theta}(M)$, de onde

$$
1 \leq \lim _{n \rightarrow \infty} \int_{B} u_{n}^{\theta}=\int_{B} u^{\theta} .
$$

Por outro lado, existe uma subsequência $\left\{u_{n_{j}}\right\}$ convergindo pontualmente quase sempre para $u$. Daí, $u=\lim _{j \rightarrow \infty} u_{n_{j}} \geq \epsilon_{0}$, o que conclui a demonstração.

Demonstração do Teorema 7.2.2. Pelo Lema 18 e pelo Teorema de Weierstrass (Teorema A.3.1) segue que $\left.J\right|_{\mathbf{M}}$ possui um ponto crítico. Assim, para qualquer qualquer $v \in H^{1}(B)$ temos

$$
\int_{B}\left(\Delta_{B} u+\frac{k+1}{4 k}\left(f-\operatorname{scal}_{B}\right) u-\frac{k+1}{4 k} \operatorname{scal}_{F} u^{k-3 / k+1}\right) v=0,
$$

onde onde $u$ é uma solução fraca (veja a discussão no Apêndice A). Se assumirmos que $f, \operatorname{scal}_{B}, \operatorname{scal}_{F}$ sejam suaves, pela teoria clássica de regularidade para equações elípticas (veja [Aub98, Theorem 3.58 , p. 87]) segue que $u$ é uma função suave.

Passemos agora ao Teorema 7.2.1.

Lema 20. Seja $(M, g)$ uma variedade riemanniana munida de uma folheação regular riemanniana $\mathcal{F}$. Seja $\widetilde{g}$ uma métrica de produto vertical geral em $M$ via função $u^{\frac{4}{k+1}}$ onde $u: M \rightarrow \mathbb{R}$ é uma 
função suave com gradiente básico. Então a curvatura escalar de $\widetilde{g}$ é dada por

$$
\begin{aligned}
\widetilde{\text { scal }}=\operatorname{scal}_{g}-\frac{4 k}{k+1} & u^{-1} \Delta_{B} u+(4+2(k-1)) \frac{2}{k+1} u^{-1} d u(H) \\
& +\left(u^{-\frac{4}{k+1}}-1\right) \operatorname{scal}_{F}+\left(1-u^{\frac{4}{k+1}}\right)\left(3 \sum_{i, j}\left|A_{e_{i}} e_{j}\right|^{2}-2 \sum_{i, r}\left|A_{e_{i}}^{*} v_{r}\right|^{2}\right) .
\end{aligned}
$$

Demonstração. Tendo em mente as fórmulas da Proposição 3.2.1, sejam $T_{i}=e^{-\phi} v_{i}, v_{i} \in \mathcal{V} \mathrm{e}$ $X \in \mathcal{H}$. Então

$$
\begin{aligned}
& \widetilde{K}\left(e^{-\phi} v_{1}, e^{-\phi} v_{2}\right)=\left(e^{-2 \phi}-1\right) K_{F}\left(v_{1}, v_{2}\right)+K_{g}\left(v_{1}, v_{2}\right)-|\nabla \phi|^{2}+d \phi\left(\sigma\left(v_{1}, v_{1}\right)+\sigma\left(v_{2}, v_{2}\right)\right), \\
& \widetilde{K}\left(X, e^{-\phi} v\right)=K_{g}(X, v)-\left(1-e^{2 \phi}\right)\left|A_{X}^{*} v\right|^{2}-\operatorname{Hess} \phi(X, X)-d \phi(X)^{2}+2 d \phi(X) g\left(S_{X} v, v\right) .
\end{aligned}
$$

Tome uma base $g$-ortonormal $\left\{e_{i}\right\}$ para $\mathcal{H}$. Assim

$$
\begin{gathered}
\sum_{i, j} \widetilde{K}\left(e_{i}, e_{j}\right)=\left(1-e^{2 \phi}\right) \mathrm{scal}_{B}+e^{2 \phi} \mathrm{scal}^{\mathcal{H}}, \\
\sum_{r, s} \widetilde{K}\left(e^{-\phi} v_{r}, e^{-\phi} v_{s}\right)=\left(e^{-2 \phi}-1\right) \operatorname{scal}_{F}+\mathrm{scal}^{\mathcal{V}}-k(k-1)|\nabla \phi|^{2}+2(k-1) d \phi(H) \\
2 \sum_{i, r} \widetilde{K}\left(e^{-\phi} v_{r}, e_{i}\right)=2 \sum_{i, r} K\left(e_{i}, v_{r}\right)-2\left(1-e^{2 \phi}\right) \sum_{i, r}\left|A_{e_{i}}^{*} v_{r}\right|^{2}-2 k\left(\Delta_{B} \phi+|\nabla \phi|^{2}\right)+4 \sum_{r, i} d \phi\left(e_{i}\right) g\left(S_{e_{i}} v_{r}, v_{r}\right) .
\end{gathered}
$$

Assuma que $d \phi\left(e_{1}\right)=|\nabla \phi|$, i.e, $e_{1}=\frac{\nabla \phi}{|\nabla \phi|}, d \varphi\left(e_{i}\right)=0, i \geq 2$. Assim,

$$
4 \sum_{r, i} d \phi\left(e_{i}\right) g\left(\nabla_{e_{i}} v_{r}, v_{r}\right)=4 \operatorname{tr} S_{\nabla \varphi}=4 d \varphi(H)
$$

De onde concluímos que

$$
\begin{aligned}
\widetilde{\operatorname{scal}}=\operatorname{scal}_{g}-2\left(1-e^{2 \phi}\right) \sum_{i, r} & \left|A_{e_{i}}^{*} v_{r}\right|^{2}+\left(1-e^{2 \phi}\right)\left(\operatorname{scal}_{B}-\operatorname{scal}^{\mathcal{H}}\right)+\left(e^{-2 \phi}-1\right) \operatorname{scal}_{F} \\
& -k(k-1)|\nabla \phi|^{2}-2 k\left(\Delta_{B} \phi+|\nabla \phi|^{2}\right)+(4+2(k-1)) d \phi(H) .
\end{aligned}
$$

Logo, como a equação (7.24) difere da equação (7.11) somente pelos termos

$$
2\left(1-e^{2 \phi}\right) \sum_{i, r}\left|A_{e_{i}}^{*} v_{r}\right|^{2}+(4+2(k-1)) d \phi(H)
$$

fazendo as mudanças $\phi=\log \varphi$ e $\varphi=u^{\frac{2}{k+1}}$ concluímos que

$$
\begin{aligned}
\widetilde{\text { scal }}=\operatorname{scal}_{g}-\frac{4 k}{k+1} & u^{-1} \Delta_{B} u+(4+2(k-1)) \frac{2}{k+1} u^{-1} d u(H) \\
& +\left(u^{-\frac{4}{k+1}}-1\right) \operatorname{scal}_{F}+\left(1-u^{\frac{4}{k+1}}\right)\left(\operatorname{scal}_{B}-\operatorname{scal}^{\mathcal{H}}-2 \sum_{i, r}\left|A_{e_{i}}^{*} v_{r}\right|^{2}\right) .
\end{aligned}
$$

Lema 21. Seja $\left(F, g_{F}\right) \hookrightarrow(M, g) \rightarrow(B, h)$ uma submersão riemanniana com $M$ fechada e conexa $e\left(F, g_{F}\right)$ compacta, conexa e minima. Seja $\Delta_{M}$ o operador de Laplace em $M$. Então a restrição de $\Delta_{M}$ às funções básicas define um operador uniformemente elíptico. 
Demonstração. Se $u: M \rightarrow \mathbb{R}$ é uma função básica então $\nabla u$ é um campo básico. Assim, $u$ é constante ao longo de cada fibra $F$. Escolha uma coleção de abertos $\left\{U_{n}\right\} \subset M$ que trivializam a folheação a submersão, isto é,

$$
U_{n}=B_{n} \times F, B_{n} \subset B
$$

Então, como $u$ é função básica,

$$
\left.u\right|_{U_{n}}(p)=u(b, f)=u\left(b, f^{\prime}\right), \forall f, f^{\prime} \in F, \forall b \in B_{n} .
$$

Se $\left\{\psi_{n}\right\}$ denote uma partição da unidade subordinada à cobertura $\left\{B_{n}\right\}$, então $u=\sum_{n} \psi_{n} u$ e temos bem definida uma injeção

$$
\begin{gathered}
\zeta: H^{1}(M) \rightarrow H^{1}(B) \\
u \mapsto v,
\end{gathered}
$$

onde $v=\sum_{n} v_{n}, v_{n}(b)=\psi_{n} u(b, f), \forall b \in B_{n}$.

Por outro lado, como $\left(F, g_{F}\right)$ é mínima, identificando $\zeta u=u$ temos que $\Delta_{B} u=\Delta_{M} u$. Portanto, como $\Delta_{B}$ é fortemente elíptico, o resultado segue.

Demonstração do Teorema 7.2.1. Defina

$$
\delta A:=3 \sum_{i, j}\left|A_{e_{i}} e_{j}\right|^{2}-2 \sum_{i, j}\left|A_{e_{i}}^{*} v_{r}\right|^{2},
$$

onde $\left\{e_{i}\right\}$ é uma base $g$-ortonormal para $\mathcal{H}$ e $\left\{v_{r}\right\}$ é uma base $g$-ortonormal para $\mathcal{V}$. Tendo em mente o Lema 21 , dada $f: M \rightarrow \mathbb{R}$, estudaremos o problema elíptico

$$
\left(\frac{k+1}{4 k}\right) u\left(f-\operatorname{scal}_{g}\right)=-\Delta_{B} u+\left(\frac{k+1}{4 k}\right)\left\{\left(u^{\frac{k-3}{k+1}}-u\right) \operatorname{scal}_{F}+\left(u-u^{\frac{k+5}{k+1}}\right) \delta A\right\}
$$

Lembrando que $\theta=\frac{2(k-1)}{k+1}$ e que $\frac{k+1}{4 k}=2 b_{k}$, defina $\gamma:=\frac{2 k+6}{k+1}$. Seja $\epsilon_{0}>0$ arbitrariamente pequeno e defina o conjunto

$$
\mathbf{M}^{b}:=\left\{u \in H^{1}(M): u \geq \epsilon_{0}, \nabla u \text { é básico e } \int_{B} u^{\theta} \geq 1\right\}
$$

onde $\theta=\frac{2(k-1)}{k+1}$. Considere o seguinte funcional $J$ definido em $\mathbf{M}^{b}$ :

$$
\begin{aligned}
& J(u):=\frac{1}{2} \int_{B}|\nabla u|^{2} \\
& +\left(\frac{k+1}{4 k}\right) \int_{B}\left\{-\frac{u^{2}}{2}\left(f-\operatorname{scal}_{g}\right)+\left(\frac{k+1}{2(k-1)} u^{\frac{2(k-1)}{k+1}}-\frac{1}{2} u^{2}\right) \operatorname{scal}_{F}+\left(\frac{u^{2}}{2}-\frac{k+1}{2 k+6} u^{\frac{2 k+6}{k+1}}\right) \delta A\right\} .
\end{aligned}
$$

Então convenientemente reescrevemos $J$ como

$$
J(u)=\frac{1}{2} \int_{B}|\nabla u|^{2}+2 b_{k} \int_{B}\left\{\left(\operatorname{scal}_{g}+\delta A-\operatorname{scal}_{F}-f\right) \frac{u^{2}}{2}+\operatorname{scal}_{F} \theta^{-1} u^{\theta}-\delta A \gamma^{-1} u^{\gamma}\right\}
$$

Vale notar que $\frac{2}{3} \leq \theta \leq 2,2 \leq \gamma \leq \frac{10}{3}, k \geq 2$. Assuma que

1. $\max _{B} \delta A \leq 0$,

2. $\min _{F} \operatorname{scal}_{F} \leq 0$.

Então, 


$$
\begin{aligned}
J(u) \geq \frac{1}{2} \int_{B}|\nabla u|^{2}+b_{k} & \left(\min _{M} \operatorname{scal}_{g}-\max _{M} f-\max _{M} \operatorname{scal}_{F}+\min _{M} \delta A\right) \int_{B} u^{2} \\
& +2 b_{k} \theta^{-1} \min _{F} \operatorname{scal}_{F}(\operatorname{vol}(B))^{2 / \theta-1} \int_{B} u^{2}-2 b_{k} \max _{M} \delta A \gamma^{-1} \int_{B} u^{\gamma}
\end{aligned}
$$

onde o penúltimo termo segue da equação (7.14).

Pela desigualdade de Hölder aplicada à imersão contínua $L^{\gamma}(B) \hookrightarrow L^{2}(B)$ temos

$$
\left(\int_{B} u^{2}\right)^{\frac{\gamma}{2}} \leq \operatorname{vol}(B)^{\frac{\gamma}{2}-1} \int_{B} u^{\gamma} .
$$

Explorando a equação (7.31), a desigualdade de Poincaré e a equação (7.32) nos dizem que

$$
\begin{array}{r}
J(u) \geq\left\{\frac{\lambda_{1}}{2}-b_{k} \max _{M}\left(f-\operatorname{scal}_{g}+\operatorname{scal}_{F}\right)+c_{k} \min _{F} \operatorname{scal}_{F}(\operatorname{vol}(B))^{2 / \theta-1}+b_{k} \min _{M} \delta A\right\} \int_{B} u^{2} \\
-2 b_{k}\left\{\gamma^{-1} \operatorname{vol}(B)^{1-\frac{\gamma}{2}} \max _{M} \delta A\right\}\left(\int_{B} u^{2}\right)^{\frac{\gamma}{2}} .
\end{array}
$$

Logo, a demonstração do Teorema 7.2.1 segue usando a equação (7.33) e uma adaptação simples do Lema 18 ao notar que

$$
-2 b_{k} \gamma^{-1} \operatorname{vol}(B)^{1-\frac{\gamma}{2}} \max _{M} \delta A \geq 0
$$

Demonstração do Corolário 7.2.3. Para demonstração do Corolário 7.2.3 notamos que se $\operatorname{Ric}(B) \geq$ $(n-1)$, então o autovalor $\lambda_{1}$ de $-\Delta_{B}$ satisfaz

$$
\lambda_{1} \geq n
$$

com igualdade se, e somente se, $B$ é isométrica à esfera unitária.

Como $\operatorname{Ric}(B) \geq(n-1)$ implica que $\operatorname{scal}_{g_{B}} \geq n(n-1)$, segue a partir da hipótese dada pela equação 7.4 no Teorema 7.2.2 que uma condição suficiente para existência de solução consiste em:

$$
\frac{n}{2+\epsilon}>b_{k} \max _{B} f-b_{k} n(n-1)-\max _{B} \operatorname{scal}_{F}+c_{k} \min _{F} \operatorname{scal}_{F}(\operatorname{vol}(B))^{2 / \theta-1}, \epsilon>0 .
$$

Em particular, se $\min _{F} \operatorname{scal}_{F}=0$ então essa se traduz como

$$
\frac{8 k n}{(2+\epsilon)(k+1)}+n(n-1)>\max _{B} f .
$$

o que conclui a demonstração. 

ESTRUTURA COMPACTO 


\section{Apêndice A}

\section{Rudimentos de análise}

Neste apêndice destacaremos resultados sobre análise e equações diferenciais parciais que serão úteis ao longo do texto. Não é nossa intenção fazer uma exposição em todos os detalhes, gostaríamos apenas de fornecer o mínimo para contextualizar melhor o leitor sobre alguns pontos do texto. Seguimos como referências [Joy00], [AH10], [Aub98].

\section{A.1 Análise básica em variedades}

\section{A.1.1 Espaços de Lebesgue e Sobolev}

Seja $(M, g)$ uma variedade riemanniana com forma de volume $d \mu(g)$. Para $q \geq 1$ definimos o espaço de Lebesgue $L^{q}(M)$ como sendo o conjunto das funções $f$ localmente integráveis, i.e, integráveis em cada compacto em $M$, e cuja seguinte norma é finita

$$
|f|_{L^{q}}:=\left(\int_{M}|f|^{q} d \mu(g)\right)^{1 / q}<\infty .
$$

Suponha que $r, s, t \geq 1$ e que $\frac{1}{r}=\frac{1}{s}+\frac{1}{t}$. Se $\varphi \in L^{s}(M), \psi \in L^{t}(M)$, então $\varphi \psi \in L^{r}(M)$, e ainda, a seguinte Desigualdade de Hölder é satisfeita:

$$
|\varphi \psi|_{L^{r}} \leq|\varphi|_{L^{s}}|\psi|_{L^{t}}
$$

Note que disso segue que se $M$ é compacta e $s \geq r$, então a inclusão $L^{r}(M) \hookrightarrow L^{q}(M)$ é contínua. Precisamente, note que a função constante igual a 1 é integrável. Em particular, pertence a $L^{t}(M)$. Logo,

$$
|\varphi|_{L^{r}} \leq \operatorname{vol}_{g}(M)^{1 / t}|\varphi|_{L^{s}}
$$

É conveniente estender o conceito de derivada fraca usualmente visto em $\mathbb{R}^{n}$ para variedades. Lembrando que

$$
\nabla^{k}: C^{\infty}(M) \otimes \Omega^{k}(M) \rightarrow C^{\infty}(M) \otimes \Omega^{k+1}(M),
$$

então a derivada fraca de $f \in C^{\infty}(M)$ é a única 1-forma $\nabla f$ que satisfaz

$$
g(\nabla f, \varphi)=\int_{M} f \nabla^{*} \varphi d \mu(g),
$$

para toda 1 -forma $\varphi$ com suporte compacto em $M$; onde $\nabla^{*}$ é o adjunto formal de $\nabla$ segundo a métrica $g$.

Indutivamente,

$$
g\left(\nabla^{k} f, \varphi\right)=g\left(\nabla^{k-1} f, \nabla^{*} \varphi\right) .
$$

Seja $q \geq 1$ e $k$ um inteiro não negativo. Definimos o espaço de Sobolev $W^{k, q}(M)$ como sendo o 
conjunto das funções $f \in L^{q}(M)$ tais que $f$ é $k$-vezes fracamente diferenciável e $\left|\nabla^{j} f\right| \in L^{q}$ para $j \geq k$. Munimos este espaço com a norma

$$
|f|_{L_{k}^{q}}:=\left(\sum_{j=0}^{k} \int_{M}\left|\nabla^{j} f\right|^{q} d \mu(g)\right)^{1 / q},
$$

onde $\nabla$ é a conexão de Levi-Civita de $g$. Mostra-se que $W^{k, q}(M)$ é um espaço de Banach, e $W^{1, q}(M):=H^{q}(M)$ é um espaço de Hilbert. Mais ainda, $W^{k, q}(M)$ é um espaço reflexivo para $q \in(1, \infty)$.

\section{A.2 Kondrakov e Poincaré: inclusões e desigualdades}

Para os resultados do Capítulo 7 precisamos entender como convergências fracas (em espaços de Sobolev) são transportadas para convergências fortes em espaços de Lebesgue. Esses é o conteúdo dos próximo teorema.

Teorema A.2.1 (Teorema de Kondrakov). Sejam $M$ uma variedade riemanniana compacta; $k, l$ inteiros tais que $k \geq l \geq 0$; e $q, r$ números reais com $q, r \geq 1$. Se

$$
\frac{1}{q}<\frac{1}{r}+\frac{k-l}{n}
$$

então o mergulho $W^{k, q}(M) \hookrightarrow W^{l, r}(M)$ é compacto.

Corolário A.2.2. No caso em que $k=1, q=2, l=0$ e $r=2$, então $W^{1,2}(M)$ mergulha compactamente em $L^{2}(M)$.

Teorema A.2.3 (Desigualdade de Poincaré). Sejam $(M, g)$ uma variedade riemanniana fechada e $1 \leq q<\infty$. Então existe $C>0$ tal que

$$
|u|_{L^{q}} \leq C|\nabla u|_{L^{q}}
$$

onde $\nabla$ é a conexão de Levi-Civita de $g$.

Ainda mais, se $q=2$ e $\lambda_{1}$ é o primeiro autovalor (positivo) de $-\Delta$, onde $\Delta$ é o operador de Laplace associado a g, então $C=\sqrt{\lambda_{1}^{-1}}$, e essa é a constante ótima.

\section{A.3 Operadores Diferenciais, equações elípticas e problemas vari- acionais}

\section{A.3.1 Definições gerais}

Sejam $(M, g)$ uma variedade riemanniana e $\nabla$ a conexão de Levi-Civita associada à métrica $g$.

Definição 39. Um operador diferencial parcial, ou simplesmente, operador diferencial, $P$, de ordem $k$, é uma aplicação que leva funções reais u definidas em $M$ em funções reais Pu, também definidas em $M$; que depende de $u$ e de suas $k$-primeiras derivadas. Explicitamente, se $\nabla^{j} u, j \in\{0, \ldots, k\}$ denotam as $k$-primeiras derivadas de $u$ (possivelmente no sentido fraco), então existe uma função real $Q$, continua em cada um de seus argumentos, tal que

$$
(P u)(p)=Q\left(p, u(p), \nabla u(p), \ldots, \nabla^{k} u(p)\right) .
$$

Se $Q$ é suave em cada um de seus argumentos, dizemos que o operador $P$ é operador diferencial suave. Se $P(\alpha u+\beta v)=\alpha P(u)+\beta P(v)$, onde $\alpha, \beta \in \mathbb{R}$, então dizemos que $P$ é linear. Do contrário dizemos que $P$ é não-linear. 
Exemplo. Seja $U \subset \mathbb{R}^{n}$ um conjunto aberto. Um operador diferencial linear de ordem 2 é da forma

$$
(P u)(p)=\sum_{i, j=1}^{n} a_{i j}(p) \frac{\partial^{2} u(p)}{\partial x^{i} \partial x^{j}}+\sum_{k=1}^{n} b^{k}(p) \frac{\partial u(p)}{\partial x^{k}}+c(p) u(p), p=\left(x^{1}, \ldots, x^{n}\right) ;
$$

onde os coeficientes $a_{i j}, b, c$ são funções em $M$ e cuja regularidade define o operador.

Exemplo. Se $P$ é um operador diferencial suave em uma variedade riemanniana $(M, g)$, então claramente $P$ mapeia funções suaves em $M$ em funções suaves em $M$.

Por outro lado, em geral, $P$ decresce regularidade. A saber, se os coeficientes de $P$ são limitados, então

$$
P: W^{k+l, q}(M) \rightarrow W^{l, q}(M) .
$$

Na demonstração do Teorema 7.2.2 precisamos entender operadores elípticos. Esse é o objetivo das próximas linhas.

Definição 40. Sejam $P$ um operador linear de ordem $k$ e $Q$ como em (A.8). Se $Q$ é de classe $C^{1}$ em seus argumentos, então a linearização de $P$ em u é definida via o limite

$$
L_{u} P(v):=\lim _{t \rightarrow 0} \frac{1}{t}(P(u+t v)-P(v)) .
$$

Observamos que em geral, mesmo se o operador $P$ for suave, não há garantias de que sua linearização seja suave. Em verdade, se $u \in C^{k+l}(M)$ e $P$ é de ordem $k$, então $L_{u} P$ tem ordem $l$.

Em coordenadas locais, um operador diferencial linear de ordem $k$ tem a seguinte expressão geral

$$
P u=A^{i_{1} \ldots i_{k}} \nabla_{i_{1} \ldots i_{k}} u+B^{i_{1} \ldots i_{k-1}} \nabla_{i_{1} \ldots i_{k-1}} u+\ldots+K^{i_{1}} \nabla_{i_{1}} u+L u
$$

onde $A, B, K$ são tensores simétricos e $L$ é uma função real. Para cada ponto $p \in M$ e cada $\xi \in T_{x}^{*} M$. Considere a expressão

$$
\sigma_{\xi}(P ; p):=A^{i_{1} \ldots i_{k}} \xi^{i_{1}} \ldots \xi^{i_{k}} .
$$

O símbolo de $P$ é a aplicação

$$
\begin{gathered}
\sigma: T^{*} M \rightarrow \mathbb{R} \\
\sigma:(p, \xi) \mapsto \sigma_{\xi}(P ; p) .
\end{gathered}
$$

\section{A.3.2 Operadores Elípticos e equações diferenciais parciais elípticas}

Definição 41. Um operador diferencial $P$ de ordem $k$ linear é dito elíptico se para todo $(p, \xi) \in$ $T^{*} M-\{0\}$, onde 0 é a seção nula, $\sigma_{\xi}(P ; p) \neq 0$.

Um operador não linear é dito elíptico se sua linearização na direção de toda função for um operador elíptico.

O operador $-\Delta$ é um operador elíptico. Discutiremos a seguir a formulação variacional de alguns problemas elípticos, a fim de ilustrar o procedimento detalhado no Capítulo 7. A referência principal é o livro de Colóquio [Sic15].

Considere o seguinte problema elíptico definido em uma variedade fechada:

$$
-\operatorname{div}(A(p) \nabla u)+g(b, \nabla u)+c(p) u=f(p), \forall p \in M,
$$

onde $A=\left(a_{i j}\right), b=\left(b_{j}\right), c$ são dados e possuem alguma hipótese de regularidade.

Assuma que $u_{0}$ seja uma solução clássica do problema (A.12). Seja $\varphi \in C^{\infty}(M)$. Multiplicando a equação (A.12) por $\varphi$ e integrando, temos

$$
-\int_{M} \varphi \operatorname{div}\left(A \nabla u_{0}\right)+\int_{M} \varphi g\left(b, \nabla u_{0}\right)+\int_{M} c u_{0}=\int_{M} \varphi
$$


Integrando por partes

$$
\begin{aligned}
\int_{M} \varphi f & =\int_{M} g\left(A \nabla u_{0}, \nabla \varphi\right)-\int_{M} \operatorname{div}\left(\varphi A \nabla u_{0}\right)+\int_{M} \varphi g\left(b, \nabla u_{0}\right)+\int_{M} \varphi c u_{0} \\
& =\int_{M} g\left(A \nabla u_{0}, \nabla \varphi\right)+\int_{M} \varphi g\left(b, \nabla u_{0}\right)+\int_{M} \varphi c u_{0} .
\end{aligned}
$$

A ideia agora consiste em tomar a identidade (A.14) como definição de uma solução fraca. Para isso observe que nesta identidade não há nenhuma derivada de ordem maior que um. Ademais, essa equação está definida para funções satisfazem hipóteses bastante gerais, por exemplo: $f \in$ $L^{2}(M), a_{i j}, b_{j}, c \in L^{\infty}(M)$. Essas são precisamente as hipóteses dos resultados no Capítulo 7 .

Uma solução fraca pode ser obtida considerando um funcional associado a equação (A.14) e provando existência de um ponto crítico para esse.

Teorema A.3.1 (Teorema de Weierstrass). Sejam E um espaço de Banach reflexivo e $\emptyset \neq \mathbf{M} \subset E$ um conjunto fracamente fechado. Seja $J: \mathbf{M} \rightarrow \mathbb{R}$ um funcional em $\mathbf{M}$. Assuma que

1. J é fracamente contínuo inferiormente, a saber, se $\left\{u_{n}\right\} \subset \mathbf{M}$ é uma sequência de funções convergindo fracamente a u, então,

$$
\lim \inf _{n \rightarrow \infty} J\left(u_{n}\right) \geq J(u)
$$

2. Se $\mathbf{M}$ é não limitado, então $\left.J\right|_{\mathbf{M}}$ é coercivo, ou seja

$$
\lim _{|u| \rightarrow \infty} J(u)=\infty
$$

Então $J$ admite um ponto de minimo em $\mathbf{M}$.

\section{A.4 Um mínimo sobre fluxos gradientes e funcionais de energia}

Seja $\mathcal{H}$ um espaço de Hilbert. A derivada de Gâteaux de uma função ${ }^{1} \mathcal{E}: \mathcal{H} \rightarrow \mathbb{R}$ num ponto $u \in \mathcal{H}$ é a transformação linear contínua $d \mathcal{E}(u)$ tal que

$$
d \mathcal{E}(u)(v):=\lim _{t \rightarrow 0} \frac{1}{t}(\mathcal{E}(u+t v)-\mathcal{E}(u)) .
$$

Mais geralmente, pode-se definir a derivada de $\mathcal{E}$ no sentido de Fréchet, que segue analogia similar com a teoria de funções de varias variáveis em $\mathbb{R}^{n}$, estando a derivada de Fréchet para a derivada de Gâteaux como a derivada total está para a derivada direcional. Precisamente, $\mathcal{E}$ será diferenciável no sentido de Fréchet em $u$ se para qualquer $v \in \mathcal{H}$ tivermos

$$
\mathcal{E}(u+t v)-\mathcal{E}(u)-d \mathcal{E}(u)(t v)=o(\|t v\|),
$$

onde $\lim _{t \rightarrow 0} \frac{1}{t} o(\|t v\|)=0$.

Mostra-se (veja [AH10, Proposition A.3, p. 272]) que um funcional $\mathcal{E}$ diferenciável em um ponto $u \in \mathcal{H}$ no sentido de Gâteaux admite derivada no sentido de Fréchet se existir um aberto $U \ni u$ tal que o mapa $d \mathcal{E}: U \mapsto \mathcal{H}^{*}$ é uma aplicação contínua.

Formalmente, o gradiente de um funcional definido em um espaço de Hilbert é definido como:

Definição 42. Se $\mathcal{E}: \mathcal{H} \rightarrow \mathbb{R}$ é uma aplicação diferenciável no sentido de Fréchet em um espaço de Hilbert $\mathcal{H}$, chamamos de gradiente do funcional $\mathcal{E}$ a única aplicação linear $\nabla \mathcal{E}: \mathcal{H} \rightarrow \mathcal{H}$ definida por

$$
d \mathcal{E}(u)(v)=\langle\nabla \mathcal{E}(u), v\rangle,
$$

\footnotetext{
${ }^{1}$ Esta definição vale para aplicações gerais entre espaços de Banach.
} 
para cada $u \in \mathcal{H}$.

Um fluxo gradiente é a solução maximal $\varphi: I \rightarrow \mathcal{H}$ do problema

$$
\left\{\begin{array}{l}
\frac{d}{d t} \varphi(t)=-\nabla \mathcal{E}(\varphi(t)), \\
\varphi(0)=u \in \mathcal{H}
\end{array}\right.
$$

onde $I$ é um subconjunto aberto da reta.

Seja $\Omega$ um aberto limitado com fronteira regular em $\mathbb{R}^{n}$. Um exemplo clássico de fluxo gradiente é obtido a partir do funcional de Dirichlet

$$
\mathcal{E}(u):=\frac{1}{2} \int_{\Omega}|\nabla u|^{2} d x,
$$

onde $u \in H^{1}(\Omega)$.

Note que

$$
\begin{gathered}
\left.\frac{d}{d t}\right|_{t=0} \mathcal{E}(u+t v)=\left.\frac{1}{2} \frac{d}{d t}\right|_{t=0} \int_{\Omega}|\nabla u+t \nabla v|^{2} d x, \\
\left.\frac{d}{d t}\right|_{t=0} \mathcal{E}(u+t v)=\int_{\Omega}\langle\nabla u, \nabla v\rangle .
\end{gathered}
$$

Assumindo que $\left.u\right|_{\partial \Omega}=0$ podemos integrar por partes, obtendo

$$
\left.\frac{d}{d t}\right|_{t=0} \mathcal{E}(u+t v)=-\int_{\Omega}(\Delta u) v .
$$

Logo, o fluxo gradiente associado a esse funcional resulta na equação do calor:

$$
\frac{\partial}{\partial t} u=\Delta u
$$


APÊNDICE A 


\section{Referências Bibliográficas}

[AB15] M.M. Alexandrino e R.G. Bettiol. Lie Groups and Geometric Aspects of Isometric Actions. Springer International Publishing, 2015. 17, 19, 20, 21, 23, 25, 27, 28, 29, 30

[ACG19] Marcos M. Alexandrino, Leonardo F. Cavenaghi e Icaro Gonçalves. On mean curvature flow of singular riemannian foliations: Non compact cases, 2019. 37

[ACG20] Marcos M. Alexandrino, Leonardo F. Cavenaghi e Icaro Gonçalves. On mean curvature flow of singular riemannian foliations: Noncompact cases. Differential Geometry and its Applications, 72:101664, 2020. 35

[AH10] B. Andrews e C. Hopper. The Ricci Flow in Riemannian Geometry: A Complete Proof of the Differentiable 1/4-Pinching Sphere Theorem. Number No 2011 in Lecture Notes in Mathematics. Springer, 2010. 34, 107, 110

[AR16] Marcos M Alexandrino e Marco Radeschi. Mean curvature flow of singular riemannian foliations. The Journal of Geometric Analysis, 26(3):2204-2220, 2016. ix, xi, 35

[Aub98] T. Aubin. Some Nonlinear Problems in Riemannian Geometry. Springer Monographs in Mathematics. Springer Berlin Heidelberg, 1998. 102, 107

[BG] Maria Alice Bozola Grou. Fibrados com curvatura não negativa. 96, 97

[Bre72] G. Bredon. Introduction to Compact Transformation Groups. Academic press, 1972. $17,20,21,52$

[Bre93] G.E. Bredon. Topology and Geometry. Graduate texts in mathematics. Springer-Verlag, 1993. 17

[Bre10] S. Brendle. Ricci Flow and the Sphere Theorem. Graduate studies in mathematics. American Mathematical Society, 2010. 34

[BS] S. Brendle e R. Schoen. Curvature, sphere theorems, and the ricci flow. 1, 9

[Cao09] Huai-Dong Cao. Recent progress on ricci solitons, 2009. 42, 43

[CDR92] L. Chaves, A. Derdzinski e A. Rigas. A condition for positivity of curvature. Boletim da Sociedade Brasileira de Matemática, 23:153-165, 1992. ix, xi, 59

[CG72] C. Clemens e P. Griffiths. The intermediate jacobian of the cubic threefold. Annals of Mathematics, 95:281-356, 1972. 44

[Che73] J. Cheeger. Some examples of manifolds of nonnegative curvature. J. Diff. Geom., 8:623-628, 1973. 2, 10, 55

[CN15] D Crowley e J Nordström. New invariants of g2-structures. Geometry \& Topology, 19(5):2949-2992, 2015. 1, 9

[CS18a] Leonardo F. Cavenaghi e Llohann D. Sperança. A metric deformation on fiber bundles and applications. arXiv e-prints, página arXiv:1801.06576, Janeiro 2018. 59, 79 
[CS18b] Leonardo F Cavenaghi e Llohann D Sperança. On the geometry of some equivariantly related manifolds. International Mathematics Research Notices, página rny268, 2018. $1,9,47,50,92$

[CS18c] Leonardo F Cavenaghi e Llohann D Sperança. On the Geometry of Some Equivariantly Related Manifolds. International Mathematics Research Notices, 11 2018. rny268. 2, 10,59

[CS19] L.F. Cavenaghi e L.D. Sperança. Positive ricci curvature on fiber bundles with compact structure group, 2019. 1, 9

[CW17a] D. Crowley e D. J. Wraith. Intermediate curvatures and highly connected manifolds. arXiv preprint arXiv:1704.07057, 2017. 1, 9

[CW17b] D. Crowley e D. J. Wraith. Positive Ricci curvature on highly connected manifolds. Journal of Differential Geometry, 106(2):187-243, 2017. 1, 9

[Dan11] M.Y Dancer, A.S. \& Wang. On ricci solitons of cohomogeneity one. Annals of Global Analysis and Geometry, 39, 2011. 43, 44

[DD87] F. Dobarro e E. Lami Dozo. Scalar curvature and warped products of riemann manifolds. Transactions of the American Mathematical Society, 303(1):161-168, 1987. 7, 15, 98

[DMR04] C. Durán, A. Mendoza e A. Rigas. Blakers-Massey elements and exotic diffeomorphisms of $S^{6}$ and $S^{14}$ via geodesics. Transactions of the American Mathematical Society, páginas 5025-5043, 2004. 2, 10

[dS69a] R. de Sapio. Differential structures on a product of spheres: II. Annals of Mathematics, 89:305-313, 1969. 52

[dS69b] R. de Sapio. Manifolds homeomorphic to sphere bundles over spheres. Bulletin of the American Mathematical Society, 75(1):59-63, 1969. 52

[Dur01] C. Durán. Pointed wiedersehen metrics on exotic spheres and diffeomorphisms of s6. Geometriae Dedicata, 88(1-3):199-210, 2001. 2, 10, 46, 53

[EK62] J. Eells e N. Kuiper. An invariant of certain smooth manifolds. Annali Mat. Pura e Appl., 60:413-443, 2962. 97

[ES64] James Eells e J. H. Sampson. Harmonic mappings of riemannian manifolds. American Journal of Mathematics, 86(1):109-160, 1964. 34

[EYTK96] Paul E. Ehrlich, Jung Yoon-Tae e Seon-Bu Kim. Constant scalar curvatures on warped product manifolds. Tsukuba J. Math., 20(1):239-256, 06 1996. 7, 15, 98

[FdC13] F. Flaherty e M.P. do Carmo. Riemannian Geometry. Mathematics: Theory \& Applications. Birkhäuser Boston, 2013. 17

[FLGR16] Manuel Fernández-López e Eduardo García-Río. Positively curved shrinking ricci solitons are compact. Proceedings of the American Mathematical Society, 144:369-378, 2016. 43

[FY92] K. Fukaya e T. Yamaguchi. The fundamental groups of almost nonnegatively curved manifolds. Ann. of Math., 136:253-333, 1992. 79

[GÁG16] David González-Álvaro e Luis Guijarro. Soft restrictions on positively curved riemannian submersions. The Journal of Geometric Analysis, 26(2):1442-1452, 2016. 86 
[GÁR17] David González-Álvaro e Marco Radeschi. A note on the petersen-wilhelm conjecture. arXiv preprint arXiv:1706.00366, 2017. 86

[GKS20] S. Goette, M. Kerin e K. Shankar. Highly connected 7-manifolds and non-negative sectional curvature. Annals of Mathematics, 191(3):829-892, 2020. 1, 9

[GL80] Mikhael Gromov e H. Blaine Lawson. Spin and scalar curvature in the presence of a fundamental group. i. Annals of Mathematics, 111(2):209-230, 1980. 97

[GM74] D. Gromoll e W. Meyer. An exotic sphere with nonnegative sectional curvature. Annals of Mathematics, páginas 401-406, 1974. 1, 9, 46

[GVWZ06] Karsten Grove, Luigi Verdiani, Burkhard Wilking e Wolfgang Ziller. Non-negative curvature obstructions in cohomogeneity one and the kervaire spheres. Annali della Scuola Normale Superiore di Pisa-Classe di Scienze, 5(2):159-170, 2006. 1, 9

[GVZ11] K. Grove, L. Verdiani e W. Ziller. An exotic $\left\{\mathrm{T}_{-}\{1\} \backslash\right.$ mathbb $\left.\{\mathrm{S}\}^{\wedge} 4\right\}$ with positive curvature. Geometric and Functional Analysis, 21(3):499-524, 2011. 1, 9

[GW09] D. Gromoll e G. Walshap. Metric Foliations and Curvature. Birkhäuser Verlag, Basel, 2009. $17,20,25,30,31,32,63,87$

[GZ00] K. Grove e W. Ziller. Curvature and symmetry of milnor spheres. Annals of Mathematics, 152:331-367, 2000. 1, 9

[Ham82] Richard S Hamilton. Three-manifolds with positive ricci curvature. Journal of Differential Geometry, 17(2):255-306, 1982. 41

[Ham88] Richard S Hamilton. The ricci flow on surfaces. Mathematics and General Relativity, 71, 1988. 42

[Hit74] N. Hitchin. Harmonic spinors. Advances in Mathematics, 14(1):1-55, 1974. 1, 9

[Joy00] D.D. Joyce. Compact Manifolds with Special Holonomy. Oxford mathematical monographs. Oxford University Press, 2000. 107

[JW08] M. Joachim e D. J. Wraith. Exotic spheres and curvature. Bulletin of the Mathematical Society, 45:595-616, 2008. http://www.ams.org/journals/bull/2008-45-04/ S0273-0979-08-01213-5/. 1, 9

[KN63] S. Kobayashi e K. Nomizu. Foundations of differential geometry, volume I. Interscience Publishers, 1963. 17, 24

[KW75a] Jerry L. Kazdan e F. W. Warner. A direct approach to the determination of gaussian and scalar curvature functions. Inventiones mathematicae, (28):227-230, 1975. ix, xi, $6,14,96$

[KW75b] Jerry L. Kazdan e F. W. Warner. Existence and conformal deformation of metrics with prescribed gaussian and scalar curvatures. Annals of Mathematics, 101(2):317-331, 1975. ix, xi, 6, 14, 95

[KW75c] Jerry L. Kazdan e F. W. Warner. Scalar curvature and conformal deformation of riemannian structure. J. Differential Geom., 10(1):113-134, 1975. ix, xi, 6, 14, 95

[Lee] J. Lee. Introductino to Smooth Manifolds. 17

[LS74] H. B. Lawson e Yau S. Scalar curvature, non-abelian group actions, and the degree of symmetry of exotic spheres. Comm. Math. Helv., 49:232-244, 1974. 5, 13, 73 
[M̈̈7] M. Müter. Krummüngserhöhende deformationen mittels gruppenaktionen. Tese de Doutorado, Westfälischen Wilhelms-Universität Münster, 1987. 2, 10, 55, 57, 81

[MG09] R. M. Martins e L. Grama. The Ricci flow of left invariant metrics on full flag manifold $\mathrm{SU}(3) / \mathrm{T}$ from a dynamical systems point of view. ArXiv e-prints, Março 2009. 1, 9

[MW17] Ovidiu Munteanu e Jiaping Wang. Positively curved shrinking ricci solitons are compact. J. Differential Geom., 106:499-505, 2017. 43

[Nas79] J. Nash. Positive Ricci curvature on fibre bundles. Journal of Differential Geometry, 14(2):241-254, 1979. 1, 9, 79, 85

[NW07] Lei Ni e Nolan Wallach. On a classification of gradient shrinking solitons. Mathematical Research Letters, 15, 11 2007. 43

[Per02] G. Perelman. The entropy formula for the Ricci flow and its geometric applications, Novembro 2002. 42

[Per03] G. Perelman. Ricci flow with surgery on three-manifolds. ArXiv Mathematics e-prints, Março 2003. 1, 9

[Pet06a] P. Petersen. Riemannian Geometry. Graduate Texts in Mathematics. Springer New York, 2006. 17, 37

[Pet06b] P. Petersen. Riemannian Geometry. Springer, 2006. 70

[Poo75] WA Poor. Some exotic spheres with positive Ricci curvature. Mathematische Annalen, 216(3):245-252, 1975. 1, 9, 79, 85

[PT88] R.S. Palais e C. Terng. Critical Point Theory and Submanifold Geometry. Critical Point Theory and Submanifold Geometry. Richard S. Palais, 1988. 40

[PW10] Peter Petersen e William Wylie. On the classification of gradient ricci solitons. Geometry Es Topology, 14:2277-2300, 2010. 43

[PW14] Curtis Pro e Frederick Wilhelm. Riemannian submersions need not preserve positive ricci curvature. Proceedings of the American Mathematical Society, 142(7):2529-2535, 2014. $3,11,92$

[Rad] M. Radeschi. Lecture notes on singular riemannian foliations. https://www.marcoradeschi.com/s/SRF-Lecture-Notes.pdf. 17

[Ses04] N. Sesum. Limiting behaviour of the Ricci flow. Tese de Doutorado, Massachusetts Institute of Technology, 2004. 42

[Sic15] Gaetano Siciliano. Problemas elípticos semilineares: uma introdução ao método variacional. Sociedade Brasileira de Matemática, 2015. 109

[Spe10] LD Sperança. On explicit constructions of exotic spheres. arXiv preprint arXiv:1010.6039, 2010. 2, 10

[Spe16a] L Sperança. Pulling back the gromoll-meyer construction and models of exotic spheres. Proceedings of the American Mathematical Society, 144(7):3181-3196, 2016. 2, 10

[Spe16b] L Sperança. Pulling back the gromoll-meyer construction and models of exotic spheres. Proceedings of the American Mathematical Society, 144(7):3181-3196, 2016. 52, 53, 91

[Spe16c] L. Sperança. An intrinsic definition for nonnegatively curved connections, 2016. 59, 86, 87 
[SS96] L.L. Scott e J.P. Serre. Linear Representations of Finite Groups. Graduate Texts in Mathematics. Springer New York, 1996. 67

[SSW15] C. Searle, P. Solórzano e F. Wilhelm. Regularization via Cheeger Deformations. ArXiv e-prints, Fevereiro 2015. 85, 86

[ST09] Lorenz Schwachhöfer e Kristopher Tapp. Homogeneous metrics with nonnegative curvature. Journal of Geometric Analysis, 19(4):929-943, Oct 2009. 1, 9

[Sta16] M. Statha. Invariant metrics on homogeneous spaces with equivalent isotropy summands. ArXiv e-prints, Março 2016. 1, 9

[SW15] C. Searle e F. Wilhelm. How to lift positive Ricci curvature. Geometry \& Topology, 19(3):1409-1475, 2015. ix, xi, 1, 3, 5, 9, 11, 13, 61, 66

[Thu82] William P. Thurston. Three dimensional manifolds, kleinian groups and hyperbolic geometry. Bull. Amer. Math. Soc. (N.S.), 6(3):357-381, 05 1982. 1, 9

[Top06] P. Topping. Lectures on the Ricci Flow. Lecture note series. Cambridge University Press, 2006. 34, 41, 42

[Wal04] G. Walschap. Metric Structures in Differential Geometry. Graduate Texts in Mathematics. Springer New York, 2004. 75

[Wil01] F. Wilhelm. Exotic spheres with lots of positive curvatures. J. Geometric Anal., 11:161186, 2001. 1, 9

[Wra97] D. J. Wraith. Exotic spheres with positive Ricci curvature. J. Differential Geom., 45(3):638-649, 1997. 1, 9

[Wra07] D. J. Wraith. New connected sums with positive Ricci curvature. Annals of Global Analysis and Geometry, 32(4):343-360, 2007. 1, 9

[Zil] W. Ziller. http://www.math.upenn.edu/ wziller/research.html. 2, 10, 55

[Zil07] Wolfgang Ziller. Examples of riemannian manifolds with non-negative sectional curvature. Surveys in differential geometry, 11:63-102, 2007. 1, 9

[Zil14] W. Ziller. Riemannian manifolds with positive sectional curvature. Lecture Notes in Mathematics, 2110, 2014. 1, 9 